Supporting Information

\title{
Gold(I)-Catalyzed Reactions between 2-(1-Alkynyl)-2-alken-1-ones and Vinyldiazo Ketones for Divergent Synthesis of Non-symmetric Heteroaryl-substituted Triarylmethanes: $N$ - versus $C$-attack Paths
}

\author{
Rahul Dadabhau Kardile and Rai-Shung Liu*
}

Frontier Research Center of Matter Science and Technology, Department of Chemistry, National Tsing Hua Universi-ty, Hsinchu, Taiwan, ROC------------------------E-mail:rsliu@mx.nthu.edu.tw

\section{Content:}

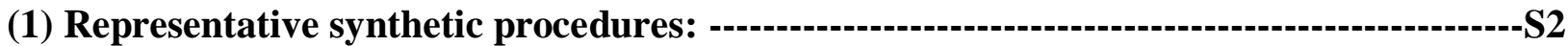

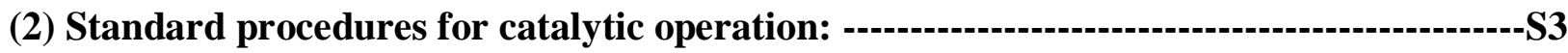

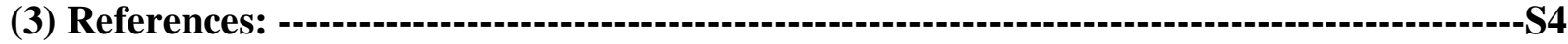

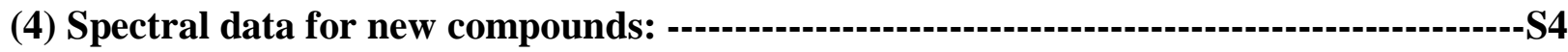

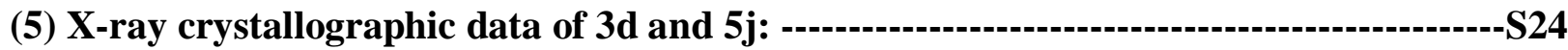

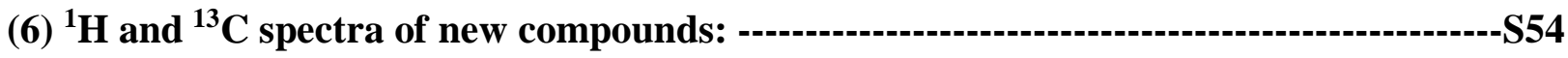




\section{Representative synthetic procedures:}

\section{(a) General procedure:}

Unless otherwise noted, all the reactions for the preparation of the substrates were performed in oven-dried glassware under nitrogen atmosphere with freshly distilled solvents. The catalytic reactions were performed under nitrogen atmosphere. DCM, DCE and toluene were distilled from $\mathrm{CaH}_{2}$ under nitrogen. THF was distilled from $\mathrm{Na}$ metal under nitrogen. All other commercial reagents were used without further purification, unless otherwise indicated. ${ }^{1} \mathrm{H} N M R$ and ${ }^{13} \mathrm{C}$ NMR spectra were recorded on a Varian $700 \mathrm{MHz}$, Bruker 400, $600 \mathrm{MHz}$ spectrometers using chloroform- $d\left(\mathrm{CDCl}_{3}\right)$ as the internal standard. High-resolution mass spectral analysis (HRMS) data were measured on JMS-T100LP4G (JEOL) mass spectrometer or a TOF mass analyzer equipped with the ESI source and Magnetic Sector Mass Analyzer (MStation) equipped with the EI source.

\section{(b) Preparation of enynones:}

All enynones (1a-1q) were prepared from procedure reported in the literature. ${ }^{[\mathrm{s} 1]}$

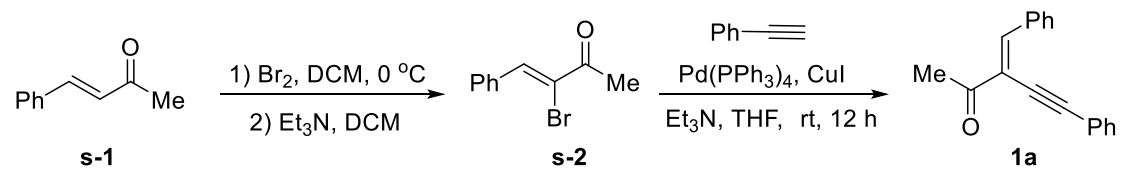

To a solution of benzalacetone (s-1) $(1.0 \mathrm{~g}, 6.84 \mathrm{mmol}, 1.0$ equiv.) in DCM (20 mL) was added $\mathrm{Br}_{2}(0.42 \mathrm{~mL}, 8.20 \mathrm{mmol}, 12$ equiv. $)$ at $0{ }^{\circ} \mathrm{C}$. The reaction mixture was stirred for $15 \mathrm{~min}$, followed by the addition of $\mathrm{Et}_{3} \mathrm{~N}$ (1.62 mL, $11.6 \mathrm{mmol}, 1.7$ equiv.). After stirring for additional $15 \mathrm{~min}$, the reaction mixture was diluted with DCM and washed sequentially with a $10 \% \mathrm{NaHSO}_{3}$ solution, $\mathrm{H}_{2} \mathrm{O}$ and brine. The organic layer was separated and dried over $\mathrm{MgSO}_{4}$, filtered and concentrated in vacuo. Crude product was purified on a silica gel column to give $\alpha$-bromobezalacetone (s-2) (1.38 g, $6.1 \mathrm{mmol}, 90 \%)$ as brown oil.

A round bottom flask was charged with $\mathrm{Pd}\left(\mathrm{PPh}_{3}\right)_{4}(17.6 \mathrm{mg}, 0.015 \mathrm{mmol}, 0.25 \mathrm{~mol} \%)$ and $\mathrm{CuI}$ (14.5 mg, $0.076 \mathrm{mmol}, 1.25 \mathrm{~mol} \%)$. To this was added freshly distilled THF (10 mL) and $\mathrm{Et}_{3} \mathrm{~N}$ $(10 \mathrm{~mL})$. The mixture was kept stirring and $\alpha$-bromobezalacetone (s-2) (1.38 g, $6.13 \mathrm{mmol}, 1$ equiv.) was added. Finally, phenylacetylene (0.75 g, $7.35 \mathrm{mmol}, 1.2$ equiv.) was added using a syringe over $30 \mathrm{~min}$. Then the mixture was stirred for $12 \mathrm{~h}$. Upon completion, the reaction mixture was quenched with aqueous $\mathrm{NH}_{4} \mathrm{Cl}$. Crude product was extracted with $\mathrm{Et}_{2} \mathrm{O}$ and concentrated in 
vacuo. The residue was purified on silica gel column to give pure enynone 1a $(1.44 \mathrm{~g}, 5.8 \mathrm{mmol}$, $96 \%)$.

(c) Preparation of vinyldiazo ketones:

All vinyldiazo ketones $(\mathbf{2 a - 2 m})$ were prepared from the reported procedure in the literatures. ${ }^{\text {[s2] }}$

\section{Standard procedures for catalytic operations:}

(a) Typical procedure for synthesis of 2-methyl-5-phenyl-3-(phenyl(5-phenylfuran-2yl)methyl)furan (3a):
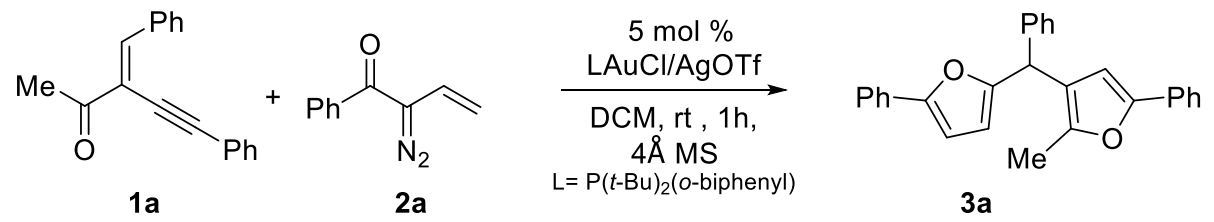

A suspension of $\mathrm{LAuCl}\left(\mathrm{L}=\mathrm{P}(t-\mathrm{Bu})_{2}(o\right.$-biphenyl) $(27 \mathrm{mg}, 0.05 \mathrm{mmol}), \operatorname{AgOTf}(13 \mathrm{mg}, 0.05$ $\mathrm{mmol})$ and $4 \AA \mathrm{AS}(40-50 \mathrm{mg})$ in dry DCM $(2 \mathrm{~mL})$ was fitted with $\mathrm{N}_{2}$ balloon and the mixture was stirred for $5 \mathrm{~min}$ at room temperature. To this solution was added a DCM (4 mL) solution of mixture of (E)-3-benzylidene-5-phenylpent-4-yn-2-one (1a) (250 mg, $1.0 \mathrm{mmol})$ and 2-diazo-1phenylbut-3-en-1-one (2a) (209 $\mathrm{mg}, 1.2 \mathrm{mmol})$ and the reaction was further stirred at room temperature. Upon completion, the reaction mixture was filtered over a short celite bed. The solvent was evaporated to dryness under reduced pressure, and the residue was purified on a silica gel column using ethyl acetate/hexane:01/99 as the eluent to give compound 3a (348 mg, 0.89 mmol, $88 \%$ ) yellow oil.

(b) Typical procedure for synthesis of (1-((2-methyl-5-phenylfuran-3-yl)(phenyl)methyl)$1 H$-pyrazol-3-yl)(phenyl)methanone (5a):

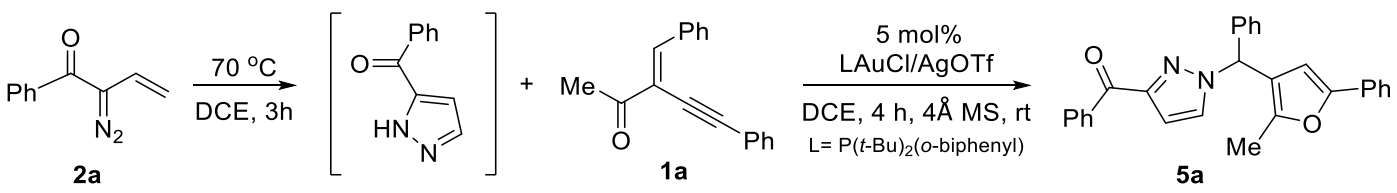

A DCE (2 mL) solution of 2-diazo-1-phenylbut-3-en-1-one (2a) (209 mg, $1.2 \mathrm{mmol})$ was heated at $70{ }^{\circ} \mathrm{C}$ (oil bath) for $3 \mathrm{~h}$ and then allowed to cool to room temperature. This cooled solution of 2a was added to $(E)-3$-benzylidene-5-phenylpent-4-yn-2-one (1a) (250 mg, $1.0 \mathrm{mmol})$ and the resulting solution was further added to a suspension of $\mathrm{LAuCl}\left(\mathrm{L}=\mathrm{P}(t-\mathrm{Bu})_{2}(o\right.$-biphenyl) $(27 \mathrm{mg}$, $0.05 \mathrm{mmol}), \operatorname{AgOTf}(13 \mathrm{mg}, 0.05 \mathrm{mmol})$ and $4 \AA \mathrm{MS}(40-50 \mathrm{mg})$ in dry DCE (4 mL) and the 
reaction was further stirred at room temperature. Upon completion, the reaction mixture was filtered over a short celite bed. The solvent was evaporated to dryness under reduced pressure, and the residue was purified on a silica gel column using ethyl acetate/hexane (10:90) as the eluent to give compound $\mathbf{5 a}(321 \mathrm{mg}, 0.82 \mathrm{mmol}, 81 \%)$ as yellow oil.

\section{References:}

[s1] Kardile, R. D.; Chao, T.-H.; Cheng, M.-J.; Liu, R.-S. Angew. Chem., Int. Ed. 2020, 59, $10396-10400$.

[s2] (a) Raj, A. S. K.; Liu, R.-S. Angew.Chem., Int. Ed. 2019, 58,10980-10984. (b) Raj, A. S. K.; Liu, R.-S. Adv. Synth. Catal. 2020, 362, 2517-2522. (c) Marichev, K. O.; Wang, Y.; Carranco, A. M.; Garcia, E. C.; Yu, Z.-X.; Doyle, M. P. Chem. Commun., 2018, 54, 95139516.

\section{Spectral data for new compounds:}

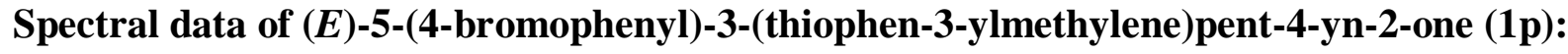

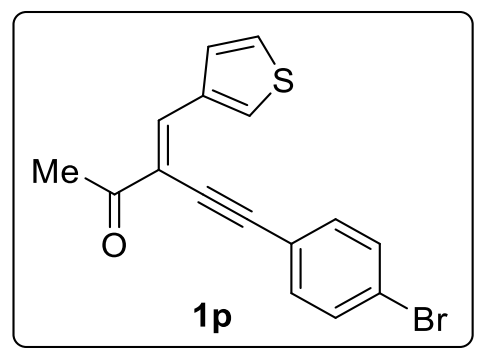

The residue was purified on silica gel column (ethyl acetate/hexane : 05/95) to give pure compound $1 p$ in $80 \%$ yield (Two steps); Brown oil ; ${ }^{1} \mathrm{H} \mathrm{NMR} \mathrm{(400} \mathrm{MHz,} \mathrm{CDCl}_{3}$ ): $\delta 8.08 \sim 8.07$ (m, 1H), $7.83(\mathrm{~s}, 1 \mathrm{H}), 7.78(\mathrm{~d}, \mathrm{~J}=5.2 \mathrm{~Hz}, 1 \mathrm{H}) 7.51(\mathrm{~d}, J=8.4 \mathrm{~Hz}, 2 \mathrm{H}), 7.39 \sim 7.34(\mathrm{~m}, 3 \mathrm{H}), 2.54(\mathrm{~s}, 3 \mathrm{H})$; ${ }^{13} \mathrm{C}$ NMR (100 MHz, $\left.\mathrm{CDCl}_{3}\right): \delta 195.9,137.0,136.9,132.7,131.9,131.2,128.4,126.1,123.3$, 121.7, 118.4, 97.9, 88.3, 28.1; HRMS (EI-MS) m/z: [M] ${ }^{+}$calcd. for $\mathrm{C}_{16} \mathrm{H}_{11} \mathrm{BrOS}[\mathrm{M}]^{+}$: 329.9714 ; found: 329.9715 
Spectral data of $(E)$-3-(4-nitrobenzylidene)-5-phenylpent-4-yn-2-one (1q):

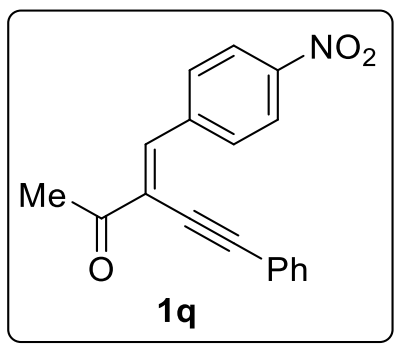

The residue was purified on silica gel column (ethyl acetate/hexane : 15/85) to give pure compound 1q in $73 \%$ yield (Two steps); Brown solid; m.p. 155.0 - $155.9{ }^{\circ} \mathrm{C} ;{ }^{1} \mathrm{H}$ NMR (400 MHz, $\mathrm{CDCl}_{3}$ ): $\delta$ $8.26(\mathrm{~d}, J=8.8 \mathrm{~Hz}, 2 \mathrm{H}), 8.19(\mathrm{~d}, J=8.8 \mathrm{~Hz}, 2 \mathrm{H}), 7.78(\mathrm{~s}, 1 \mathrm{H}), 7.54 \sim 7.52(\mathrm{~m}, 2 \mathrm{H}), 7.42 \sim 7.39$ (m, 3H), $2.63(\mathrm{~s}, 3 \mathrm{H}) ;{ }^{13} \mathrm{C}$ NMR $\left(125 \mathrm{MHz}, \mathrm{CDCl}_{3}\right): \delta 195.3,148.1,140.6,139.1,131.5,130.9$, 129.6, 128.7, 123.7, 123.3, 121.9, 100.9, 86.1, 28.1; HRMS (EI-MS) m/z: [M] ${ }^{+}$calcd. for $\mathrm{C}_{18} \mathrm{H}_{13} \mathrm{NO}_{3}[\mathrm{M}]^{+}: 291.0895$; found: 291.0891

Spectral data of 2-methyl-5-phenyl-3-(phenyl(5-phenylfuran-2-yl)methyl)furan (3a):

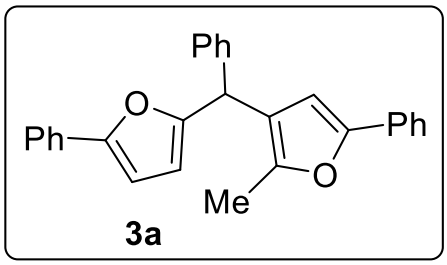

The residue was purified on silica gel column using ethyl acetate/hexane:01/99 as the eluent; Yellow oil (69 mg, $0.17 \mathrm{mmol}, 88 \%) ;{ }^{1} \mathrm{H}$ NMR (600 MHz, $\left.\mathrm{CDCl}_{3}\right): \delta 7.61 \sim 7.58(\mathrm{~m}, 4 \mathrm{H}), 7.34 \sim$ $7.18(\mathrm{~m}, 11 \mathrm{H}), 7.58(\mathrm{~d}, J=3.0 \mathrm{~Hz}, 1 \mathrm{H}), 6.47(\mathrm{~s}, 1 \mathrm{H}), 6.08(\mathrm{~d}, J=2.4 \mathrm{~Hz}, 1 \mathrm{H}), 5.32(\mathrm{~s}, 1 \mathrm{H}), 2.25$ (s, 3H); ${ }^{13} \mathrm{C}$ NMR (150 MHz, $\left.\mathrm{CDCl}_{3}\right): \delta 155.8,153.2,151.2,147.9,141.5,130.9,128.59,128.52$, 128.4, 128.2, 127.0, 126.77, 126.74, 123.5, 123.2, 121.7, 109.6, 107.0, 105.5, 41.8, 11.9, one carbon merged with other peak; HRMS (EI-MS) m/z: [M] calcd. for $\mathrm{C}_{28} \mathrm{H}_{22} \mathrm{O}_{2}: 390.1620$; found: 390.1623

Spectral data of 2-methyl-3-(phenyl(5-phenylfuran-2-yl)methyl)-5-(p-tolyl)furan (3b):

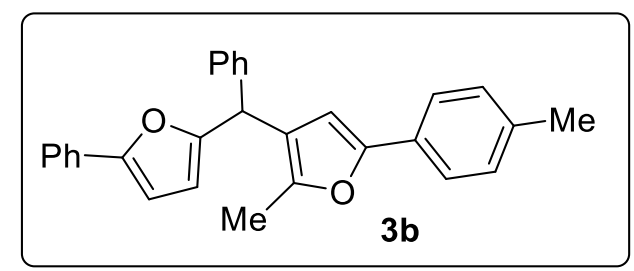


The residue was purified on silica gel column using ethyl acetate/hexane:01/99 as the eluent; Yellow oil (64 mg, $0.15 \mathrm{mmol}, 83 \%) ;{ }^{1} \mathrm{H}$ NMR (400 MHz, $\left.\mathrm{CDCl}_{3}\right): \delta 7.60 \sim 7.58(\mathrm{~m}, 2 \mathrm{H}), 7.46(\mathrm{~d}$, $J=8.4 \mathrm{~Hz}, 2 \mathrm{H}), 7.34 \sim 7.19(\mathrm{~m}, 8 \mathrm{H}), 7.11(\mathrm{~d}, J=8.4 \mathrm{~Hz}, 2 \mathrm{H}), 6.57(\mathrm{~d}, J=3.6 \mathrm{~Hz}, 1 \mathrm{H}), 6.39$ (s, $1 \mathrm{H}), 6.07(\mathrm{~d}, J=2.8 \mathrm{~Hz}, 1 \mathrm{H}), 5.30(\mathrm{~s}, 1 \mathrm{H}), 2.31(\mathrm{~s}, 3 \mathrm{H}), 2.23(\mathrm{~s}, 3 \mathrm{H}) ;{ }^{13} \mathrm{C} \mathrm{NMR}(125 \mathrm{MHz}$, $\left.\mathrm{CDCl}_{3}\right): \delta 156.0,153.3,151.6,147.5,141.7,136.6,131.1,129.3,128.7,128.6,128.4,128.3,127.1$, 126.8, 123.6, 123.3, 121.7, 109.8, 106.4, 105.7, 42.0, 21.3, 12.1; HRMS (EI-MS) m/z: [M] ${ }^{+}$calcd. for $\mathrm{C}_{29} \mathrm{H}_{24} \mathrm{O}_{2}$ : 404.1776; found: 404.1778

Spectral data of 5-(4-methoxyphenyl)-2-methyl-3-(phenyl(5-phenylfuran-2-yl)methyl)furan (3c):

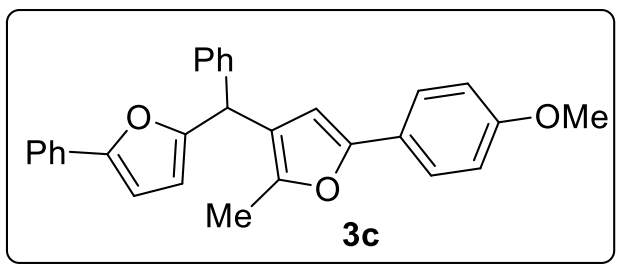

The residue was purified on silica gel column using ethyl acetate/hexane:01/99 as the eluent; Yellow oil (57 mg, $0.13 \mathrm{mmol}, 76 \%) ;{ }^{1} \mathrm{H}$ NMR (700 MHz, $\left.\mathrm{CDCl}_{3}\right): \delta 7.61(\mathrm{~d}, J=7.7 \mathrm{~Hz}, 2 \mathrm{H})$, $7.51(\mathrm{~d}, J=9.1 \mathrm{~Hz}, 2 \mathrm{H}), 7.34 \sim 7.19(\mathrm{~m}, 8 \mathrm{H}), 7.85(\mathrm{~d}, J=8.4 \mathrm{~Hz}, 2 \mathrm{H}), 6.58(\mathrm{~d}, J=3.5 \mathrm{~Hz}, 1 \mathrm{H})$, $6.33(\mathrm{~s}, 1 \mathrm{H}), 6.08(\mathrm{~d}, J=2.8 \mathrm{~Hz}, 1 \mathrm{H}), 5.30(\mathrm{~s}, 1 \mathrm{H}), 3.80(\mathrm{~s}, 3 \mathrm{H}), 2.24(\mathrm{~s}, 3 \mathrm{H}) ;{ }^{13} \mathrm{C}$ NMR $(175$ $\left.\mathrm{MHz}, \mathrm{CDCl}_{3}\right): \delta 158.6,155.9,153.1,151.3,147.1,141.6,130.9,128.5,128.4,128.2,127.0,126.6$, 124.7, 124.1, 123.5, 121.4, 113.9, 109.6, 105.5, 105.4, 55.2, 41.9, 11.9; HRMS (EI-MS) m/z: [M] ${ }^{+}$ calcd. for $\mathrm{C}_{29} \mathrm{H}_{24} \mathrm{O}_{3}$ : 420.1725; found: 420.1726

\section{Spectral data of 5-(4-bromophenyl)-2-methyl-3-(phenyl(5-phenylfuran-2-yl)methyl)furan} (3d):

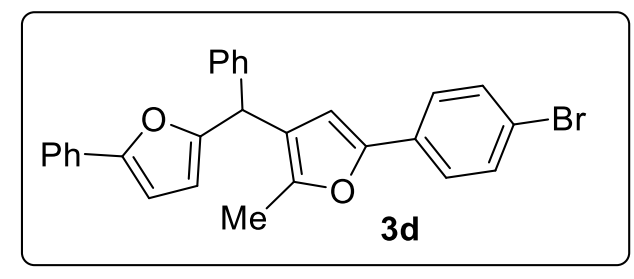

The residue was purified on silica gel column using ethyl acetate/hexane:01/99 as the eluent; yellow solid (56 mg, $0.11 \mathrm{mmol}, 78 \%$ ); m.p. 143.4 - $144.4{ }^{\circ} \mathrm{C} ;{ }^{1} \mathrm{H}$ NMR (400 $\left.\mathrm{MHz}, \mathrm{CDCl}_{3}\right): \delta$ $7.63 \sim 7.61(\mathrm{~m}, 3 \mathrm{H}), 7.46 \sim 7.22(\mathrm{~m}, 11 \mathrm{H}), 6.60(\mathrm{~d}, J=3.6 \mathrm{~Hz}, 1 \mathrm{H}), 6.48(\mathrm{~s}, 1 \mathrm{H}), 6.08(\mathrm{dd}, J=3.2$, $0.8 \mathrm{~Hz}, 1 \mathrm{H}), 5.33(\mathrm{~s}, 1 \mathrm{H}), 2.25(\mathrm{~s}, 3 \mathrm{H}) ;{ }^{13} \mathrm{C} \mathrm{NMR}\left(100 \mathrm{MHz}, \mathrm{CDCl}_{3}\right): \delta 155.7,153.3,150.3,148.4$, 
141.4, 131.6, 130.9, 129.8, 128.6, 128.5, 128.2, 127.1, 126.8, 124.8, 123.5, 122.0, 120.4, 109.7, 107.7, 105.6, 41.9, 12.0; HRMS (EI-MS) m/z: [M] calcd. for $\mathrm{C}_{28} \mathrm{H}_{21} \mathrm{BrO}_{2}$ : 468.0725; found: 468.0732

Spectral data of 5-(3-chlorophenyl)-2-methyl-3-(phenyl(5-phenylfuran-2-yl)methyl)furan (3e):

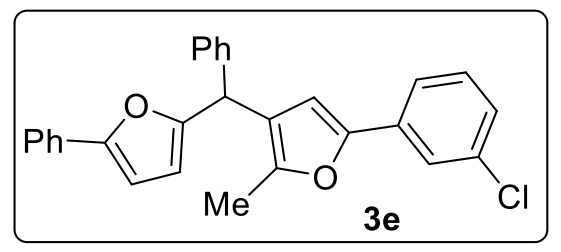

The residue was purified on silica gel column using ethyl acetate/hexane:01/99 as the eluent; Yellow oil (61 mg, $0.14 \mathrm{mmol}, 81 \%) ;{ }^{1} \mathrm{H}$ NMR (600 MHz, $\left.\mathrm{CDCl}_{3}\right): \delta 7.61 \sim 7.59(\mathrm{~m}, 2 \mathrm{H}), 7.56$ $(\mathrm{d}, J=1.8 \mathrm{~Hz}, 1 \mathrm{H}), 7.44(\mathrm{~d}, J=1.8 \mathrm{~Hz}, 1 \mathrm{H}), 7.34 \sim 7.30(\mathrm{~m}, 4 \mathrm{H}), 7.26 \sim 7.20(\mathrm{~m}, 5 \mathrm{H}), 7.14(\mathrm{~d}, J$ $=7.8 \mathrm{~Hz}, 1 \mathrm{H}), 6.58(\mathrm{~d}, J=3.0 \mathrm{~Hz}, 1 \mathrm{H}), 6.48(\mathrm{~s}, 1 \mathrm{H}), 6.07(\mathrm{~d}, J=3.0 \mathrm{~Hz}, 1 \mathrm{H}), 5.31(\mathrm{~s}, 1 \mathrm{H}), 2.25$ (s, 3H); ${ }^{13} \mathrm{C}$ NMR (150 MHz, $\left.\mathrm{CDCl}_{3}\right): \delta 155.6,153.3,149.8,148.6,141.3,134.5,132.5,130.8$, 129.8, 128.6, 128.5, 128.1, 127.1, 126.8, 126.6, 123.5, 123.2, 122.0, 121.3, 109.7, 108.1, 105.6, 41.8, 11.9; HRMS (EI-MS) m/z: [M] calcd. for $\mathrm{C}_{28} \mathrm{H}_{21} \mathrm{ClO}_{2}$ : 424.1230; found: 424.1236

Spectral data of 2-methyl-3-(phenyl(5-phenylfuran-2-yl)methyl)-5-(thiophen-3-yl)furan (3f):

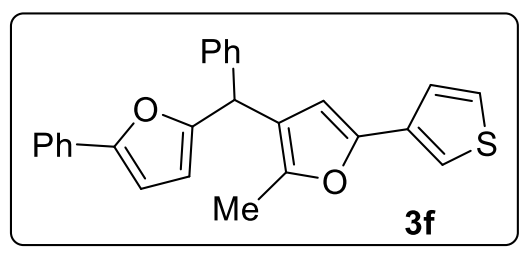

The residue was purified on silica gel column using ethyl acetate/hexane:01/99 as the eluent; Yellow oil (65 mg, $0.16 \mathrm{mmol}, 84 \%) ;{ }^{1} \mathrm{H}$ NMR (400 MHz, $\left.\mathrm{CDCl}_{3}\right): \delta 7.60 \sim 7.58(\mathrm{~m}, 2 \mathrm{H}), 7.35 \sim$ $7.19(\mathrm{~m}, 11 \mathrm{H}), 7.57(\mathrm{~d}, J=3.6 \mathrm{~Hz}, 1 \mathrm{H}), 6.27(\mathrm{~s}, 1 \mathrm{H}), 6.06(\mathrm{dd}, J=3.2,0.8 \mathrm{~Hz}, 1 \mathrm{H}), 5.29(\mathrm{~s}, 1 \mathrm{H})$, $2.22(\mathrm{~s}, 3 \mathrm{H}) ;{ }^{13} \mathrm{C}$ NMR $\left(150 \mathrm{MHz}, \mathrm{CDCl}_{3}\right): \delta 155.8,153.1,148.4,147.1,141.5,132.6,130.9$, 128.5, 128.4, 128.2, 127.0, 126.7, 125.9, 124.4, 123.5, 121.2, 117.8, 109.6, 106.7, 105.5, 41.8, 11.8; HRMS (EI-MS) m/z: [M] ${ }^{+}$calcd. for $\mathrm{C}_{26} \mathrm{H}_{20} \mathrm{O}_{2} \mathrm{~S}: 396.1184$; found: 396.1182

Spectral data of 5-cyclopropyl-2-methyl-3-(phenyl(5-phenylfuran-2-yl)methyl)furan (3g): 


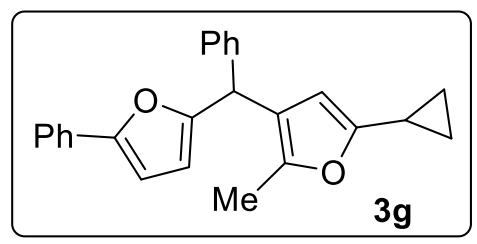

The residue was purified on silica gel column using ethyl acetate/hexane:01/99 as the eluent; Yellow oil (57 mg, $0.16 \mathrm{mmol}, 68 \%) ;{ }^{1} \mathrm{H}$ NMR (400 MHz, $\left.\mathrm{CDCl}_{3}\right): \delta 7.59(\mathrm{~d}, J=7.0 \mathrm{~Hz}, 2 \mathrm{H})$, $7.33 \sim 7.27(\mathrm{~m}, 4 \mathrm{H}), 7.22 \sim 7.19(\mathrm{~m}, 4 \mathrm{H}), 6.55(\mathrm{~d}, J=2.8 \mathrm{~Hz}, 1 \mathrm{H}), 6.03(\mathrm{~d}, J=2.8 \mathrm{~Hz}, 1 \mathrm{H}), 5.77$ (s, 1H), 5.20 (s, 1H), 2.12 (s, 3H), $1.79 \sim 1.75(\mathrm{~m}, 1 \mathrm{H}), 0.79 \sim 0.77(\mathrm{~m}, 2 \mathrm{H}), 0.70 \sim 0.67(\mathrm{~m}, 2 \mathrm{H})$; ${ }^{13} \mathrm{C}$ NMR $\left(175 \mathrm{MHz}, \mathrm{CDCl}_{3}\right): \delta 156.1,154.5,153.0,145.6,141.8,131.0,128.5,128.3,128.1$, 126.9, 126.5, 123.5, 119.7, 109.4, 105.5, 105.3, 41.9, 11.7, 8.7, 6.38, 6.35; HRMS (EI-MS) m/z: $[\mathrm{M}]^{+}$calcd. for $\mathrm{C}_{25} \mathrm{H}_{22} \mathrm{O}_{2}$ : 354.1620; found: 354.1621

Spectral data of 5-butyl-2-methyl-3-(phenyl(5-phenylfuran-2-yl)methyl)furan (3h):

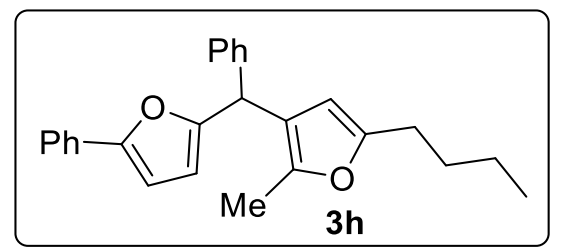

The residue was purified on silica gel column using ethyl acetate/hexane:01/99 as the eluent; Yellow oil (61 mg, $0.16 \mathrm{mmol}, 75 \%) ;{ }^{1} \mathrm{H}$ NMR (600 MHz, $\left.\mathrm{CDCl}_{3}\right): \delta 7.49 \sim 7.48(\mathrm{~m}, 2 \mathrm{H}), 7.23 \sim$ $7.17(\mathrm{~m}, 4 \mathrm{H}), 7.14 \sim 7.08(\mathrm{~m}, 4 \mathrm{H}), 6.46(\mathrm{~d}, J=3.6 \mathrm{~Hz}, 1 \mathrm{H}), 5.94 \sim 5.93(\mathrm{~m}, 1 \mathrm{H}), 5.69(\mathrm{~s}, 1 \mathrm{H}), 5.12$ (s, 1H), 2.40 (t, $J=7.8 \mathrm{~Hz}, 2 \mathrm{H}), 2.03(\mathrm{~s}, 3 \mathrm{H}), 1.48 \sim 1.43(\mathrm{~m}, 2 \mathrm{H}), 1.27 \sim 1.21(\mathrm{~m}, 2 \mathrm{H}), 0.79(\mathrm{t}, J$ $=7.8 \mathrm{~Hz}, 3 \mathrm{H}) ;{ }^{13} \mathrm{C} \mathrm{NMR}\left(150 \mathrm{MHz}, \mathrm{CDCl}_{3}\right): \delta 156.2,153.8,152.9,145.7,142.0,131.0,128.5$, 128.3, 128.1, 126.9, 126.5, 123.5, 119.6, 109.4, 106.4, 105.5, 41.9, 30.1, 27.7, 22.3, 13.8, 11.7; HRMS (EI-MS) m/z: [M] ${ }^{+}$calcd. for $\mathrm{C}_{26} \mathrm{H}_{26} \mathrm{O}_{2}: 370.1933$; found: 370.1930

\section{Spectral data of 2-methyl-5-phenyl-3-((5-phenylfuran-2-yl)(p-tolyl)methyl)furan (3i):}

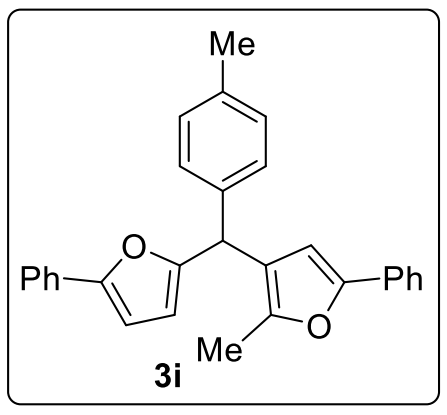


The residue was purified on silica gel column using ethyl acetate/hexane:01/99 as the eluent; Yellow oil (64 mg, $0.15 \mathrm{mmol}, 83 \%) ;{ }^{1} \mathrm{H}$ NMR (400 MHz, $\left.\mathrm{CDCl}_{3}\right): \delta 7.60 \sim 7.55(\mathrm{~m}, 4 \mathrm{H}), 7.33 \sim$ $7.28(\mathrm{~m}, 4 \mathrm{H}), 7.19 \sim 7.09(\mathrm{~m}, 6 \mathrm{H}), 6.56(\mathrm{~d}, J=3.2 \mathrm{~Hz}, 1 \mathrm{H}), 6.54(\mathrm{~s}, 1 \mathrm{H}), 6.06(\mathrm{dd}, J=4.0,0.8 \mathrm{~Hz}$, $1 \mathrm{H}), 5.26(\mathrm{~s}, 1 \mathrm{H}), 2.31(\mathrm{~s}, 3 \mathrm{H}), 2.24(\mathrm{~s}, 3 \mathrm{H}) ;{ }^{13} \mathrm{C} \mathrm{NMR}\left(150 \mathrm{MHz}, \mathrm{CDCl}_{3}\right): \delta 156.0,153.1,151.2$, $147.8,138.5,136.2,130.9,129.1,128.57,128.50,128.0,127.0,126.7,123.5,123.2,121.8,109.5$, 107.0, 105.5, 41.5, 21.0, 11.9, one carbon merged with other peak; HRMS (EI-MS) m/z: [M] ${ }^{+}$ calcd. for $\mathrm{C}_{29} \mathrm{H}_{24} \mathrm{O}_{2}$ : 404.1776; found: 404.1772

Spectral data of 3-((4-chlorophenyl)(5-phenylfuran-2-yl)methyl)-2-methyl-5-phenylfuran (3j):

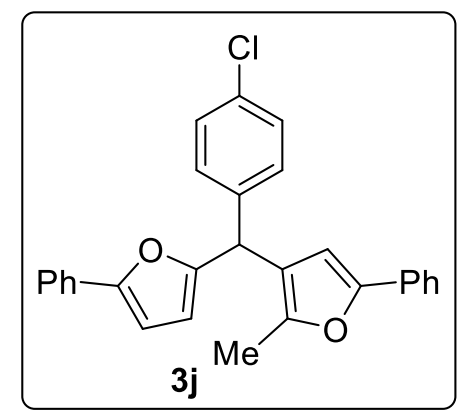

The residue was purified on silica gel column using ethyl acetate/hexane:01/99 as the eluent; Yellow oil (60 mg, $0.14 \mathrm{mmol}, 80 \%) ;{ }^{1} \mathrm{H}$ NMR (600 MHz, $\left.\mathrm{CDCl}_{3}\right): \delta 7.60 \sim 7.56(\mathrm{~m}, 4 \mathrm{H}), 7.35 \sim$ $7.24(\mathrm{~m}, 6 \mathrm{H}), 7.22 \sim 7.17(\mathrm{~m}, 4 \mathrm{H}), 6.57(\mathrm{~d}, J=3.6 \mathrm{~Hz}, 1 \mathrm{H}), 6.42(\mathrm{~s}, 1 \mathrm{H}), 6.07(\mathrm{dd}, J=3.6,1.2 \mathrm{~Hz}$, $1 \mathrm{H}), 5.28(\mathrm{~s}, 1 \mathrm{H}), 2.24(\mathrm{~s}, 3 \mathrm{H}) ;{ }^{13} \mathrm{C} \mathrm{NMR}\left(150 \mathrm{MHz}, \mathrm{CDCl}_{3}\right): \delta 155.1,153.4,151.4,147.9,140.0$, $132.5,130.79,130.74,129.5,128.6,128.5,127.1,126.9,123.5,123.2,121.2,109.8,106.7,105.5$, 41.3, 11.9 one carbon merged with other peak; HRMS (EI-MS) m/z: [M] calcd. for $\mathrm{C}_{28} \mathrm{H}_{21} \mathrm{ClO}_{2}$ : 424.1230; found: 424.1222

\section{Spectral data of 2-methyl-5-phenyl-3-((5-phenylfuran-2-yl)(thiophen-3-yl)methyl)furan} (3k):

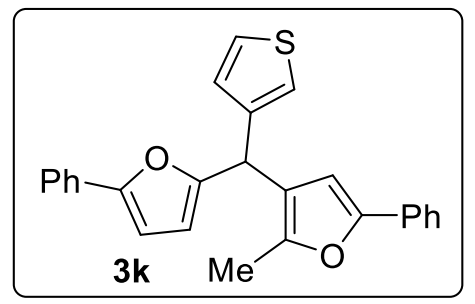

The residue was purified on silica gel column using ethyl acetate/hexane:01/99 as the eluent; Yellow oil (65 mg, 0.16 mmol, 83\%); ${ }^{1} \mathrm{H}$ NMR (400 MHz, $\left.\mathrm{CDCl}_{3}\right): \delta 7.61 \sim 7.56(\mathrm{~m}, 4 \mathrm{H}), 7.33 \sim$ 
$7.16(\mathrm{~m}, 7 \mathrm{H}), 7.01 \sim 7.00(\mathrm{~m}, 2 \mathrm{H}), 6.56(\mathrm{~d}, J=3.2 \mathrm{~Hz}, 1 \mathrm{H}), 6.50(\mathrm{~s}, 1 \mathrm{H}), 6.09(\mathrm{dd}, J=3.2,0.8 \mathrm{~Hz}$, 1H), $5.34(\mathrm{~s}, 1 \mathrm{H}), 2.26(\mathrm{~s}, 3 \mathrm{H}) ;{ }^{13} \mathrm{C} \mathrm{NMR}\left(150 \mathrm{MHz}, \mathrm{CDCl}_{3}\right): \delta$ 155.6, 153.1, 151.3, 147.8, 142.0, 130.9, 128.6, 128.5, 127.9, 127.0, 126.8, 125.6, 123.5, 123.2, 121.8, 121.4, 108.8, 106.8, 105.6, 37.5, 11.9, one carbon merged with other peak; HRMS (EI-MS) m/z: [M] ${ }^{+}$calcd. for $\mathrm{C}_{26} \mathrm{H}_{20} \mathrm{O}_{2} \mathrm{~S}$ : 396.1184; found: 396.1188

Spectral data of 2,5-diphenyl-3-(phenyl(5-phenylfuran-2-yl)methyl)furan (3I):

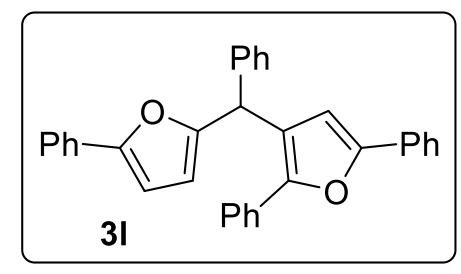

The residue was purified on silica gel column using ethyl acetate/hexane:01/99 as the eluent; Yellow oil (66 mg, $0.14 \mathrm{mmol}, 90 \%) ;{ }^{1} \mathrm{H}$ NMR (600 MHz, $\left.\mathrm{CDCl}_{3}\right): \delta 7.69 \sim 7.64(\mathrm{~m}, 2 \mathrm{H}), 7.62$ $(\mathrm{dd}, J=8.4,1.2 \mathrm{~Hz}), 7.42(\mathrm{t}, J=7.8 \mathrm{~Hz}, 2 \mathrm{H}), 7.38 \sim 7.20(\mathrm{~m}, 12 \mathrm{H}), 6.62 \sim 6.61(\mathrm{~m}, 2 \mathrm{H}), 6.15(\mathrm{dd}$, $J=3.6,1.2 \mathrm{~Hz}, 1 \mathrm{H}), 5.70(\mathrm{~s}, 1 \mathrm{H}) ;{ }^{13} \mathrm{C} \mathrm{NMR}\left(150 \mathrm{MHz}, \mathrm{CDCl}_{3}\right): \delta 155.7,153.4,152.3,148.7$, $141.3,130.88,130.84,130.5,128.69,128.60,128.3,127.5,127.3,127.1,126.9,126.2,123.7$, 123.5, 123.4, 110.1, 108.7, 105.6, 42.1, two carbons merged with other peak; HRMS (EI-MS) m/z: $[\mathrm{M}]^{+}$calcd. for $\mathrm{C}_{33} \mathrm{H}_{24} \mathrm{O}_{2}: 452.1776$; found: 152.1773

Spectral data of 2-butyl-5-phenyl-3-(phenyl(5-phenylfuran-2-yl)methyl)furan (3m):

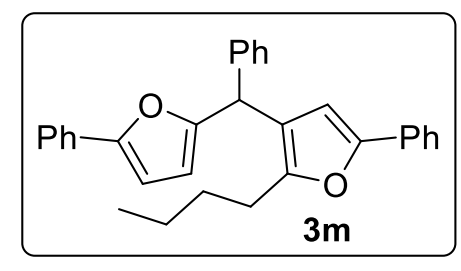

The residue was purified on silica gel column using ethyl acetate/hexane:01/99 as the eluent; Yellow oil (60 mg, $0.14 \mathrm{mmol}, 81 \%) ;{ }^{1} \mathrm{H}$ NMR (400 MHz, $\left.\mathrm{CDCl}_{3}\right): \delta 7.60 \sim 7.56$ (m, 4H), $7.34 \sim$ $7.15(\mathrm{~m}, 11 \mathrm{H}), 6.56(\mathrm{~d}, J=3.2 \mathrm{~Hz}, 1 \mathrm{H}), 6.45(\mathrm{~s}, 1 \mathrm{H}), 6.06(\mathrm{dd}, J=3.2,0.8 \mathrm{~Hz}, 1 \mathrm{H}), 5.32(\mathrm{~s}, 1 \mathrm{H})$, $2.59(\mathrm{t}, J=7.6 \mathrm{~Hz}, 2 \mathrm{H}), 1.63 \sim 1.54(\mathrm{~m}, 2 \mathrm{H}), 133 \sim 1.28(\mathrm{~m}, 2 \mathrm{H}), 0.85(\mathrm{t}, J=7.6 \mathrm{~Hz}, 3 \mathrm{H}) ;{ }^{13} \mathrm{C}$ NMR (150 MHz, $\left.\mathrm{CDCl}_{3}\right): \delta 156.0,153.1,152.0,151.2,141.7,131.0,130.9,128.57,128.51,128.4$, 128.2, 127.0, 126.75, 126.70, 123.5, 123.2, 121.4, 109.6, 106.8, 105.5, 41.7, 30.7, 26.0, 22.3, 13.8; HRMS (EI-MS) m/z: [M] ${ }^{+}$calcd. for $\mathrm{C}_{31} \mathrm{H}_{28} \mathrm{O}_{2}$ : 432.2089; found: 432.2086

Spectral data of 2-ethyl-5-phenyl-3-(phenyl(5-phenylfuran-2-yl)methyl)furan (3n): 


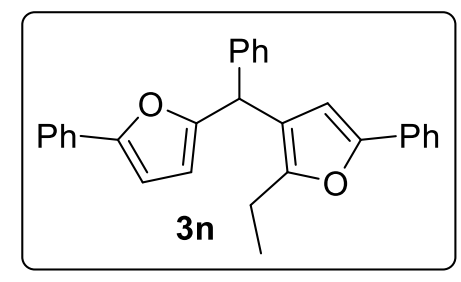

The residue was purified on silica gel column using ethyl acetate/hexane:01/99 as the eluent; Yellow oil (66 mg, $0.16 \mathrm{mmol}, 85 \%) ;{ }^{1} \mathrm{H}$ NMR (600 MHz, $\left.\mathrm{CDCl}_{3}\right): \delta 7.52 \sim 7.49(\mathrm{~m}, 4 \mathrm{H}), 7.25 \sim$ $7.07(\mathrm{~m}, 11 \mathrm{H}), 6.49(\mathrm{~d}, J=3.0 \mathrm{~Hz}, 1 \mathrm{H}), 6.38(\mathrm{~s}, 1 \mathrm{H}), 6.00(\mathrm{dd}, J=3.6,1.2 \mathrm{~Hz}, 1 \mathrm{H}), 5.26(\mathrm{~s}, 1 \mathrm{H})$, $2.54(\mathrm{q}, J=7.2 \mathrm{~Hz}, 2 \mathrm{H}), 1.12(\mathrm{t}, J=7.2 \mathrm{~Hz}, 3 \mathrm{H}) ;{ }^{13} \mathrm{C} \mathrm{NMR}\left(150 \mathrm{MHz}, \mathrm{CDCl}_{3}\right): \delta 156.0,153.2$, $152.9,151.3,141.7,131.0,130.9,128.6,128.56,128.51,128.2,127.0,126.8,126.7,123.5,123.2$, 120.9, 109.7, 106.9, 105.6, 41.7, 19.8, 13.1; HRMS (EI-MS) m/z: [M] $]^{+}$calcd. for $\mathrm{C}_{29} \mathrm{H}_{24} \mathrm{O}_{2}$ : 404.1776; found: 404.1772

Spectral data of 2-phenyl-4-(phenyl(5-phenylfuran-2-yl)methyl)furan (3o):

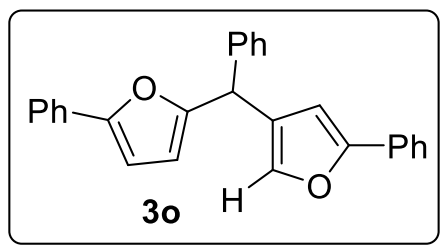

The residue was purified on silica gel column using ethyl acetate/hexane:01/99 as the eluent; Yellow oil (45 mg, $0.12 \mathrm{mmol}, 58 \%) ;{ }^{1} \mathrm{H}$ NMR (400 MHz, $\left.\mathrm{CDCl}_{3}\right): \delta 7.61 \sim 7.58(\mathrm{~m}, 4 \mathrm{H}), 7.35 \sim$ $7.30(\mathrm{~m}, 9 \mathrm{H}), 7.24 \sim 7.17(\mathrm{~m}, 3 \mathrm{H}), 6.57(\mathrm{~s}, 1 \mathrm{H}), 6.56(\mathrm{~s}, 1 \mathrm{H}), 6.10(\mathrm{dd}, J=2.8,0.8 \mathrm{~Hz}, 1 \mathrm{H}), 5.33$ $(\mathrm{s}, 1 \mathrm{H}) ;{ }^{13} \mathrm{C} \mathrm{NMR}\left(150 \mathrm{MHz}, \mathrm{CDCl}_{3}\right): \delta 155.7,154.2,153.2,141.0,139.9,130.9,130.7,128.6$, $128.5,128.3,127.3,127.1,126.9,123.7,123.5,109.4,106.3,105.6,42.4$, two carbons merged with other peak; HRMS (EI-MS) m/z: [M] ${ }^{+}$calcd. for $\mathrm{C}_{27} \mathrm{H}_{20} \mathrm{O}_{2}: 376.1463$; found: 376.1461

Spectral data of 2-methyl-3-((4-nitrophenyl)(5-phenylfuran-2-yl)methyl)-5-phenylfuran (3p):

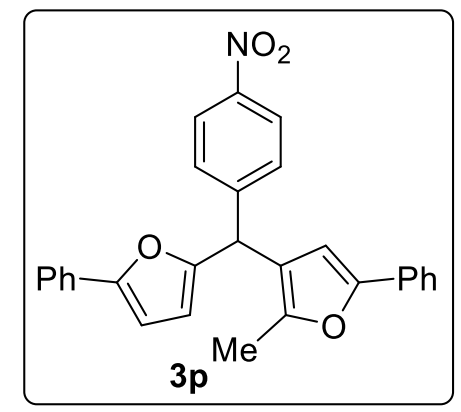


The residue was purified on silica gel column using ethyl acetate/hexane:03/97 as the eluent; Yellow oil (35 mg, $0.08 \mathrm{mmol}, 48 \%) ;{ }^{1} \mathrm{H}$ NMR (600 MHz, $\left.\mathrm{CDCl}_{3}\right): \delta 8.19(\mathrm{~d}, J=9.0 \mathrm{~Hz}, 2 \mathrm{H})$, $7.59 \sim 7.57(\mathrm{~m}, 4 \mathrm{H}), 7.42(\mathrm{~d}, J=9.0 \mathrm{~Hz}, 2 \mathrm{H}) 7.35 \sim 7.31(\mathrm{~m}, 4 \mathrm{H}), 7.24 \sim 7.19(\mathrm{~m}, 2 \mathrm{H}), 6.59(\mathrm{~d}, J$ $=3.6 \mathrm{~Hz}, 1 \mathrm{H}), 6.41(\mathrm{~s}, 1 \mathrm{H}), 6.14(\mathrm{dd}, J=3.6,1.2 \mathrm{~Hz}, 1 \mathrm{H}), 5.40(\mathrm{~s}, 1 \mathrm{H}) 2.27(\mathrm{~s}, 3 \mathrm{H}) ;{ }^{13} \mathrm{C} \mathrm{NMR}$ $\left(150 \mathrm{MHz}, \mathrm{CDCl}_{3}\right): \delta 153.8,151.9,149.0,148.1,146.9,130.59,130.56,129.0,128.69,128.61$, $127.4,127.1,123.8,123.5,123.3,120.3,110.2,106.3,105.6,41.7,11.9$, one carbon merged with other peak; HRMS (EI-MS) m/z: [M] ${ }^{+}$calcd. for $\mathrm{C}_{28} \mathrm{H}_{21} \mathrm{NO}_{4}$ : 435.1471; found: 435.1473

Spectral data of 2-methyl-5-phenyl-3-(phenyl(5-(p-tolyl)furan-2-yl)methyl)furan (4a):

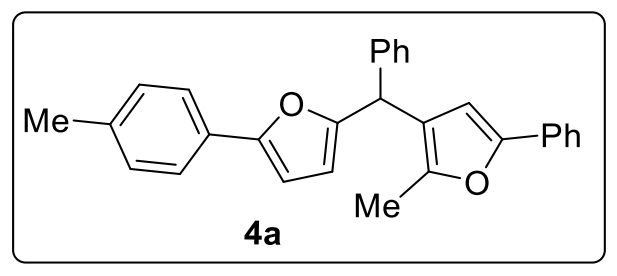

The residue was purified on silica gel column using ethyl acetate/hexane:01/99 as the eluent; Yellow oil (71 mg, $0.17 \mathrm{mmol}, 87 \%) ;{ }^{1} \mathrm{H}$ NMR (400 MHz, CDCl 3$): \delta 7.57(\mathrm{~d}, J=8.4 \mathrm{~Hz}, 2 \mathrm{H}$ ), $7.49(\mathrm{~d}, J=8.4 \mathrm{~Hz}, 2 \mathrm{H}), 7.32 \sim 7.23(\mathrm{~m}, 7 \mathrm{H}), 7.19 \sim 7.12(\mathrm{~m}, 3 \mathrm{H}), 7.21(\mathrm{~d}, J=3.2 \mathrm{~Hz}, 1 \mathrm{H}), 6.46$ (s, 1H), $6.06 \sim 6.04(\mathrm{~m}, 1 \mathrm{H}), 5.30(\mathrm{~s}, 1 \mathrm{H}), 2.32(\mathrm{~s}, 3 \mathrm{H}), 2.24(\mathrm{~s}, 3 \mathrm{H}) ;{ }^{13} \mathrm{C} \mathrm{NMR}\left(100 \mathrm{MHz}, \mathrm{CDCl}_{3}\right)$ : $\delta 155.4,153.5,151.3,147.9,141.6,136.9,130.9,129.3,128.57,128.52,128.2,126.8,126.7$, $123.5,123.3,121.8,109.6,107.1,104.8,41.9,21.2,12.0$, one carbon merged with other peak; HRMS (EI-MS) m/z: [M] ${ }^{+}$calcd. for $\mathrm{C}_{29} \mathrm{H}_{24} \mathrm{O}_{2}$ : 404.1776; found: 404.1769

\section{Spectral data of 3-((5-(4-methoxyphenyl)furan-2-yl)(phenyl)methyl)-2-methyl-5-} phenylfuran $(4 \mathrm{~b})$ :

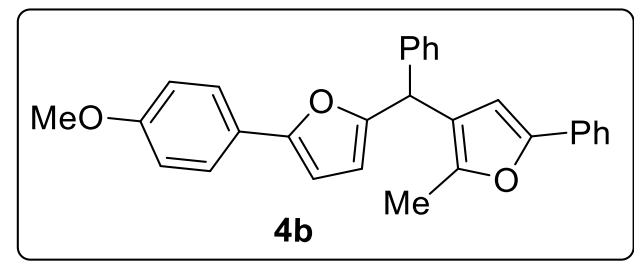

The residue was purified on silica gel column using ethyl acetate/hexane: 01/99 as the eluent; Yellow oil (68 mg, $0.16 \mathrm{mmol}, 80 \%) ;{ }^{1} \mathrm{H}$ NMR (600 MHz, $\left.\mathrm{CDCl}_{3}\right): \delta 7.60 \sim 7.58$ (m, 2H), 7.55 $(\mathrm{d}, J=9.0 \mathrm{~Hz}, 2 \mathrm{H}), 7.33 \sim 7.31(\mathrm{~m}, 4 \mathrm{H}), 7.29 \sim 7.27(\mathrm{~m}, 2 \mathrm{H}), 7.25 \sim 7.23(\mathrm{~m}, 1 \mathrm{H}), 7.20 \sim 7.17(\mathrm{~m}$ $1 \mathrm{H}), 6.88(\mathrm{~d}, J=9.0 \mathrm{~Hz}, 2 \mathrm{H}), 6.48(\mathrm{~s}, 1 \mathrm{H}), 6.45(\mathrm{~d}, J=3.6 \mathrm{~Hz}, 1 \mathrm{H}), 6.06(\mathrm{dd}, J=3.0,1.2 \mathrm{~Hz}, 1 \mathrm{H})$, $5.31(\mathrm{~s}, 1 \mathrm{H}), 3.80(\mathrm{~s}, 3 \mathrm{H}), 2.26(\mathrm{~s}, 3 \mathrm{H}) ;{ }^{13} \mathrm{C} \mathrm{NMR}\left(150 \mathrm{MHz}, \mathrm{CDCl}_{3}\right): \delta 158.8,155.0,153.2,151.2$, 
147.8, 141.6, 130.9, 128.5, 128.4, 128.2, 126.7, 126.6, 124.9, 124.0, 123.2, 121.8, 114.0, 109.5, 107.0, 103.9, 55.2, 41.8, 11.9; HRMS (EI-MS) m/z: [M] calcd. for $\mathrm{C}_{29} \mathrm{H}_{24} \mathrm{O}_{3}$ : 420.1725; found: 420.1722

Spectral data of 3-((5-(4-(tert-butyl)phenyl)furan-2-yl)(phenyl)methyl)-2-methyl-5phenylfuran $(4 \mathrm{c})$ :

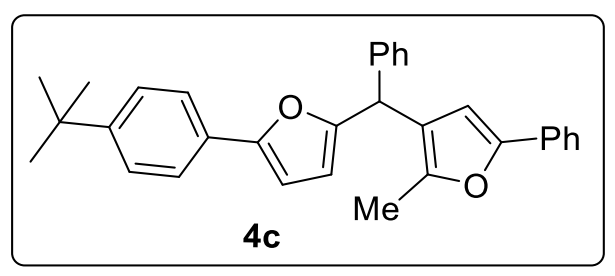

The residue was purified on silica gel column using ethyl acetate/hexane: 01/99 as the eluent; Yellow oil (74 mg, $0.16 \mathrm{mmol}, 82 \%) ;{ }^{1} \mathrm{H}$ NMR (600 MHz, $\left.\mathrm{CDCl}_{3}\right): \delta 7.60 \sim 7.58(\mathrm{~m}, 2 \mathrm{H}), 7.56$ $(\mathrm{d}, J=8.4 \mathrm{~Hz}, 2 \mathrm{H}), 7.37(\mathrm{~d}, J=8.4 \mathrm{~Hz}, 2 \mathrm{H}), 7.33 \sim 7.23(\mathrm{~m}, 7 \mathrm{H}), 7.20 \sim 7.17(\mathrm{~m}, 1 \mathrm{H}), 7.54(\mathrm{~d}, J$ $=3.0 \mathrm{~Hz}, 1 \mathrm{H}), 6.48(\mathrm{~s}, 1 \mathrm{H}), 6.07(\mathrm{dd}, J=3.6,1.2 \mathrm{~Hz}, 1 \mathrm{H}), 5.32(\mathrm{~s}, 1 \mathrm{H}), 2.26(\mathrm{~s}, 3 \mathrm{H}), 1.32(\mathrm{~s}, 9 \mathrm{H})$; ${ }^{13} \mathrm{C}$ NMR $\left(150 \mathrm{MHz}, \mathrm{CDCl}_{3}\right): \delta 155.4,153.4,151.2,150.1,147.9,141.6,130.9,128.5,128.4$, $128.3,128.2$, 126.78, 126.73, 125.5, 123.3, 123.2, 121.8, 109.6, 107.1, 105.0, 41.9, 34.5, 31.2, 12.0; HRMS (EI-MS) m/z: [M] ${ }^{+}$calcd. for $\mathrm{C}_{32} \mathrm{H}_{30} \mathrm{O}_{2}: 446.2246$; found: 446.2249

Spectral data of 3-((5-(4-chlorophenyl)furan-2-yl)(phenyl)methyl)-2-methyl-5-phenylfuran (4d):

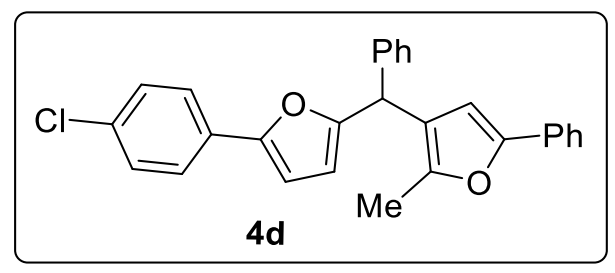

The residue was purified on silica gel column using ethyl acetate/hexane: 01/99 as the eluent; Yellow oil (64 mg, $0.15 \mathrm{mmol}, 75 \%) ;{ }^{1} \mathrm{H} \mathrm{NMR}\left(600 \mathrm{MHz}, \mathrm{CDCl}_{3}\right): \delta 7.60 \sim 7.58(\mathrm{~m}, 2 \mathrm{H}), 7.53 \sim$ $7.52(\mathrm{~m}, 2 \mathrm{H}), 7.34 \sim 7.23(\mathrm{~m}, 9 \mathrm{H}), 7.20 \sim 7.17(\mathrm{~m}, 1 \mathrm{H}), 6.57(\mathrm{~d}, J=3.0 \mathrm{~Hz}, 1 \mathrm{H}), 6.46(\mathrm{~s}, 1 \mathrm{H}), 6.09$ $(\mathrm{dd}, J=3.0,1.2 \mathrm{~Hz}, 1 \mathrm{H}), 5.32(\mathrm{~s}, 1 \mathrm{H}), 2.26(\mathrm{~s}, 3 \mathrm{H}) ;{ }^{13} \mathrm{C} \mathrm{NMR}\left(150 \mathrm{MHz}, \mathrm{CDCl}_{3}\right): \delta 156.2,152.1$, $151.3,147.8,141.3,132.6,130.8,129.3,128.7,128.5,128.1,126.8,124.7,123.2,121.5,109.8$, 106.9, 106.0, 41.8, 11.9, two carbons merged with other peak; HRMS (EI-MS) m/z: $[\mathrm{M}]^{+}$calcd. for $\mathrm{C}_{28} \mathrm{H}_{21} \mathrm{ClO}_{2}$ : 424.1230; found: 424.1237 
Spectral data of 3-((5-(4-bromophenyl)furan-2-yl)(phenyl)methyl)-2-methyl-5-phenylfuran (4e):

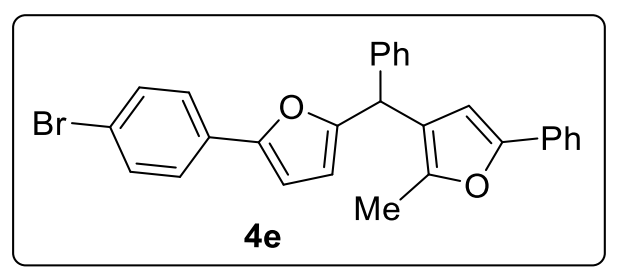

The residue was purified on silica gel column using ethyl acetate/hexane: 01/99 as the eluent; Yellow oil (75 mg, $0.16 \mathrm{mmol}, 79 \%)$; ${ }^{1} \mathrm{H}$ NMR (600 MHz, $\left.\mathrm{CDCl}_{3}\right): \delta 7.60 \sim 7.58$ (m, 2H), 7.46 $(\mathrm{d}, J=2.4 \mathrm{~Hz}, 4 \mathrm{H}), 7.34 \sim 7.31(\mathrm{~m}, 4 \mathrm{H}), 7.28 \sim 7.24(\mathrm{~m}, 3 \mathrm{H}), 7.21 \sim 7.18(\mathrm{~m}, 1 \mathrm{H}), 6.59(\mathrm{~d}, J=$ $3.6 \mathrm{~Hz}, 1 \mathrm{H}), 6.46(\mathrm{~s}, 1 \mathrm{H}), 6.09(\mathrm{dd}, J=3.0,1.2 \mathrm{~Hz}, 1 \mathrm{H}), 5.32(\mathrm{~s}, 1 \mathrm{H}), 2.26(\mathrm{~s}, 3 \mathrm{H}) ;{ }^{13} \mathrm{C} \mathrm{NMR}(150$ $\left.\mathrm{MHz}, \mathrm{CDCl}_{3}\right): \delta 156.3,152.1,151.3,147.9,141.3,131.7,130.8,129.8,128.5,128.1,126.8,125.2$, 123.2, 121.5, 120.7, 109.8, 106.9, 106.2, 41.9, 11.9, two carbons merged with other peak; HRMS (EI-MS) m/z: [M] $]^{+}$calcd. for $\mathrm{C} 28 \mathrm{H} 21 \mathrm{BrO}_{2}$ : 468.0725; found: 468.0728

\section{Spectral data of 2-methyl-5-phenyl-3-(phenyl(5-(thiophen-3-yl)furan-2-yl)methyl)furan} (4f):

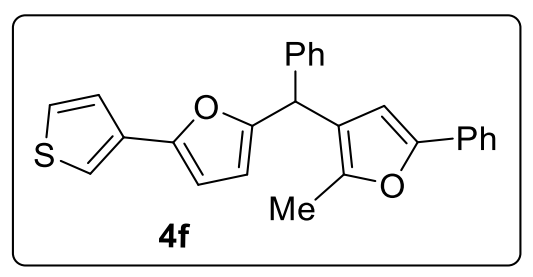

The residue was purified on silica gel column using ethyl acetate/hexane: 01/99 as the eluent; Yellow oil (69 mg, $0.17 \mathrm{mmol}, 84 \%)$; ${ }^{1} \mathrm{H}$ NMR (600 MHz, $\left.\mathrm{CDCl}_{3}\right): \delta 7.59 \sim 7.58(\mathrm{~m}, 2 \mathrm{H}), 7.38$ $(\mathrm{dd}, J=3.0,1.2 \mathrm{~Hz}, 1 \mathrm{H}), 7.33 \sim 7.29(\mathrm{~m}, 4 \mathrm{H}), 7.28 \sim 7.23(\mathrm{~m}, 5 \mathrm{H}), 7.18(\mathrm{t}, J=7.8 \mathrm{~Hz}, 1 \mathrm{H}), 6.46$ $(\mathrm{s}, 1 \mathrm{H}), 6.40(\mathrm{~d}, J=3.6 \mathrm{~Hz}, 1 \mathrm{H}), 6.04(\mathrm{dd}, J=3.0,1.2 \mathrm{~Hz}, 1 \mathrm{H}), 5.30(\mathrm{~s}, 1 \mathrm{H}), 2.26(\mathrm{~s}, 3 \mathrm{H}) ;{ }^{13} \mathrm{C}$ NMR (150 MHz, $\left.\mathrm{CDCl}_{3}\right): \delta 155.1,151.2,150.3,147.8,141.5,132.6,130.9,128.5,128.4,128.2$, 126.78, 126.73, 126.0, 124.5, 123.2, 121.7, 118.4, 109.3, 107.0, 105.3, 41.8, 11.9; HRMS (EI-MS) $\mathrm{m} / \mathrm{z}:[\mathrm{M}]^{+}$calcd. for $\mathrm{C}_{26} \mathrm{H}_{20} \mathrm{O}_{2} \mathrm{~S}: 396.1184$; found: 396.1182

Spectral data of 2-methyl-3-((5-(naphthalen-2-yl)furan-2-yl)(phenyl)methyl)-5-phenylfuran (4g): 


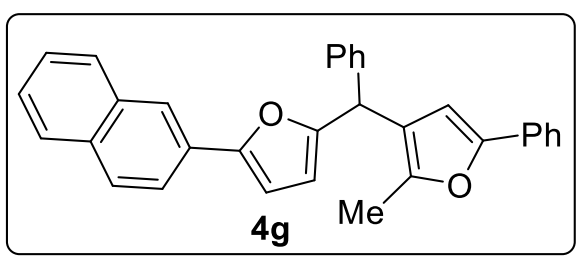

The residue was purified on silica gel column using ethyl acetate/hexane: 01/99 as the eluent; Yellow oil (72 mg, $0.16 \mathrm{mmol}, 81 \%) ;{ }^{1} \mathrm{H} \mathrm{NMR}\left(600 \mathrm{MHz}, \mathrm{CDCl}_{3}\right): \delta 8.09$ (s, 1H), $7.84 \sim 7.79(\mathrm{~m}$, 3H), $7.75 \sim 7.38(\mathrm{~m}, 1 \mathrm{H}), 7.64 \sim 7.62(\mathrm{~m}, 2 \mathrm{H}), 7.48 \sim 7.42(\mathrm{~m}, 2 \mathrm{H}), 7.38 \sim 7.33(\mathrm{~m}, 6 \mathrm{H}), 7.30 \sim$ $7.28(\mathrm{~s}, 1 \mathrm{H}), 7.23 \sim 7.20(\mathrm{~m}, 1 \mathrm{H}), 6.73(\mathrm{~d}, J=3.0 \mathrm{~Hz}, 1 \mathrm{H}), 6.54(\mathrm{~s}, 1 \mathrm{H}), 6.15(\mathrm{~d}, J=3.0 \mathrm{~Hz}, 1 \mathrm{H})$, $5.41(\mathrm{~s}, 1 \mathrm{H}), 2.30(\mathrm{~s}, 3 \mathrm{H}) ;{ }^{13} \mathrm{C} \mathrm{NMR}\left(150 \mathrm{MHz}, \mathrm{CDCl}_{3}\right): \delta 156.2,153.3,151.3,147.9,141.5,133.5$, $132.5,130.9,128.5,128.28,128.26,128.0,127.7,126.8,126.3,125.7,123.2,122.1,121.7,121.6$, 109.9, 107.0, 106.3, 41.9, 12.0, three carbons merged with other peak; HRMS (EI-MS) m/z: [M] ${ }^{+}$ calcd. for $\mathrm{C}_{32} \mathrm{H}_{24} \mathrm{O}_{2}$ : 440.1776; found: 440.1775

Spectral data of 3-((5-cyclopropylfuran-2-yl)(phenyl)methyl)-2-methyl-5-phenylfuran (4h):

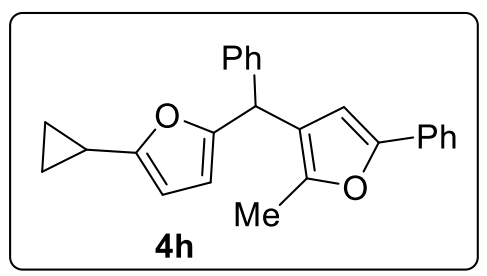

The residue was purified on silica gel column using ethyl acetate/hexane: 01/99 as the eluent; Yellow oil (50 mg, $0.14 \mathrm{mmol}, 70 \%) ;{ }^{1} \mathrm{H}$ NMR (400 MHz, $\left.\mathrm{CDCl}_{3}\right): \delta 7.56(\mathrm{~d}, J=8.0 \mathrm{~Hz}, 2 \mathrm{H})$, $7.32 \sim 7.26(\mathrm{~m}, 4 \mathrm{H}), 7.24 \sim 7.16(\mathrm{~m}, 4 \mathrm{H}), 6.39(\mathrm{~s}, 1 \mathrm{H}), 5.84 \sim 5.82(\mathrm{~m}, 2 \mathrm{H}), 5.16(\mathrm{~s}, 1 \mathrm{H}), 2.20(\mathrm{~s}$, $3 \mathrm{H}), 1.86 \sim 1.79(\mathrm{~m}, 1 \mathrm{H}), 0.82 \sim 0.78(\mathrm{~m}, 2 \mathrm{H}), 0.69 \sim 0.67(\mathrm{~m}, 2 \mathrm{H}) ;{ }^{13} \mathrm{C} \mathrm{NMR}\left(150 \mathrm{MHz}, \mathrm{CDCl}_{3}\right)$ : $\delta 156.8,153.8,151.1,147.7,141.8,131.0,128.5,128.3,128.1,126.7,126.5,123.2,121.9,108.0$, 107.1, 103.7, 41.7, 11.8, 8.8, 6.7, 6.6; HRMS (EI-MS) m/z: [M] ${ }^{+}$calcd. for $\mathrm{C}_{25} \mathrm{H}_{22} \mathrm{O}_{2}$ : 354.1620; found: 354.1618

Spectral data of 3-((5-butylfuran-2-yl)(phenyl)methyl)-2-methyl-5-phenylfuran (4i):

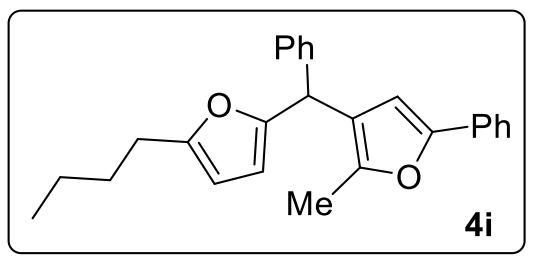


The residue was purified on silica gel column using ethyl acetate/hexane: 01/99 as the eluent; Yellow oil (54 mg, $0.14 \mathrm{mmol}, 72 \%) ;{ }^{1} \mathrm{H}$ NMR (600 MHz, $\left.\mathrm{CDCl}_{3}\right): \delta 7.59 \sim 7.58(\mathrm{~m}, 2 \mathrm{H}), 7.34 \sim$ $7.30(\mathrm{~m}, 4 \mathrm{H}), 7.25 \sim 7.22(\mathrm{~m}, 3 \mathrm{H}), 7.19(\mathrm{t}, J=7.8 \mathrm{~Hz}, 1 \mathrm{H}), 6.44(\mathrm{~s}, 1 \mathrm{H}), 5.90 \sim 5.87(\mathrm{~m}, 2 \mathrm{H}), 5.21$ (s, $1 \mathrm{H}), 2.59(\mathrm{t}, J=7.8 \mathrm{~Hz}, 2 \mathrm{H}), 2.24(\mathrm{~s}, 3 \mathrm{H}), 1.63 \sim 1.57(\mathrm{~m}, 2 \mathrm{H}), 1.39 \sim 1.33(\mathrm{~m}, 2 \mathrm{H}), 0.91(\mathrm{t}, J$ $=7.8 \mathrm{~Hz}, 3 \mathrm{H}) ;{ }^{13} \mathrm{C} \mathrm{NMR}\left(150 \mathrm{MHz}, \mathrm{CDCl}_{3}\right): \delta 155.9,154.0,151.1,147.7,141.8,131.0,128.5$, 128.3, 128.1, 126.6, 126.5, 123.2, 122.0, 107.9, 107.1, 105.0, 41.7, 30.2, 27.7, 22.2, 13.8, 11.8; HRMS (EI-MS) m/z: [M] $]^{+}$calcd. for $\mathrm{C}_{26} \mathrm{H}_{26} \mathrm{O}_{2}: 370.1933$; found: 370.1931

Spectral data of 2-methyl-3-((3-methyl-5-phenylfuran-2-yl)(phenyl)methyl)-5-phenylfuran $(4 \mathbf{j})$ :

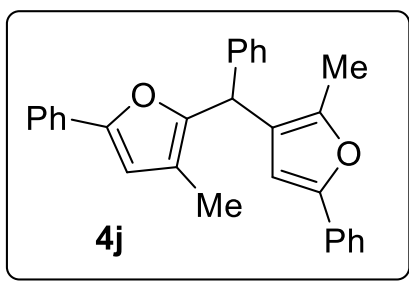

The residue was purified on silica gel column using ethyl acetate/hexane: 01/99 as the eluent; Yellow oil (55 mg, $0.13 \mathrm{mmol}, 67 \%)$; ${ }^{1} \mathrm{H} \mathrm{NMR}\left(600 \mathrm{MHz}, \mathrm{CDCl}_{3}\right): \delta 7.53(\mathrm{t}, J==7.2 \mathrm{~Hz}, 3 \mathrm{H})$, $7.33 \sim 7.26(\mathrm{~m}, 8 \mathrm{H}), 7.22 \sim 7.15(\mathrm{~m}, 4 \mathrm{H}), 6.57(\mathrm{~s}, 1 \mathrm{H}), 6.48(\mathrm{~s}, 1 \mathrm{H}), 5.30(\mathrm{~s}, 1 \mathrm{H}), 2.23(\mathrm{~s}, 3 \mathrm{H})$, $2.01(\mathrm{~s}, 3 \mathrm{H}) ;{ }^{13} \mathrm{C}$ NMR (150 MHz, $\left.\mathrm{CDCl}_{3}\right): \delta 151.7,151.1,150.0,147.8,141.6,131.0,128.59$, $128.52,128.4,128.1,127.4,126.8,126.7,126.5,123.4,123.2,121.6,117.3,108.5,107.5,39.4$, 11.9, 10.1; HRMS (EI-MS) m/z: [M] ${ }^{+}$calcd. for $\mathrm{C}_{29} \mathrm{H}_{24} \mathrm{O}_{2}$ : 404.1776; found: 404.1777

\section{Spectral data of 3-butyl-2-((2-methyl-5-phenylfuran-3-yl)(phenyl)methyl)-5-phenylfuran} (4k):

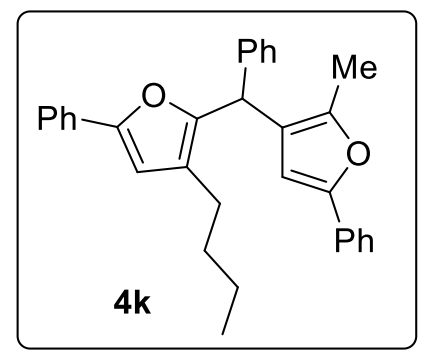

The residue was purified on silica gel column using ethyl acetate/hexane: 01/99 as the eluent; Yellow oil (57 mg, $0.12 \mathrm{mmol}, 63 \%) ;{ }^{1} \mathrm{H}$ NMR (600 MHz, $\left.\mathrm{CDCl}_{3}\right): \delta 7.59 \sim 7.57(\mathrm{~m}, 4 \mathrm{H}), 7.33 \sim$ $7.26(\mathrm{~m}, 8 \mathrm{H}), 7.22 \sim 7.16(\mathrm{~m}, 3 \mathrm{H}), 6.59(\mathrm{~s}, 1 \mathrm{H}), 6.53(\mathrm{~s}, 1 \mathrm{H}), 5.29(\mathrm{~s}, 1 \mathrm{H}), 2.41(\mathrm{t}, J=7.8 \mathrm{~Hz}, 2 \mathrm{H})$, $2.24(\mathrm{~s}, 3 \mathrm{H}), 1.54 \sim 1.49(\mathrm{~m}, 2 \mathrm{H}), 1.36 \sim 1.30(\mathrm{~m}, 2 \mathrm{H}), 0.88(\mathrm{t}, J=7.2 \mathrm{~Hz}, 3 \mathrm{H}) ;{ }^{13} \mathrm{C} \mathrm{NMR}(150$ 
$\left.\mathrm{MHz}, \mathrm{CDCl}_{3}\right): \delta 151.8,151.1,149.6,147.7,141.8,131.09,131.05,128.57,128.50,128.3,128.1$, 126.8, 126.6, 126.4, 123.3, 123.2, 122.6, 121.8, 107.6, 107.2, 39.3, 32.6, 24.5, 22.3, 13.9, 12.0; HRMS (EI-MS) m/z: [M] ${ }^{+}$calcd. for $\mathrm{C}_{32} \mathrm{H}_{30} \mathrm{O}_{2}$ : 446.2246; found: 446.2244

Spectral data of (1-((2-methyl-5-phenylfuran-3-yl)(phenyl)methyl)-1H-pyrazol-5yl)(phenyl)methanone (5a):

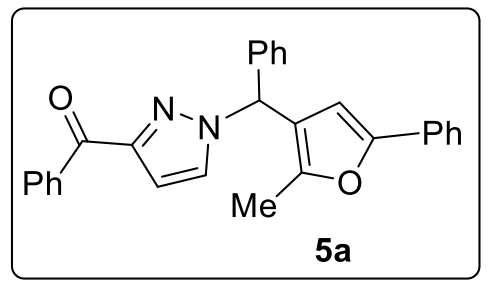

The residue was purified on silica gel column using ethyl acetate/hexane:10/90 as the eluent; Yellow oil (68 mg, $0.16 \mathrm{mmol}, 81 \%) ;{ }^{1} \mathrm{H}$ NMR (600 MHz, $\left.\mathrm{CDCl}_{3}\right): \delta 8.25 \sim 8.23(\mathrm{~m}, 2 \mathrm{H}), 7.59 \sim$ $7.58(\mathrm{~m}, 2 \mathrm{H}), 7.54 \sim 7.51(\mathrm{~m}, 2 \mathrm{H}), 7.42(\mathrm{t}, J=7.8 \mathrm{~Hz}, 2 \mathrm{H}), 7.36 \sim 7.31(\mathrm{~m}, 5 \mathrm{H}), 7.24 \sim 7.21(\mathrm{~m}$, $1 \mathrm{H}), 7.10(\mathrm{~d}, J=7.2 \mathrm{~Hz}, 2 \mathrm{H}), 7.00(\mathrm{~d}, J=2.4 \mathrm{~Hz}, 1 \mathrm{H}), 6.72(\mathrm{~s}, 1 \mathrm{H}), 6.42(\mathrm{~s}, 1 \mathrm{H}), 2.26(\mathrm{~s}, 3 \mathrm{H}) ;{ }^{13} \mathrm{C}$ NMR (150 MHz, $\left.\mathrm{CDCl}_{3}\right): \delta$ 187.8, 152.1, 150.7, 149.6, 139.3, 137.2, 132.5, 130.6, 130.3, 130.0, 128.7, 128.6, 128.09, 128.04, 127.3, 126.9, 123.4, 119.8, 109.3, 106.1, 62.5, 12.0; HRMS (ESITOF) m/z: [M+Na] $]^{+}$calcd. for $\mathrm{C}_{28} \mathrm{H}_{22} \mathrm{~N}_{2} \mathrm{O}_{2} \mathrm{Na}$ : 441.1579; found: 441.1581

Spectral data of (1-((2-methyl-5-(p-tolyl)furan-3-yl)(phenyl)methyl)-1H-pyrazol-5yl)(phenyl)methanone (5b):

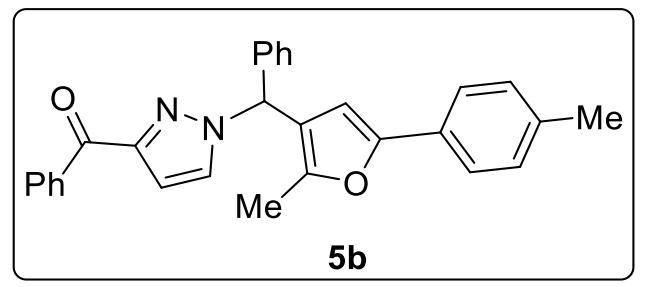

The residue was purified on silica gel column using ethyl acetate/hexane:10/90 as the eluent; Yellow oil (66 mg, $0.15 \mathrm{mmol}, 80 \%)$; ${ }^{1} \mathrm{H}$ NMR (600 MHz, $\left.\mathrm{CDCl}_{3}\right): \delta 8.24 \sim 8.22(\mathrm{~m}, 2 \mathrm{H}), 7.53 \sim$ $7.51(\mathrm{~m}, 2 \mathrm{H}), 7.47(\mathrm{~d}, J=8.4 \mathrm{~Hz}, 2 \mathrm{H}), 7.41(\mathrm{t}, J=7.8 \mathrm{~Hz}, 2 \mathrm{H}), 7.35 \sim 7.31(\mathrm{~m}, 3 \mathrm{H}), 7.14(\mathrm{~d}, J=$ $8.4 \mathrm{~Hz}, 2 \mathrm{H}), 7.09$ (d, J=7.2 Hz, 2H), $7.00(\mathrm{~d}, J=3.0 \mathrm{~Hz}, 1 \mathrm{H}), 6.71(\mathrm{~s}, 1 \mathrm{H}), 6.35(\mathrm{~s}, 1 \mathrm{H}), 2.33(\mathrm{~s}$, 3H), 2.24 (s, 3H); ${ }^{13} \mathrm{C}$ NMR (150 MHz, $\mathrm{CDCl}_{3}$ ): $\delta$ 187.8, 152.4, 150.7, 149.2, 139.3, 137.3, 137.2, 132.5, 130.6, 130.0, 129.3, 128.7, 128.0, 127.6, 126.9, 123.3, 119.6, 109.3, 105.3, 62.6, 21.2, 12.0, one carbon merged with other peaks; HRMS (ESI-TOF) m/z: [M+Na] ${ }^{+}$calcd. for $\mathrm{C}_{29} \mathrm{H}_{24} \mathrm{~N}_{2} \mathrm{O}_{2} \mathrm{Na}$ : 455.1735; found: 455.1736 
Spectral data of (1-((5-butyl-2-methylfuran-3-yl)(phenyl)methyl)-1H-pyrazol-5yl)(phenyl)methanone (5c):

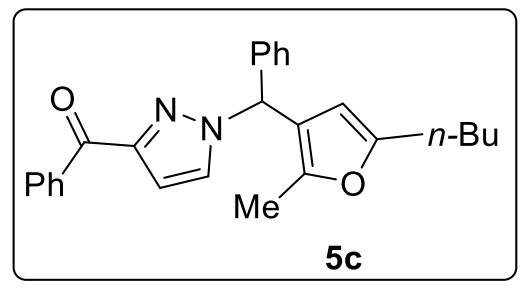

The residue was purified on silica gel column using ethyl acetate/hexane:10/90 as the eluent; Yellow oil (62 mg, $0.15 \mathrm{mmol}, 71 \%) ;{ }^{1} \mathrm{H}$ NMR (600 MHz, $\left.\mathrm{CDCl}_{3}\right): \delta 8.22 \sim 8.20(\mathrm{~m}, 2 \mathrm{H}), 7.53 \sim$ $7.50(\mathrm{~m}, 1 \mathrm{H}), 7.44 \sim 7.40(\mathrm{~m}, 3 \mathrm{H}), 7.33 \sim 7.27(\mathrm{~m}, 3 \mathrm{H}), 7.03 \sim 7.02(\mathrm{~m}, 2 \mathrm{H}), 6.96(\mathrm{~d}, J=2.4 \mathrm{~Hz}$, $1 \mathrm{H}), 6.63(\mathrm{~s}, 1 \mathrm{H}), 5.73(\mathrm{~s}, 1 \mathrm{H}), 2.51(\mathrm{t}, J=7.8 \mathrm{~Hz}, 2 \mathrm{H}), 2.12(\mathrm{~s}, 3 \mathrm{H}), 1.57 \sim 1.53(\mathrm{~m}, 2 \mathrm{H}), 1.36 \sim$ $1.30(\mathrm{~m}, 2 \mathrm{H}), 0.89(\mathrm{t}, J=7.8 \mathrm{~Hz}, 3 \mathrm{H}) ;{ }^{13} \mathrm{C} \mathrm{NMR}\left(150 \mathrm{MHz}, \mathrm{CDCl}_{3}\right): \delta 187.9,154.9,150.6,147.9$, 139.7, 137.3, 132.4, 130.6, 129.9, 128.6, 128.0, 127.8, 126.8, 117.7, 109.2, 105.7, 62.7, 30.0, 27.6, 22.2, 13.7, 11.8; HRMS (ESI-TOF) m/z: [M+Na] ${ }^{+}$calcd. for $\mathrm{C}_{26} \mathrm{H}_{26} \mathrm{~N}_{2} \mathrm{O}_{2} \mathrm{Na}$ : 421.1892; found: 421.1903

Spectral data of (1-((4-chlorophenyl)(2-methyl-5-phenylfuran-3-yl)methyl)-1H-pyrazol-5yl)(phenyl)methanone (5d):

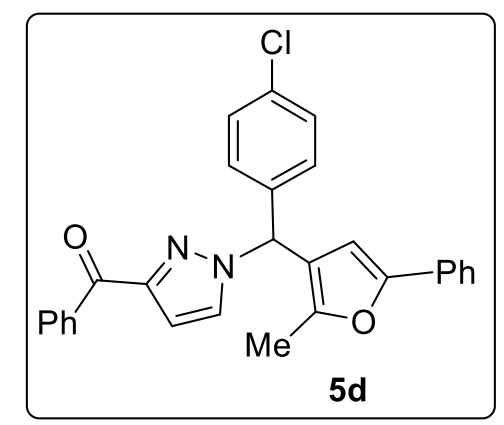

The residue was purified on silica gel column using ethyl acetate/hexane:10/90 as the eluent; Yellow oil (62 mg, $0.13 \mathrm{mmol}, 78 \%)$ ) ${ }^{1} \mathrm{H}$ NMR (600 MHz, $\left.\mathrm{CDCl}_{3}\right): \delta 8.23 \sim 8.21(\mathrm{~m}, 2 \mathrm{H}), 7.58$ $(\mathrm{d}, J=7.2 \mathrm{~Hz}, 2 \mathrm{H}), 7.54 \sim 7.50(\mathrm{~m}, 2 \mathrm{H}), 7.42(\mathrm{t}, J=7.2 \mathrm{~Hz}, 2 \mathrm{H}), 7.35 \sim 7.31(\mathrm{~m}, 4 \mathrm{H}), 7.24 \sim 7.21$ $(\mathrm{m}, 1 \mathrm{H}), 7.02 \sim 7.00(\mathrm{~m}, 3 \mathrm{H}), 6.68(\mathrm{~s}, 1 \mathrm{H}), 6.39(\mathrm{~s}, 1 \mathrm{H}), 2.27(\mathrm{~s}, 3 \mathrm{H}) ;{ }^{13} \mathrm{C} \mathrm{NMR}\left(150 \mathrm{MHz}, \mathrm{CDCl}_{3}\right)$ : $\delta 187.7,152.4,150.9,149.7,137.9,137.2,134.0,132.6,130.5,130.1,129.9,128.9,128.6,128.2$, 128.0, 127.4, 123.4, 119.3, 109.4, 105.8, 61.9, 12.0; HRMS (ESI-TOF) m/z: [M+Na] ${ }^{+}$calcd. for $\mathrm{C}_{28} \mathrm{H}_{21} \mathrm{ClN}_{2} \mathrm{O}_{2} \mathrm{Na}$ : 475.1189; found: 475.1200 
Spectral data of (4-methoxyphenyl)(1-((2-methyl-5-phenylfuran-3-yl)(phenyl)methyl)-1Hpyrazol-5-yl)methanone (5e):

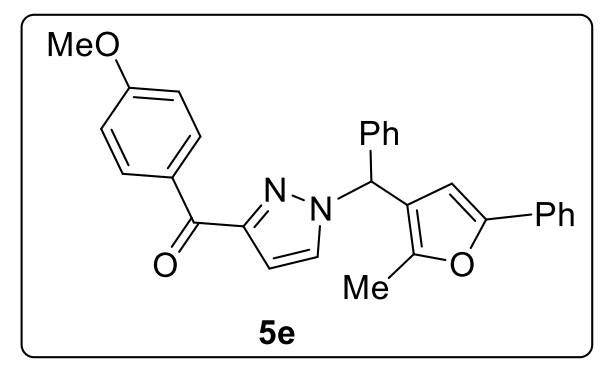

The residue was purified on silica gel column using ethyl acetate/hexane:15/85 as the eluent; Yellow oil (66 mg, $0.14 \mathrm{mmol}, 73 \%)$; ${ }^{1} \mathrm{H} \mathrm{NMR}\left(700 \mathrm{MHz}, \mathrm{CDCl}_{3}\right): \delta 8.25(\mathrm{~d}, J=8.4 \mathrm{~Hz}, 2 \mathrm{H})$, $7.52(\mathrm{~d}, J=8.4 \mathrm{~Hz}, 2 \mathrm{H}), 7.43(\mathrm{~d}, J=2.1 \mathrm{~Hz}, 1 \mathrm{H}), 7.29 \sim 7.24(\mathrm{~m}, 5 \mathrm{H}), 7.17 \sim 7.13(\mathrm{~m}, 1 \mathrm{H}), 7.03$ $(\mathrm{d}, J=7.0 \mathrm{~Hz}, 2 \mathrm{H}), 6.92(\mathrm{~d}, J=2.1 \mathrm{~Hz}, 1 \mathrm{H}), 6.83(\mathrm{~d}, J=9.1 \mathrm{~Hz}, 2 \mathrm{H}), 6.65(\mathrm{~s}, 1 \mathrm{H}), 6.36(\mathrm{~s}, 1 \mathrm{H})$, 3.75 (s, 3H), 2.19 (s, 3H); ${ }^{13} \mathrm{C}$ NMR (175 MHz, $\left.\mathrm{CDCl}_{3}\right): \delta 186.2,163.2,152.1,151.1,149.6,139.4$, 133.0, 130.3, 130.1, 129.9, 128.7, 128.6, 128.0, 127.3, 126.9, 123.4, 113.3, 109.2, 106.1, 62.4, 55.3, 12.0, one carbon merged with other peak; HRMS (ESI-TOF) $\mathrm{m} / \mathrm{z}$ : $[\mathrm{M}+\mathrm{Na}]^{+}$calcd. for $\mathrm{C}_{29} \mathrm{H}_{24} \mathrm{~N}_{2} \mathrm{O}_{3} \mathrm{Na}$ : 471.1685; found: 471.1677

Spectral data of (4-chlorophenyl)(1-((2-methyl-5-phenylfuran-3-yl)(phenyl)methyl)-1Hpyrazol-5-yl)methanone (5f):

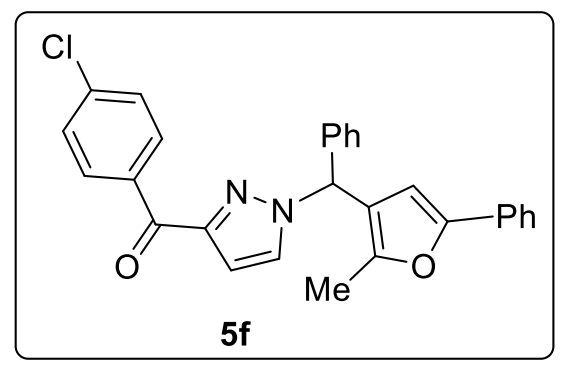

The residue was purified on silica gel column using ethyl acetate/hexane:10/90 as the eluent; Yellow oil (69 mg, $0.15 \mathrm{mmol}, 76 \%) ;{ }^{1} \mathrm{H} \mathrm{NMR}\left(700 \mathrm{MHz}, \mathrm{CDCl}_{3}\right): \delta 8.15(\mathrm{~d}, J=8.4 \mathrm{~Hz}, 2 \mathrm{H})$, $7.51(\mathrm{~d}, J=7.7 \mathrm{~Hz}, 2 \mathrm{H}), 7.45(\mathrm{~d}, J=2.1 \mathrm{~Hz}, 1 \mathrm{H}), 7.31(\mathrm{~d}, J=8.4 \mathrm{~Hz}, 2 \mathrm{H}), 7.28 \sim 7.25(\mathrm{~m}, 5 \mathrm{H})$, $7.17 \sim 7.14(\mathrm{~m}, 1 \mathrm{H}), 7.03(\mathrm{~d}, J=7.7 \mathrm{~Hz}, 2 \mathrm{H}), 6.94(\mathrm{~d}, J=2.1 \mathrm{~Hz}, 1 \mathrm{H}), 6.63(\mathrm{~s}, 1 \mathrm{H}), 6.34(\mathrm{~s}, 1 \mathrm{H})$, 2.19 (s, 3H); ${ }^{13} \mathrm{C}$ NMR $\left(175 \mathrm{MHz}, \mathrm{CDCl}_{3}\right): \delta 186.3,152.2,150.5,149.6,139.2,138.9,135.5$, 132.1, 130.27, 130.22, 128.8, 128.6, 128.3, 128.1, 127.3, 126.9, 123.4, 119.7, 109.3, 106.0, 62.6, 12.0; HRMS (ESI-TOF) m/z: [M+Na] ${ }^{+}$calcd. for $\mathrm{C}_{28} \mathrm{H}_{21} \mathrm{ClN}_{2} \mathrm{O}_{2} \mathrm{Na}$ : 475.1189 ; found: 475.1182 


\section{Spectral data of (1-((2-methyl-5-phenylfuran-3-yl)(phenyl)methyl)-1H-pyrazol-5-}

yl)(thiophen-3-yl)methanone (5g):

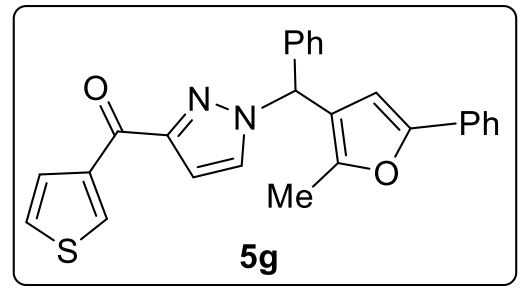

The residue was purified on silica gel column using ethyl acetate/hexane:10/90 as the eluent; Yellow oil (70 mg, $\left.0.16 \mathrm{mmol}, 82 \%) ;{ }^{1} \mathrm{H} \mathrm{NMR} \mathrm{(700} \mathrm{MHz,} \mathrm{CDCl}_{3}\right): \delta 8.73(\mathrm{~d}, J=2.8 \mathrm{~Hz}, 1 \mathrm{H})$, $7.84(\mathrm{~d}, J=4.9 \mathrm{~Hz}, 1 \mathrm{H}), 7.57(\mathrm{~d}, J=7.0 \mathrm{~Hz}, 2 \mathrm{H}), 7.48(\mathrm{~d}, J=2.1 \mathrm{~Hz}, 1 \mathrm{H}), 7.36 \sim 7.32(\mathrm{~m}, 5 \mathrm{H})$, $7.26 \sim 7.20(\mathrm{~m}, 2 \mathrm{H}), 7.11(\mathrm{~d}, J=7.7 \mathrm{~Hz}, 2 \mathrm{H}), 7.00(\mathrm{~d}, J=2.1 \mathrm{~Hz}, 1 \mathrm{H}), 6.69(\mathrm{~s}, 1 \mathrm{H}), 6.41(\mathrm{~s}, 1 \mathrm{H})$, $2.26(\mathrm{~s}, 3 \mathrm{H}) ;{ }^{13} \mathrm{C} \mathrm{NMR}\left(175 \mathrm{MHz}, \mathrm{CDCl}_{3}\right): \delta 180.6,152.2,151.3,149.6,140.7,139.3,135.4$, 130.3, 130.1, 128.8, 128.6, 128.1, 127.3, 126.9, 125.0, 123.4, 119.8, 108.8, 106.0, 62.6, 12.0, one carbon merged with other peak; HRMS (ESI-TOF) m/z: $[\mathrm{M}+\mathrm{Na}]^{+}$calcd. for $\mathrm{C}_{26} \mathrm{H}_{20} \mathrm{~N}_{2} \mathrm{O}_{2} \mathrm{SNa}$ : 447.1143; found: 447.1142

Spectral data of cyclopropyl(1-((2-methyl-5-phenylfuran-3-yl)(phenyl)methyl)-1H-pyrazol5-yl)methanone (5h):

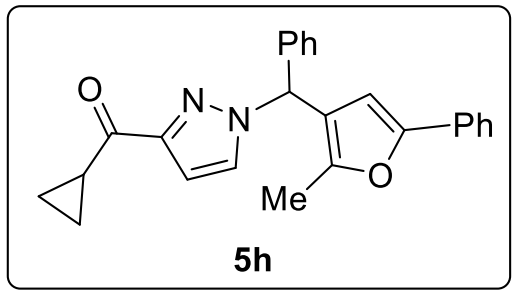

The residue was purified on silica gel column using ethyl acetate/hexane:10/90 as the eluent; Yellow oil (47 mg, $0.12 \mathrm{mmol}, 61 \%) ;{ }^{1} \mathrm{H} \mathrm{NMR}\left(700 \mathrm{MHz}, \mathrm{CDCl}_{3}\right): \delta 7.57(\mathrm{~d}, J=7.7 \mathrm{~Hz}, 2 \mathrm{H})$, $7.43(\mathrm{~d}, J=2.8 \mathrm{~Hz}, 1 \mathrm{H}), 7.35 \sim 7.31(\mathrm{~m}, 5 \mathrm{H}), 7.24 \sim 7.20(\mathrm{~m}, 1 \mathrm{H}), 7.08(\mathrm{~d}, J=7.7 \mathrm{~Hz}, 2 \mathrm{H}), 6.81$ $(\mathrm{d}, J=2.8 \mathrm{~Hz}, 1 \mathrm{H}), 6.69(\mathrm{~s}, 1 \mathrm{H}), 6.35(\mathrm{~s}, 1 \mathrm{H}), 3.02 \sim 2.98(\mathrm{~m}, 1 \mathrm{H}), 2.24(\mathrm{~s}, 3 \mathrm{H}), 1.24 \sim 1.19(\mathrm{~m}$, 2H), $0.98 \sim 0.96(\mathrm{~m}, 2 \mathrm{H}) ;{ }^{13} \mathrm{C} \mathrm{NMR}\left(175 \mathrm{MHz}, \mathrm{CDCl}_{3}\right): \delta 196.1,152.1,151.7,149.7,139.2,130.4$, 130.3, 128.7, 128.6, 128.1, 127.3, 126.9, 123.4, 119.7, 106.4, 106.0, 62.5, 17.2, 12.0, 11.7, 11.6; HRMS (ESI-TOF) m/z: [M+Na] ${ }^{+}$calcd. for $\mathrm{C}_{25} \mathrm{H}_{22} \mathrm{~N}_{2} \mathrm{O}_{2} \mathrm{Na}$ : 405.1579; found: 405.1571

Spectral data of (4-methyl-1-((2-methyl-5-phenylfuran-3-yl)(phenyl)methyl)-1H-pyrazol-5yl)(phenyl)methanone (5i): 


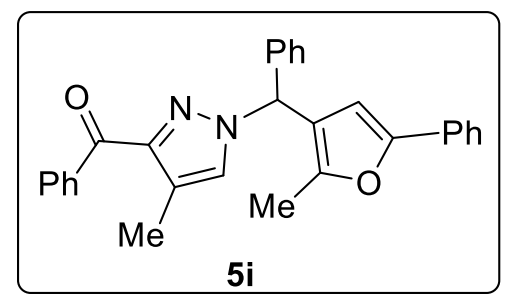

The residue was purified on silica gel column using ethyl acetate/hexane:10/90 as the eluent; Yellow oil (52 mg, $0.12 \mathrm{mmol}, 60 \%) ;{ }^{1} \mathrm{H}$ NMR (700 MHz, $\left.\mathrm{CDCl}_{3}\right): \delta 8.14(\mathrm{~d}, J=7.7 \mathrm{~Hz}, 2 \mathrm{H})$, $7.59(\mathrm{~d}, J=7.7 \mathrm{~Hz}, 1 \mathrm{H}), 7.39 \sim 7.29(\mathrm{~m}, 8 \mathrm{H}), 7.23 \sim 7.21(\mathrm{~m}, 1 \mathrm{H}), 7.08(\mathrm{~d}, 2 \mathrm{H}), 6.61(\mathrm{~s}, 1 \mathrm{H}), 6.41$ (s, 1H), $2.35(\mathrm{~s}, 3 \mathrm{H}), 2.25$ (s, 3H); ${ }^{13} \mathrm{C}$ NMR (175 MHz, $\left.\mathrm{CDCl}_{3}\right): \delta$ 189.4, 152.1, 149.6, 147.4, 139.4, 138.0, 132.2, 130.6, 130.3, 129.3, 128.7, 128.6, 128.0, 127.9, 127.3, 126.9, 123.4, 121.6, 119.9, 106.1, 62.3, 12.0, 10.2; HRMS (ESI-TOF) m/z: $[\mathrm{M}+\mathrm{Na}]^{+}$calcd. for $\mathrm{C}_{2} \mathrm{H}_{24} \mathrm{~N}_{2} \mathrm{O}_{2} \mathrm{Na}$ : 455.1735; found: 455.1732

Spectral data of (1-((5-(4-bromophenyl)-2-methylfuran-3-yl)(thiophen-3-yl)methyl)-1Hpyrazol-3-yl)(naphthalen-2-yl)methanone $(5 \mathbf{j})$ :

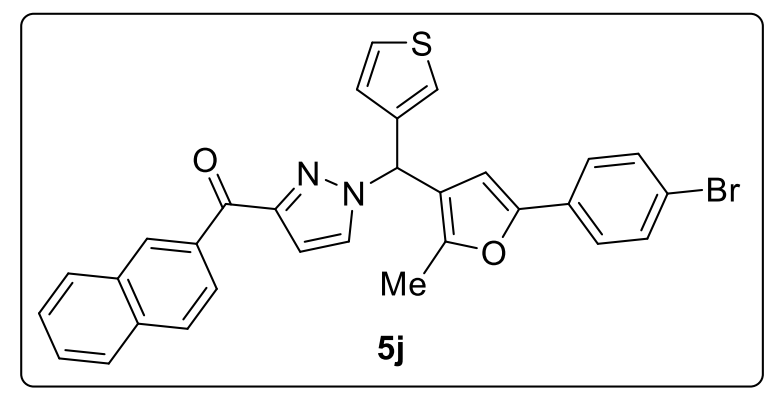

The residue was purified on silica gel column using ethyl acetate/hexane:10/90 as the eluent; Off white solid (69 mg, $0.12 \mathrm{mmol}, 83 \%$ ); m.p. 141.4 - 142.2 ${ }^{\circ} \mathrm{C} ;{ }^{1} \mathrm{H}$ NMR (400 MHz, $\mathrm{CDCl}_{3}$ ): $\delta 8.89$ (s, 1H), $8.23(\mathrm{dd}, J=8.4,1.6 \mathrm{~Hz}, 1 \mathrm{H}), 7.88 \sim 7.81(\mathrm{~m}, 3 \mathrm{H}), 7.57 \sim 7.53(\mathrm{~m}, 2 \mathrm{H}), 7.45(\mathrm{~s}, 5 \mathrm{H}), 7.35$ $7.33(\mathrm{~m}, 1 \mathrm{H}), 7.03(\mathrm{~d}, J=2.8 \mathrm{~Hz}, 2 \mathrm{H}), 7.95 \sim 7.94(\mathrm{~m}, 1 \mathrm{H}), 6.72(\mathrm{~s}, 1 \mathrm{H}), 6.56(\mathrm{~s}, 1 \mathrm{H}), 2.31(\mathrm{~s}$, $3 \mathrm{H}) ;{ }^{13} \mathrm{C} \mathrm{NMR}\left(100 \mathrm{MHz}, \mathrm{CDCl}_{3}\right): \delta 187.5,151.1,150.8,149.7,139.9,135.3,134.4,133.1,132.3$, $131.7,129.7,129.6,129.2,128.1,127.7,127.6,126.8,126.7,126.3,125.8,124.9,123.2,121.0$, 120.1, 109.4, 106.4, 58.8, 12.0; HRMS (ESI-TOF) m/z: $[\mathrm{M}+\mathrm{Na}]^{+}$calcd. for $\mathrm{C}_{30} \mathrm{H}_{21} \mathrm{BrN}_{2} \mathrm{O}_{2} \mathrm{SNa}$ : 575.0405; found: 575.0432 
Spectral data of ethyl 1-((2-methyl-5-phenylfuran-3-yl)(phenyl)methyl)-1H-pyrazole-3carboxylate (5k):

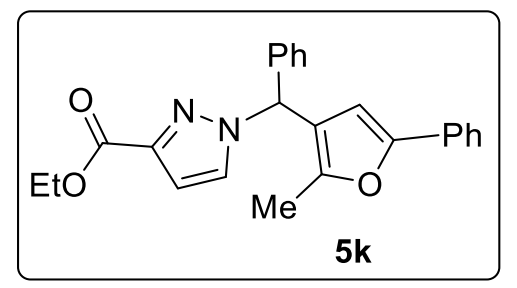

The residue was purified on silica gel column using ethyl acetate/hexane:15/85 as the eluent; Yellow oil (60 mg, $0.15 \mathrm{mmol}, 77 \%)$; ${ }^{1} \mathrm{H}$ NMR (400 MHz, $\left.\mathrm{CDCl}_{3}\right): \delta 7.55(\mathrm{~d}, J=7.6 \mathrm{~Hz}, 2 \mathrm{H})$, $7.42(\mathrm{~d}, J=2.4 \mathrm{~Hz}, 1 \mathrm{H}), 7.34 \sim 8.28(\mathrm{~m}, 6 \mathrm{H}), 7.20(\mathrm{t}, J=7.6 \mathrm{~Hz}, 1 \mathrm{H}), 7.05 \sim 7.03(\mathrm{~m}, 2 \mathrm{H}), 6.84$ (d, $J=2.4 \mathrm{~Hz}, 1 \mathrm{H}), 6.77(\mathrm{~s}, 1 \mathrm{H}), 6.29(\mathrm{~s}, 1 \mathrm{H}), 4.38(\mathrm{q}, J=7.2 \mathrm{~Hz}, 2 \mathrm{H}), 2.21(\mathrm{~s}, 3 \mathrm{H}), 1.37$ (t, $J=$ $7.2 \mathrm{~Hz}, 3 \mathrm{H}) ;{ }^{13} \mathrm{C}$ NMR (100 MHz, $\left.\mathrm{CDCl}_{3}\right): \delta 162.4,152.2,149.4,143.7,139.1,130.3,130.0$, 128.8, 128.6, 128.1, 127.3, 127.0, 123.4, 119.6, 109.0, 105.9, 62.7, 61.0, 14.2, 12.0; HRMS (ESITOF) m/z: [M+Na] ${ }^{+}$calcd. for $\mathrm{C}_{24} \mathrm{H}_{22} \mathrm{~N}_{2} \mathrm{O}_{3} \mathrm{Na}$ : 409.1528; found: 409.1535

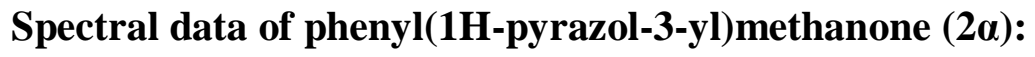

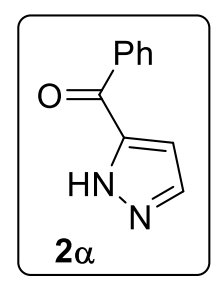

Dichloroethane (DCE, $2 \mathrm{~mL}$ ) solution of vinyldiazo ketone $2 \mathbf{a}(50 \mathrm{mg}, 0.29 \mathrm{mmol})$ was stirred at $70{ }^{\circ} \mathrm{C}$ (oil bath) for $3 \mathrm{~h}$. After the completion, the solvent was evaporated in vacuo under reduced pressure. The residue was purified on silica gel column using ethyl acetate/hexane:50/50 as the eluent to give pure compound $2 \boldsymbol{\alpha}$ as Yellow oil (46 mg, $0.27 \mathrm{mmol}, 93 \%) ;{ }^{1} \mathrm{H}$ NMR (600 MHz, $\left.\mathrm{CDCl}_{3}\right): \delta 8.05(\mathrm{~d}, J=7.8 \mathrm{~Hz}, 2 \mathrm{H}), 7.59(\mathrm{~d}, J=1.8 \mathrm{~Hz}, 2 \mathrm{H}), 7.49(\mathrm{t}, J=7.8,2 \mathrm{H}), 6.85(\mathrm{~d}, J=1.8$ $\mathrm{Hz}, 1 \mathrm{H}) ;{ }^{13} \mathrm{C}$ NMR $\left(150 \mathrm{MHz}, \mathrm{CDCl}_{3}\right): \delta 187.3,137.5,133.7,132.8,129.7,128.4$, 109.4, one carbon merged with another peak; ${ }^{1} \mathrm{H}$ NMR $\left(400 \mathrm{MHz}, \mathrm{CD}_{3} \mathrm{CN}\right): \delta 11.60(\mathrm{~s}, 1 \mathrm{H}), 8.13(\mathrm{~d}, J=7.6$ $\mathrm{Hz}, 2 \mathrm{H}), 7.70(\mathrm{~d}, J=2.4 \mathrm{~Hz}, 1 \mathrm{H}), 7.64 \sim 7.60(\mathrm{~m}, 1 \mathrm{H}), 7.51(\mathrm{t}, J=7.2 \mathrm{~Hz}, 2 \mathrm{H}), 6.89(\mathrm{~d}, J=2.4$ $\mathrm{Hz}, 1 \mathrm{H}$ ); HRMS (EI-MS) m/z: [M] calcd. for $\mathrm{C}_{10} \mathrm{H}_{8} \mathrm{~N}_{2} \mathrm{O}: 172.0637$; found: 172.0639 
Spectral data of 2-diazo-5-(2-methyl-5-phenylfuran-3-yl)-1,5-diphenylpentane-1,3-dione (6):

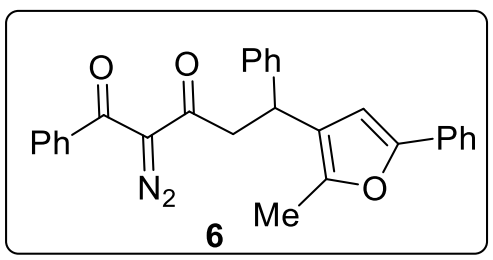

Following the standard procedure for catalytic operation, compound $\mathbf{6}$ was isolated in $70 \%$ yield using ethyl acetate/hexane:05/95 as the eluent for silica column; Yellow oil (61 mg, 0.14 mmol, $70 \%) ;{ }^{1} \mathrm{H}$ NMR (600 MHz, $\left.\mathrm{CDCl}_{3}\right): \delta 7.56 \sim 7.42(\mathrm{~m}, 7 \mathrm{H}), 7.34 \sim 7.17(\mathrm{~m}, 8 \mathrm{H}), 6.60(\mathrm{~s}, 1 \mathrm{H}), 4.57$ $(\mathrm{t}, 1 \mathrm{H}), 3.79 \sim 3.73(\mathrm{~m}, 1 \mathrm{H}), 3.63 \sim 3.57(\mathrm{~m}, 1 \mathrm{H}), 2.32(\mathrm{~s}, 3 \mathrm{H}) ;{ }^{13} \mathrm{C} \mathrm{NMR}\left(150 \mathrm{MHz}, \mathrm{CDCl}_{3}\right): \delta$ 191.6, 185.1, 151.4, 147.6, 143.5, 137.2, 132.7, 130.9, 128.9, 128.6, 128.5, 127.5, 127.3, 126.7, 126.5, 123.2, 105.6, 83.6, 46.5, 37.4, 11.9, one carbon merged with other peak; HRMS (ESI-TOF) $\mathrm{m} / \mathrm{z}:[\mathrm{M}+\mathrm{Na}]^{+}$calcd. for $\mathrm{C}_{28} \mathrm{H}_{22} \mathrm{~N}_{2} \mathrm{O}_{2} \mathrm{Na}$ : 457.1528; found: 457.1529 


\section{X-ray crystallographic structures and data:}

\subsection{X-ray crystallographic structures and data of compound (3d):}

Ellipsoid contour \% probability level $=\mathbf{5 0 \%}$

Experimental: The sample was dissolved in appropriate amount of ethyl acetate followed by the addition of pentane to furnish a saturated solution. Afterwards, the mixture was allowed to stand at room temperature to form the crystals.

Crystal measurements:
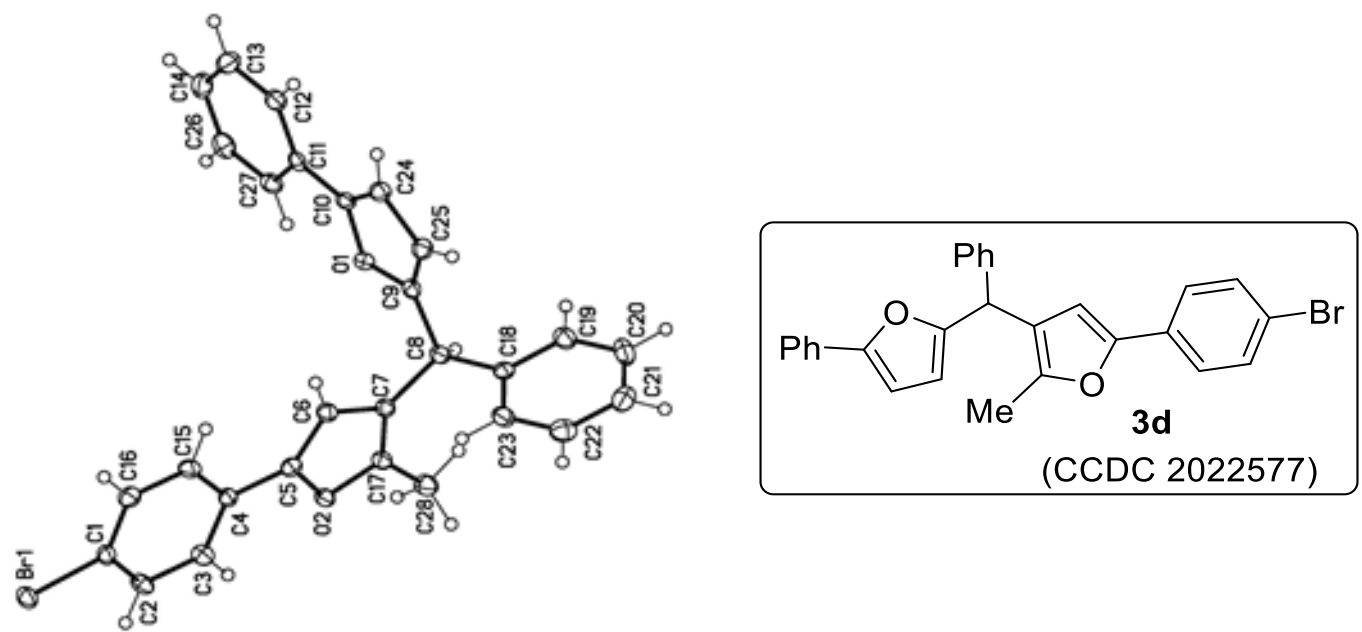

Table S1. Crystal data and structure refinement for 200246lt_0m_a.

Identification code

Empirical formula

Formula weight

Temperature

Wavelength

Crystal system

Space group

Unit cell dimensions

Volume

Z

Density (calculated)

Absorption coefficient
200246LT_0m_a

$\mathrm{C} 28 \mathrm{H} 21 \mathrm{Br} \mathrm{O} 2$

469.36

100(2) K

$0.71073 \AA$

Triclinic

P-1

$a=9.6279(6) \AA$

$\alpha=70.423(2)^{\circ}$.

$\mathrm{b}=9.9867(5) \AA$

$\beta=74.124(2)^{\circ}$.

$\mathrm{c}=12.3249(7) \AA$

$\gamma=84.251(2)^{\circ}$.

1073.91(11) $\AA^{3}$

2

$1.451 \mathrm{Mg} / \mathrm{m}^{3}$

$1.937 \mathrm{~mm}^{-1}$ 
$\mathrm{F}(000)$

Crystal size

Theta range for data collection

Index ranges

Reflections collected

Independent reflections

Completeness to theta $=25.242^{\circ}$

Absorption correction

Max. and min. transmission

Refinement method

Data / restraints / parameters

Goodness-of-fit on $\mathrm{F}^{2}$

Final R indices [I $>2 \operatorname{sigma(I)]~}$

$\mathrm{R}$ indices (all data)

Extinction coefficient

Largest diff. peak and hole
480

$0.15 \times 0.13 \times 0.06 \mathrm{~mm}^{3}$

2.165 to $26.428^{\circ}$.

$-12<=\mathrm{h}<=12,-9<=\mathrm{k}<=12,-14<=1<=15$

18092

$4386[\mathrm{R}(\mathrm{int})=0.0349]$

$99.7 \%$

Semi-empirical from equivalents

0.7454 and 0.6200

Full-matrix least-squares on $\mathrm{F}^{2}$

4386 / 0 / 281

1.090

$\mathrm{R} 1=0.0247, \mathrm{wR} 2=0.0580$

$\mathrm{R} 1=0.0278, w \mathrm{R} 2=0.0593$

$\mathrm{n} / \mathrm{a}$

0.382 and -0.199 e. $\AA^{-3}$ 
Table S2. Atomic coordinates ( $\times 10^{4}$ ) and equivalent isotropic displacement parameters $\left(\AA^{2} \times 10^{3}\right)$ for 2002461t_0m_a. U(eq) is defined as one third of the trace of the orthogonalized $\mathrm{U}^{\mathrm{ij}}$ tensor.

\begin{tabular}{|c|c|c|c|c|}
\hline & $\mathrm{x}$ & $\mathrm{y}$ & $\mathrm{z}$ & $\mathrm{U}(\mathrm{eq})$ \\
\hline $\operatorname{Br}(1)$ & $3460(1)$ & $1536(1)$ & $14000(1)$ & 19(1) \\
\hline $\mathrm{O}(1)$ & 2583(1) & 9106(1) & $6568(1)$ & $15(1)$ \\
\hline $\mathrm{O}(2)$ & $5132(1)$ & $8009(1)$ & $9657(1)$ & $15(1)$ \\
\hline$C(1)$ & $3627(2)$ & $3275(2)$ & $12713(1)$ & $15(1)$ \\
\hline$C(2)$ & $4889(2)$ & $4048(2)$ & $12307(2)$ & $18(1)$ \\
\hline$C(3)$ & 4958(2) & $5361(2)$ & $11431(2)$ & $17(1)$ \\
\hline$C(4)$ & $3789(2)$ & $5908(2)$ & 10949(1) & $14(1)$ \\
\hline$C(5)$ & $3846(2)$ & $7287(2)$ & $10038(1)$ & $15(1)$ \\
\hline$C(6)$ & $2909(2)$ & $8064(2)$ & 9416(1) & $16(1)$ \\
\hline$C(7)$ & $3640(2)$ & $9330(2)$ & $8599(1)$ & $14(1)$ \\
\hline$C(8)$ & 3064(2) & $10522(2)$ & 7690(1) & $15(1)$ \\
\hline $\mathrm{C}(9)$ & $2009(2)$ & $9990(2)$ & $7232(1)$ & $15(1)$ \\
\hline$C(10)$ & $1468(2)$ & $8762(2)$ & 6204(1) & $15(1)$ \\
\hline $\mathrm{C}(11)$ & $1838(2)$ & $7862(2)$ & $5444(1)$ & $15(1)$ \\
\hline$C(12)$ & $762(2)$ & $7456(2)$ & $5055(2)$ & $18(1)$ \\
\hline $\mathrm{C}(13)$ & 1091(2) & $6574(2)$ & $4355(2)$ & $23(1)$ \\
\hline$C(14)$ & 2495(2) & $6086(2)$ & $4035(2)$ & $24(1)$ \\
\hline$C(15)$ & $2543(2)$ & $5087(2)$ & $11361(2)$ & 19(1) \\
\hline$C(16)$ & $2454(2)$ & $3780(2)$ & $12236(2)$ & 19(1) \\
\hline$C(17)$ & 4977(2) & $9253(2)$ & $8777(1)$ & $15(1)$ \\
\hline $\mathrm{C}(18)$ & 2392(2) & $11768(2)$ & $8116(2)$ & $15(1)$ \\
\hline C(19) & $2288(2)$ & $13085(2)$ & $7278(2)$ & $20(1)$ \\
\hline $\mathrm{C}(20)$ & $1708(2)$ & $14250(2)$ & $7622(2)$ & $24(1)$ \\
\hline $\mathrm{C}(21)$ & $1220(2)$ & $14109(2)$ & $8825(2)$ & $24(1)$ \\
\hline$C(22)$ & $1288(2)$ & $12801(2)$ & $9670(2)$ & $23(1)$ \\
\hline $\mathrm{C}(23)$ & 1872(2) & $11631(2)$ & $9319(2)$ & $20(1)$ \\
\hline$C(24)$ & $232(2)$ & $9402(2)$ & $6638(2)$ & $17(1)$ \\
\hline$C(25)$ & $587(2)$ & $10188(2)$ & $7305(2)$ & $17(1)$ \\
\hline$C(26)$ & $3573(2)$ & $6496(2)$ & $4402(2)$ & $22(1)$ \\
\hline $\mathrm{C}(27)$ & $3259(2)$ & $7386(2)$ & $5095(1)$ & $18(1)$ \\
\hline$C(28)$ & $6246(2)$ & $10205(2)$ & $8251(2)$ & $20(1)$ \\
\hline
\end{tabular}


Table S3. Bond lengths [ $[\AA]$ and angles $\left[^{\circ}\right]$ for 200246lt_0m_a.

\begin{tabular}{ll}
\hline $\mathrm{Br}(1)-\mathrm{C}(1)$ & $1.9044(17)$ \\
$\mathrm{O}(1)-\mathrm{C}(9)$ & $1.3769(18)$ \\
$\mathrm{O}(1)-\mathrm{C}(10)$ & $1.3829(19)$ \\
$\mathrm{O}(2)-\mathrm{C}(17)$ & $1.3752(19)$ \\
$\mathrm{O}(2)-\mathrm{C}(5)$ & $1.3815(19)$ \\
$\mathrm{C}(1)-\mathrm{C}(2)$ & $1.386(2)$ \\
$\mathrm{C}(1)-\mathrm{C}(16)$ & $1.387(2)$ \\
$\mathrm{C}(2)-\mathrm{C}(3)$ & $1.385(2)$ \\
$\mathrm{C}(2)-\mathrm{H}(21)$ & 0.9500 \\
$\mathrm{C}(3)-\mathrm{C}(4)$ & $1.395(2)$ \\
$\mathrm{C}(3)-\mathrm{H}(2)$ & 0.9500 \\
$\mathrm{C}(4)-\mathrm{C}(15)$ & $1.398(2)$ \\
$\mathrm{C}(4)-\mathrm{C}(5)$ & $1.451(2)$ \\
$\mathrm{C}(5)-\mathrm{C}(6)$ & $1.358(2)$ \\
$\mathrm{C}(6)-\mathrm{C}(7)$ & $1.430(2)$ \\
$\mathrm{C}(6)-\mathrm{H}(5)$ & 0.9500 \\
$\mathrm{C}(7)-\mathrm{C}(17)$ & $1.354(2)$ \\
$\mathrm{C}(7)-\mathrm{C}(8)$ & $1.515(2)$ \\
$\mathrm{C}(8)-\mathrm{C}(9)$ & $1.502(2)$ \\
$\mathrm{C}(8)-\mathrm{C}(18)$ & $1.527(2)$ \\
$\mathrm{C}(8)-\mathrm{H}(6)$ & 1.0000 \\
$\mathrm{C}(9)-\mathrm{C}(25)$ & $1.346(2)$ \\
$\mathrm{C}(10)-\mathrm{C}(24)$ & $1.356(2)$ \\
$\mathrm{C}(10)-\mathrm{C}(11)$ & $1.459(2)$ \\
$\mathrm{C}(11)-\mathrm{C}(12)$ & $1.398(2)$ \\
$\mathrm{C}(11)-\mathrm{C}(27)$ & $1.403(2)$ \\
$\mathrm{C}(12)-\mathrm{C}(13)$ & $1.387(2)$ \\
$\mathrm{C}(12)-\mathrm{H}(17)$ & 0.9500 \\
$\mathrm{C}(13)-\mathrm{C}(14)$ & $1.387(3)$ \\
$\mathrm{C}(13)-\mathrm{H}(14)$ & 0.9500 \\
$\mathrm{C}(14)-\mathrm{C}(26)$ & \\
& \\
$\mathrm{C}(14)-\mathrm{H}(1)$ & \\
& \\
& \\
&
\end{tabular}




\begin{tabular}{|c|c|}
\hline$C(15)-C(16)$ & $1.380(2)$ \\
\hline $\mathrm{C}(15)-\mathrm{H}(4)$ & 0.9500 \\
\hline $\mathrm{C}(16)-\mathrm{H}(3)$ & 0.9500 \\
\hline $\mathrm{C}(17)-\mathrm{C}(28)$ & $1.484(2)$ \\
\hline $\mathrm{C}(18)-\mathrm{C}(19)$ & $1.388(2)$ \\
\hline $\mathrm{C}(18)-\mathrm{C}(23)$ & $1.393(2)$ \\
\hline$C(19)-C(20)$ & $1.384(2)$ \\
\hline $\mathrm{C}(19)-\mathrm{H}(11)$ & 0.9500 \\
\hline $\mathrm{C}(20)-\mathrm{C}(21)$ & $1.391(3)$ \\
\hline $\mathrm{C}(20)-\mathrm{H}(10)$ & 0.9500 \\
\hline $\mathrm{C}(21)-\mathrm{C}(22)$ & $1.380(3)$ \\
\hline $\mathrm{C}(21)-\mathrm{H}(9)$ & 0.9500 \\
\hline $\mathrm{C}(22)-\mathrm{C}(23)$ & $1.393(2)$ \\
\hline $\mathrm{C}(22)-\mathrm{H}(8)$ & 0.9500 \\
\hline $\mathrm{C}(23)-\mathrm{H}(7)$ & 0.9500 \\
\hline$C(24)-C(25)$ & $1.432(2)$ \\
\hline $\mathrm{C}(24)-\mathrm{H}(13)$ & 0.9500 \\
\hline $\mathrm{C}(25)-\mathrm{H}(12)$ & 0.9500 \\
\hline$C(26)-C(27)$ & $1.387(2)$ \\
\hline $\mathrm{C}(26)-\mathrm{H}(15)$ & 0.9500 \\
\hline $\mathrm{C}(27)-\mathrm{H}(16)$ & 0.9500 \\
\hline $\mathrm{C}(28)-\mathrm{H}(19)$ & 0.9800 \\
\hline $\mathrm{C}(28)-\mathrm{H}(18)$ & 0.9800 \\
\hline $\mathrm{C}(28)-\mathrm{H}(20)$ & 0.9800 \\
\hline $\mathrm{C}(9)-\mathrm{O}(1)-\mathrm{C}(10)$ & $106.87(12)$ \\
\hline $\mathrm{C}(17)-\mathrm{O}(2)-\mathrm{C}(5)$ & $107.07(12)$ \\
\hline $\mathrm{C}(2)-\mathrm{C}(1)-\mathrm{C}(16)$ & $121.07(16)$ \\
\hline $\mathrm{C}(2)-\mathrm{C}(1)-\mathrm{Br}(1)$ & $119.56(13)$ \\
\hline$C(16)-C(1)-B r(1)$ & $119.30(12)$ \\
\hline$C(3)-C(2)-C(1)$ & $119.05(16)$ \\
\hline $\mathrm{C}(3)-\mathrm{C}(2)-\mathrm{H}(21)$ & 120.5 \\
\hline $\mathrm{C}(1)-\mathrm{C}(2)-\mathrm{H}(21)$ & 120.5 \\
\hline$C(2)-C(3)-C(4)$ & $121.19(15)$ \\
\hline $\mathrm{C}(2)-\mathrm{C}(3)-\mathrm{H}(2)$ & 119.4 \\
\hline $\mathrm{C}(4)-\mathrm{C}(3)-\mathrm{H}(2)$ & 119.4 \\
\hline
\end{tabular}




\begin{tabular}{|c|c|}
\hline $\mathrm{C}(3)-\mathrm{C}(4)-\mathrm{C}(15)$ & $118.27(15)$ \\
\hline$C(3)-C(4)-C(5)$ & $121.65(15)$ \\
\hline$C(15)-C(4)-C(5)$ & $120.08(15)$ \\
\hline $\mathrm{C}(6)-\mathrm{C}(5)-\mathrm{O}(2)$ & $109.25(14)$ \\
\hline$C(6)-C(5)-C(4)$ & $134.44(15)$ \\
\hline $\mathrm{O}(2)-\mathrm{C}(5)-\mathrm{C}(4)$ & $116.29(14)$ \\
\hline$C(5)-C(6)-C(7)$ & $107.14(15)$ \\
\hline $\mathrm{C}(5)-\mathrm{C}(6)-\mathrm{H}(5)$ & 126.4 \\
\hline $\mathrm{C}(7)-\mathrm{C}(6)-\mathrm{H}(5)$ & 126.4 \\
\hline$C(17)-C(7)-C(6)$ & $106.58(15)$ \\
\hline$C(17)-C(7)-C(8)$ & $125.96(15)$ \\
\hline$C(6)-C(7)-C(8)$ & $127.45(15)$ \\
\hline $\mathrm{C}(9)-\mathrm{C}(8)-\mathrm{C}(7)$ & $111.71(13)$ \\
\hline $\mathrm{C}(9)-\mathrm{C}(8)-\mathrm{C}(18)$ & $109.51(13)$ \\
\hline$C(7)-C(8)-C(18)$ & $114.74(13)$ \\
\hline $\mathrm{C}(9)-\mathrm{C}(8)-\mathrm{H}(6)$ & 106.8 \\
\hline $\mathrm{C}(7)-\mathrm{C}(8)-\mathrm{H}(6)$ & 106.8 \\
\hline $\mathrm{C}(18)-\mathrm{C}(8)-\mathrm{H}(6)$ & 106.8 \\
\hline $\mathrm{C}(25)-\mathrm{C}(9)-\mathrm{O}(1)$ & $109.97(14)$ \\
\hline $\mathrm{C}(25)-\mathrm{C}(9)-\mathrm{C}(8)$ & $134.67(15)$ \\
\hline $\mathrm{O}(1)-\mathrm{C}(9)-\mathrm{C}(8)$ & $115.35(13)$ \\
\hline $\mathrm{C}(24)-\mathrm{C}(10)-\mathrm{O}(1)$ & $109.37(14)$ \\
\hline$C(24)-C(10)-C(11)$ & $134.00(16)$ \\
\hline $\mathrm{O}(1)-\mathrm{C}(10)-\mathrm{C}(11)$ & $116.61(14)$ \\
\hline $\mathrm{C}(12)-\mathrm{C}(11)-\mathrm{C}(27)$ & $118.89(15)$ \\
\hline$C(12)-C(11)-C(10)$ & $119.78(15)$ \\
\hline $\mathrm{C}(27)-\mathrm{C}(11)-\mathrm{C}(10)$ & $121.33(15)$ \\
\hline $\mathrm{C}(13)-\mathrm{C}(12)-\mathrm{C}(11)$ & $120.44(16)$ \\
\hline $\mathrm{C}(13)-\mathrm{C}(12)-\mathrm{H}(17)$ & 119.8 \\
\hline $\mathrm{C}(11)-\mathrm{C}(12)-\mathrm{H}(17)$ & 119.8 \\
\hline$C(14)-C(13)-C(12)$ & $120.23(16)$ \\
\hline $\mathrm{C}(14)-\mathrm{C}(13)-\mathrm{H}(14)$ & 119.9 \\
\hline $\mathrm{C}(12)-\mathrm{C}(13)-\mathrm{H}(14)$ & 119.9 \\
\hline$C(26)-C(14)-C(13)$ & $119.82(16)$ \\
\hline $\mathrm{C}(26)-\mathrm{C}(14)-\mathrm{H}(1)$ & 120.1 \\
\hline $\mathrm{C}(13)-\mathrm{C}(14)-\mathrm{H}(1)$ & 120.1 \\
\hline
\end{tabular}




\begin{tabular}{|c|c|}
\hline$C(16)-C(15)-C(4)$ & $121.23(16)$ \\
\hline $\mathrm{C}(16)-\mathrm{C}(15)-\mathrm{H}(4)$ & 119.4 \\
\hline $\mathrm{C}(4)-\mathrm{C}(15)-\mathrm{H}(4)$ & 119.4 \\
\hline$C(15)-C(16)-C(1)$ & $119.16(16)$ \\
\hline $\mathrm{C}(15)-\mathrm{C}(16)-\mathrm{H}(3)$ & 120.4 \\
\hline $\mathrm{C}(1)-\mathrm{C}(16)-\mathrm{H}(3)$ & 120.4 \\
\hline $\mathrm{C}(7)-\mathrm{C}(17)-\mathrm{O}(2)$ & $109.96(14)$ \\
\hline $\mathrm{C}(7)-\mathrm{C}(17)-\mathrm{C}(28)$ & $134.23(16)$ \\
\hline $\mathrm{O}(2)-\mathrm{C}(17)-\mathrm{C}(28)$ & $115.81(14)$ \\
\hline$C(19)-C(18)-C(23)$ & $118.55(15)$ \\
\hline $\mathrm{C}(19)-\mathrm{C}(18)-\mathrm{C}(8)$ & $119.10(14)$ \\
\hline $\mathrm{C}(23)-\mathrm{C}(18)-\mathrm{C}(8)$ & $122.36(15)$ \\
\hline C(20)-C(19)-C(18) & $121.14(16)$ \\
\hline $\mathrm{C}(20)-\mathrm{C}(19)-\mathrm{H}(11)$ & 119.4 \\
\hline $\mathrm{C}(18)-\mathrm{C}(19)-\mathrm{H}(11)$ & 119.4 \\
\hline$C(19)-C(20)-C(21)$ & $119.88(17)$ \\
\hline $\mathrm{C}(19)-\mathrm{C}(20)-\mathrm{H}(10)$ & 120.1 \\
\hline $\mathrm{C}(21)-\mathrm{C}(20)-\mathrm{H}(10)$ & 120.1 \\
\hline$C(22)-C(21)-C(20)$ & 119.71(16) \\
\hline $\mathrm{C}(22)-\mathrm{C}(21)-\mathrm{H}(9)$ & 120.1 \\
\hline $\mathrm{C}(20)-\mathrm{C}(21)-\mathrm{H}(9)$ & 120.1 \\
\hline $\mathrm{C}(21)-\mathrm{C}(22)-\mathrm{C}(23)$ & $120.17(17)$ \\
\hline $\mathrm{C}(21)-\mathrm{C}(22)-\mathrm{H}(8)$ & 119.9 \\
\hline $\mathrm{C}(23)-\mathrm{C}(22)-\mathrm{H}(8)$ & 119.9 \\
\hline $\mathrm{C}(22)-\mathrm{C}(23)-\mathrm{C}(18)$ & $120.52(17)$ \\
\hline $\mathrm{C}(22)-\mathrm{C}(23)-\mathrm{H}(7)$ & 119.7 \\
\hline $\mathrm{C}(18)-\mathrm{C}(23)-\mathrm{H}(7)$ & 119.7 \\
\hline$C(10)-C(24)-C(25)$ & $106.83(15)$ \\
\hline $\mathrm{C}(10)-\mathrm{C}(24)-\mathrm{H}(13)$ & 126.6 \\
\hline $\mathrm{C}(25)-\mathrm{C}(24)-\mathrm{H}(13)$ & 126.6 \\
\hline $\mathrm{C}(9)-\mathrm{C}(25)-\mathrm{C}(24)$ & $106.96(14)$ \\
\hline $\mathrm{C}(9)-\mathrm{C}(25)-\mathrm{H}(12)$ & 126.5 \\
\hline $\mathrm{C}(24)-\mathrm{C}(25)-\mathrm{H}(12)$ & 126.5 \\
\hline $\mathrm{C}(14)-\mathrm{C}(26)-\mathrm{C}(27)$ & $120.50(16)$ \\
\hline $\mathrm{C}(14)-\mathrm{C}(26)-\mathrm{H}(15)$ & 119.8 \\
\hline $\mathrm{C}(27)-\mathrm{C}(26)-\mathrm{H}(15)$ & 119.8 \\
\hline
\end{tabular}




\begin{tabular}{ll}
$\mathrm{C}(26)-\mathrm{C}(27)-\mathrm{C}(11)$ & $120.11(16)$ \\
$\mathrm{C}(26)-\mathrm{C}(27)-\mathrm{H}(16)$ & 119.9 \\
$\mathrm{C}(11)-\mathrm{C}(27)-\mathrm{H}(16)$ & 119.9 \\
$\mathrm{C}(17)-\mathrm{C}(28)-\mathrm{H}(19)$ & 109.5 \\
$\mathrm{C}(17)-\mathrm{C}(28)-\mathrm{H}(18)$ & 109.5 \\
$\mathrm{H}(19)-\mathrm{C}(28)-\mathrm{H}(18)$ & 109.5 \\
$\mathrm{C}(17)-\mathrm{C}(28)-\mathrm{H}(20)$ & 109.5 \\
$\mathrm{H}(19)-\mathrm{C}(28)-\mathrm{H}(20)$ & 109.5 \\
$\mathrm{H}(18)-\mathrm{C}(28)-\mathrm{H}(20)$ & 109.5 \\
\hline
\end{tabular}

Symmetry transformations used to generate equivalent atoms: 
Table S4. Anisotropic displacement parameters $\left(\AA^{2} \times 10^{3}\right)$ for 2002461t_0m_a. The anisotropic displacement factor exponent takes the form: $-2 \pi^{2}\left[h^{2} a^{* 2} U^{11}+\ldots+2 h k a^{*} b^{*} U^{12}\right]$

\begin{tabular}{|c|c|c|c|c|c|c|}
\hline & $\mathrm{U}^{11}$ & $\mathrm{U}^{22}$ & $\mathrm{U}^{33}$ & $\mathrm{U}^{23}$ & $\mathrm{U}^{13}$ & $\mathrm{U}^{12}$ \\
\hline $\operatorname{Br}(1)$ & $19(1)$ & $15(1)$ & $18(1)$ & $0(1)$ & $-6(1)$ & $-2(1)$ \\
\hline $\mathrm{O}(1)$ & $15(1)$ & $16(1)$ & $15(1)$ & $-4(1)$ & $-6(1)$ & 1(1) \\
\hline $\mathrm{O}(2)$ & $14(1)$ & $15(1)$ & $15(1)$ & $-2(1)$ & $-5(1)$ & $-1(1)$ \\
\hline $\mathrm{C}(1)$ & $20(1)$ & $12(1)$ & $13(1)$ & $-3(1)$ & $-4(1)$ & $0(1)$ \\
\hline$C(2)$ & $14(1)$ & $20(1)$ & $18(1)$ & $-3(1)$ & $-6(1)$ & $3(1)$ \\
\hline$C(3)$ & $12(1)$ & $19(1)$ & $17(1)$ & $-4(1)$ & $-3(1)$ & $-1(1)$ \\
\hline$C(4)$ & $16(1)$ & $13(1)$ & $13(1)$ & $-4(1)$ & $-4(1)$ & 1(1) \\
\hline$C(5)$ & $14(1)$ & $17(1)$ & $16(1)$ & $-6(1)$ & $-3(1)$ & $-1(1)$ \\
\hline$C(6)$ & $13(1)$ & $16(1)$ & $18(1)$ & $-4(1)$ & $-6(1)$ & $0(1)$ \\
\hline$C(7)$ & $15(1)$ & $14(1)$ & $13(1)$ & $-4(1)$ & $-4(1)$ & $0(1)$ \\
\hline$C(8)$ & $15(1)$ & $15(1)$ & $14(1)$ & $-2(1)$ & $-5(1)$ & $-1(1)$ \\
\hline $\mathrm{C}(9)$ & $20(1)$ & $12(1)$ & $12(1)$ & $-2(1)$ & $-5(1)$ & $-1(1)$ \\
\hline$C(10)$ & $17(1)$ & $13(1)$ & $14(1)$ & $1(1)$ & $-7(1)$ & $-4(1)$ \\
\hline$C(11)$ & 19(1) & 11(1) & $12(1)$ & $1(1)$ & $-5(1)$ & $-1(1)$ \\
\hline$C(12)$ & $18(1)$ & $17(1)$ & $19(1)$ & $-2(1)$ & $-7(1)$ & $0(1)$ \\
\hline $\mathrm{C}(13)$ & $27(1)$ & $22(1)$ & $22(1)$ & $-7(1)$ & $-8(1)$ & $-5(1)$ \\
\hline$C(14)$ & $34(1)$ & $17(1)$ & $20(1)$ & $-7(1)$ & $-6(1)$ & 1(1) \\
\hline$C(15)$ & $18(1)$ & $19(1)$ & $23(1)$ & $-4(1)$ & $-12(1)$ & $-1(1)$ \\
\hline$C(16)$ & $17(1)$ & $19(1)$ & $22(1)$ & $-4(1)$ & $-7(1)$ & $-5(1)$ \\
\hline $\mathrm{C}(17)$ & $18(1)$ & $15(1)$ & $12(1)$ & $-4(1)$ & $-5(1)$ & $0(1)$ \\
\hline$C(18)$ & $12(1)$ & $16(1)$ & $19(1)$ & $-4(1)$ & $-7(1)$ & $-2(1)$ \\
\hline C(19) & $19(1)$ & $19(1)$ & $20(1)$ & $-2(1)$ & $-6(1)$ & $-2(1)$ \\
\hline $\mathrm{C}(20)$ & 19(1) & $15(1)$ & $33(1)$ & $0(1)$ & $-8(1)$ & $0(1)$ \\
\hline $\mathrm{C}(21)$ & $14(1)$ & $23(1)$ & $38(1)$ & $-16(1)$ & $-9(1)$ & $3(1)$ \\
\hline $\mathrm{C}(22)$ & 19(1) & $30(1)$ & $24(1)$ & $-12(1)$ & $-7(1)$ & $4(1)$ \\
\hline $\mathrm{C}(23)$ & $20(1)$ & $19(1)$ & $20(1)$ & $-4(1)$ & $-9(1)$ & 1(1) \\
\hline $\mathrm{C}(24)$ & $16(1)$ & $17(1)$ & $18(1)$ & $-4(1)$ & $-6(1)$ & $-2(1)$ \\
\hline $\mathrm{C}(25)$ & $17(1)$ & $17(1)$ & $17(1)$ & $-6(1)$ & $-4(1)$ & 1(1) \\
\hline$C(26)$ & 21(1) & $20(1)$ & $19(1)$ & $-3(1)$ & $-1(1)$ & $3(1)$ \\
\hline $\mathrm{C}(27)$ & $17(1)$ & $18(1)$ & $15(1)$ & $-1(1)$ & $-5(1)$ & $-2(1)$ \\
\hline \multirow[t]{2}{*}{$C(28)$} & 19(1) & $21(1)$ & $18(1)$ & $-1(1)$ & $-6(1)$ & $-4(1)$ \\
\hline & & & & \multicolumn{3}{|l|}{ S32 } \\
\hline
\end{tabular}


Table S5. Hydrogen coordinates ( $\left.\mathrm{x} 10^{4}\right)$ and isotropic displacement parameters $\left(\AA^{2} \times 10^{3}\right)$ for 200246lt_0m_a.

\begin{tabular}{|c|c|c|c|c|}
\hline & $\mathrm{x}$ & $\mathrm{y}$ & $\mathrm{z}$ & $\mathrm{U}(\mathrm{eq})$ \\
\hline $\mathrm{H}(21)$ & 5695 & 3683 & 12625 & 21 \\
\hline $\mathrm{H}(2)$ & 5819 & 5900 & 11154 & 20 \\
\hline $\mathrm{H}(5)$ & 1947 & 7815 & 9508 & 19 \\
\hline $\mathrm{H}(6)$ & 3906 & 10907 & 6995 & 18 \\
\hline $\mathrm{H}(17)$ & -201 & 7786 & 5272 & 22 \\
\hline $\mathrm{H}(14)$ & 354 & 6303 & 4095 & 27 \\
\hline $\mathrm{H}(1)$ & 2715 & 5471 & 3564 & 29 \\
\hline $\mathrm{H}(4)$ & 1742 & 5436 & 11032 & 23 \\
\hline $\mathrm{H}(3)$ & 1601 & 3232 & 12509 & 23 \\
\hline $\mathrm{H}(11)$ & 2622 & 13189 & 6454 & 24 \\
\hline $\mathrm{H}(10)$ & 1642 & 15143 & 7036 & 29 \\
\hline $\mathrm{H}(9)$ & 841 & 14911 & 9065 & 28 \\
\hline $\mathrm{H}(8)$ & 937 & 12697 & 10493 & 28 \\
\hline $\mathrm{H}(7)$ & 1916 & 10733 & 9905 & 23 \\
\hline $\mathrm{H}(13)$ & -692 & 9340 & 6521 & 20 \\
\hline $\mathrm{H}(12)$ & -58 & 10744 & 7719 & 21 \\
\hline $\mathrm{H}(15)$ & 4535 & 6166 & 4178 & 26 \\
\hline $\mathrm{H}(16)$ & 4008 & 7673 & 5334 & 21 \\
\hline $\mathrm{H}(19)$ & 6120 & 10938 & 7521 & 30 \\
\hline $\mathrm{H}(18)$ & 7122 & 9649 & 8062 & 30 \\
\hline $\mathrm{H}(20)$ & 6332 & 10657 & 8823 & 30 \\
\hline
\end{tabular}




\subsection{X-ray crystallographic structures and data of compound (5j):}

Ellipsoid contour \% probability level $=\mathbf{5 0} \%$

Experimental: The sample was dissolved in appropriate amount of ethyl acetate followed by the addition of pentane to furnish a saturated solution. Afterwards, the mixture was allowed to stand at room temperature to form the crystals.

\section{Crystal measurements:}
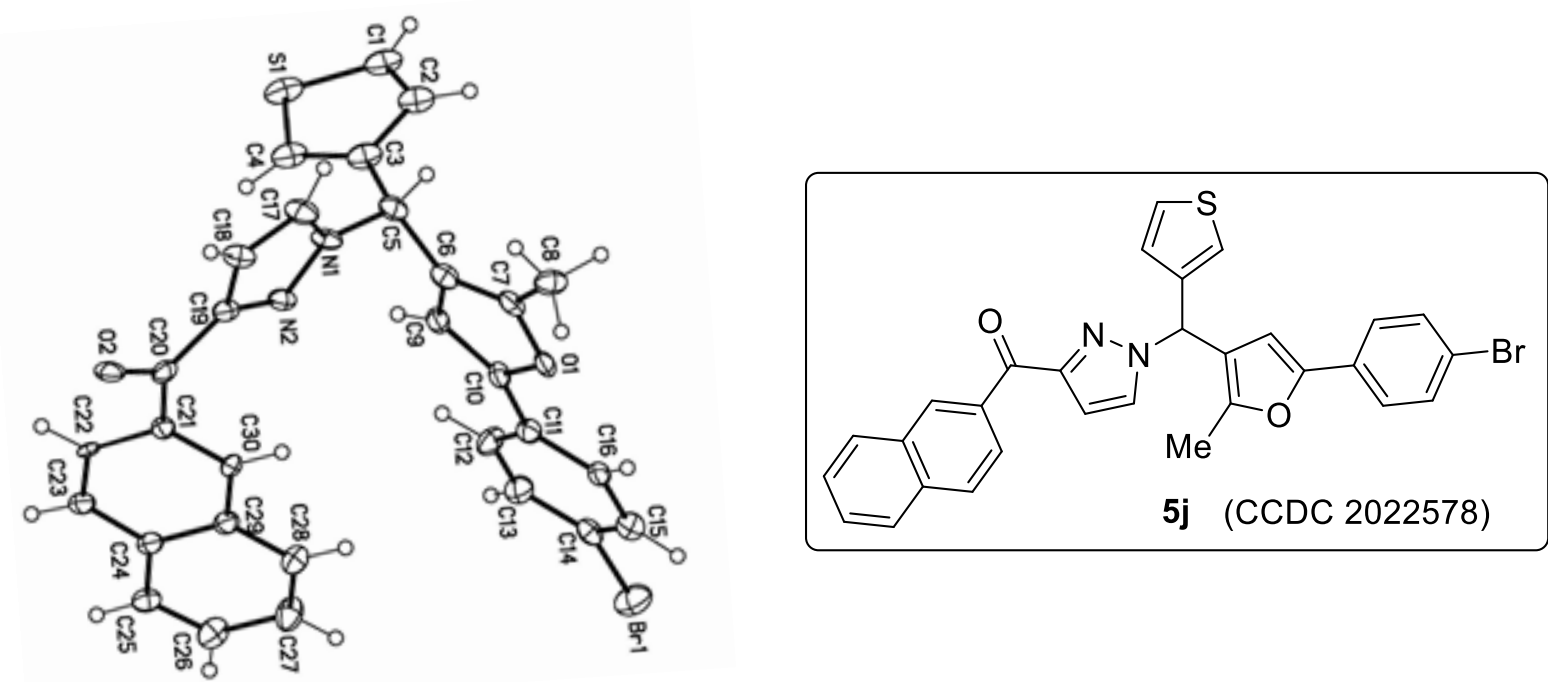

Table S6. Crystal data and structure refinement for twin5.

Identification code

Empirical formula

Formula weight

Temperature

Wavelength

Crystal system

Space group

Unit cell dimensions

Volume

Z

Density (calculated)

Absorption coefficient twin5

C30 H21 Br N2 O2 S

553.46

100(2) K

$0.71073 \AA$

Triclinic

P-1

$\mathrm{a}=9.4923(8) \AA \quad \alpha=86.150(3)^{\circ}$.

$\mathrm{b}=10.6838(10) \AA \quad \beta=88.487(3)^{\circ}$.

$\mathrm{c}=27.294(2) \AA \quad \gamma=64.009(3)^{\circ}$.

2482.4(4) $\AA^{3}$

4

$1.481 \mathrm{Mg} / \mathrm{m}^{3}$

$1.772 \mathrm{~mm}^{-1}$ 
$\mathrm{F}(000)$

Crystal size

Theta range for data collection

Index ranges

Reflections collected

Independent reflections

Completeness to theta $=25.242^{\circ}$

Absorption correction

Max. and min. transmission

Refinement method

Data / restraints / parameters

Goodness-of-fit on $\mathrm{F}^{2}$

Final $\mathrm{R}$ indices [I>2sigma(I)]

$\mathrm{R}$ indices (all data)

Extinction coefficient

Largest diff. peak and hole
1128

$0.15 \times 0.12 \times 0.08 \mathrm{~mm}^{3}$

0.748 to $26.405^{\circ}$.

$-11<=\mathrm{h}<=11,-13<=\mathrm{k}<=13,0<=\mathrm{l}<=34$

7310

$7310[\mathrm{R}(\mathrm{int})=0.0432]$

$81.0 \%$

Semi-empirical from equivalents

0.7454 and 0.7059

Full-matrix least-squares on $\mathrm{F}^{2}$

7310 / 1050 / 744

1.079

$\mathrm{R} 1=0.0942, \mathrm{wR} 2=0.2973$

$\mathrm{R} 1=0.1134, \mathrm{wR} 2=0.3071$

$\mathrm{n} / \mathrm{a}$

1.303 and -0.934 e. $\AA^{-3}$ 
Table S7. Atomic coordinates ( $\times 10^{4}$ ) and equivalent isotropic displacement parameters $\left(\AA^{2} \times 10^{3}\right)$ for twin5. $U(\mathrm{eq})$ is defined as one third of the trace of the orthogonalized $\mathrm{U}^{\mathrm{ij}}$ tensor.

\begin{tabular}{|c|c|c|c|c|}
\hline & $\mathrm{x}$ & $\mathrm{y}$ & $\mathrm{z}$ & $\mathrm{U}(\mathrm{eq})$ \\
\hline $\operatorname{Br}(1)$ & $8285(2)$ & $-2992(2)$ & $11824(1)$ & $35(1)$ \\
\hline $\operatorname{Br}(2)$ & $3086(2)$ & $3110(2)$ & $3145(1)$ & $42(1)$ \\
\hline $\mathrm{S}(1)$ & 5063(5) & $-1407(5)$ & $8000(2)$ & $29(1)$ \\
\hline$C(1)$ & $6910(20)$ & $-2840(20)$ & $8042(7)$ & $27(2)$ \\
\hline$C(2)$ & $8000(20)$ & $-2464(18)$ & $8139(7)$ & $27(2)$ \\
\hline$C(3)$ & $7420(20)$ & $-990(20)$ & $8200(7)$ & $25(2)$ \\
\hline$C(4)$ & $5830(20)$ & $-287(18)$ & $8122(6)$ & $26(2)$ \\
\hline$S\left(1^{\prime}\right)$ & $6380(100)$ & $-2650(90)$ & $8000(30)$ & $27(3)$ \\
\hline$C\left(1^{\prime}\right)$ & $5140(130)$ & $-930(130)$ & $8140(90)$ & $27(3)$ \\
\hline $\mathrm{C}\left(2^{\prime}\right)$ & $6000(200)$ & $-300(150)$ & $8260(90)$ & $27(3)$ \\
\hline$C\left(3^{\prime}\right)$ & $7600(200)$ & $-1000(200)$ & $8130(120)$ & $26(3)$ \\
\hline$C\left(4^{\prime}\right)$ & 7930(140) & $-2270(170)$ & 7930(90) & $26(3)$ \\
\hline $\mathrm{S}(2)$ & $2265(15)$ & $621(14)$ & 7035(4) & $38(2)$ \\
\hline $\mathrm{C}(31)$ & $3560(50)$ & $1360(40)$ & $6973(18)$ & $35(3)$ \\
\hline $\mathrm{C}(32)$ & $2670(90)$ & $2840(80)$ & $6810(30)$ & $32(3)$ \\
\hline $\mathrm{C}(33)$ & $1160(50)$ & $3210(40)$ & $6729(15)$ & $33(3)$ \\
\hline$C(34)$ & $710(40)$ & $2060(50)$ & $6820(20)$ & $35(3)$ \\
\hline$S\left(2^{\prime}\right)$ & $379(11)$ & 2401(11) & 6918(3) & $37(2)$ \\
\hline$C\left(31^{\prime}\right)$ & $1050(40)$ & $3650(30)$ & $6816(12)$ & $35(3)$ \\
\hline$C\left(32^{\prime}\right)$ & $2770(80)$ & $2940(70)$ & $6780(20)$ & $32(3)$ \\
\hline$C\left(33^{\prime}\right)$ & $3360(40)$ & $1570(40)$ & 6884(13) & $34(3)$ \\
\hline$C\left(34^{\prime}\right)$ & $2170(40)$ & $1040(40)$ & $6922(15)$ & $37(3)$ \\
\hline $\mathrm{C}(5)$ & $8507(17)$ & $-348(15)$ & $8337(5)$ & $20(2)$ \\
\hline$C(6)$ & $8956(17)$ & $-677(14)$ & $8877(5)$ & $20(2)$ \\
\hline$C(7)$ & 10411(17) & $-1008(15)$ & $9057(6)$ & 20(3) \\
\hline $\mathrm{C}(8)$ & $11890(18)$ & $-1055(16)$ & $8821(6)$ & $23(3)$ \\
\hline $\mathrm{C}(9)$ & $8058(16)$ & $-779(14)$ & $9277(5)$ & $18(2)$ \\
\hline $\mathrm{C}(10)$ & $8979(16)$ & $-1126(14)$ & 9694(5) & $18(2)$ \\
\hline $\mathrm{C}(11)$ & $8795(16)$ & $-1501(14)$ & $10201(5)$ & 19(3) \\
\hline $\mathrm{C}(12)$ & $7409(17)$ & $-1495(16)$ & $10366(6)$ & $22(3)$ \\
\hline $\mathrm{C}(13)$ & 7233(19) & $-1913(16)$ & $10830(6)$ & $25(3)$ \\
\hline
\end{tabular}




\begin{tabular}{|c|c|c|c|c|}
\hline$C(14)$ & $8475(18)$ & $-2372(15)$ & $11166(6)$ & $22(3)$ \\
\hline$C(15)$ & 9874(19) & $-2375(17)$ & $11032(6)$ & $27(3)$ \\
\hline$C(16)$ & $10022(16)$ & $-1950(15)$ & $10541(5)$ & $19(3)$ \\
\hline $\mathrm{C}(17)$ & $8123(18)$ & $1813(15)$ & 7833(5) & $24(3)$ \\
\hline $\mathrm{C}(18)$ & $7233(17)$ & $3201(15)$ & $7855(5)$ & $22(3)$ \\
\hline $\mathrm{C}(19)$ & $6282(16)$ & $3329(14)$ & $8273(5)$ & $17(2)$ \\
\hline$C(20)$ & $5056(16)$ & $4587(15)$ & $8468(5)$ & $17(2)$ \\
\hline $\mathrm{C}(21)$ & $4267(15)$ & 4541(14) & $8934(5)$ & $16(2)$ \\
\hline$C(22)$ & $2632(15)$ & $5427(13)$ & $8973(5)$ & $12(2)$ \\
\hline$C(23)$ & 1831(17) & $5483(15)$ & 9393(5) & 19(3) \\
\hline$C(24)$ & $2625(16)$ & $4714(15)$ & $9823(5)$ & $18(2)$ \\
\hline$C(25)$ & $1823(17)$ & $4732(15)$ & $10279(5)$ & $20(3)$ \\
\hline$C(26)$ & $2608(17)$ & $3920(16)$ & $10667(6)$ & $24(3)$ \\
\hline$C(27)$ & $4241(17)$ & $3021(16)$ & $10652(6)$ & $27(3)$ \\
\hline$C(28)$ & $5052(17)$ & 2987(15) & $10221(5)$ & $21(3)$ \\
\hline$C(29)$ & $4264(16)$ & $3800(14)$ & $9800(5)$ & $18(2)$ \\
\hline$C(30)$ & $5053(15)$ & $3738(13)$ & $9356(5)$ & $13(2)$ \\
\hline$C(35)$ & $3662(18)$ & $3692(18)$ & $6676(6)$ & $28(3)$ \\
\hline$C(36)$ & $3280(20)$ & $5600(20)$ & 7244(7) & $44(4)$ \\
\hline$C(37)$ & $2260(20)$ & $6980(20)$ & $7254(6)$ & $42(4)$ \\
\hline$C(38)$ & $1300(20)$ & $7274(18)$ & 6844(6) & $37(4)$ \\
\hline C(39) & $-1(19)$ & $8623(18)$ & 6706(6) & $35(4)$ \\
\hline $\mathrm{C}(40)$ & $-758(18)$ & $8909(16)$ & $6209(6)$ & $28(3)$ \\
\hline $\mathrm{C}(41)$ & $-2388(19)$ & $9817(16)$ & 6178(6) & $28(3)$ \\
\hline $\mathrm{C}(42)$ & $-3150(20)$ & 10131(16) & $5737(6)$ & $30(3)$ \\
\hline $\mathrm{C}(43)$ & $-2348(19)$ & $9586(16)$ & $5304(6)$ & $28(3)$ \\
\hline $\mathrm{C}(44)$ & $-3090(20)$ & 9902(17) & $4849(6)$ & $33(3)$ \\
\hline $\mathrm{C}(45)$ & $-2260(20)$ & $9298(18)$ & $4435(7)$ & $35(4)$ \\
\hline $\mathrm{C}(46)$ & $-650(20)$ & $8360(20)$ & $4460(7)$ & $47(5)$ \\
\hline $\mathrm{C}(47)$ & $90(20)$ & $8096(19)$ & 4905(6) & $35(4)$ \\
\hline $\mathrm{C}(48)$ & $-684(18)$ & $8675(16)$ & $5342(6)$ & $26(3)$ \\
\hline C(49) & $31(19)$ & $8355(16)$ & $5795(6)$ & $28(3)$ \\
\hline$C(50)$ & $5545(17)$ & $3472(16)$ & $5958(5)$ & $21(3)$ \\
\hline$C(51)$ & $4082(17)$ & $3699(15)$ & $6141(5)$ & $22(3)$ \\
\hline$C(52)$ & $3109(18)$ & $3806(15)$ & $5725(5)$ & $22(3)$ \\
\hline$C(53)$ & $4035(17)$ & $3664(14)$ & $5315(5)$ & $20(3)$ \\
\hline
\end{tabular}




\begin{tabular}{lrlll}
$\mathrm{C}(54)$ & $3781(17)$ & $3604(15)$ & $4800(5)$ & $20(3)$ \\
$\mathrm{C}(55)$ & $2347(19)$ & $3728(16)$ & $4626(6)$ & $25(3)$ \\
$\mathrm{C}(56)$ & $2130(20)$ & $3623(17)$ & $4137(6)$ & $29(3)$ \\
$\mathrm{C}(57)$ & $3360(20)$ & $3316(16)$ & $3808(6)$ & $26(3)$ \\
$\mathrm{C}(58)$ & $4830(20)$ & $3167(18)$ & $3970(6)$ & $30(3)$ \\
$\mathrm{C}(59)$ & $5013(18)$ & $3314(15)$ & $4447(5)$ & $23(3)$ \\
$\mathrm{C}(60)$ & $6983(18)$ & $3285(19)$ & $6191(6)$ & $28(4)$ \\
$\mathrm{N}(1)$ & $7798(14)$ & $1156(12)$ & $8218(4)$ & $19(2)$ \\
$\mathrm{N}(2)$ & $6666(13)$ & $2035(12)$ & $8500(4)$ & $18(2)$ \\
$\mathrm{N}(3)$ & $2905(14)$ & $5126(15)$ & $6835(5)$ & $28(3)$ \\
$\mathrm{N}(4)$ & $1695(15)$ & $6150(15)$ & $6573(5)$ & $32(3)$ \\
$\mathrm{O}(1)$ & $10459(10)$ & $-1251(9)$ & $9549(4)$ & $18(2)$ \\
$\mathrm{O}(2)$ & $4633(12)$ & $5697(10)$ & $8211(3)$ & $21(2)$ \\
$\mathrm{O}(3)$ & $5502(11)$ & $3492(10)$ & $5452(4)$ & $21(2)$ \\
$\mathrm{O}(4)$ & $-462(15)$ & $9569(14)$ & $7004(5)$ & $51(3)$ \\
\hline
\end{tabular}


Table S8. Bond lengths $[\AA]$ and angles $\left[{ }^{\circ}\right]$ for twin5.

\begin{tabular}{|c|c|}
\hline $\operatorname{Br}(1)-C(14)$ & $1.903(16)$ \\
\hline $\mathrm{Br}(2)-\mathrm{C}(57)$ & $1.876(16)$ \\
\hline$S(1)-C(4)$ & $1.702(17)$ \\
\hline$S(1)-C(1)$ & $1.75(2)$ \\
\hline $\mathrm{C}(1)-\mathrm{C}(2)$ & $1.30(3)$ \\
\hline $\mathrm{C}(1)-\mathrm{H}(1)$ & 0.9500 \\
\hline$C(2)-C(3)$ & $1.45(2)$ \\
\hline $\mathrm{C}(2)-\mathrm{H}(2)$ & 0.9500 \\
\hline $\mathrm{C}(3)-\mathrm{C}(4)$ & $1.38(2)$ \\
\hline$C(3)-C(5)$ & $1.53(2)$ \\
\hline $\mathrm{C}(4)-\mathrm{H}(4)$ & 0.9500 \\
\hline$S\left(1^{\prime}\right)-C\left(4^{\prime}\right)$ & $1.70(3)$ \\
\hline$S\left(1^{\prime}\right)-C\left(1^{\prime}\right)$ & $1.75(3)$ \\
\hline $\mathrm{C}\left(1^{\prime}\right)-\mathrm{C}\left(2^{\prime}\right)$ & $1.31(3)$ \\
\hline C(1')-H(1') & 0.9500 \\
\hline $\mathrm{C}\left(2^{\prime}\right)-\mathrm{C}\left(3^{\prime}\right)$ & $1.45(3)$ \\
\hline $\mathrm{C}\left(2^{\prime}\right)-\mathrm{H}\left(2^{\prime}\right)$ & 0.9500 \\
\hline $\mathrm{C}\left(3^{\prime}\right)-\mathrm{C}\left(4^{\prime}\right)$ & $1.38(3)$ \\
\hline$C\left(3^{\prime}\right)-C(5)$ & $1.48(11)$ \\
\hline $\mathrm{C}\left(4^{\prime}\right)-\mathrm{H}\left(4^{\prime}\right)$ & 0.9500 \\
\hline$S(2)-C(34)$ & $1.68(4)$ \\
\hline$S(2)-C(31)$ & $1.72(3)$ \\
\hline $\mathrm{C}(31)-\mathrm{C}(32)$ & $1.47(8)$ \\
\hline $\mathrm{C}(31)-\mathrm{H}(31)$ & 0.9500 \\
\hline $\mathrm{C}(32)-\mathrm{C}(33)$ & $1.33(7)$ \\
\hline$C(32)-C(35)$ & $1.59(3)$ \\
\hline C(33)-C(34) & $1.47(5)$ \\
\hline C(33)-H(33) & 0.9500 \\
\hline C(34)-H(34) & 0.9500 \\
\hline$S\left(2^{\prime}\right)-C\left(34^{\prime}\right)$ & $1.69(3)$ \\
\hline$S\left(2^{\prime}\right)-C\left(31^{\prime}\right)$ & $1.72(3)$ \\
\hline $\mathrm{C}\left(31^{\prime}\right)-\mathrm{C}\left(32^{\prime}\right)$ & $1.47(8)$ \\
\hline $\mathrm{C}\left(31^{\prime}\right)-\mathrm{H}\left(31^{\prime}\right)$ & 0.9500 \\
\hline $\mathrm{C}\left(32^{\prime}\right)-\mathrm{C}\left(33^{\prime}\right)$ & $1.33(7)$ \\
\hline
\end{tabular}




\begin{tabular}{|c|c|}
\hline C(32')-C(35) & $1.42(3)$ \\
\hline $\mathrm{C}\left(33^{\prime}\right)-\mathrm{C}\left(34^{\prime}\right)$ & $1.47(4)$ \\
\hline $\mathrm{C}\left(33^{\prime}\right)-\mathrm{H}\left(33^{\prime}\right)$ & 0.9500 \\
\hline $\mathrm{C}\left(34^{\prime}\right)-\mathrm{H}\left(34^{\prime}\right)$ & 0.9500 \\
\hline $\mathrm{C}(5)-\mathrm{N}(1)$ & $1.462(18)$ \\
\hline$C(5)-C(6)$ & $1.52(2)$ \\
\hline $\mathrm{C}(5)-\mathrm{H}(5)$ & 1.0000 \\
\hline$C(6)-C(7)$ & $1.36(2)$ \\
\hline$C(6)-C(9)$ & $1.40(2)$ \\
\hline $\mathrm{C}(7)-\mathrm{O}(1)$ & $1.349(17)$ \\
\hline$C(7)-C(8)$ & $1.51(2)$ \\
\hline $\mathrm{C}(8)-\mathrm{H}(8 \mathrm{~A})$ & 0.9800 \\
\hline $\mathrm{C}(8)-\mathrm{H}(8 \mathrm{~B})$ & 0.9800 \\
\hline $\mathrm{C}(8)-\mathrm{H}(8 \mathrm{C})$ & 0.9800 \\
\hline C(9)-C(10) & $1.380(19)$ \\
\hline $\mathrm{C}(9)-\mathrm{H}(9)$ & 0.9500 \\
\hline $\mathrm{C}(10)-\mathrm{O}(1)$ & $1.401(16)$ \\
\hline $\mathrm{C}(10)-\mathrm{C}(11)$ & $1.44(2)$ \\
\hline $\mathrm{C}(11)-\mathrm{C}(12)$ & $1.38(2)$ \\
\hline $\mathrm{C}(11)-\mathrm{C}(16)$ & $1.398(19)$ \\
\hline $\mathrm{C}(12)-\mathrm{C}(13)$ & $1.35(2)$ \\
\hline $\mathrm{C}(12)-\mathrm{H}(12)$ & 0.9500 \\
\hline C(13)-C(14) & $1.40(2)$ \\
\hline $\mathrm{C}(13)-\mathrm{H}(13)$ & 0.9500 \\
\hline$C(14)-C(15)$ & $1.37(2)$ \\
\hline$C(15)-C(16)$ & $1.41(2)$ \\
\hline $\mathrm{C}(15)-\mathrm{H}(15)$ & 0.9500 \\
\hline $\mathrm{C}(16)-\mathrm{H}(16)$ & 0.9500 \\
\hline $\mathrm{C}(17)-\mathrm{N}(1)$ & $1.328(18)$ \\
\hline $\mathrm{C}(17)-\mathrm{C}(18)$ & $1.35(2)$ \\
\hline $\mathrm{C}(17)-\mathrm{H}(17)$ & 0.9500 \\
\hline C(18)-C(19) & $1.413(19)$ \\
\hline C(18)-H(18) & 0.9500 \\
\hline $\mathrm{C}(19)-\mathrm{N}(2)$ & $1.373(18)$ \\
\hline C(19)-C(20) & $1.462(19)$ \\
\hline $\mathrm{C}(20)-\mathrm{O}(2)$ & $1.243(17)$ \\
\hline
\end{tabular}




\begin{tabular}{|c|c|}
\hline$C(20)-C(21)$ & $1.467(19)$ \\
\hline$C(21)-C(30)$ & $1.405(18)$ \\
\hline $\mathrm{C}(21)-\mathrm{C}(22)$ & $1.426(18)$ \\
\hline $\mathrm{C}(22)-\mathrm{C}(23)$ & $1.350(19)$ \\
\hline $\mathrm{C}(22)-\mathrm{H}(22)$ & 0.9500 \\
\hline$C(23)-C(24)$ & 1.411(19) \\
\hline $\mathrm{C}(23)-\mathrm{H}(23)$ & 0.9500 \\
\hline$C(24)-C(29)$ & $1.43(2)$ \\
\hline$C(24)-C(25)$ & $1.439(19)$ \\
\hline$C(25)-C(26)$ & $1.33(2)$ \\
\hline $\mathrm{C}(25)-\mathrm{H}(25)$ & 0.9500 \\
\hline$C(26)-C(27)$ & $1.42(2)$ \\
\hline $\mathrm{C}(26)-\mathrm{H}(26)$ & 0.9500 \\
\hline $\mathrm{C}(27)-\mathrm{C}(28)$ & $1.38(2)$ \\
\hline $\mathrm{C}(27)-\mathrm{H}(27)$ & 0.9500 \\
\hline C(28)-C(29) & $1.406(19)$ \\
\hline $\mathrm{C}(28)-\mathrm{H}(28)$ & 0.9500 \\
\hline C(29)-C(30) & $1.397(19)$ \\
\hline $\mathrm{C}(30)-\mathrm{H}(30)$ & 0.9500 \\
\hline $\mathrm{C}(35)-\mathrm{N}(3)$ & $1.47(2)$ \\
\hline $\mathrm{C}(35)-\mathrm{C}(51)$ & $1.50(2)$ \\
\hline $\mathrm{C}(35)-\mathrm{H}(35)$ & 1.0000 \\
\hline C(36)-C(37) & $1.36(3)$ \\
\hline $\mathrm{C}(36)-\mathrm{N}(3)$ & $1.38(2)$ \\
\hline $\mathrm{C}(36)-\mathrm{H}(36)$ & 0.9500 \\
\hline $\mathrm{C}(37)-\mathrm{C}(38)$ & $1.40(2)$ \\
\hline $\mathrm{C}(37)-\mathrm{H}(37)$ & 0.9500 \\
\hline $\mathrm{C}(38)-\mathrm{N}(4)$ & $1.357(19)$ \\
\hline $\mathrm{C}(38)-\mathrm{C}(39)$ & $1.46(2)$ \\
\hline $\mathrm{C}(39)-\mathrm{O}(4)$ & $1.258(19)$ \\
\hline $\mathrm{C}(39)-\mathrm{C}(40)$ & $1.50(2)$ \\
\hline $\mathrm{C}(40)-\mathrm{C}(49)$ & $1.36(2)$ \\
\hline $\mathrm{C}(40)-\mathrm{C}(41)$ & $1.42(2)$ \\
\hline $\mathrm{C}(41)-\mathrm{C}(42)$ & $1.37(2)$ \\
\hline $\mathrm{C}(41)-\mathrm{H}(41)$ & 0.9500 \\
\hline $\mathrm{C}(42)-\mathrm{C}(43)$ & $1.40(2)$ \\
\hline
\end{tabular}




\begin{tabular}{|c|c|}
\hline $\mathrm{C}(42)-\mathrm{H}(42)$ & 0.9500 \\
\hline $\mathrm{C}(43)-\mathrm{C}(44)$ & $1.39(2)$ \\
\hline $\mathrm{C}(43)-\mathrm{C}(48)$ & $1.45(2)$ \\
\hline C(44)-C(45) & $1.39(2)$ \\
\hline $\mathrm{C}(44)-\mathrm{H}(44)$ & 0.9500 \\
\hline C(45)-C(46) & $1.41(2)$ \\
\hline $\mathrm{C}(45)-\mathrm{H}(45)$ & 0.9500 \\
\hline $\mathrm{C}(46)-\mathrm{C}(47)$ & $1.37(2)$ \\
\hline $\mathrm{C}(46)-\mathrm{H}(46)$ & 0.9500 \\
\hline $\mathrm{C}(47)-\mathrm{C}(48)$ & $1.42(2)$ \\
\hline $\mathrm{C}(47)-\mathrm{H}(47)$ & 0.9500 \\
\hline C(48)-C(49) & $1.37(2)$ \\
\hline C(49)-H(49) & 0.9500 \\
\hline $\mathrm{C}(50)-\mathrm{O}(3)$ & $1.379(17)$ \\
\hline $\mathrm{C}(50)-\mathrm{C}(51)$ & $1.39(2)$ \\
\hline$C(50)-C(60)$ & $1.45(2)$ \\
\hline $\mathrm{C}(51)-\mathrm{C}(52)$ & $1.45(2)$ \\
\hline $\mathrm{C}(52)-\mathrm{C}(53)$ & $1.378(19)$ \\
\hline $\mathrm{C}(52)-\mathrm{H}(52)$ & 0.9500 \\
\hline $\mathrm{C}(53)-\mathrm{O}(3)$ & $1.381(17)$ \\
\hline C(53)-C(54) & $1.444(19)$ \\
\hline $\mathrm{C}(54)-\mathrm{C}(55)$ & $1.40(2)$ \\
\hline $\mathrm{C}(54)-\mathrm{C}(59)$ & $1.43(2)$ \\
\hline$C(55)-C(56)$ & $1.38(2)$ \\
\hline $\mathrm{C}(55)-\mathrm{H}(55)$ & 0.9500 \\
\hline$C(56)-C(57)$ & $1.39(2)$ \\
\hline $\mathrm{C}(56)-\mathrm{H}(56)$ & 0.9500 \\
\hline $\mathrm{C}(57)-\mathrm{C}(58)$ & $1.41(2)$ \\
\hline $\mathrm{C}(58)-\mathrm{C}(59)$ & $1.35(2)$ \\
\hline C(58)-H(58) & 0.9500 \\
\hline C(59)-H(59) & 0.9500 \\
\hline $\mathrm{C}(60)-\mathrm{H}(60 \mathrm{~A})$ & 0.9800 \\
\hline $\mathrm{C}(60)-\mathrm{H}(60 \mathrm{~B})$ & 0.9800 \\
\hline $\mathrm{C}(60)-\mathrm{H}(60 \mathrm{C})$ & 0.9800 \\
\hline $\mathrm{N}(1)-\mathrm{N}(2)$ & $1.340(16)$ \\
\hline $\mathrm{N}(3)-\mathrm{N}(4)$ & $1.365(18)$ \\
\hline
\end{tabular}




\begin{tabular}{|c|c|}
\hline$C(4)-S(1)-C(1)$ & $92.0(9)$ \\
\hline $\mathrm{C}(2)-\mathrm{C}(1)-\mathrm{S}(1)$ & 111.3(15) \\
\hline $\mathrm{C}(2)-\mathrm{C}(1)-\mathrm{H}(1)$ & 124.4 \\
\hline $\mathrm{S}(1)-\mathrm{C}(1)-\mathrm{H}(1)$ & 124.4 \\
\hline $\mathrm{C}(1)-\mathrm{C}(2)-\mathrm{C}(3)$ & $114.1(17)$ \\
\hline $\mathrm{C}(1)-\mathrm{C}(2)-\mathrm{H}(2)$ & 122.9 \\
\hline $\mathrm{C}(3)-\mathrm{C}(2)-\mathrm{H}(2)$ & 122.9 \\
\hline$C(4)-C(3)-C(2)$ & $111.4(14)$ \\
\hline$C(4)-C(3)-C(5)$ & $126.6(16)$ \\
\hline$C(2)-C(3)-C(5)$ & $122.1(16)$ \\
\hline $\mathrm{C}(3)-\mathrm{C}(4)-\mathrm{S}(1)$ & $111.2(13)$ \\
\hline $\mathrm{C}(3)-\mathrm{C}(4)-\mathrm{H}(4)$ & 124.4 \\
\hline $\mathrm{S}(1)-\mathrm{C}(4)-\mathrm{H}(4)$ & 124.4 \\
\hline$C\left(4^{\prime}\right)-S\left(1^{\prime}\right)-C\left(1^{\prime}\right)$ & $91(2)$ \\
\hline $\mathrm{C}\left(2^{\prime}\right)-\mathrm{C}\left(1^{\prime}\right)-\mathrm{S}\left(1^{\prime}\right)$ & $110(3)$ \\
\hline $\mathrm{C}\left(2^{\prime}\right)-\mathrm{C}\left(1^{\prime}\right)-\mathrm{H}\left(1^{\prime}\right)$ & 125.0 \\
\hline $\mathrm{S}\left(1^{\prime}\right)-\mathrm{C}\left(1^{\prime}\right)-\mathrm{H}\left(1^{\prime}\right)$ & 125.0 \\
\hline $\mathrm{C}\left(1^{\prime}\right)-\mathrm{C}\left(2^{\prime}\right)-\mathrm{C}\left(3^{\prime}\right)$ & $114(3)$ \\
\hline $\mathrm{C}\left(1^{\prime}\right)-\mathrm{C}\left(2^{\prime}\right)-\mathrm{H}\left(2^{\prime}\right)$ & 123.2 \\
\hline $\mathrm{C}\left(3^{\prime}\right)-\mathrm{C}\left(2^{\prime}\right)-\mathrm{H}\left(2^{\prime}\right)$ & 123.2 \\
\hline $\mathrm{C}\left(4^{\prime}\right)-\mathrm{C}\left(3^{\prime}\right)-\mathrm{C}\left(2^{\prime}\right)$ & 111(2) \\
\hline$C\left(4^{\prime}\right)-C\left(3^{\prime}\right)-C(5)$ & $137(10)$ \\
\hline$C\left(2^{\prime}\right)-C\left(3^{\prime}\right)-C(5)$ & $110(10)$ \\
\hline$C\left(3^{\prime}\right)-C\left(4^{\prime}\right)-S\left(1^{\prime}\right)$ & $110(3)$ \\
\hline $\mathrm{C}\left(3^{\prime}\right)-\mathrm{C}\left(4^{\prime}\right)-\mathrm{H}\left(4^{\prime}\right)$ & 124.9 \\
\hline $\mathrm{S}\left(1^{\prime}\right)-\mathrm{C}\left(4^{\prime}\right)-\mathrm{H}\left(4^{\prime}\right)$ & 124.8 \\
\hline$C(34)-S(2)-C(31)$ & $94.8(18)$ \\
\hline$C(32)-C(31)-S(2)$ & $108(3)$ \\
\hline $\mathrm{C}(32)-\mathrm{C}(31)-\mathrm{H}(31)$ & 125.9 \\
\hline $\mathrm{S}(2)-\mathrm{C}(31)-\mathrm{H}(31)$ & 125.9 \\
\hline$C(33)-C(32)-C(31)$ & 113(2) \\
\hline $\mathrm{C}(33)-\mathrm{C}(32)-\mathrm{C}(35)$ & $129(6)$ \\
\hline $\mathrm{C}(31)-\mathrm{C}(32)-\mathrm{C}(35)$ & $117(5)$ \\
\hline $\mathrm{C}(32)-\mathrm{C}(33)-\mathrm{C}(34)$ & $114(4)$ \\
\hline $\mathrm{C}(32)-\mathrm{C}(33)-\mathrm{H}(33)$ & 123.1 \\
\hline
\end{tabular}




\begin{tabular}{|c|c|}
\hline $\mathrm{C}(34)-\mathrm{C}(33)-\mathrm{H}(33)$ & 123.1 \\
\hline $\mathrm{C}(33)-\mathrm{C}(34)-\mathrm{S}(2)$ & $110(3)$ \\
\hline C(33)-C(34)-H(34) & 125.2 \\
\hline $\mathrm{S}(2)-\mathrm{C}(34)-\mathrm{H}(34)$ & 125.2 \\
\hline $\mathrm{C}\left(34^{\prime}\right)-\mathrm{S}\left(2^{\prime}\right)-\mathrm{C}\left(31^{\prime}\right)$ & $95.2(17)$ \\
\hline $\mathrm{C}\left(32^{\prime}\right)-\mathrm{C}\left(31^{\prime}\right)-\mathrm{S}\left(2^{\prime}\right)$ & $108(3)$ \\
\hline $\mathrm{C}\left(32^{\prime}\right)-\mathrm{C}\left(31^{\prime}\right)-\mathrm{H}\left(31^{\prime}\right)$ & 126.0 \\
\hline $\mathrm{S}\left(2^{\prime}\right)-\mathrm{C}\left(31^{\prime}\right)-\mathrm{H}\left(31^{\prime}\right)$ & 126.0 \\
\hline $\mathrm{C}\left(33^{\prime}\right)-\mathrm{C}\left(32^{\prime}\right)-\mathrm{C}(35)$ & $125(5)$ \\
\hline $\mathrm{C}\left(33^{\prime}\right)-\mathrm{C}\left(32^{\prime}\right)-\mathrm{C}\left(31^{\prime}\right)$ & $113(2)$ \\
\hline $\mathrm{C}(35)-\mathrm{C}\left(32^{\prime}\right)-\mathrm{C}\left(31^{\prime}\right)$ & $121(5)$ \\
\hline $\mathrm{C}\left(32^{\prime}\right)-\mathrm{C}\left(33^{\prime}\right)-\mathrm{C}\left(34^{\prime}\right)$ & $114(3)$ \\
\hline $\mathrm{C}\left(32^{\prime}\right)-\mathrm{C}\left(33^{\prime}\right)-\mathrm{H}\left(33^{\prime}\right)$ & 123.1 \\
\hline $\mathrm{C}\left(34^{\prime}\right)-\mathrm{C}\left(33^{\prime}\right)-\mathrm{H}\left(33^{\prime}\right)$ & 123.1 \\
\hline $\mathrm{C}\left(33^{\prime}\right)-\mathrm{C}\left(34^{\prime}\right)-\mathrm{S}\left(2^{\prime}\right)$ & $109(2)$ \\
\hline $\mathrm{C}\left(33^{\prime}\right)-\mathrm{C}\left(34^{\prime}\right)-\mathrm{H}\left(34^{\prime}\right)$ & 125.7 \\
\hline $\mathrm{S}\left(2^{\prime}\right)-\mathrm{C}\left(34^{\prime}\right)-\mathrm{H}\left(34^{\prime}\right)$ & 125.7 \\
\hline $\mathrm{N}(1)-\mathrm{C}(5)-\mathrm{C}\left(3^{\prime}\right)$ & $112(10)$ \\
\hline$N(1)-C(5)-C(6)$ & $110.8(11)$ \\
\hline$C\left(3^{\prime}\right)-C(5)-C(6)$ & $118(10)$ \\
\hline$N(1)-C(5)-C(3)$ & $111.5(13)$ \\
\hline$C(6)-C(5)-C(3)$ & $110.8(13)$ \\
\hline $\mathrm{N}(1)-\mathrm{C}(5)-\mathrm{H}(5)$ & 107.9 \\
\hline $\mathrm{C}(6)-\mathrm{C}(5)-\mathrm{H}(5)$ & 107.9 \\
\hline $\mathrm{C}(3)-\mathrm{C}(5)-\mathrm{H}(5)$ & 107.9 \\
\hline$C(7)-C(6)-C(9)$ & $106.3(13)$ \\
\hline$C(7)-C(6)-C(5)$ & $124.5(13)$ \\
\hline$C(9)-C(6)-C(5)$ & $129.1(13)$ \\
\hline $\mathrm{O}(1)-\mathrm{C}(7)-\mathrm{C}(6)$ & $111.0(13)$ \\
\hline $\mathrm{O}(1)-\mathrm{C}(7)-\mathrm{C}(8)$ & $115.9(13)$ \\
\hline$C(6)-C(7)-C(8)$ & $132.9(15)$ \\
\hline $\mathrm{C}(7)-\mathrm{C}(8)-\mathrm{H}(8 \mathrm{~A})$ & 109.5 \\
\hline $\mathrm{C}(7)-\mathrm{C}(8)-\mathrm{H}(8 \mathrm{~B})$ & 109.5 \\
\hline $\mathrm{H}(8 \mathrm{~A})-\mathrm{C}(8)-\mathrm{H}(8 \mathrm{~B})$ & 109.5 \\
\hline $\mathrm{C}(7)-\mathrm{C}(8)-\mathrm{H}(8 \mathrm{C})$ & 109.5 \\
\hline $\mathrm{H}(8 \mathrm{~A})-\mathrm{C}(8)-\mathrm{H}(8 \mathrm{C})$ & 109.5 \\
\hline
\end{tabular}




\begin{tabular}{|c|c|}
\hline $\mathrm{H}(8 \mathrm{~B})-\mathrm{C}(8)-\mathrm{H}(8 \mathrm{C})$ & 109.5 \\
\hline$C(10)-C(9)-C(6)$ & $108.2(13)$ \\
\hline $\mathrm{C}(10)-\mathrm{C}(9)-\mathrm{H}(9)$ & 125.9 \\
\hline $\mathrm{C}(6)-\mathrm{C}(9)-\mathrm{H}(9)$ & 125.9 \\
\hline $\mathrm{C}(9)-\mathrm{C}(10)-\mathrm{O}(1)$ & $107.4(12)$ \\
\hline $\mathrm{C}(9)-\mathrm{C}(10)-\mathrm{C}(11)$ & $135.0(13)$ \\
\hline $\mathrm{O}(1)-\mathrm{C}(10)-\mathrm{C}(11)$ & $117.3(12)$ \\
\hline $\mathrm{C}(12)-\mathrm{C}(11)-\mathrm{C}(16)$ & $116.9(14)$ \\
\hline $\mathrm{C}(12)-\mathrm{C}(11)-\mathrm{C}(10)$ & $120.9(13)$ \\
\hline$C(16)-C(11)-C(10)$ & $122.2(13)$ \\
\hline $\mathrm{C}(13)-\mathrm{C}(12)-\mathrm{C}(11)$ & $122.2(14)$ \\
\hline $\mathrm{C}(13)-\mathrm{C}(12)-\mathrm{H}(12)$ & 118.9 \\
\hline $\mathrm{C}(11)-\mathrm{C}(12)-\mathrm{H}(12)$ & 118.9 \\
\hline $\mathrm{C}(12)-\mathrm{C}(13)-\mathrm{C}(14)$ & $120.4(15)$ \\
\hline $\mathrm{C}(12)-\mathrm{C}(13)-\mathrm{H}(13)$ & 119.8 \\
\hline $\mathrm{C}(14)-\mathrm{C}(13)-\mathrm{H}(13)$ & 119.8 \\
\hline $\mathrm{C}(15)-\mathrm{C}(14)-\mathrm{C}(13)$ & $120.7(16)$ \\
\hline $\mathrm{C}(15)-\mathrm{C}(14)-\mathrm{Br}(1)$ & $117.5(12)$ \\
\hline$C(13)-C(14)-B r(1)$ & $121.8(12)$ \\
\hline $\mathrm{C}(14)-\mathrm{C}(15)-\mathrm{C}(16)$ & $117.2(15)$ \\
\hline $\mathrm{C}(14)-\mathrm{C}(15)-\mathrm{H}(15)$ & 121.4 \\
\hline $\mathrm{C}(16)-\mathrm{C}(15)-\mathrm{H}(15)$ & 121.4 \\
\hline $\mathrm{C}(11)-\mathrm{C}(16)-\mathrm{C}(15)$ & $122.6(14)$ \\
\hline $\mathrm{C}(11)-\mathrm{C}(16)-\mathrm{H}(16)$ & 118.7 \\
\hline $\mathrm{C}(15)-\mathrm{C}(16)-\mathrm{H}(16)$ & 118.7 \\
\hline $\mathrm{N}(1)-\mathrm{C}(17)-\mathrm{C}(18)$ & $108.9(13)$ \\
\hline $\mathrm{N}(1)-\mathrm{C}(17)-\mathrm{H}(17)$ & 125.6 \\
\hline $\mathrm{C}(18)-\mathrm{C}(17)-\mathrm{H}(17)$ & 125.6 \\
\hline $\mathrm{C}(17)-\mathrm{C}(18)-\mathrm{C}(19)$ & $104.6(13)$ \\
\hline $\mathrm{C}(17)-\mathrm{C}(18)-\mathrm{H}(18)$ & 127.7 \\
\hline $\mathrm{C}(19)-\mathrm{C}(18)-\mathrm{H}(18)$ & 127.7 \\
\hline $\mathrm{N}(2)-\mathrm{C}(19)-\mathrm{C}(18)$ & $109.7(12)$ \\
\hline $\mathrm{N}(2)-\mathrm{C}(19)-\mathrm{C}(20)$ & $121.3(12)$ \\
\hline $\mathrm{C}(18)-\mathrm{C}(19)-\mathrm{C}(20)$ & $129.0(13)$ \\
\hline $\mathrm{O}(2)-\mathrm{C}(20)-\mathrm{C}(19)$ & $117.6(13)$ \\
\hline $\mathrm{O}(2)-\mathrm{C}(20)-\mathrm{C}(21)$ & $120.5(12)$ \\
\hline
\end{tabular}




\begin{tabular}{|c|c|}
\hline$C(19)-C(20)-C(21)$ & $121.8(12)$ \\
\hline$C(30)-C(21)-C(22)$ & $117.7(12)$ \\
\hline$C(30)-C(21)-C(20)$ & $123.6(12)$ \\
\hline$C(22)-C(21)-C(20)$ & $118.6(12)$ \\
\hline$C(23)-C(22)-C(21)$ & $122.3(13)$ \\
\hline $\mathrm{C}(23)-\mathrm{C}(22)-\mathrm{H}(22)$ & 118.9 \\
\hline $\mathrm{C}(21)-\mathrm{C}(22)-\mathrm{H}(22)$ & 118.9 \\
\hline $\mathrm{C}(22)-\mathrm{C}(23)-\mathrm{C}(24)$ & $120.2(13)$ \\
\hline $\mathrm{C}(22)-\mathrm{C}(23)-\mathrm{H}(23)$ & 119.9 \\
\hline $\mathrm{C}(24)-\mathrm{C}(23)-\mathrm{H}(23)$ & 119.9 \\
\hline$C(23)-C(24)-C(29)$ & $119.5(13)$ \\
\hline$C(23)-C(24)-C(25)$ & $122.3(13)$ \\
\hline$C(29)-C(24)-C(25)$ & 118.1(13) \\
\hline$C(26)-C(25)-C(24)$ & $120.1(14)$ \\
\hline $\mathrm{C}(26)-\mathrm{C}(25)-\mathrm{H}(25)$ & 120.0 \\
\hline $\mathrm{C}(24)-\mathrm{C}(25)-\mathrm{H}(25)$ & 120.0 \\
\hline$C(25)-C(26)-C(27)$ & $122.5(15)$ \\
\hline $\mathrm{C}(25)-\mathrm{C}(26)-\mathrm{H}(26)$ & 118.7 \\
\hline $\mathrm{C}(27)-\mathrm{C}(26)-\mathrm{H}(26)$ & 118.7 \\
\hline$C(28)-C(27)-C(26)$ & $119.0(14)$ \\
\hline $\mathrm{C}(28)-\mathrm{C}(27)-\mathrm{H}(27)$ & 120.5 \\
\hline $\mathrm{C}(26)-\mathrm{C}(27)-\mathrm{H}(27)$ & 120.5 \\
\hline$C(27)-C(28)-C(29)$ & $120.4(14)$ \\
\hline $\mathrm{C}(27)-\mathrm{C}(28)-\mathrm{H}(28)$ & 119.8 \\
\hline $\mathrm{C}(29)-\mathrm{C}(28)-\mathrm{H}(28)$ & 119.8 \\
\hline $\mathrm{C}(30)-\mathrm{C}(29)-\mathrm{C}(28)$ & $121.1(13)$ \\
\hline$C(30)-C(29)-C(24)$ & $119.0(12)$ \\
\hline $\mathrm{C}(28)-\mathrm{C}(29)-\mathrm{C}(24)$ & $119.9(13)$ \\
\hline$C(29)-C(30)-C(21)$ & $121.3(12)$ \\
\hline $\mathrm{C}(29)-\mathrm{C}(30)-\mathrm{H}(30)$ & 119.3 \\
\hline $\mathrm{C}(21)-\mathrm{C}(30)-\mathrm{H}(30)$ & 119.3 \\
\hline $\mathrm{C}\left(32^{\prime}\right)-\mathrm{C}(35)-\mathrm{N}(3)$ & $113(3)$ \\
\hline $\mathrm{C}\left(32^{\prime}\right)-\mathrm{C}(35)-\mathrm{C}(51)$ & $112(3)$ \\
\hline$N(3)-C(35)-C(51)$ & $110.2(13)$ \\
\hline $\mathrm{N}(3)-\mathrm{C}(35)-\mathrm{C}(32)$ & $113(4)$ \\
\hline $\mathrm{C}(51)-\mathrm{C}(35)-\mathrm{C}(32)$ & $113(3)$ \\
\hline
\end{tabular}




\begin{tabular}{|c|c|}
\hline $\mathrm{N}(3)-\mathrm{C}(35)-\mathrm{H}(35)$ & 106.7 \\
\hline $\mathrm{C}(51)-\mathrm{C}(35)-\mathrm{H}(35)$ & 106.7 \\
\hline $\mathrm{C}(32)-\mathrm{C}(35)-\mathrm{H}(35)$ & 106.7 \\
\hline $\mathrm{C}(37)-\mathrm{C}(36)-\mathrm{N}(3)$ & $106.4(15)$ \\
\hline $\mathrm{C}(37)-\mathrm{C}(36)-\mathrm{H}(36)$ & 126.8 \\
\hline $\mathrm{N}(3)-\mathrm{C}(36)-\mathrm{H}(36)$ & 126.8 \\
\hline $\mathrm{C}(36)-\mathrm{C}(37)-\mathrm{C}(38)$ & $105.6(14)$ \\
\hline $\mathrm{C}(36)-\mathrm{C}(37)-\mathrm{H}(37)$ & 127.2 \\
\hline $\mathrm{C}(38)-\mathrm{C}(37)-\mathrm{H}(37)$ & 127.2 \\
\hline $\mathrm{N}(4)-\mathrm{C}(38)-\mathrm{C}(37)$ & $112.4(15)$ \\
\hline $\mathrm{N}(4)-\mathrm{C}(38)-\mathrm{C}(39)$ & $122.0(15)$ \\
\hline C(37)-C(38)-C(39) & $125.6(15)$ \\
\hline $\mathrm{O}(4)-\mathrm{C}(39)-\mathrm{C}(38)$ & $119.3(16)$ \\
\hline $\mathrm{O}(4)-\mathrm{C}(39)-\mathrm{C}(40)$ & $119.6(15)$ \\
\hline $\mathrm{C}(38)-\mathrm{C}(39)-\mathrm{C}(40)$ & $121.1(13)$ \\
\hline$C(49)-C(40)-C(41)$ & $119.2(15)$ \\
\hline C(49)-C(40)-C(39) & $123.5(15)$ \\
\hline $\mathrm{C}(41)-\mathrm{C}(40)-\mathrm{C}(39)$ & $117.3(14)$ \\
\hline$C(42)-C(41)-C(40)$ & $120.4(15)$ \\
\hline $\mathrm{C}(42)-\mathrm{C}(41)-\mathrm{H}(41)$ & 119.8 \\
\hline $\mathrm{C}(40)-\mathrm{C}(41)-\mathrm{H}(41)$ & 119.8 \\
\hline C(41)-C(42)-C(43) & $121.1(16)$ \\
\hline $\mathrm{C}(41)-\mathrm{C}(42)-\mathrm{H}(42)$ & 119.4 \\
\hline $\mathrm{C}(43)-\mathrm{C}(42)-\mathrm{H}(42)$ & 119.4 \\
\hline C(44)-C(43)-C(42) & $122.3(16)$ \\
\hline$C(44)-C(43)-C(48)$ & $120.0(15)$ \\
\hline$C(42)-C(43)-C(48)$ & $117.7(15)$ \\
\hline $\mathrm{C}(45)-\mathrm{C}(44)-\mathrm{C}(43)$ & $120.1(16)$ \\
\hline $\mathrm{C}(45)-\mathrm{C}(44)-\mathrm{H}(44)$ & 119.9 \\
\hline $\mathrm{C}(43)-\mathrm{C}(44)-\mathrm{H}(44)$ & 119.9 \\
\hline$C(44)-C(45)-C(46)$ & $121.5(17)$ \\
\hline $\mathrm{C}(44)-\mathrm{C}(45)-\mathrm{H}(45)$ & 119.2 \\
\hline $\mathrm{C}(46)-\mathrm{C}(45)-\mathrm{H}(45)$ & 119.2 \\
\hline$C(47)-C(46)-C(45)$ & $118.2(18)$ \\
\hline $\mathrm{C}(47)-\mathrm{C}(46)-\mathrm{H}(46)$ & 120.9 \\
\hline $\mathrm{C}(45)-\mathrm{C}(46)-\mathrm{H}(46)$ & 120.9 \\
\hline
\end{tabular}




\begin{tabular}{|c|c|}
\hline $\mathrm{C}(46)-\mathrm{C}(47)-\mathrm{C}(48)$ & $123.2(17)$ \\
\hline $\mathrm{C}(46)-\mathrm{C}(47)-\mathrm{H}(47)$ & 118.4 \\
\hline $\mathrm{C}(48)-\mathrm{C}(47)-\mathrm{H}(47)$ & 118.4 \\
\hline $\mathrm{C}(49)-\mathrm{C}(48)-\mathrm{C}(47)$ & $123.6(15)$ \\
\hline $\mathrm{C}(49)-\mathrm{C}(48)-\mathrm{C}(43)$ & $119.4(15)$ \\
\hline $\mathrm{C}(47)-\mathrm{C}(48)-\mathrm{C}(43)$ & $116.8(15)$ \\
\hline $\mathrm{C}(40)-\mathrm{C}(49)-\mathrm{C}(48)$ & $122.1(15)$ \\
\hline $\mathrm{C}(40)-\mathrm{C}(49)-\mathrm{H}(49)$ & 118.9 \\
\hline $\mathrm{C}(48)-\mathrm{C}(49)-\mathrm{H}(49)$ & 118.9 \\
\hline $\mathrm{O}(3)-\mathrm{C}(50)-\mathrm{C}(51)$ & $108.8(12)$ \\
\hline $\mathrm{O}(3)-\mathrm{C}(50)-\mathrm{C}(60)$ & $118.6(13)$ \\
\hline$C(51)-C(50)-C(60)$ & $132.6(14)$ \\
\hline $\mathrm{C}(50)-\mathrm{C}(51)-\mathrm{C}(52)$ & $107.1(13)$ \\
\hline $\mathrm{C}(50)-\mathrm{C}(51)-\mathrm{C}(35)$ & $125.4(14)$ \\
\hline$C(52)-C(51)-C(35)$ & $127.2(13)$ \\
\hline $\mathrm{C}(53)-\mathrm{C}(52)-\mathrm{C}(51)$ & $105.9(13)$ \\
\hline $\mathrm{C}(53)-\mathrm{C}(52)-\mathrm{H}(52)$ & 127.0 \\
\hline $\mathrm{C}(51)-\mathrm{C}(52)-\mathrm{H}(52)$ & 127.0 \\
\hline $\mathrm{C}(52)-\mathrm{C}(53)-\mathrm{O}(3)$ & $109.9(12)$ \\
\hline $\mathrm{C}(52)-\mathrm{C}(53)-\mathrm{C}(54)$ & $133.0(14)$ \\
\hline $\mathrm{O}(3)-\mathrm{C}(53)-\mathrm{C}(54)$ & $117.1(12)$ \\
\hline$C(55)-C(54)-C(59)$ & $116.7(13)$ \\
\hline $\mathrm{C}(55)-\mathrm{C}(54)-\mathrm{C}(53)$ & $121.4(13)$ \\
\hline$C(59)-C(54)-C(53)$ & $121.8(14)$ \\
\hline$C(56)-C(55)-C(54)$ & $121.1(15)$ \\
\hline $\mathrm{C}(56)-\mathrm{C}(55)-\mathrm{H}(55)$ & 119.4 \\
\hline $\mathrm{C}(54)-\mathrm{C}(55)-\mathrm{H}(55)$ & 119.4 \\
\hline$C(55)-C(56)-C(57)$ & $120.3(15)$ \\
\hline $\mathrm{C}(55)-\mathrm{C}(56)-\mathrm{H}(56)$ & 119.8 \\
\hline $\mathrm{C}(57)-\mathrm{C}(56)-\mathrm{H}(56)$ & 119.8 \\
\hline$C(56)-C(57)-C(58)$ & $120.2(15)$ \\
\hline $\mathrm{C}(56)-\mathrm{C}(57)-\mathrm{Br}(2)$ & $120.3(12)$ \\
\hline $\mathrm{C}(58)-\mathrm{C}(57)-\mathrm{Br}(2)$ & $119.4(12)$ \\
\hline$C(59)-C(58)-C(57)$ & $118.6(15)$ \\
\hline $\mathrm{C}(59)-\mathrm{C}(58)-\mathrm{H}(58)$ & 120.7 \\
\hline $\mathrm{C}(57)-\mathrm{C}(58)-\mathrm{H}(58)$ & 120.7 \\
\hline
\end{tabular}




$\begin{array}{ll}\mathrm{C}(58)-\mathrm{C}(59)-\mathrm{C}(54) & 122.9(15) \\ \mathrm{C}(58)-\mathrm{C}(59)-\mathrm{H}(59) & 118.5 \\ \mathrm{C}(54)-\mathrm{C}(59)-\mathrm{H}(59) & 118.5 \\ \mathrm{C}(50)-\mathrm{C}(60)-\mathrm{H}(60 \mathrm{~A}) & 109.5 \\ \mathrm{C}(50)-\mathrm{C}(60)-\mathrm{H}(60 \mathrm{~B}) & 109.5 \\ \mathrm{H}(60 \mathrm{~A})-\mathrm{C}(60)-\mathrm{H}(60 \mathrm{~B}) & 109.5 \\ \mathrm{C}(50)-\mathrm{C}(60)-\mathrm{H}(60 \mathrm{C}) & 109.5 \\ \mathrm{H}(60 \mathrm{~A})-\mathrm{C}(60)-\mathrm{H}(60 \mathrm{C}) & 109.5 \\ \mathrm{H}(60 \mathrm{~B})-\mathrm{C}(60)-\mathrm{H}(60 \mathrm{C}) & 109.5 \\ \mathrm{C}(17)-\mathrm{N}(1)-\mathrm{N}(2) & 112.4(12) \\ \mathrm{C}(17)-\mathrm{N}(1)-\mathrm{C}(5) & 126.7(12) \\ \mathrm{N}(2)-\mathrm{N}(1)-\mathrm{C}(5) & 120.9(12) \\ \mathrm{N}(1)-\mathrm{N}(2)-\mathrm{C}(19) & 104.3(11) \\ \mathrm{N}(4)-\mathrm{N}(3)-\mathrm{C}(36) & 112.7(14) \\ \mathrm{N}(4)-\mathrm{N}(3)-\mathrm{C}(35) & 121.0(12) \\ \mathrm{C}(36)-\mathrm{N}(3)-\mathrm{C}(35) & 126.4(14) \\ \mathrm{C}(38)-\mathrm{N}(4)-\mathrm{N}(3) & 102.9(13) \\ \mathrm{C}(7)-\mathrm{O}(1)-\mathrm{C}(10) & 107.0(11) \\ \mathrm{C}(50)-\mathrm{O}(3)-\mathrm{C}(53) & 108.2(11)\end{array}$

Symmetry transformations used to generate equivalent atoms: 
Table S9. Anisotropic displacement parameters $\left(\AA^{2} \times 10^{3}\right)$ for twin5. The anisotropic displacement factor exponent takes the form: $-2 \pi^{2}\left[h^{2} a^{* 2} U^{11}+\ldots+2 h k a^{*} b^{*} U^{12}\right]$

\begin{tabular}{|c|c|c|c|c|c|c|}
\hline & $\mathrm{U}^{11}$ & $\mathrm{U}^{22}$ & $\mathrm{U}^{33}$ & $\mathrm{U}^{23}$ & $\mathrm{U}^{13}$ & $\mathrm{U}^{12}$ \\
\hline $\operatorname{Br}(1)$ & $45(1)$ & $41(1)$ & 21(1) & $0(1)$ & $-3(1)$ & $-21(1)$ \\
\hline $\operatorname{Br}(2)$ & $58(1)$ & $55(1)$ & $16(1)$ & $-8(1)$ & $6(1)$ & $-28(1)$ \\
\hline$S(1)$ & $35(2)$ & $43(3)$ & $19(2)$ & $-6(2)$ & $2(2)$ & $-28(2)$ \\
\hline $\mathrm{C}(1)$ & $35(4)$ & $39(4)$ & $16(5)$ & $-6(4)$ & 4(4) & $-24(4)$ \\
\hline$C(2)$ & $35(4)$ & $32(4)$ & $19(5)$ & $-5(4)$ & $3(4)$ & $-20(3)$ \\
\hline$C(3)$ & $32(4)$ & $32(4)$ & $17(5)$ & $-4(4)$ & $3(4)$ & $-20(3)$ \\
\hline$C(4)$ & $31(4)$ & $37(4)$ & $18(5)$ & $-7(4)$ & $3(4)$ & $-21(3)$ \\
\hline$S\left(1^{\prime}\right)$ & $34(5)$ & $38(5)$ & $18(5)$ & $-5(4)$ & $3(5)$ & $-23(4)$ \\
\hline$C\left(1^{\prime}\right)$ & $33(5)$ & $38(5)$ & $19(6)$ & $-6(5)$ & $3(5)$ & $-23(4)$ \\
\hline $\mathrm{C}\left(2^{\prime}\right)$ & $32(4)$ & $37(5)$ & $19(5)$ & $-6(4)$ & $3(4)$ & $-22(4)$ \\
\hline$C\left(3^{\prime}\right)$ & $32(4)$ & $34(5)$ & $19(5)$ & $-5(4)$ & $4(4)$ & $-21(4)$ \\
\hline$C\left(4^{\prime}\right)$ & $34(5)$ & $35(5)$ & $18(5)$ & $-5(4)$ & $4(5)$ & $-22(4)$ \\
\hline$S(2)$ & $39(4)$ & $63(5)$ & $15(5)$ & $5(3)$ & $0(3)$ & $-26(4)$ \\
\hline $\mathrm{C}(31)$ & $38(5)$ & $60(5)$ & $12(6)$ & 1(4) & $0(4)$ & $-26(4)$ \\
\hline$C(32)$ & $34(5)$ & $60(5)$ & $10(6)$ & $0(4)$ & $-2(4)$ & $-27(4)$ \\
\hline $\mathrm{C}(33)$ & $35(5)$ & $61(6)$ & $11(6)$ & 1(4) & $-2(4)$ & $-28(4)$ \\
\hline$C(34)$ & $37(5)$ & $61(6)$ & $15(6)$ & $2(5)$ & $-1(4)$ & $-29(4)$ \\
\hline$S\left(2^{\prime}\right)$ & $38(4)$ & $65(5)$ & $17(4)$ & $3(3)$ & $-1(3)$ & $-33(4)$ \\
\hline $\mathrm{C}\left(31^{\prime}\right)$ & $34(5)$ & $62(5)$ & $15(6)$ & $2(4)$ & $-1(4)$ & $-28(4)$ \\
\hline$C\left(32^{\prime}\right)$ & $34(5)$ & $59(5)$ & $9(5)$ & $-1(4)$ & $-1(4)$ & $-27(4)$ \\
\hline$C\left(33^{\prime}\right)$ & $37(5)$ & $60(5)$ & $12(6)$ & $1(4)$ & $0(4)$ & $-27(4)$ \\
\hline$C\left(34^{\prime}\right)$ & $39(5)$ & $63(5)$ & $16(6)$ & $2(4)$ & $-1(4)$ & $-29(4)$ \\
\hline$C(5)$ & $18(5)$ & $21(4)$ & $20(5)$ & $-6(3)$ & $7(3)$ & $-9(4)$ \\
\hline$C(6)$ & $22(5)$ & $15(6)$ & $23(4)$ & $-4(3)$ & $3(3)$ & $-8(4)$ \\
\hline$C(7)$ & $20(5)$ & $13(6)$ & $25(5)$ & $-6(4)$ & $3(3)$ & $-4(4)$ \\
\hline $\mathrm{C}(8)$ & $25(6)$ & $29(8)$ & $23(7)$ & $-8(6)$ & $2(5)$ & $-17(6)$ \\
\hline $\mathrm{C}(9)$ & $14(5)$ & $15(6)$ & $22(4)$ & $-3(4)$ & $0(4)$ & $-4(4)$ \\
\hline$C(10)$ & $12(5)$ & $17(6)$ & $22(4)$ & $-3(4)$ & $0(3)$ & $-3(4)$ \\
\hline $\mathrm{C}(11)$ & $18(5)$ & $17(6)$ & $21(4)$ & $-4(4)$ & $-1(3)$ & $-7(5)$ \\
\hline$C(12)$ & $18(5)$ & $31(8)$ & $21(5)$ & $-1(5)$ & $-4(4)$ & $-13(5)$ \\
\hline \multirow[t]{2}{*}{$C(13)$} & $24(6)$ & $28(8)$ & $20(5)$ & $-2(5)$ & $0(4)$ & $-9(5)$ \\
\hline & & & & S50 & & \\
\hline
\end{tabular}




\begin{tabular}{|c|c|c|c|c|c|c|}
\hline$C(14)$ & $25(5)$ & $12(6)$ & $26(5)$ & $-4(4)$ & $-1(4)$ & $-5(5)$ \\
\hline$C(15)$ & $26(6)$ & $26(7)$ & $23(5)$ & $-5(5)$ & $0(4)$ & $-5(5)$ \\
\hline$C(16)$ & $13(5)$ & $19(7)$ & $22(5)$ & $-7(4)$ & $-2(4)$ & $-3(5)$ \\
\hline $\mathrm{C}(17)$ & $31(6)$ & $25(5)$ & $17(5)$ & $-5(4)$ & $10(5)$ & $-13(5)$ \\
\hline $\mathrm{C}(18)$ & $26(6)$ & $24(5)$ & $19(5)$ & $-8(4)$ & $6(4)$ & $-14(4)$ \\
\hline $\mathrm{C}(19)$ & $18(5)$ & $22(4)$ & $14(5)$ & $-4(3)$ & $1(4)$ & $-10(4)$ \\
\hline$C(20)$ & $16(5)$ & $22(4)$ & $16(4)$ & $-3(3)$ & $-3(4)$ & $-10(4)$ \\
\hline $\mathrm{C}(21)$ & $15(4)$ & $16(5)$ & $17(4)$ & $-4(3)$ & $-1(3)$ & $-7(4)$ \\
\hline$C(22)$ & $14(4)$ & $14(5)$ & $12(4)$ & $-6(4)$ & $-5(3)$ & $-8(4)$ \\
\hline$C(23)$ & $19(5)$ & $21(6)$ & $18(4)$ & $-7(4)$ & $-1(4)$ & $-8(5)$ \\
\hline$C(24)$ & $19(5)$ & $20(5)$ & $15(4)$ & $-4(4)$ & $-1(3)$ & $-9(4)$ \\
\hline$C(25)$ & $22(5)$ & $23(6)$ & $18(4)$ & $-4(4)$ & $0(4)$ & $-13(5)$ \\
\hline$C(26)$ & $22(5)$ & $32(6)$ & $23(5)$ & $1(5)$ & $0(4)$ & $-17(5)$ \\
\hline$C(27)$ & 21(5) & $34(7)$ & $22(5)$ & $6(5)$ & $-1(4)$ & $-10(5)$ \\
\hline$C(28)$ & $20(5)$ & $26(6)$ & $19(4)$ & $2(4)$ & $-2(4)$ & $-12(5)$ \\
\hline C(29) & $18(5)$ & $20(5)$ & $16(4)$ & $-3(4)$ & $-1(3)$ & $-9(4)$ \\
\hline $\mathrm{C}(30)$ & $9(5)$ & $17(5)$ & $16(4)$ & $-2(3)$ & $-3(3)$ & $-8(4)$ \\
\hline$C(35)$ & $20(5)$ & $45(5)$ & $16(5)$ & $-2(4)$ & $3(4)$ & $-12(4)$ \\
\hline$C(36)$ & $34(7)$ & $61(7)$ & $27(6)$ & $-18(5)$ & $-7(6)$ & $-8(6)$ \\
\hline$C(37)$ & $40(7)$ & $58(7)$ & $18(6)$ & $-22(5)$ & $-3(6)$ & $-9(6)$ \\
\hline$C(38)$ & $36(6)$ & $43(5)$ & $24(6)$ & $-20(4)$ & $-1(5)$ & $-7(5)$ \\
\hline C(39) & $28(6)$ & $40(6)$ & $33(5)$ & $-20(4)$ & $4(4)$ & $-9(5)$ \\
\hline $\mathrm{C}(40)$ & $29(5)$ & $24(6)$ & $33(5)$ & $-10(4)$ & $4(4)$ & $-14(5)$ \\
\hline $\mathrm{C}(41)$ & $27(5)$ & $24(7)$ & $37(6)$ & $-11(5)$ & $6(4)$ & $-14(5)$ \\
\hline $\mathrm{C}(42)$ & $29(6)$ & $25(7)$ & $37(6)$ & $-7(5)$ & $2(4)$ & $-12(5)$ \\
\hline $\mathrm{C}(43)$ & $28(5)$ & $21(6)$ & $38(5)$ & $-5(4)$ & $3(4)$ & $-13(5)$ \\
\hline $\mathrm{C}(44)$ & $32(6)$ & $30(7)$ & $38(6)$ & $-5(5)$ & $3(4)$ & $-14(6)$ \\
\hline$C(45)$ & $31(6)$ & $41(8)$ & $31(6)$ & $-3(5)$ & $2(5)$ & $-14(6)$ \\
\hline $\mathrm{C}(46)$ & $35(6)$ & $61(10)$ & $35(7)$ & $-7(6)$ & $2(5)$ & $-10(6)$ \\
\hline $\mathrm{C}(47)$ & $30(6)$ & $39(8)$ & $34(6)$ & $-4(5)$ & $2(4)$ & $-12(6)$ \\
\hline $\mathrm{C}(48)$ & $26(5)$ & $26(6)$ & $34(5)$ & $-2(4)$ & $4(4)$ & $-17(5)$ \\
\hline C(49) & $24(6)$ & $25(7)$ & $35(5)$ & $-10(4)$ & $6(4)$ & $-9(5)$ \\
\hline$C(50)$ & $20(5)$ & $26(7)$ & $18(5)$ & $1(4)$ & $4(3)$ & $-11(5)$ \\
\hline$C(51)$ & $21(5)$ & $25(7)$ & $17(4)$ & $-2(4)$ & $3(3)$ & $-9(5)$ \\
\hline$C(52)$ & $25(5)$ & $29(8)$ & $16(4)$ & $-2(4)$ & $4(4)$ & $-15(5)$ \\
\hline$C(53)$ & $19(5)$ & $19(7)$ & $17(4)$ & $-2(4)$ & $4(3)$ & $-5(5)$ \\
\hline
\end{tabular}




\begin{tabular}{lllllll}
$\mathrm{C}(54)$ & $23(5)$ & $20(7)$ & $15(4)$ & $-1(4)$ & $5(3)$ & $-8(5)$ \\
$\mathrm{C}(55)$ & $26(6)$ & $28(8)$ & $20(5)$ & $-7(5)$ & $3(4)$ & $-10(5)$ \\
$\mathrm{C}(56)$ & $36(6)$ & $39(9)$ & $18(5)$ & $-8(5)$ & $2(4)$ & $-21(6)$ \\
$\mathrm{C}(57)$ & $37(6)$ & $17(7)$ & $23(5)$ & $-2(4)$ & $5(4)$ & $-12(6)$ \\
$\mathrm{C}(58)$ & $40(6)$ & $40(9)$ & $14(5)$ & $-3(5)$ & $10(4)$ & $-21(6)$ \\
$\mathrm{C}(59)$ & $26(6)$ & $25(8)$ & $16(5)$ & $-1(4)$ & $8(4)$ & $-9(5)$ \\
$\mathrm{C}(60)$ & $20(6)$ & $36(9)$ & $30(7)$ & $-4(6)$ & $2(5)$ & $-13(6)$ \\
$\mathrm{N}(1)$ & $24(5)$ & $23(4)$ & $12(4)$ & $-7(3)$ & $10(4)$ & $-11(3)$ \\
$\mathrm{N}(2)$ & $17(5)$ & $22(4)$ & $16(5)$ & $-7(3)$ & $4(4)$ & $-10(4)$ \\
$\mathrm{N}(3)$ & $17(5)$ & $46(4)$ & $19(5)$ & $-9(4)$ & $-1(4)$ & $-10(4)$ \\
$\mathrm{N}(4)$ & $22(5)$ & $41(5)$ & $21(5)$ & $-16(4)$ & $1(4)$ & $-3(4)$ \\
$\mathrm{O}(1)$ & $11(4)$ & $13(5)$ & $26(4)$ & $-6(3)$ & $0(3)$ & $-2(4)$ \\
$\mathrm{O}(2)$ & $31(5)$ & $20(4)$ & $11(4)$ & $-5(3)$ & $4(4)$ & $-10(4)$ \\
$\mathrm{O}(3)$ & $22(4)$ & $24(5)$ & $17(4)$ & $3(3)$ & $4(3)$ & $-10(4)$ \\
$\mathrm{O}(4)$ & $50(8)$ & $50(6)$ & $43(6)$ & $-31(5)$ & $8(6)$ & $-10(6)$ \\
& & & & & & \\
\hline
\end{tabular}


Table S10. Hydrogen coordinates ( $\left.\times 10^{4}\right)$ and isotropic displacement parameters $\left(\AA^{2} \times 10^{3}\right)$ for twin5.

\begin{tabular}{|c|c|c|c|c|}
\hline & $\mathrm{x}$ & $\mathrm{y}$ & $\mathrm{z}$ & $\mathrm{U}(\mathrm{eq})$ \\
\hline $\mathrm{H}(1)$ & 7091 & -3777 & 7999 & 33 \\
\hline $\mathrm{H}(2)$ & 9073 & -3103 & 8167 & 32 \\
\hline $\mathrm{H}(4)$ & 5230 & 693 & 8135 & 31 \\
\hline $\mathrm{H}\left(1^{\prime}\right)$ & 4024 & -521 & 8121 & 33 \\
\hline $\mathrm{H}\left(2^{\prime}\right)$ & 5539 & 542 & 8424 & 32 \\
\hline $\mathrm{H}\left(4^{\prime}\right)$ & 8892 & -2834 & 7773 & 32 \\
\hline $\mathrm{H}(31)$ & 4653 & 891 & 7034 & 42 \\
\hline $\mathrm{H}(33)$ & 441 & 4129 & 6623 & 40 \\
\hline $\mathrm{H}(34)$ & -302 & 2123 & 6766 & 42 \\
\hline $\mathrm{H}\left(31^{\prime}\right)$ & 420 & 4633 & 6787 & 41 \\
\hline $\mathrm{H}\left(33^{\prime}\right)$ & 4446 & 994 & 6927 & 41 \\
\hline $\mathrm{H}\left(34^{\prime}\right)$ & 2379 & 80 & 6945 & 45 \\
\hline $\mathrm{H}(5)$ & 9487 & -781 & 8140 & 24 \\
\hline $\mathrm{H}(8 \mathrm{~A})$ & 12285 & -525 & 9009 & 35 \\
\hline $\mathrm{H}(8 \mathrm{~B})$ & 11662 & -646 & 8483 & 35 \\
\hline $\mathrm{H}(8 \mathrm{C})$ & 12684 & -2027 & 8817 & 35 \\
\hline $\mathrm{H}(9)$ & 6998 & -634 & 9265 & 21 \\
\hline $\mathrm{H}(12)$ & 6549 & -1185 & 10145 & 27 \\
\hline $\mathrm{H}(13)$ & 6260 & -1897 & 10931 & 30 \\
\hline $\mathrm{H}(15)$ & 10712 & -2652 & 11260 & 32 \\
\hline $\mathrm{H}(16)$ & 10993 & -1969 & 10437 & 23 \\
\hline $\mathrm{H}(17)$ & 8861 & 1378 & 7584 & 29 \\
\hline $\mathrm{H}(18)$ & 7245 & 3929 & 7637 & 26 \\
\hline $\mathrm{H}(22)$ & 2087 & 5998 & 8694 & 15 \\
\hline $\mathrm{H}(23)$ & 730 & 6041 & 9400 & 23 \\
\hline $\mathrm{H}(25)$ & 733 & 5321 & 10304 & 24 \\
\hline $\mathrm{H}(26)$ & 2056 & 3945 & 10964 & 29 \\
\hline $\mathrm{H}(27)$ & 4766 & 2452 & 10934 & 32 \\
\hline $\mathrm{H}(28)$ & 6149 & 2411 & 10209 & 25 \\
\hline $\mathrm{H}(30)$ & 6143 & 3141 & 9340 & 16 \\
\hline
\end{tabular}




\begin{tabular}{|c|c|c|c|c|}
\hline $\mathrm{H}(35)$ & 4672 & 3203 & 6862 & 33 \\
\hline $\mathrm{H}(36)$ & 4096 & 5075 & 7474 & 53 \\
\hline $\mathrm{H}(37)$ & 2217 & 7607 & 7492 & 50 \\
\hline $\mathrm{H}(41)$ & -2950 & 10207 & 6466 & 34 \\
\hline $\mathrm{H}(42)$ & -4243 & 10729 & 5722 & 36 \\
\hline $\mathrm{H}(44)$ & -4171 & 10533 & 4822 & 40 \\
\hline $\mathrm{H}(45)$ & -2782 & 9519 & 4127 & 42 \\
\hline $\mathrm{H}(46)$ & -99 & 7928 & 4177 & 57 \\
\hline $\mathrm{H}(47)$ & 1184 & 7494 & 4921 & 42 \\
\hline $\mathrm{H}(49)$ & 1113 & 7726 & 5819 & 34 \\
\hline $\mathrm{H}(52)$ & 2049 & 3946 & 5733 & 27 \\
\hline $\mathrm{H}(55)$ & 1510 & 3888 & 4850 & 30 \\
\hline $\mathrm{H}(56)$ & 1132 & 3760 & 4023 & 35 \\
\hline $\mathrm{H}(58)$ & 5680 & 2968 & 3746 & 36 \\
\hline $\mathrm{H}(59)$ & 6000 & 3220 & 4555 & 28 \\
\hline $\mathrm{H}(60 \mathrm{~A})$ & 7739 & 2299 & 6196 & 42 \\
\hline $\mathrm{H}(60 \mathrm{~B})$ & 6761 & 3576 & 6529 & 42 \\
\hline $\mathrm{H}(60 \mathrm{C})$ & 7421 & 3852 & 6008 & 42 \\
\hline
\end{tabular}

\section{6. ${ }^{1} \mathrm{H}$ and ${ }^{13} \mathrm{C}$ spectra of new compounds:}


Solvent: $\mathrm{CDCl}_{3}$

SFO1: $400 \mathrm{MHz}$

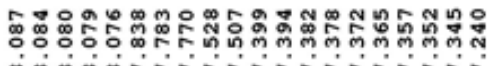

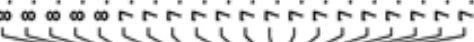

RDK-03-19

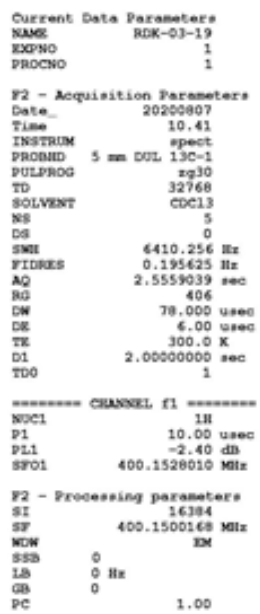

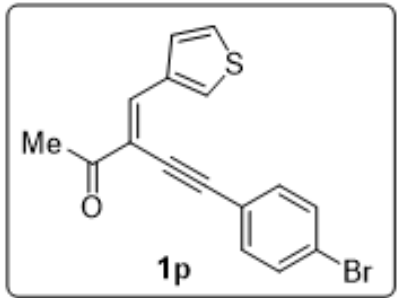

(n)

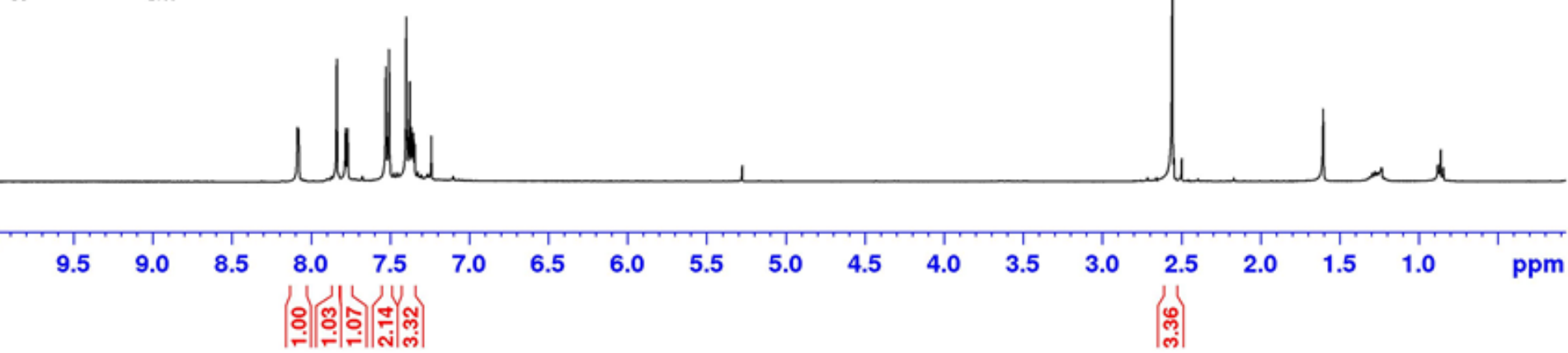


Solvent: $\mathrm{CDCl}_{3}$

SFO1: $100 \mathrm{MHz}$
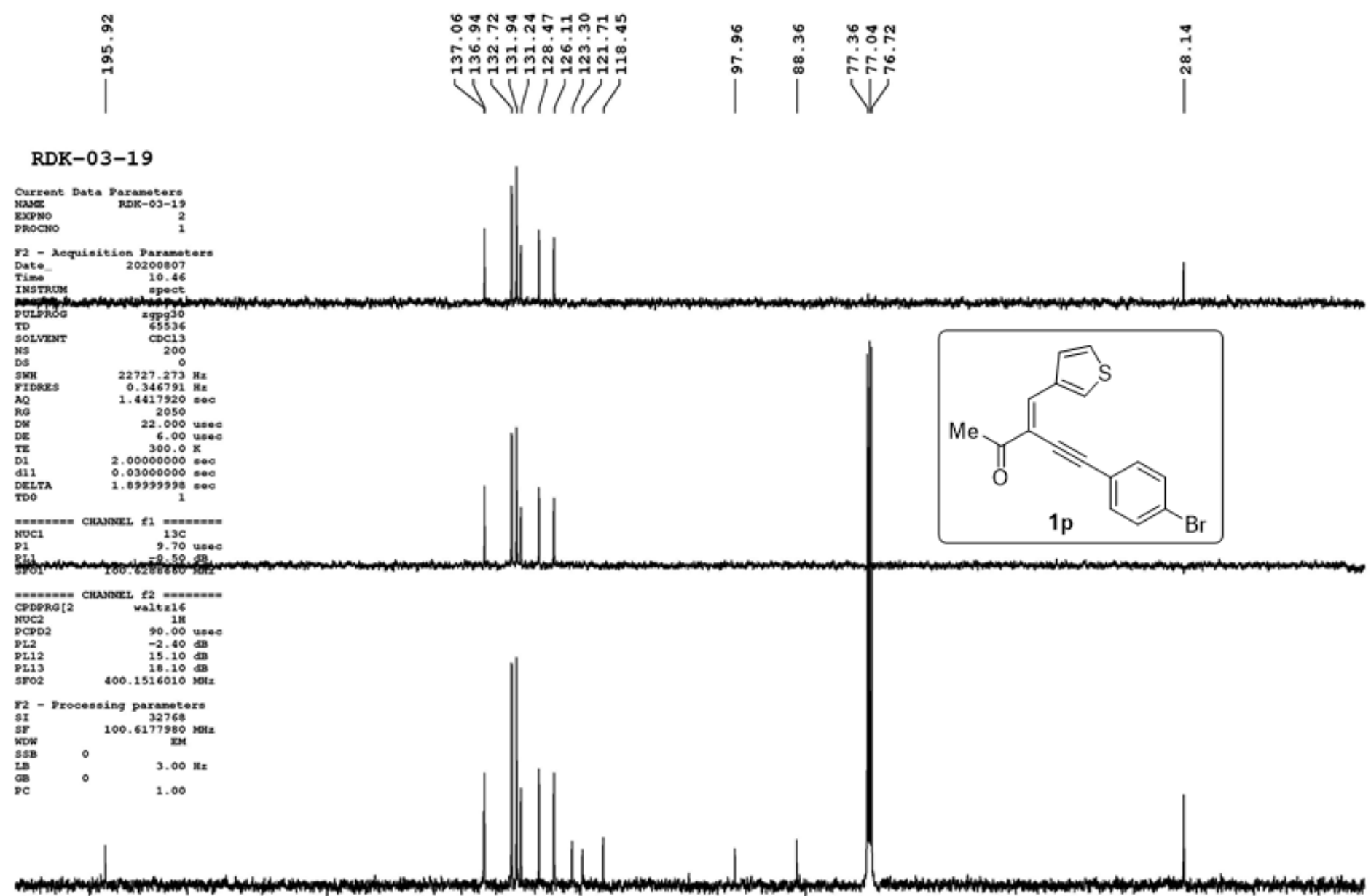

$\begin{array}{lllllllllllllllllllll}200 & 190 & 180 & 170 & 160 & 150 & 140 & 130 & 120 & 110 & 100 & 90 & 80 & 70 & 60 & 50 & 40 & 30 & 20 & \mathrm{ppm}\end{array}$ 
Solvent: $\mathrm{CDCl}_{3}$

SFO1: $400 \mathrm{MHz}$

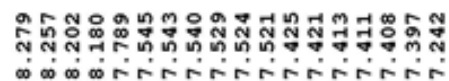

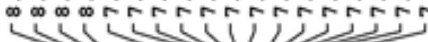

RDK-03-52

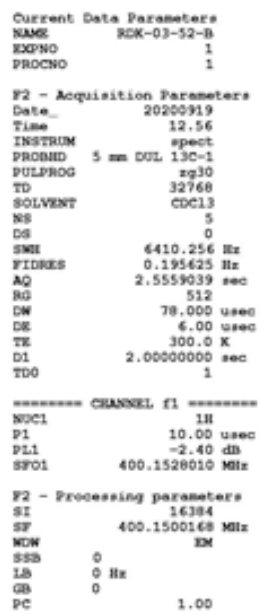

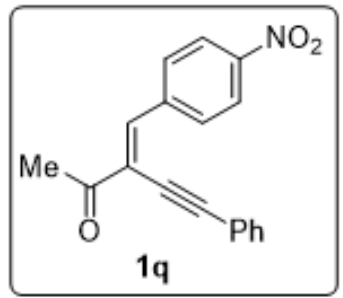


Solvent: $\mathrm{CDCl}_{3}$

SFO1: $125 \mathrm{MHz}$
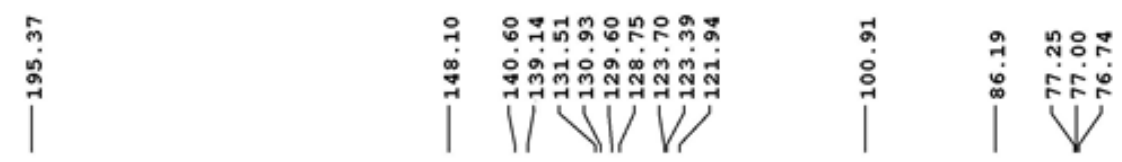

$\stackrel{m}{-}$

i

RDK-03-52-B

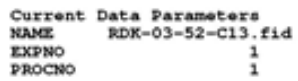

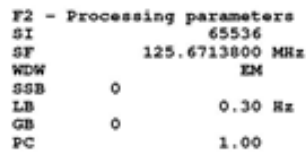
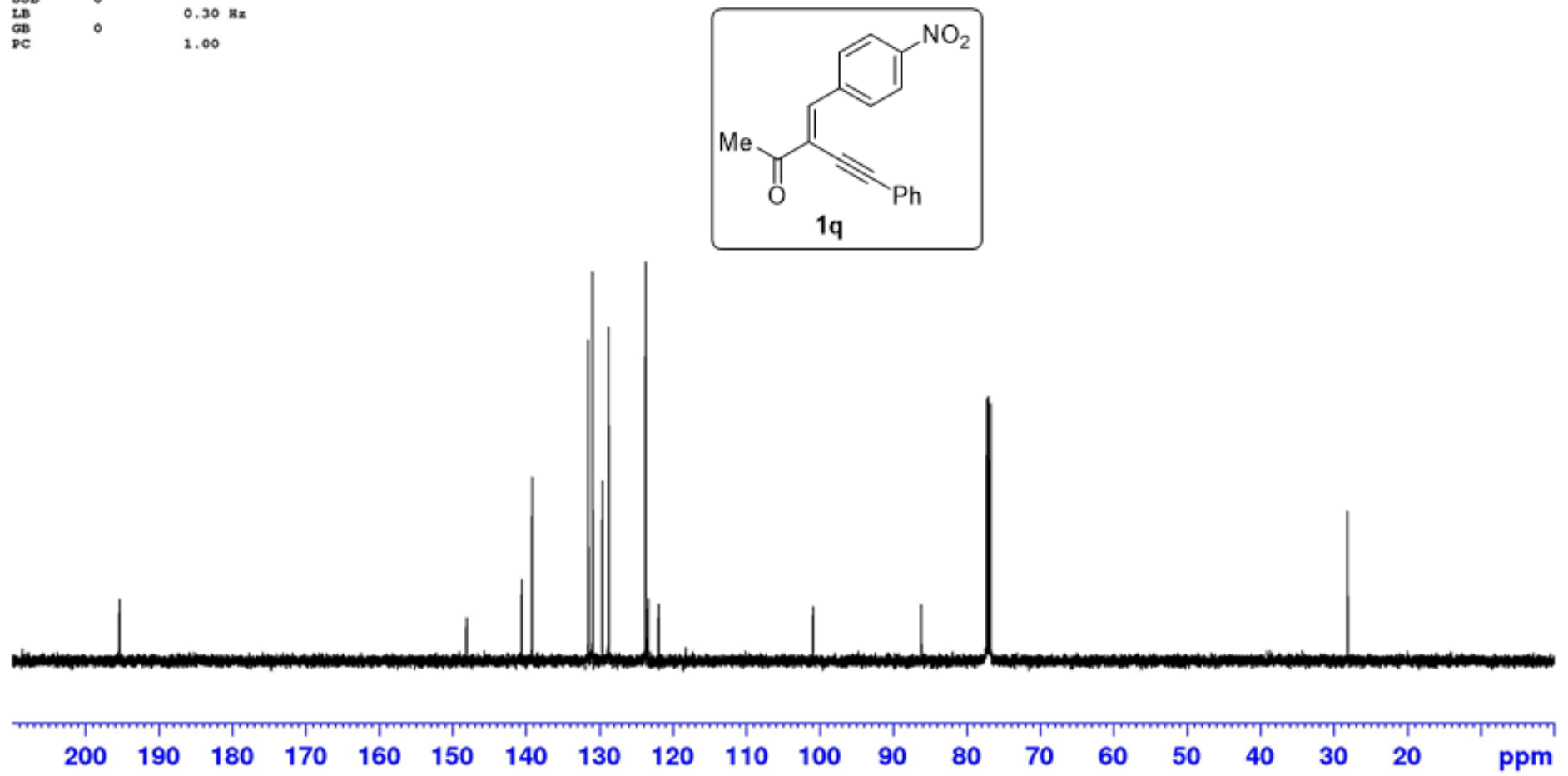
Solvent: $\mathrm{CDCl}_{3}$

SFO1: $600 \mathrm{MHz}$

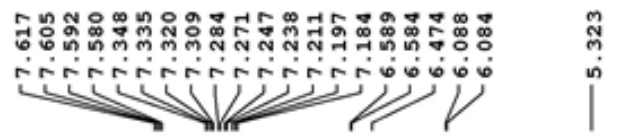

มู

RDK-02-182
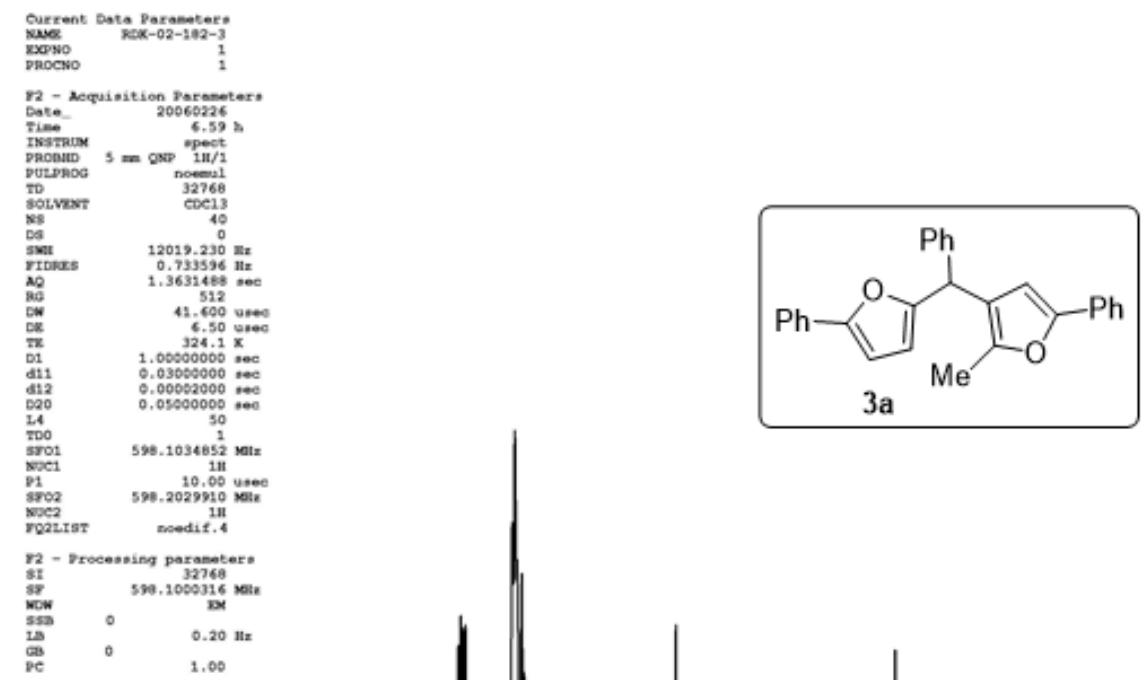

pe 1.00
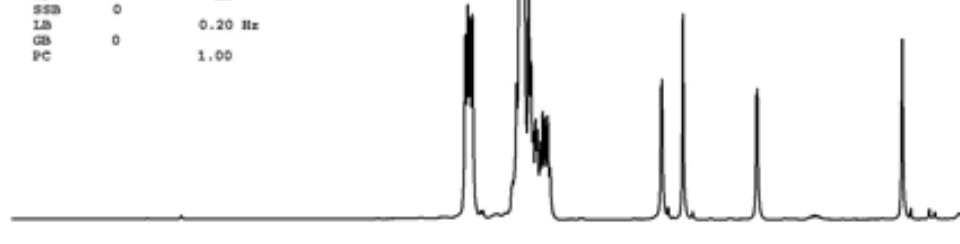

9.5

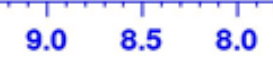

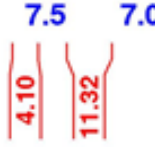

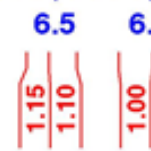

5.5 
Solvent: $\mathrm{CDCl}_{3}$

SFO1: $150 \mathrm{MHz}$
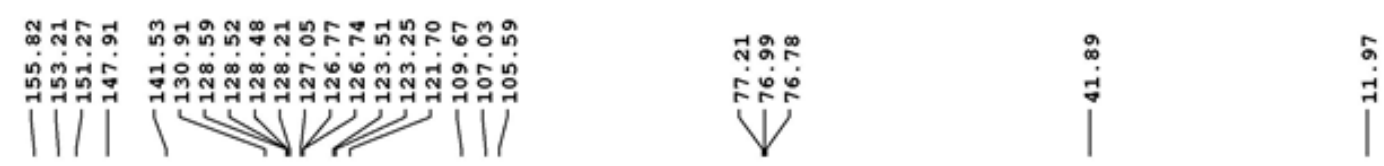

\section{RDK-02-182}

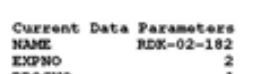

r2 - Aequideition parameter
Dato
202002020
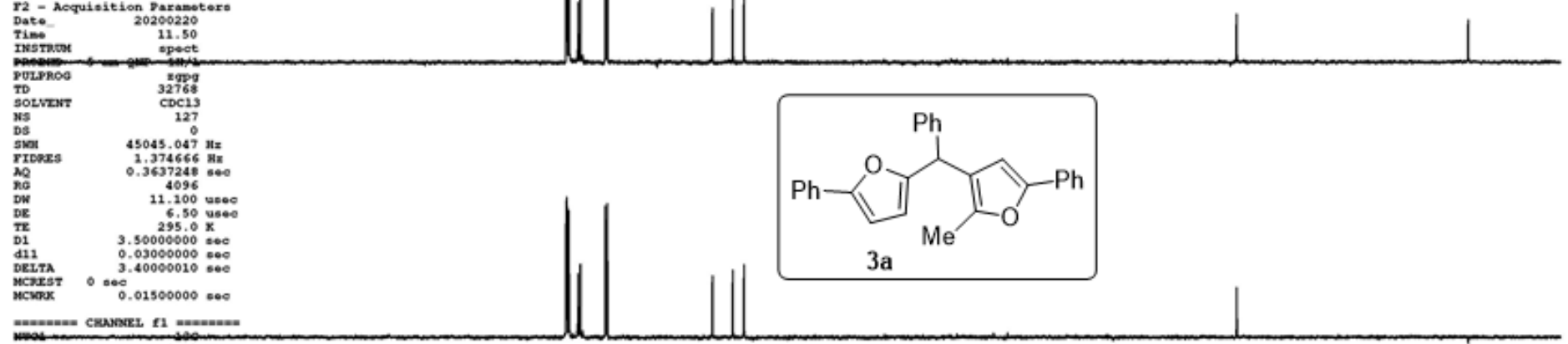

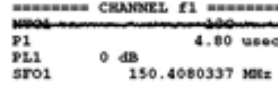

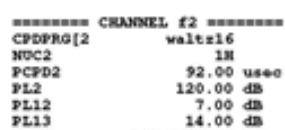

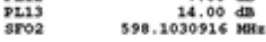

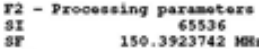

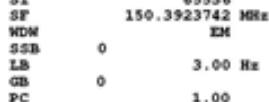

1.00 
Solvent: $\mathrm{CDCl}_{3}$

SFO1: $400 \mathrm{MHz}$

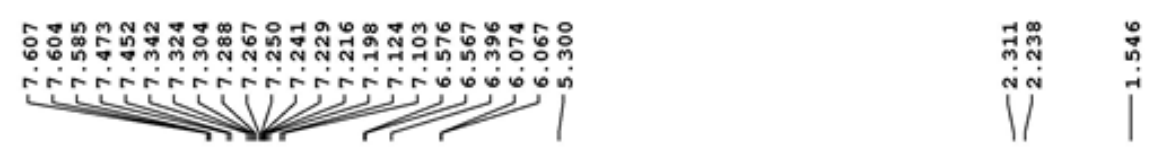

RDK-02-183-F
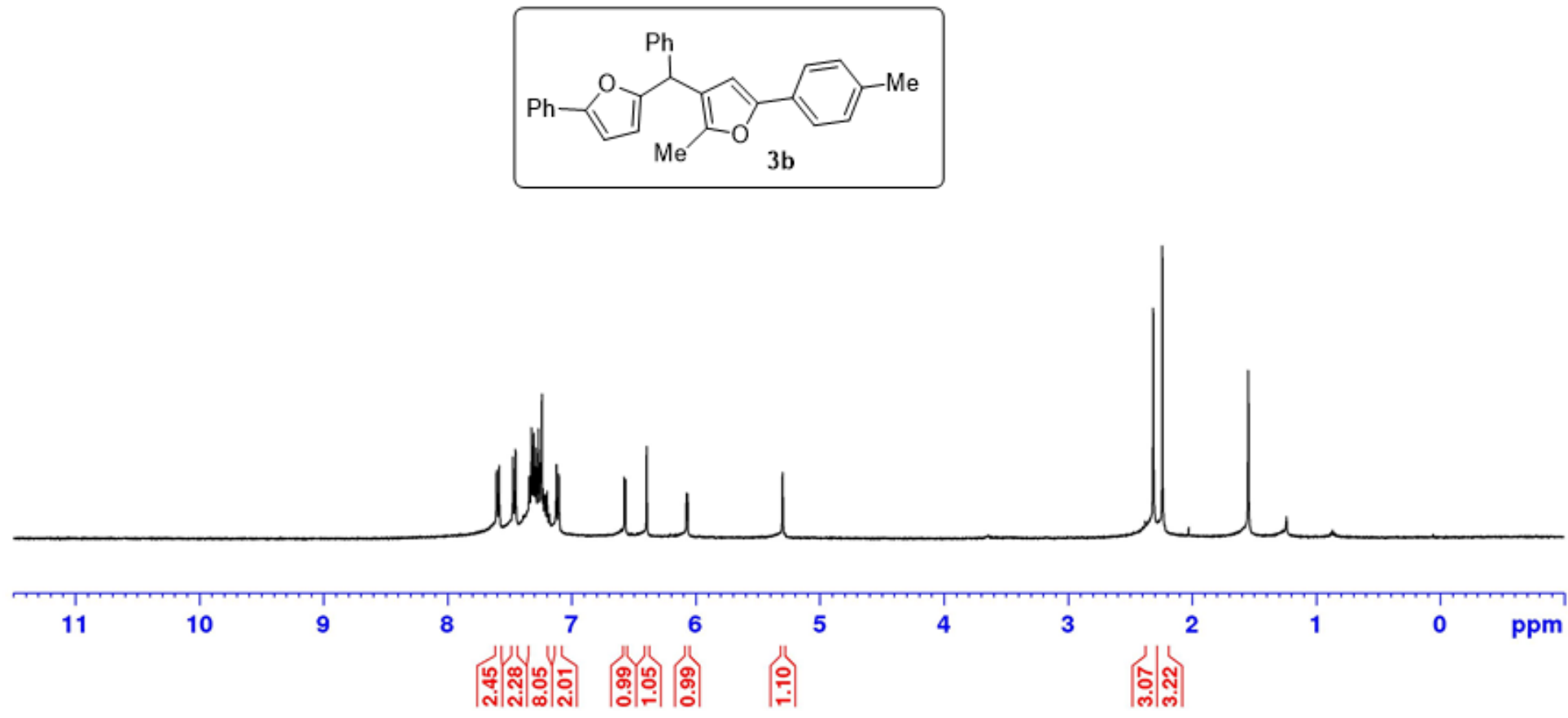
Solvent: $\mathrm{CDCl}_{3}$

SFO1: $125 \mathrm{MHz}$

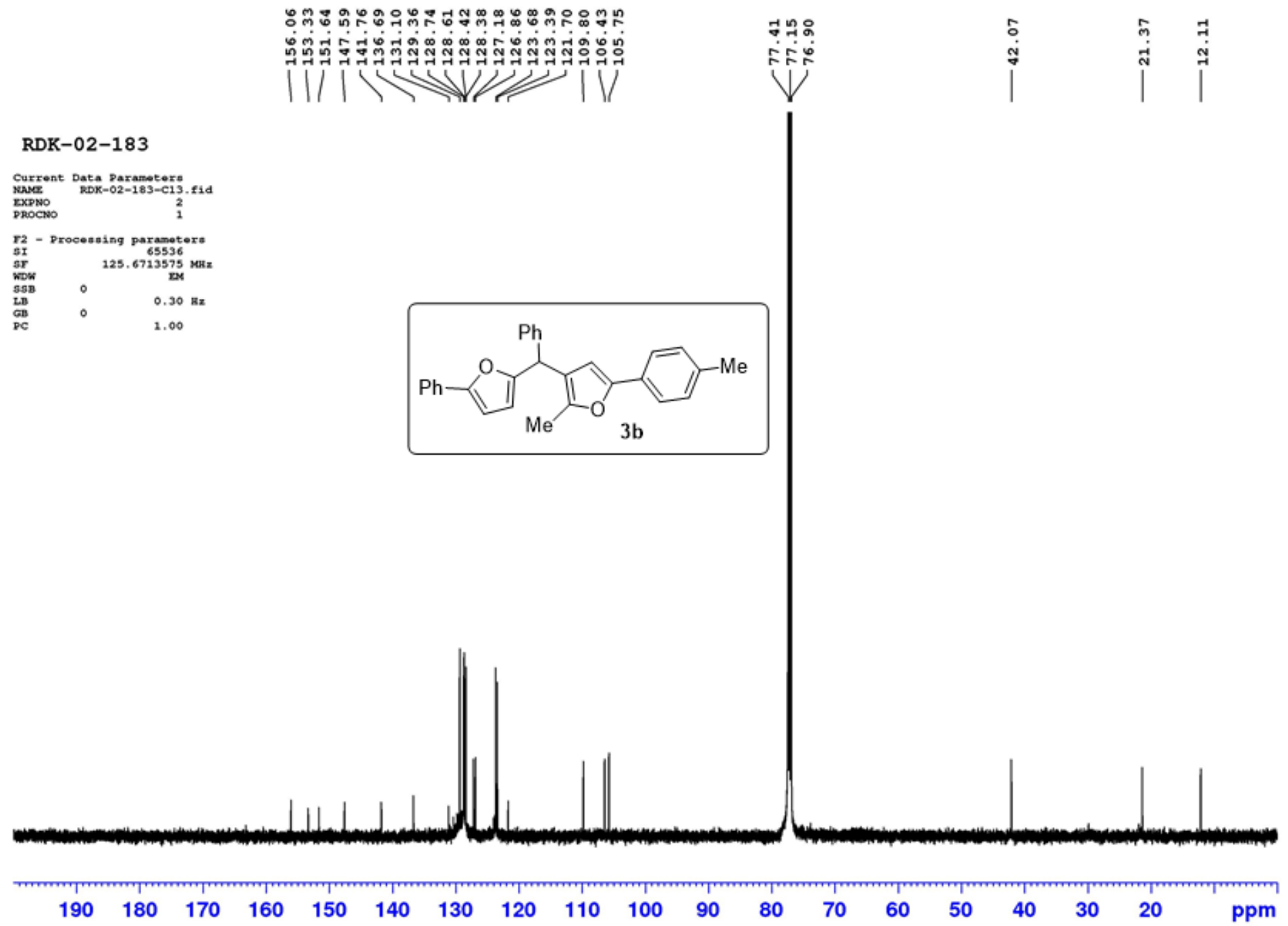


Solvent: $\mathrm{CDCl}_{3}$

SFO1: $700 \mathrm{MHz}$
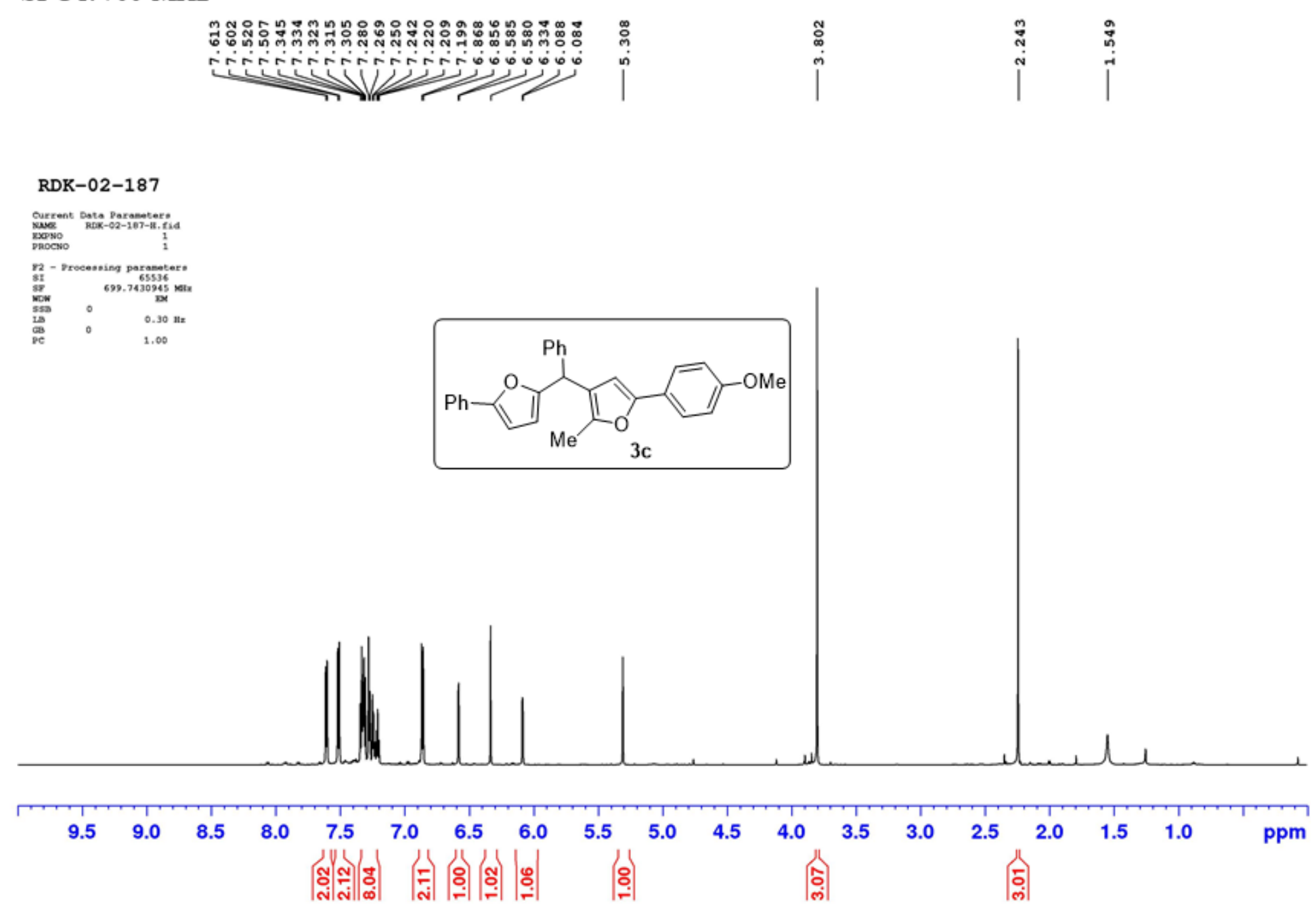

S63 
Solvent: $\mathrm{CDCl}_{3}$

SFO1: $175 \mathrm{MHz}$

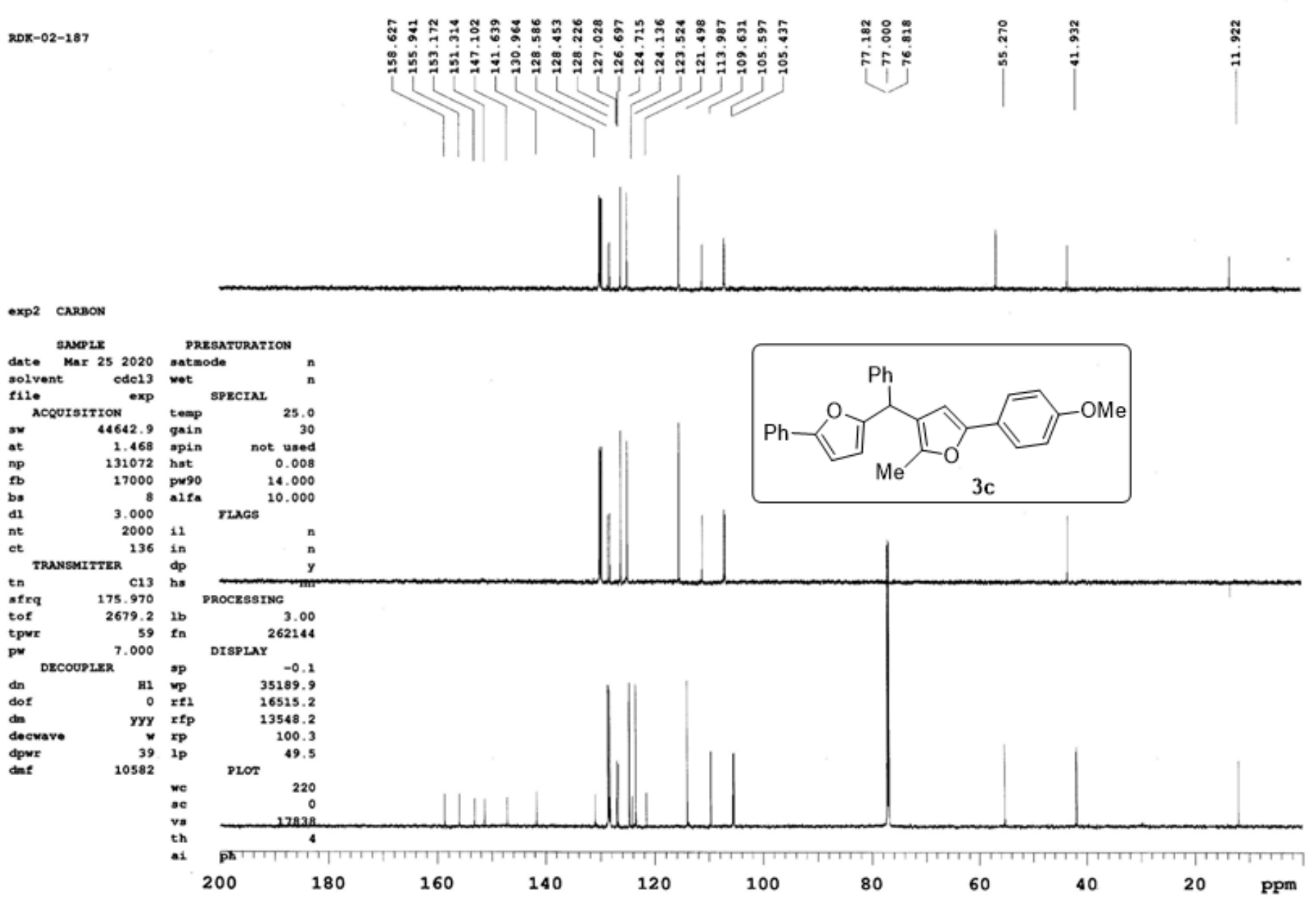


Solvent: $\mathrm{CDCl}_{3}$

SFO1: $400 \mathrm{MHz}$

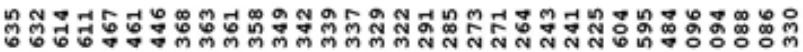

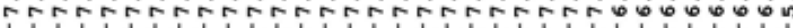

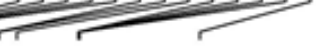

RDK-02-186

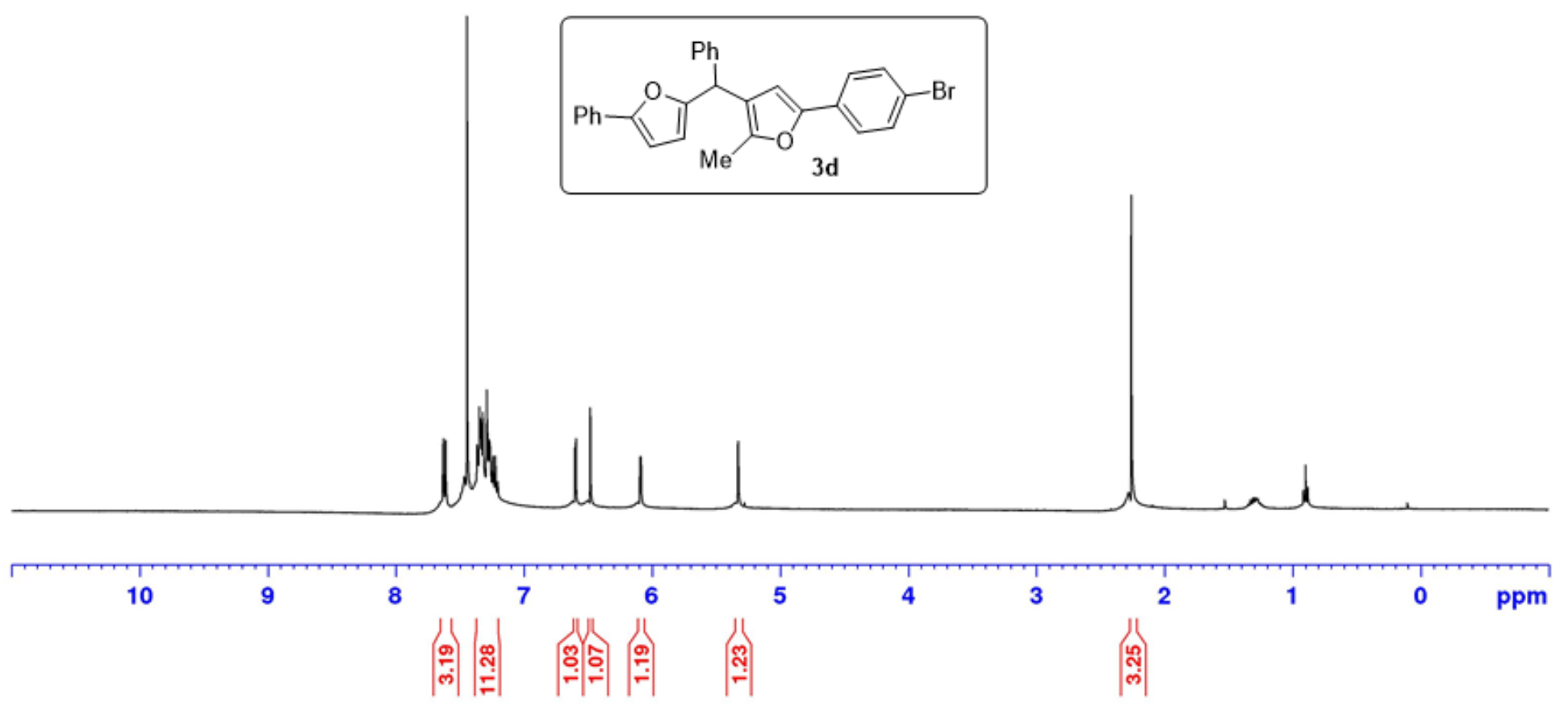


Solvent: $\mathrm{CDCl}_{3}$

SFO1: $100 \mathrm{MHz}$

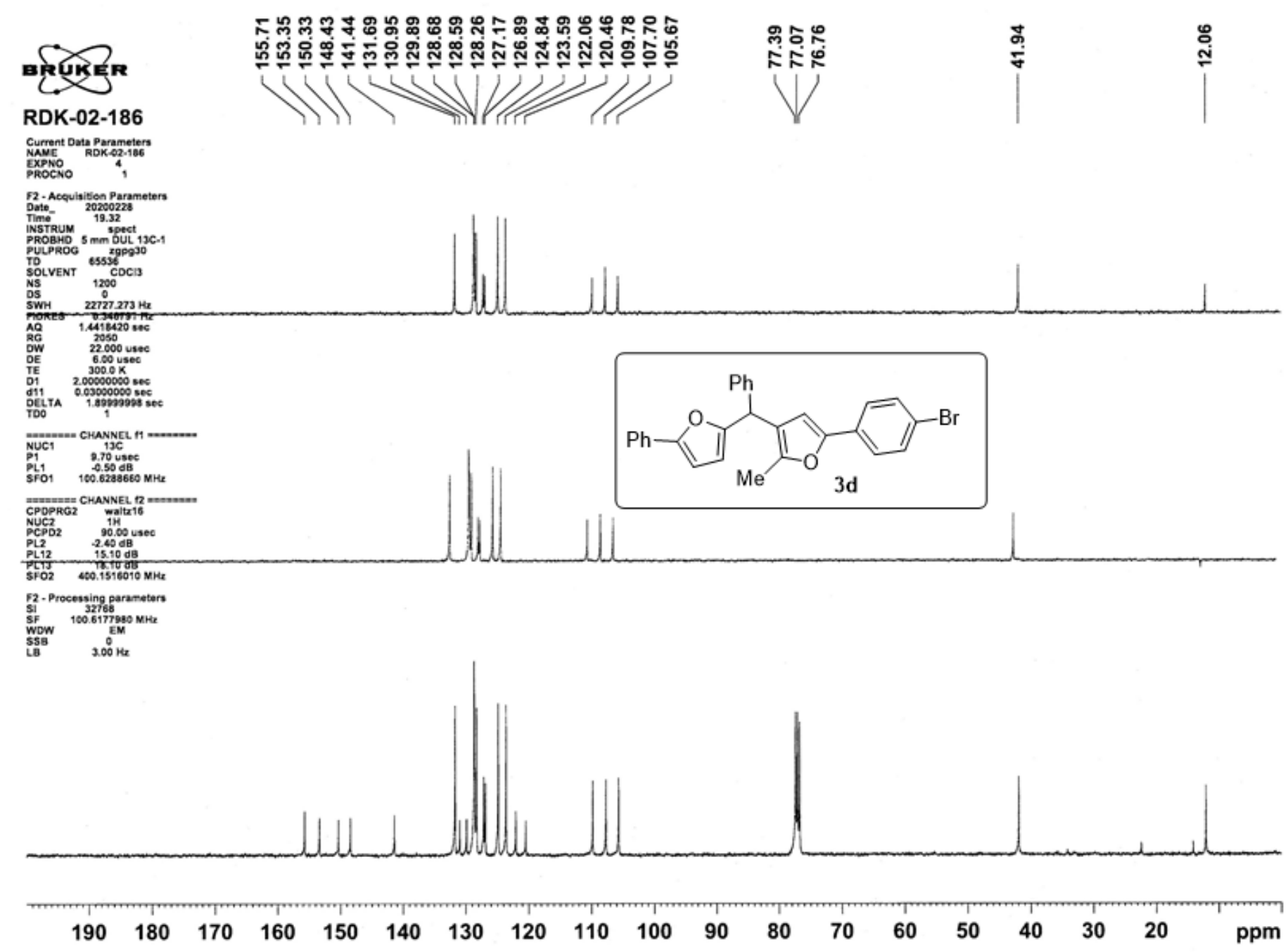


Solvent: $\mathrm{CDCl}_{3}$

SFO1: $600 \mathrm{MHz}$

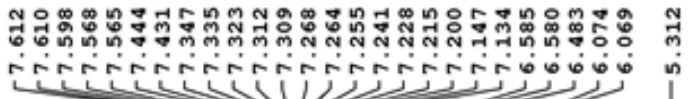

RDK-02-185
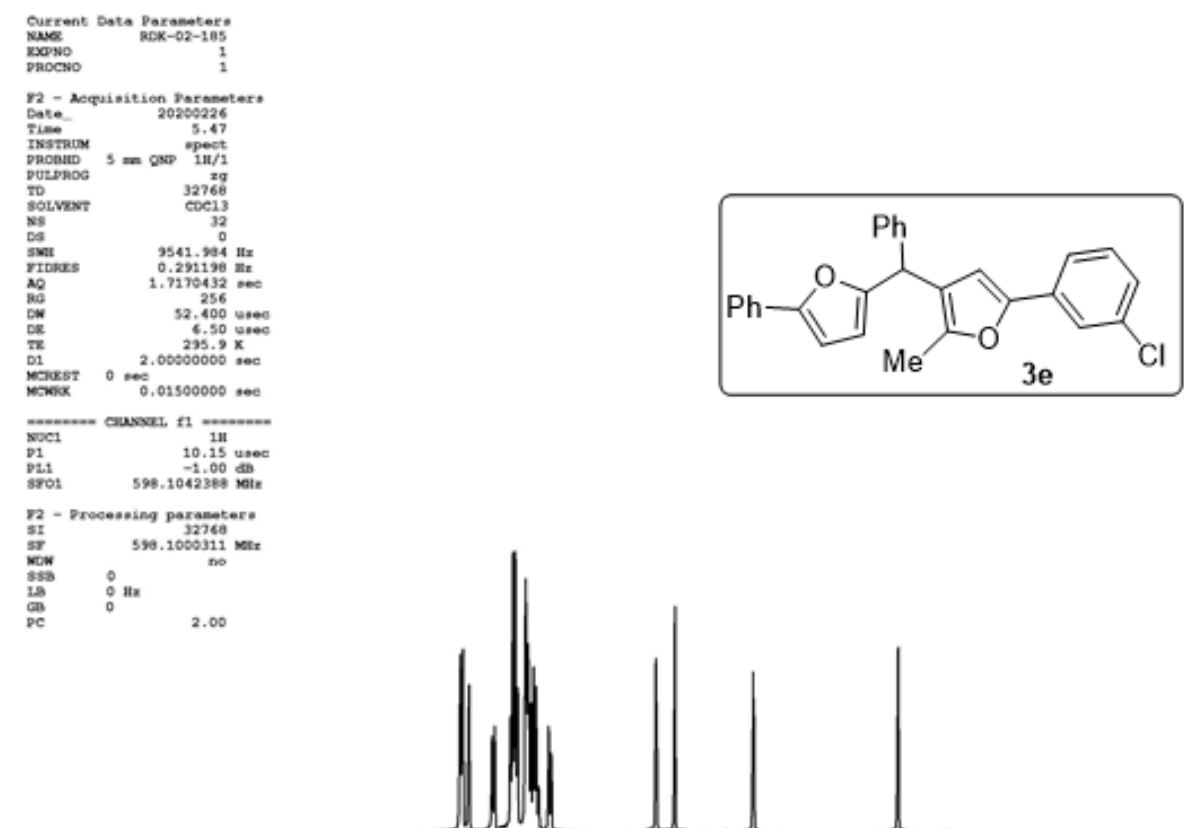

9.5

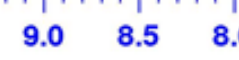

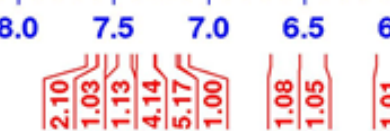

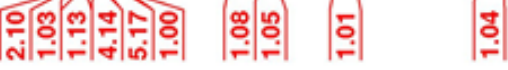


Solvent: $\mathrm{CDCl}_{3}$ SFO1: $150 \mathrm{MHz}$
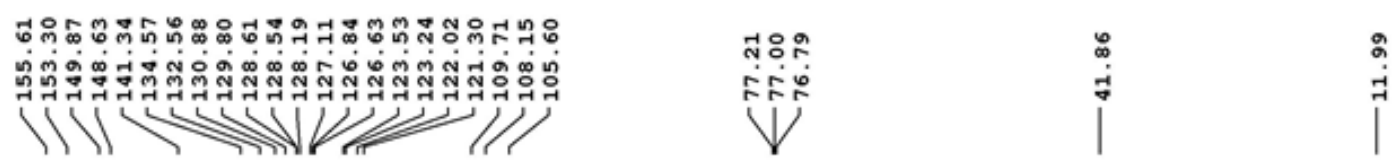

\section{RDK-02-185}

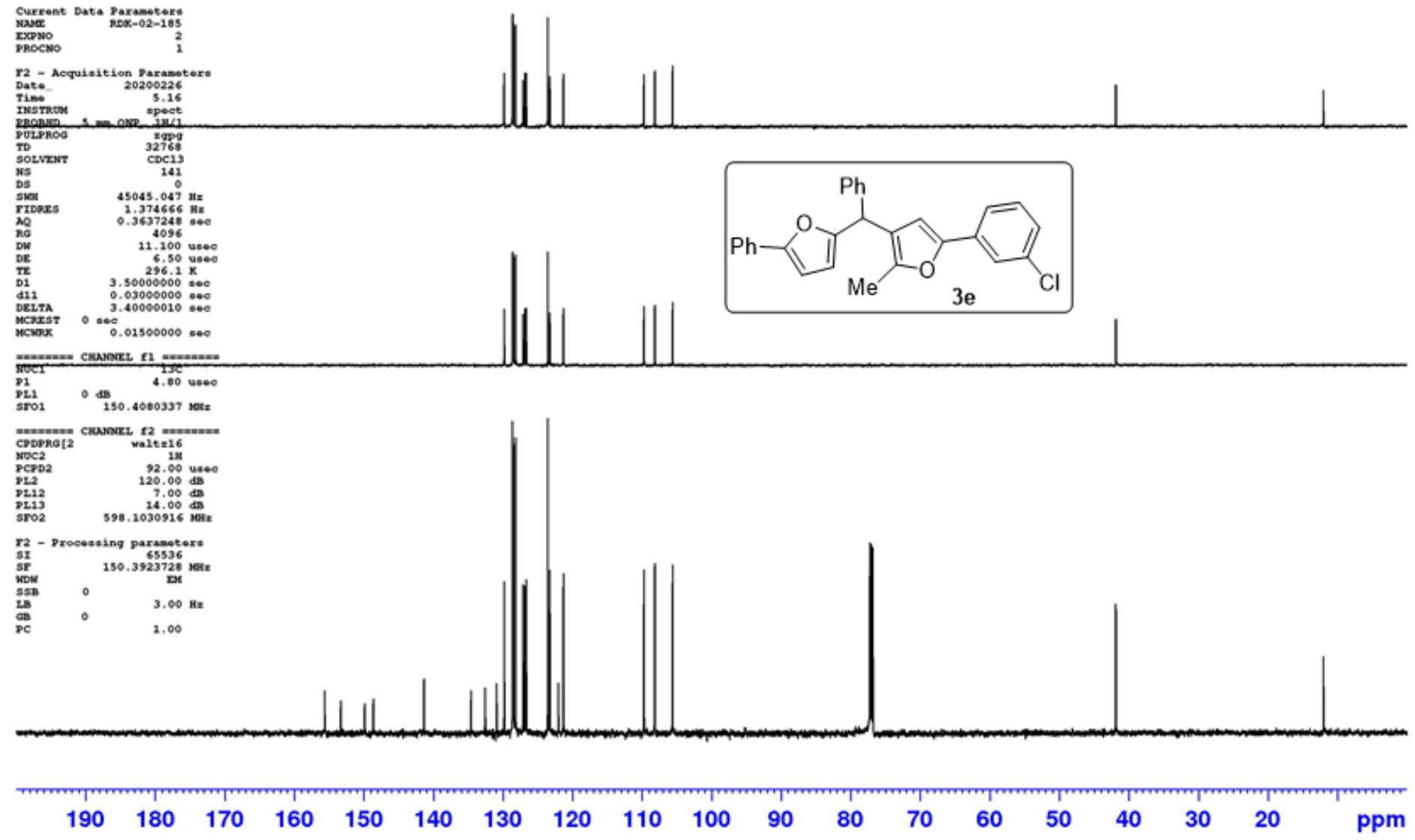


Solvent: $\mathrm{CDCl}_{3}$

SFO1: $400 \mathrm{MHz}$

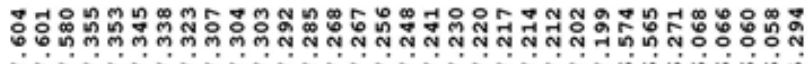

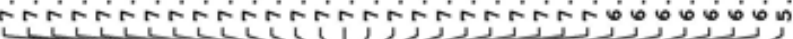

RDK-02-201-P

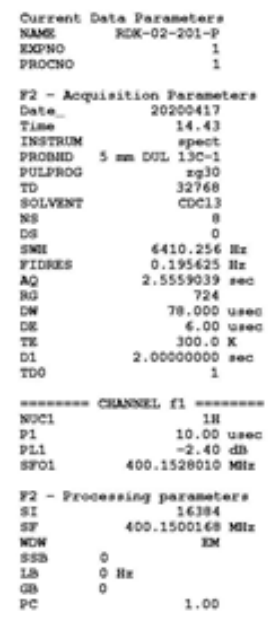
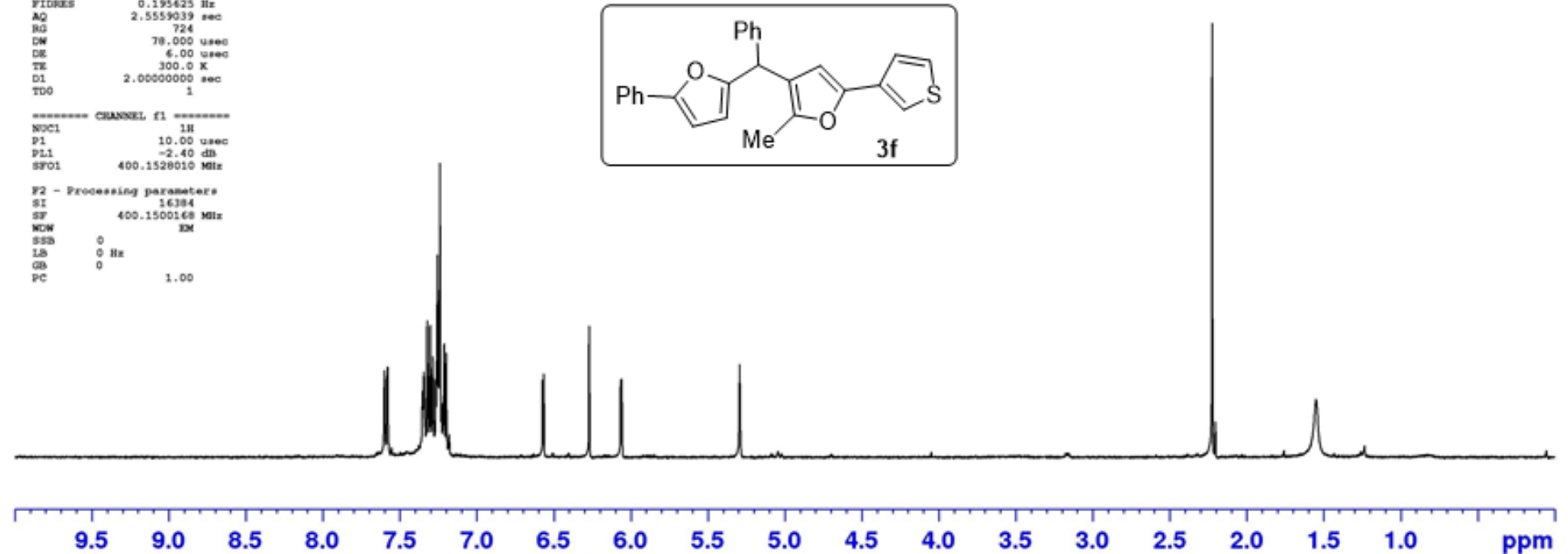

9.5

9.08 .5

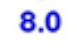

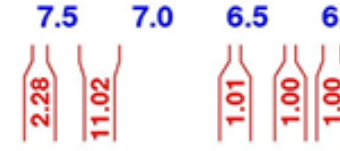

5.5

:

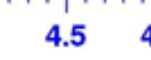


Solvent: $\mathrm{CDCl}_{3}$ SFO1: $150 \mathrm{MHz}$

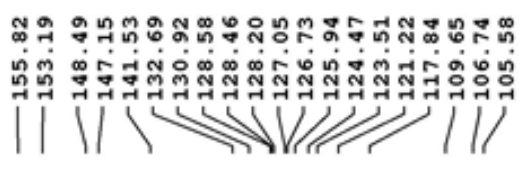

RDK-02-201

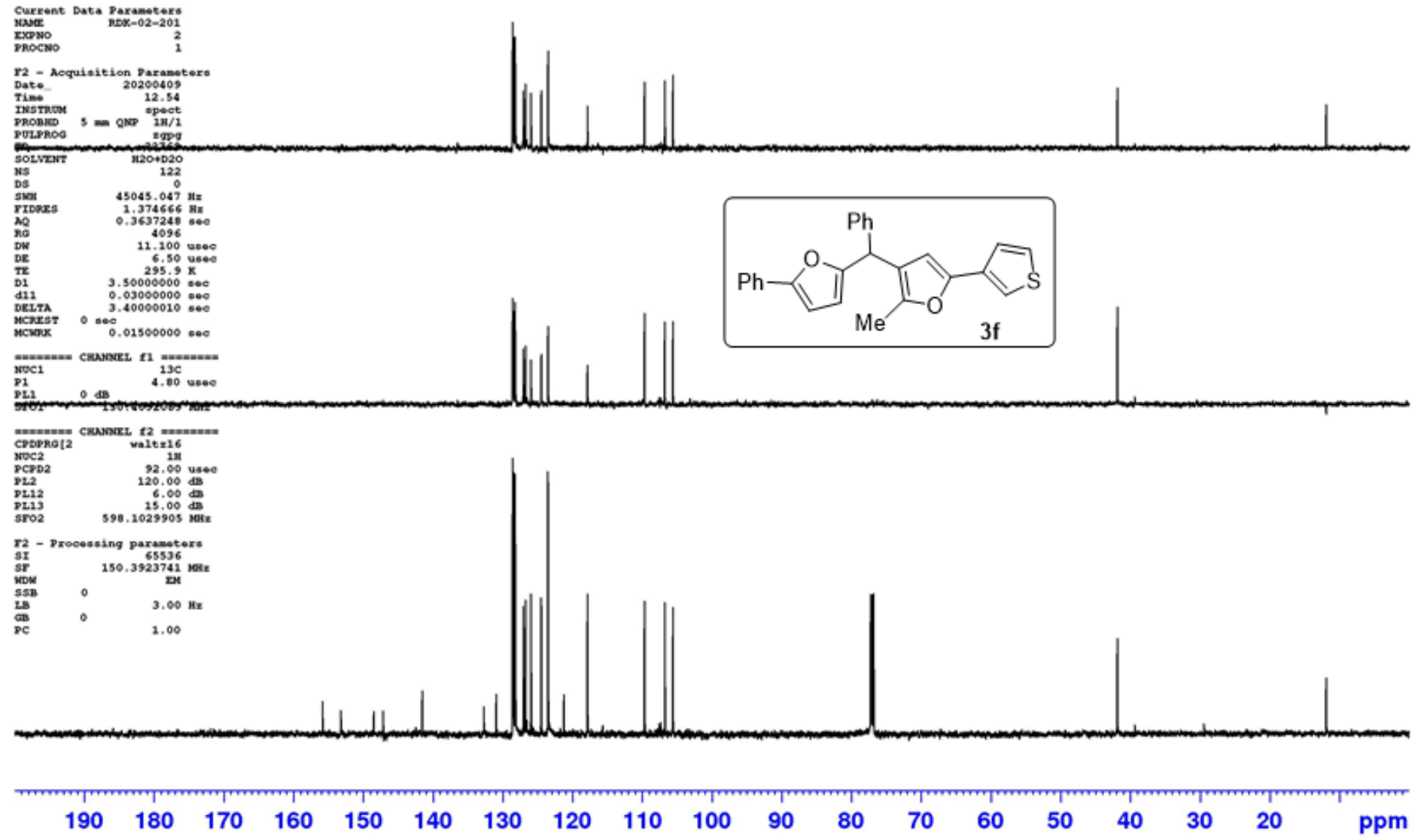


Solvent: $\mathrm{CDCl}_{3}$ SFO1: $700 \mathrm{MHz}$

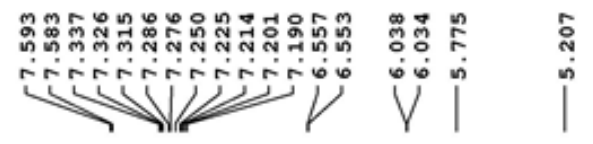

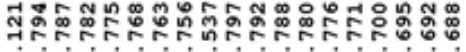

(1)

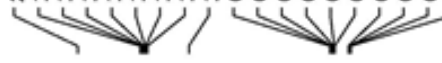

RDK-02-196

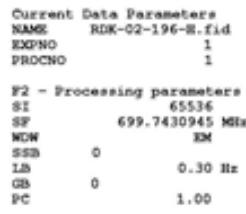
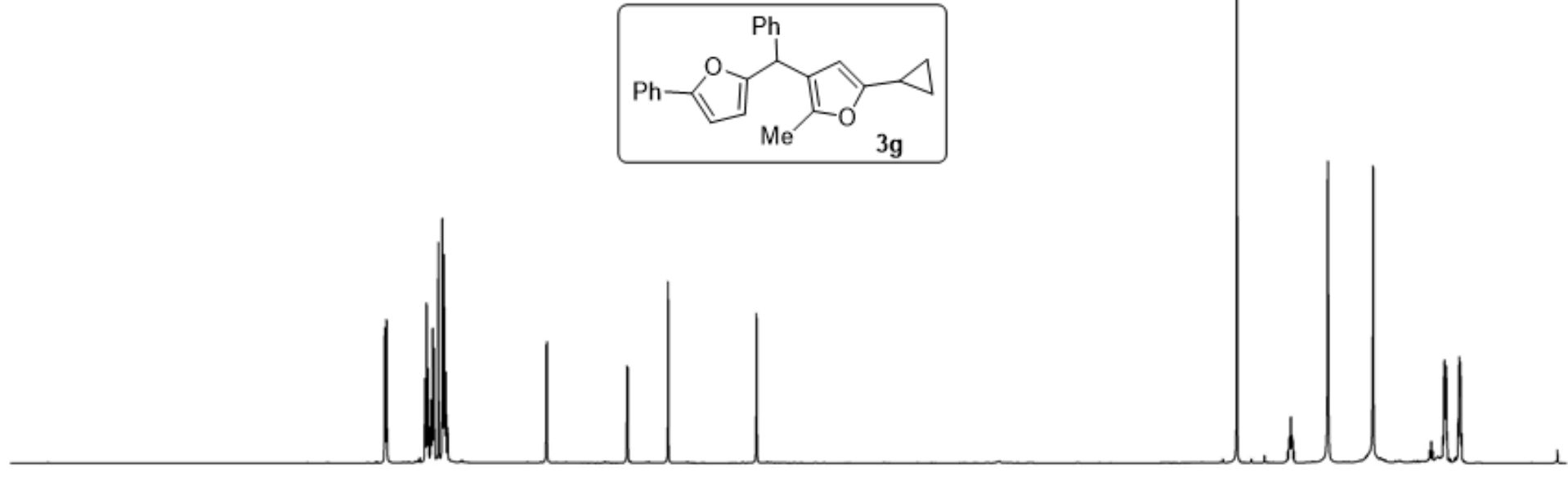

$\begin{array}{llll}9.5 & 9.0 & 8.5 & 8\end{array}$

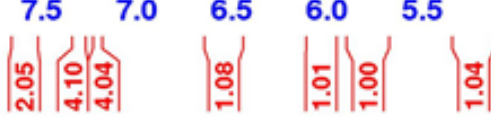

4.5
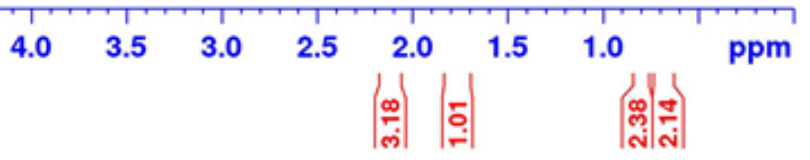
Solvent: $\mathrm{CDCl}_{3}$

SFO1: $175 \mathrm{MHz}$

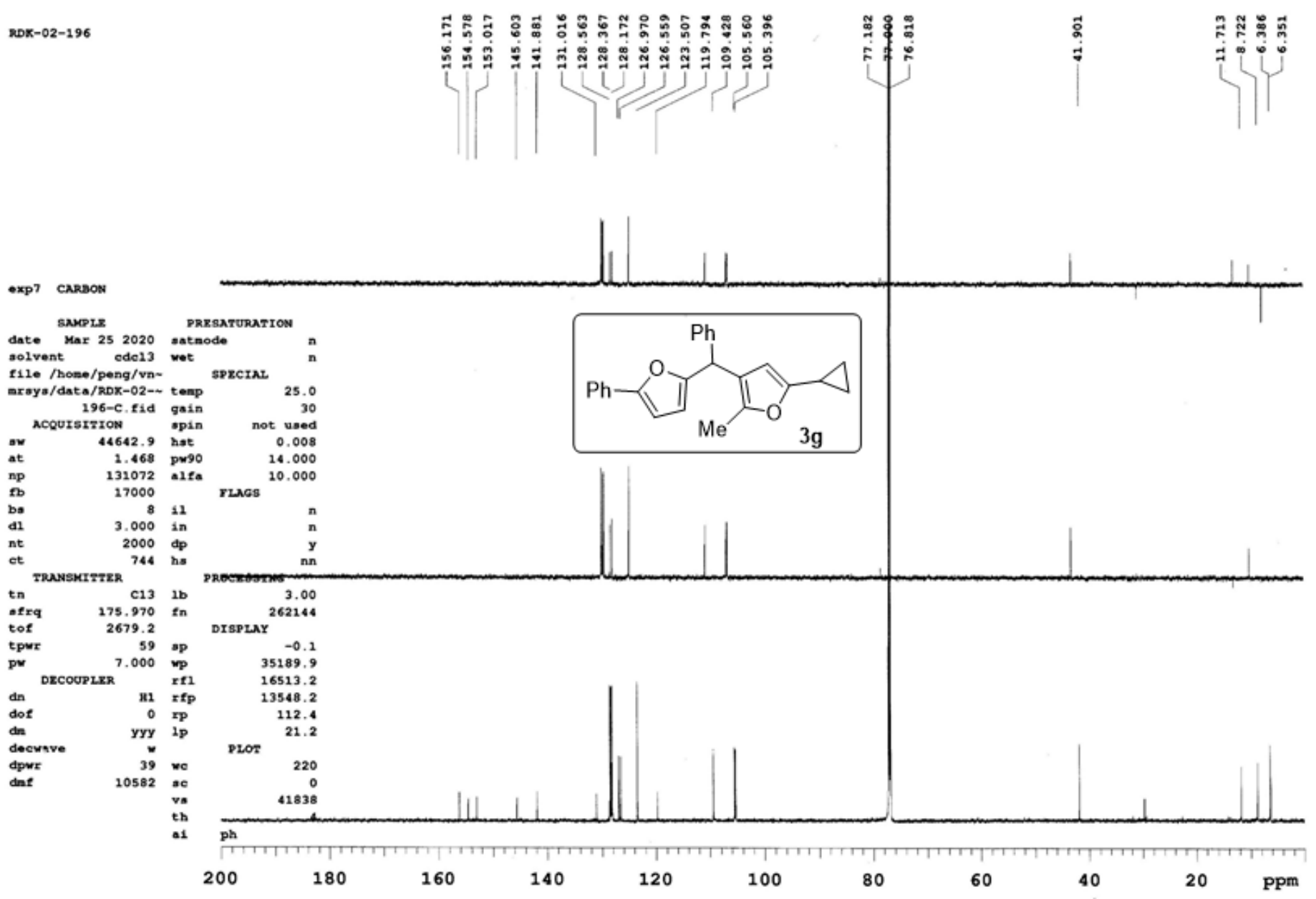


Solvent: $\mathrm{CDCl}_{3}$

SFO1: $600 \mathrm{MHz}$

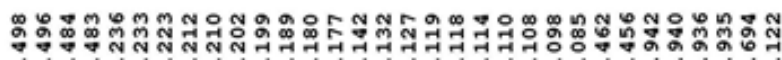

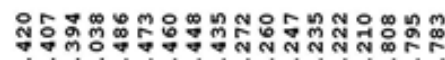

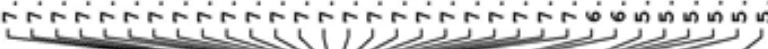

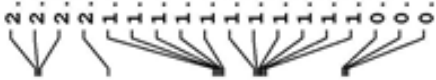

RDK-02-197

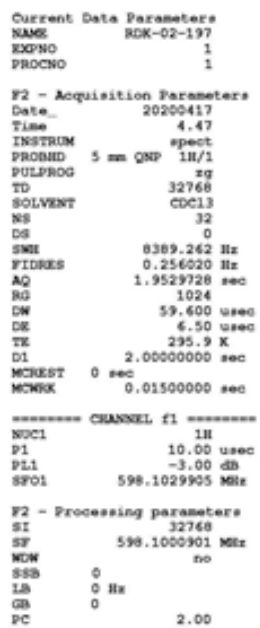
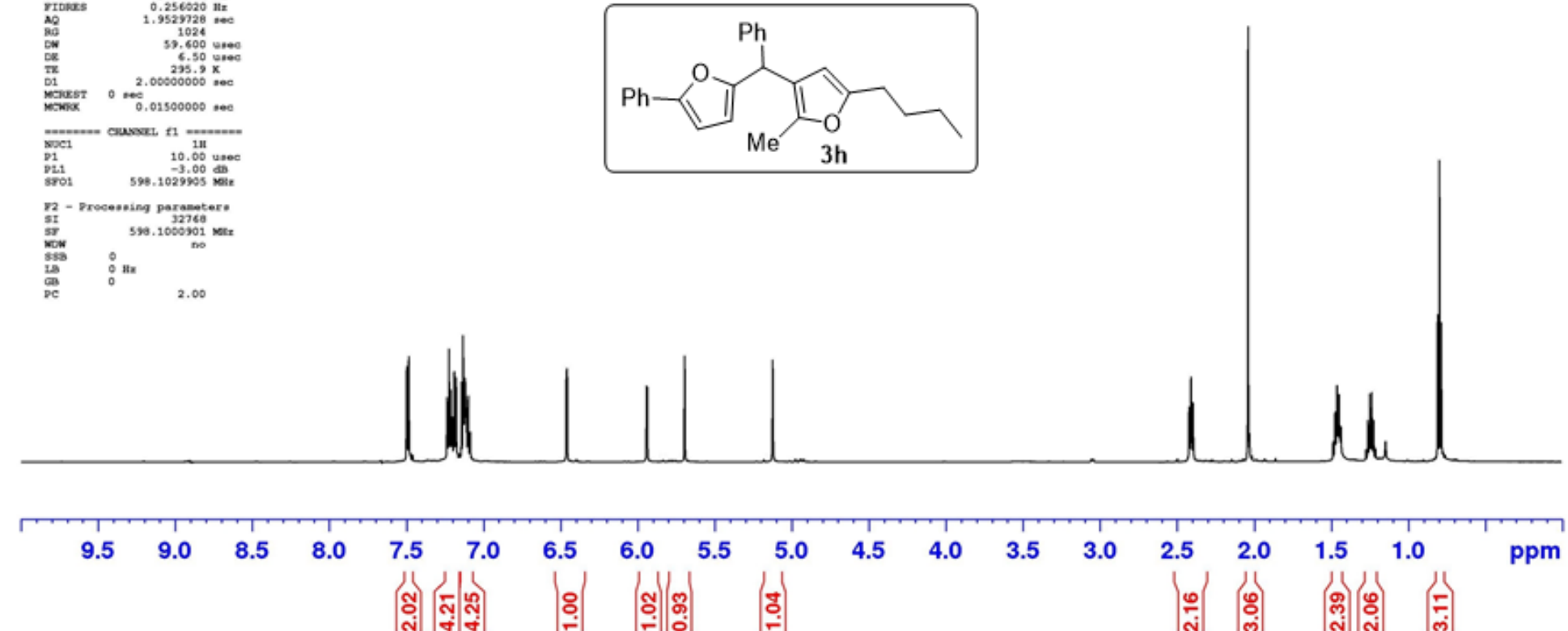
Solvent: $\mathrm{CDCl}_{3}$

SFO1: $150 \mathrm{MHz}$

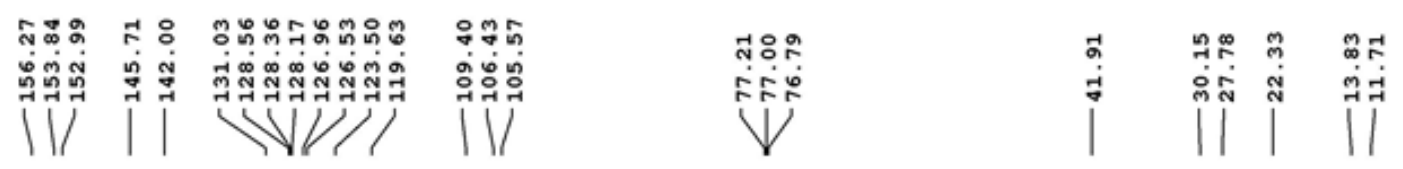

RDK-02-197

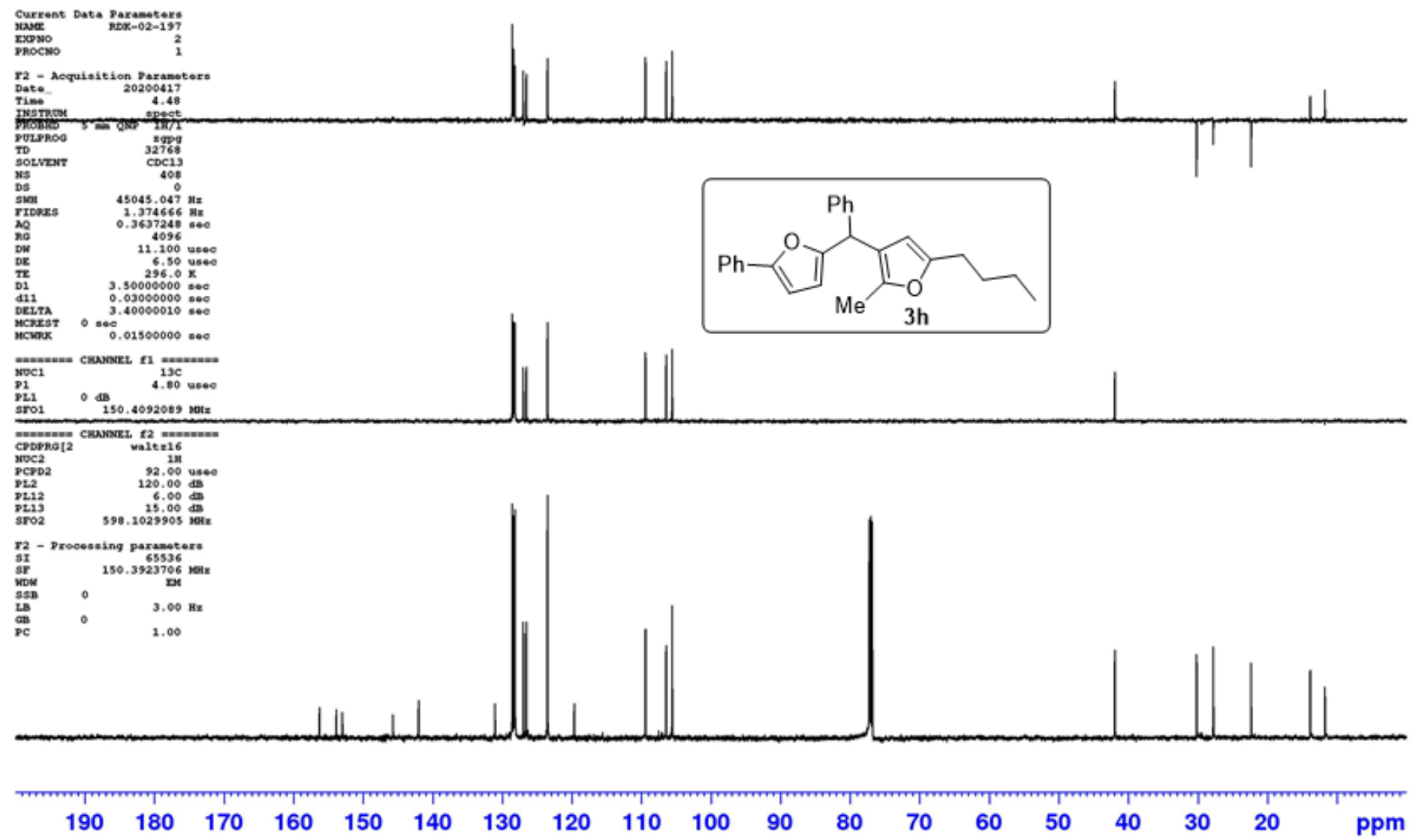


Solvent: $\mathrm{CDCl}_{3}$

SFO1: $400 \mathrm{MHz}$

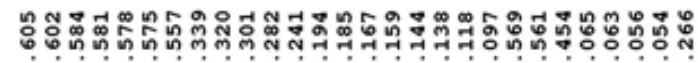

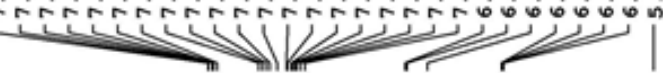

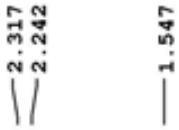

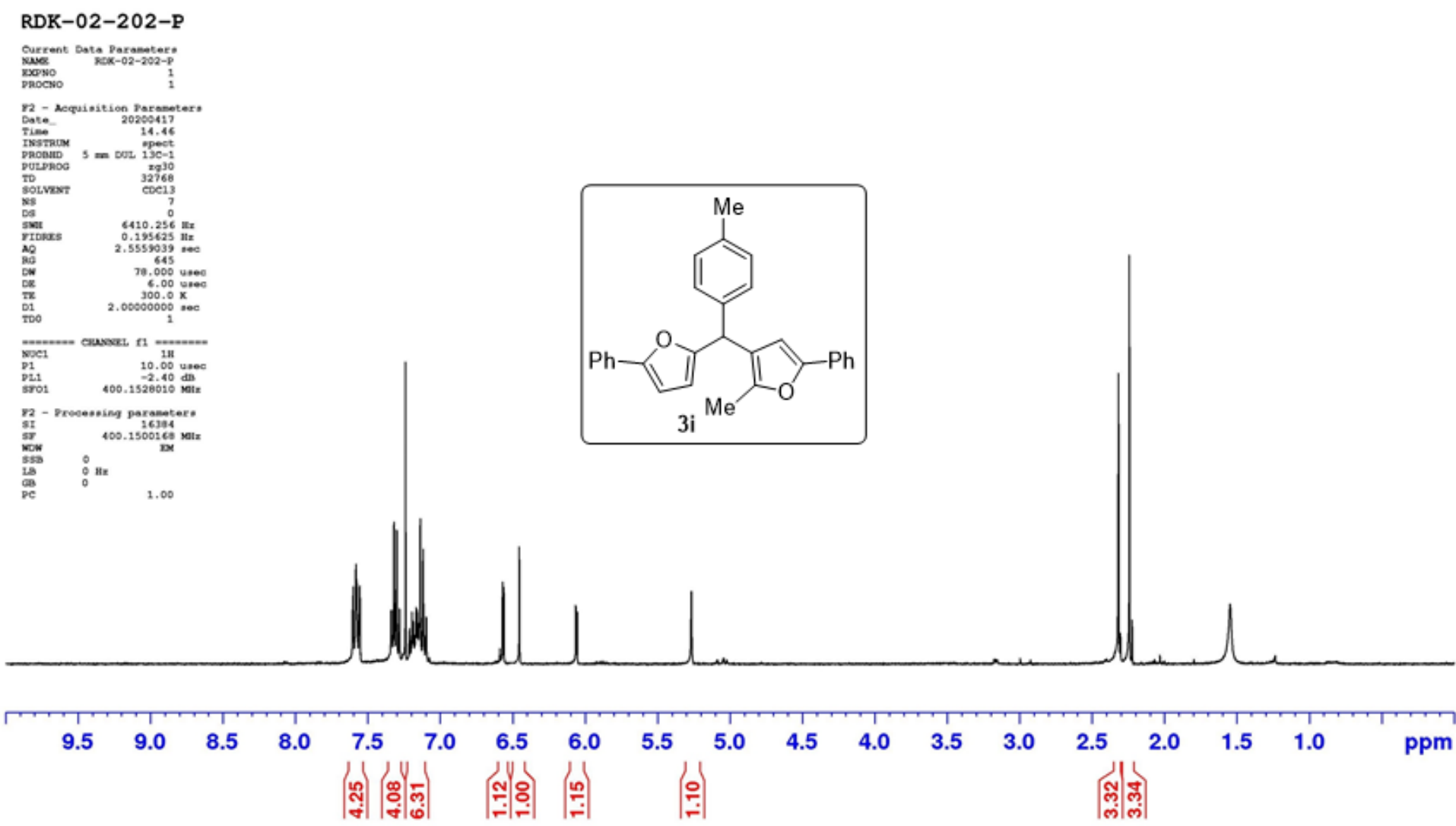


Solvent: $\mathrm{CDCl}_{3}$ SFO1: $150 \mathrm{MHz}$
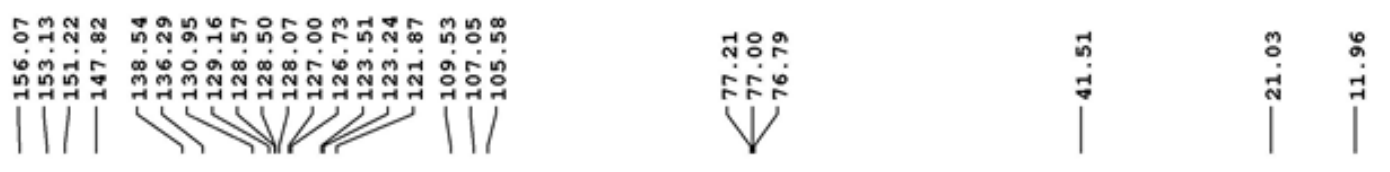

\section{RDK-02-202}

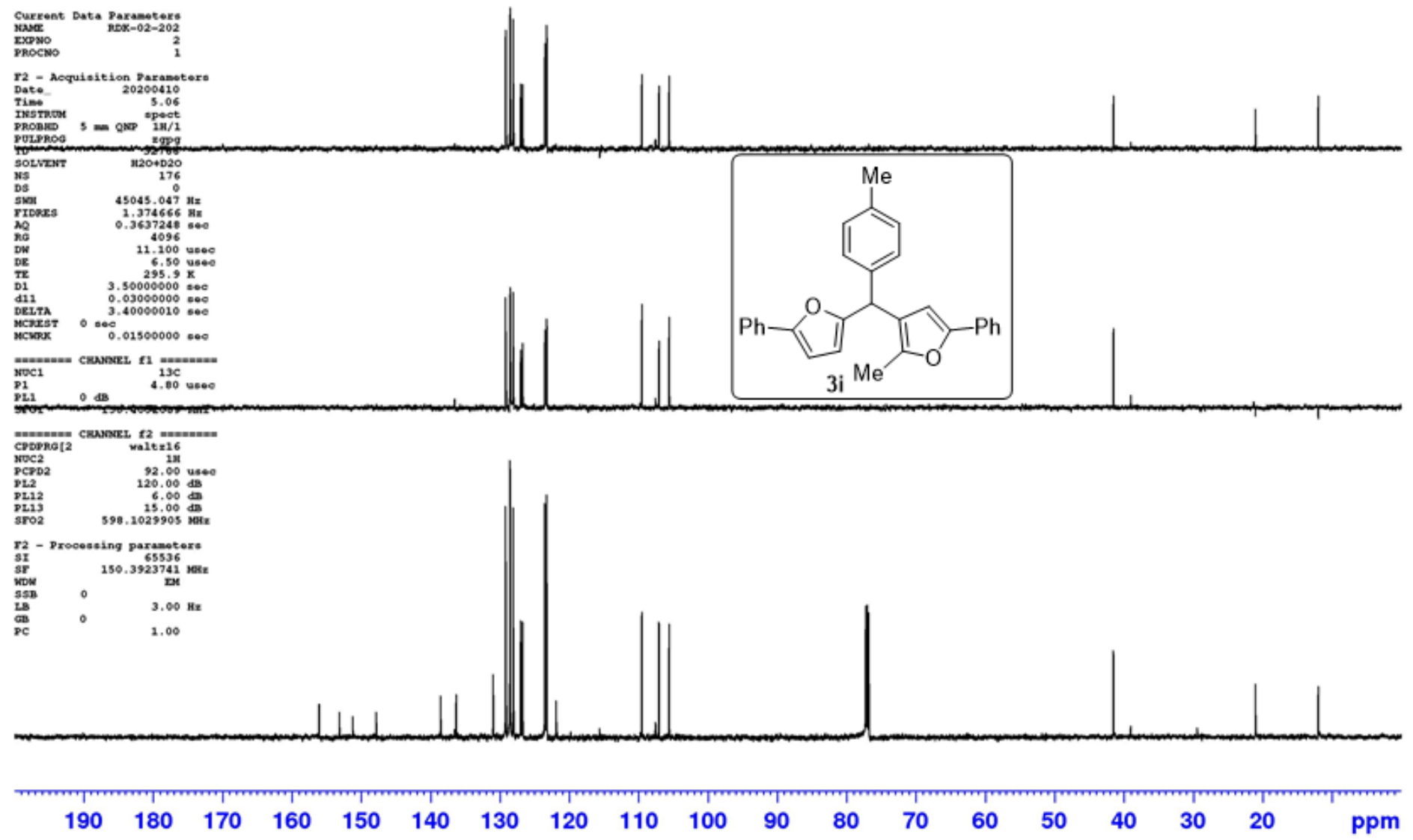


Solvent: $\mathrm{CDCl}_{3}$

SFO1: $600 \mathrm{MHz}$

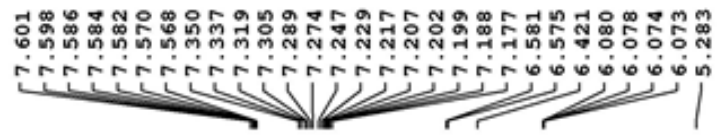

RDK-02-208
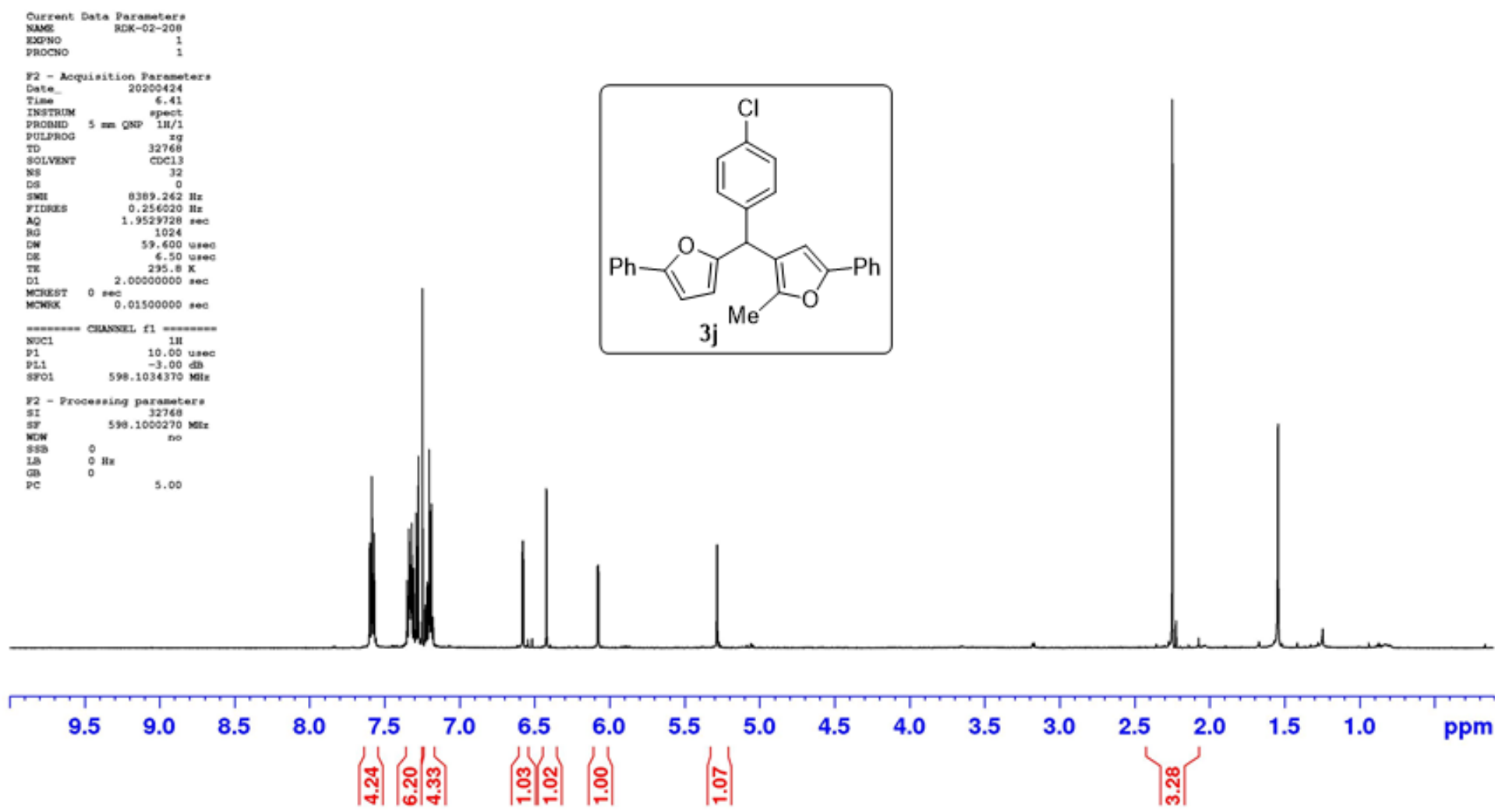
Solvent: $\mathrm{CDCl}_{3}$

SFO1: $150 \mathrm{MHz}$
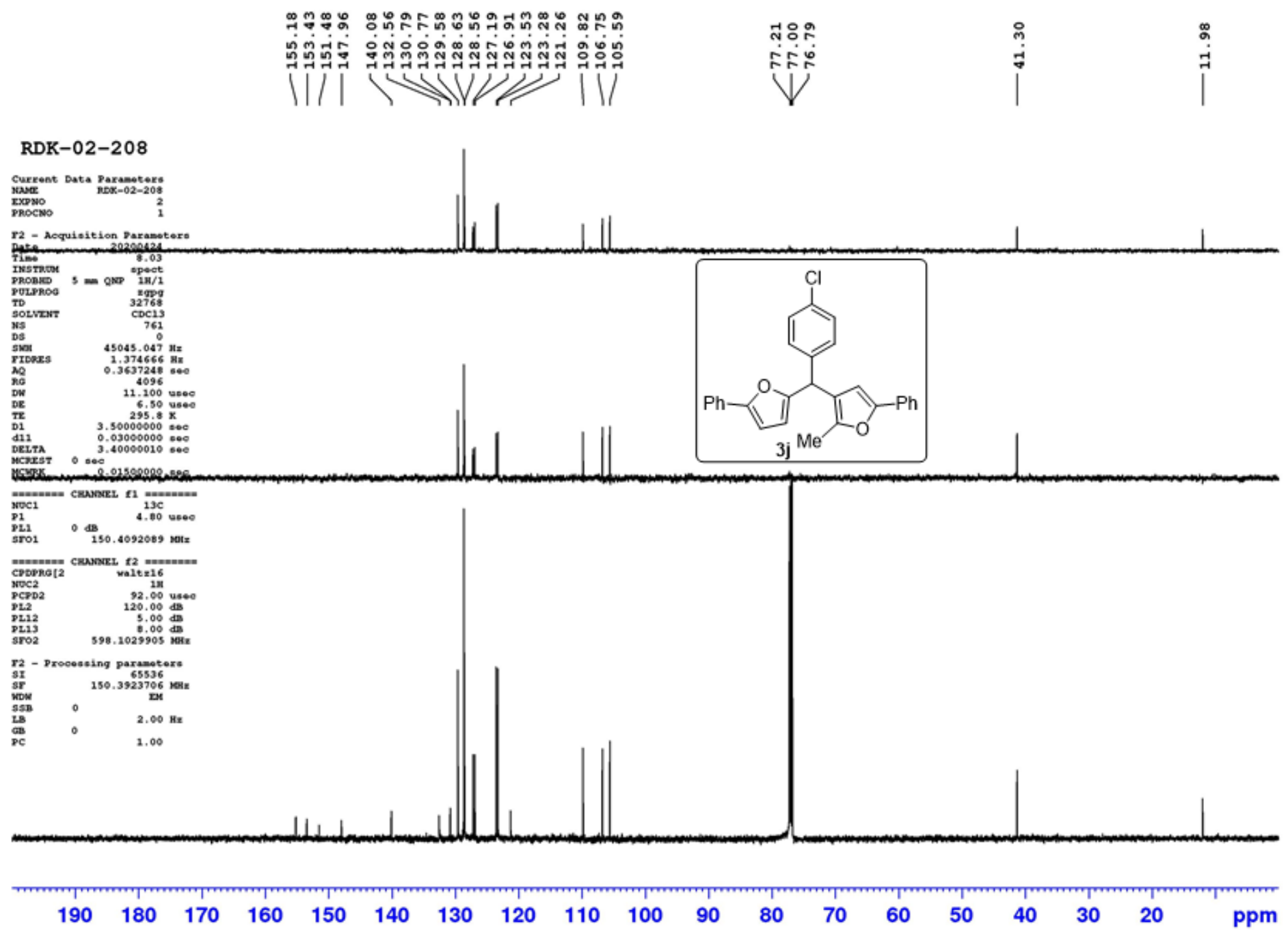
Solvent: $\mathrm{CDCl}_{3}$

SFO1: $400 \mathrm{MHz}$

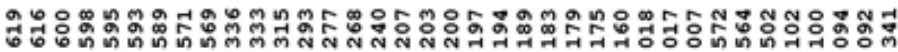

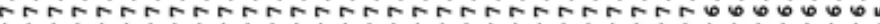

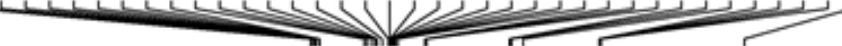

RDK-02-206-P

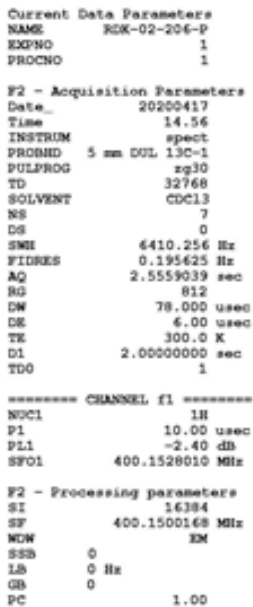

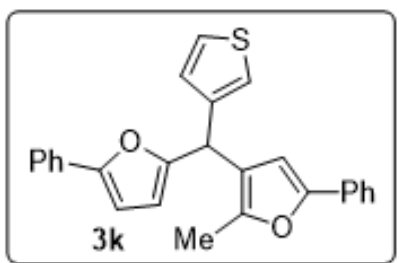

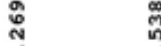

i

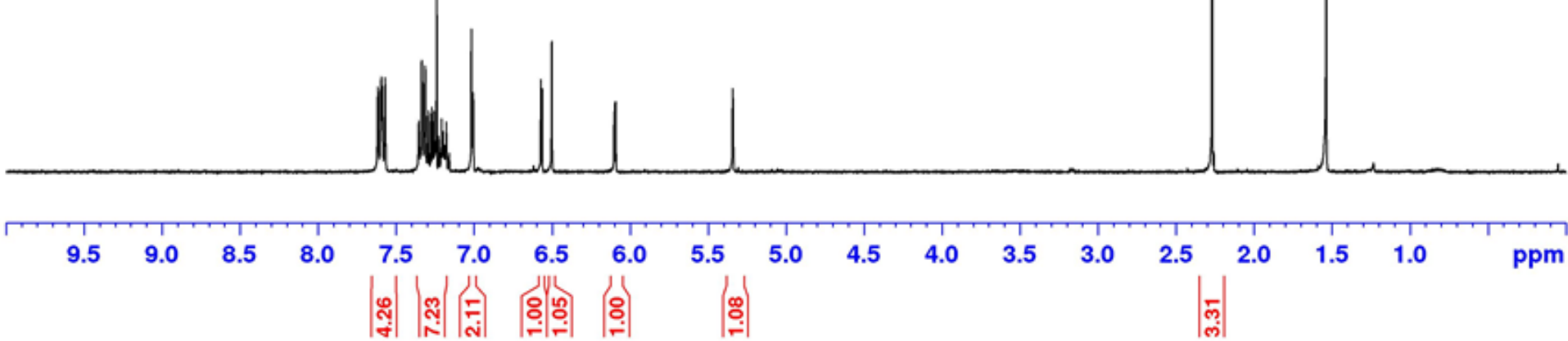


Solvent: $\mathrm{CDCl}_{3}$

SFO1: $150 \mathrm{MHz}$

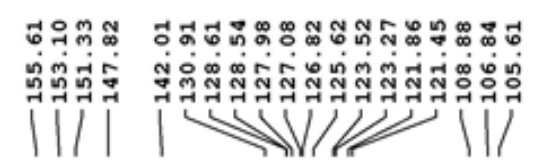

RDK-02-206

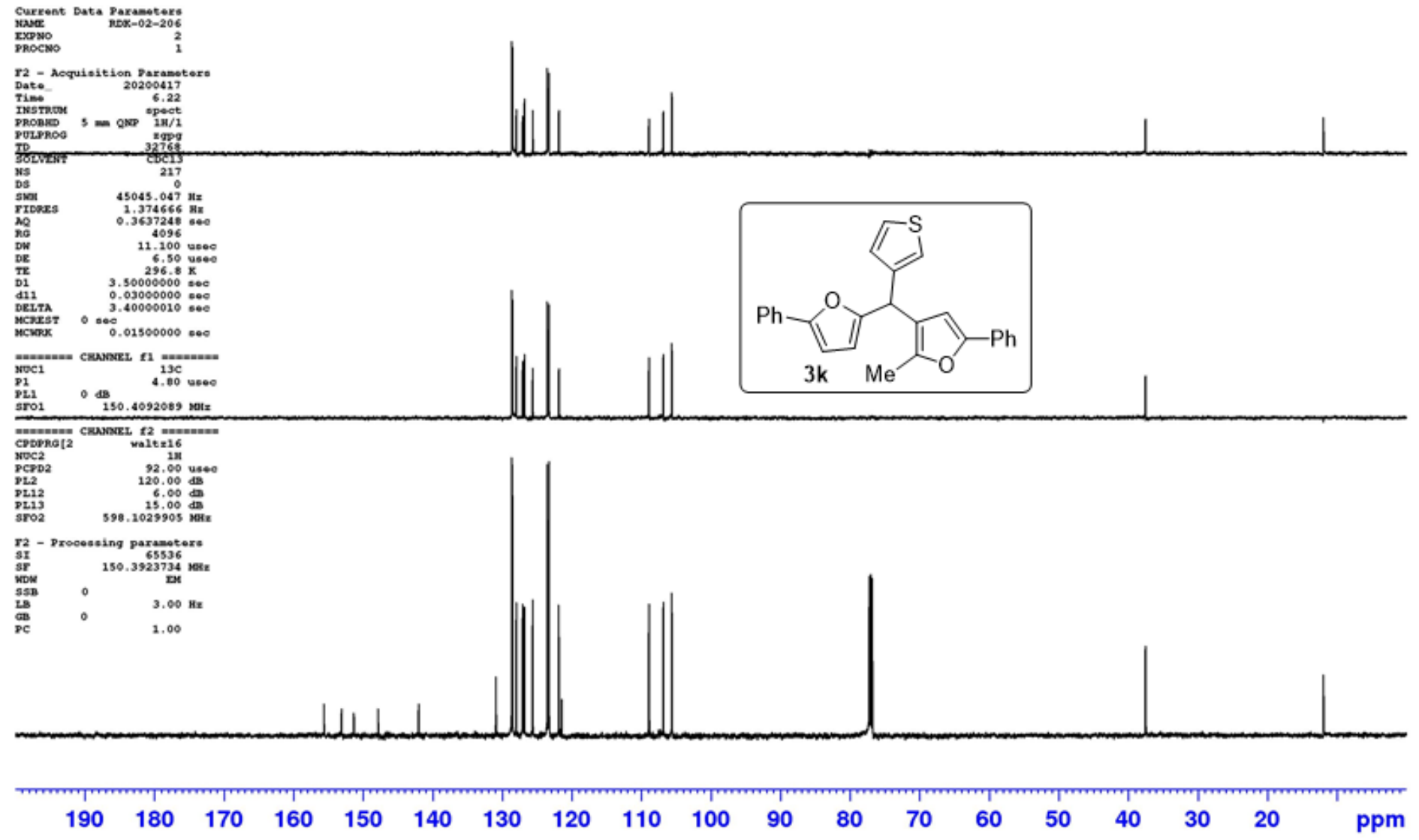


Solvent: $\mathrm{CDCl}_{3}$

SFO1: $600 \mathrm{MHz}$

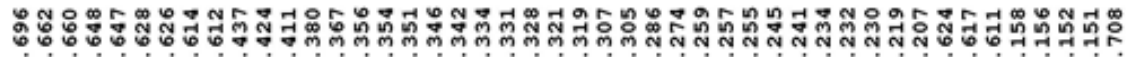

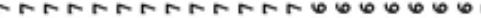

(a)

RDK-02-204

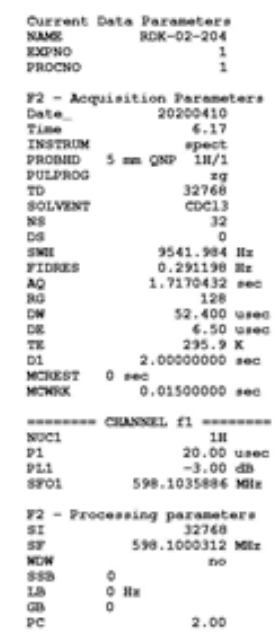

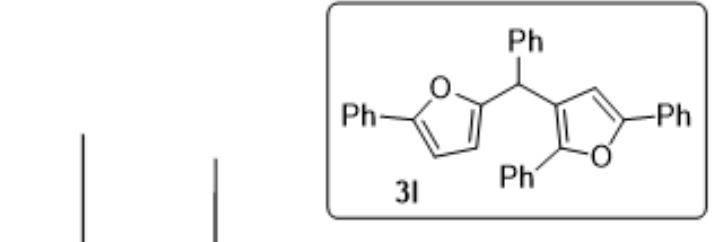

$\begin{array}{llll}9.5 & 9.0 & 8.5 & 8.0\end{array}$ 7.0 เి - 든 全 
Solvent: $\mathrm{CDCl}_{3}$ SFO1: $150 \mathrm{MHz}$
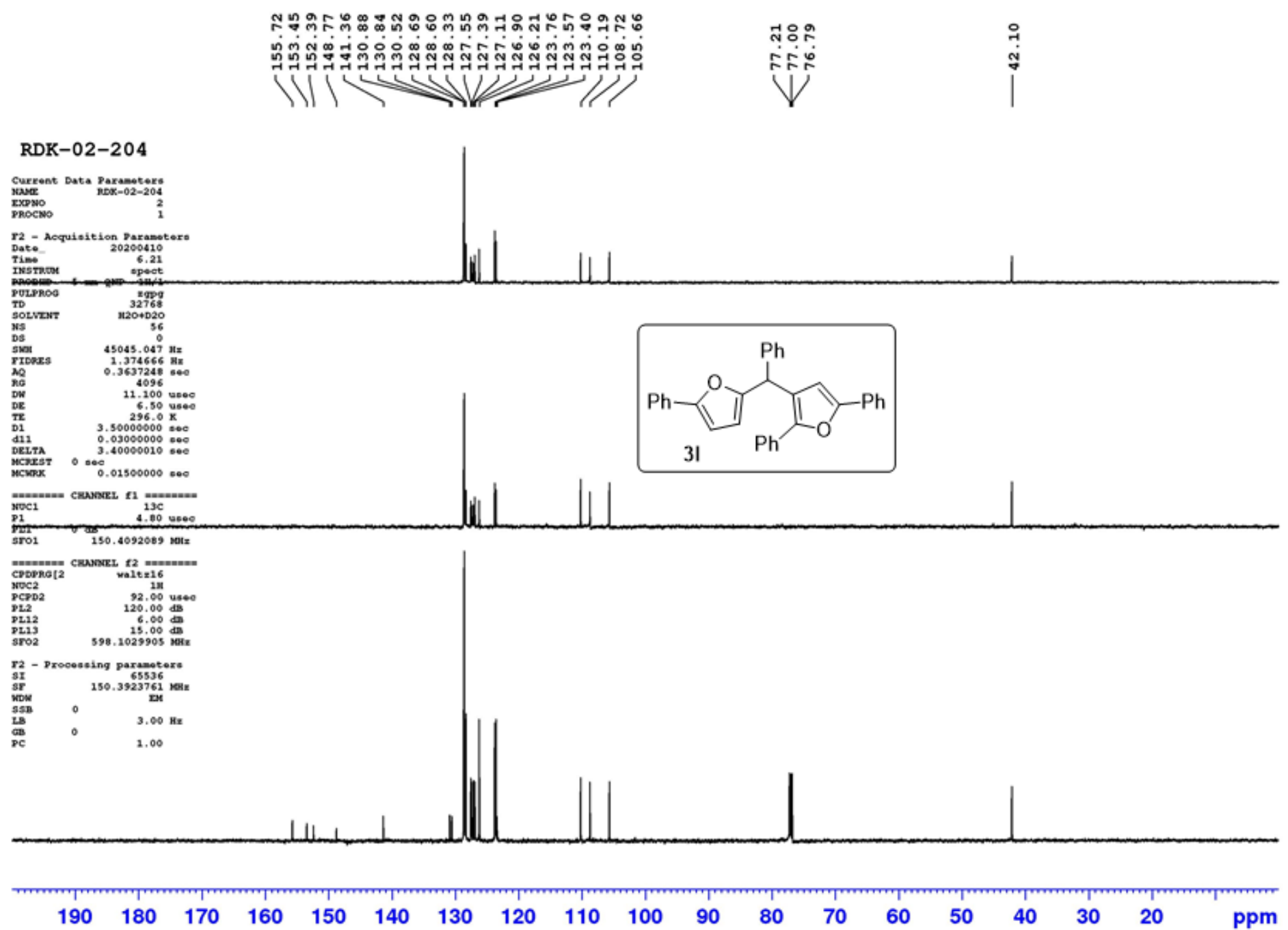
Solvent: $\mathrm{CDCl}_{3}$

SFO1: $400 \mathrm{MHz}$

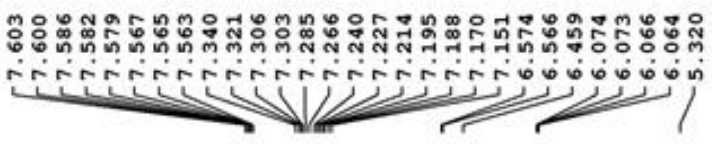

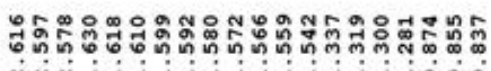

Nल N न

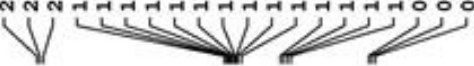

RDK-02-203-P

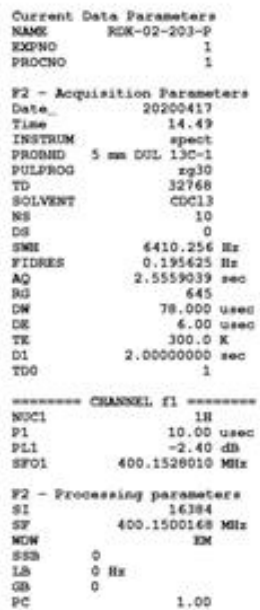

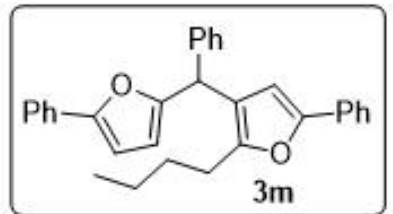

PC 1.00

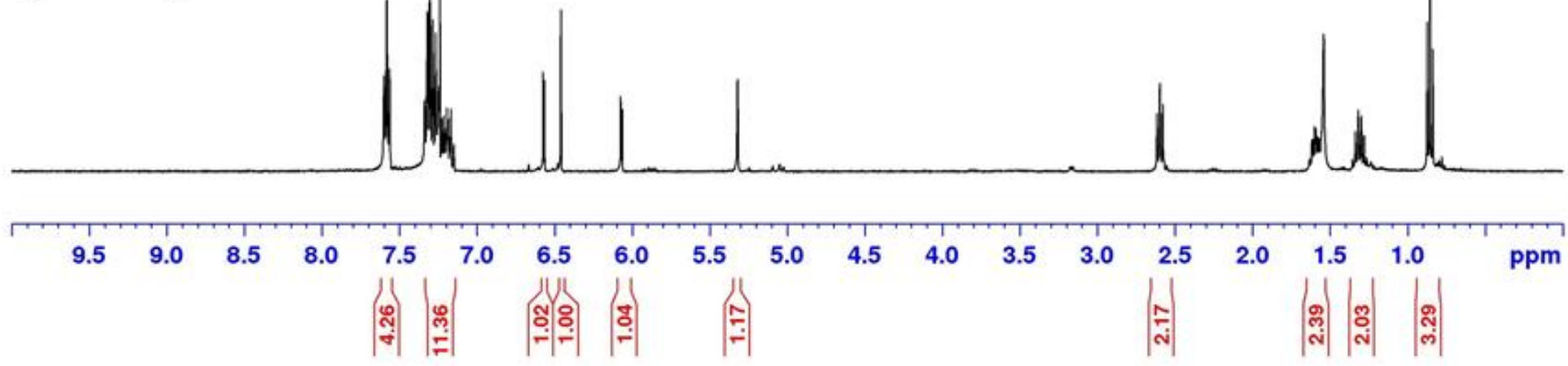


Solvent: $\mathrm{CDCl}_{3}$

SFO1: $150 \mathrm{MHz}$

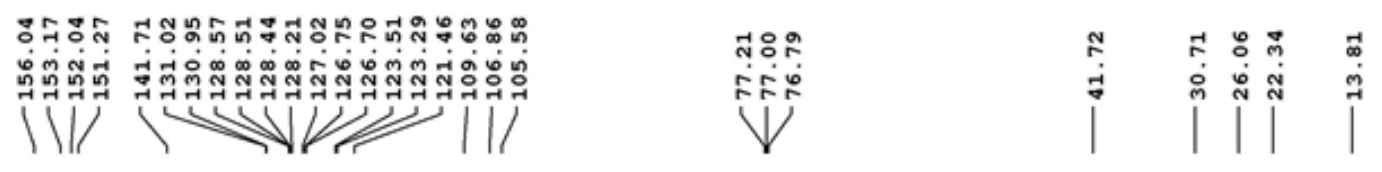

\section{RDK-02-203}

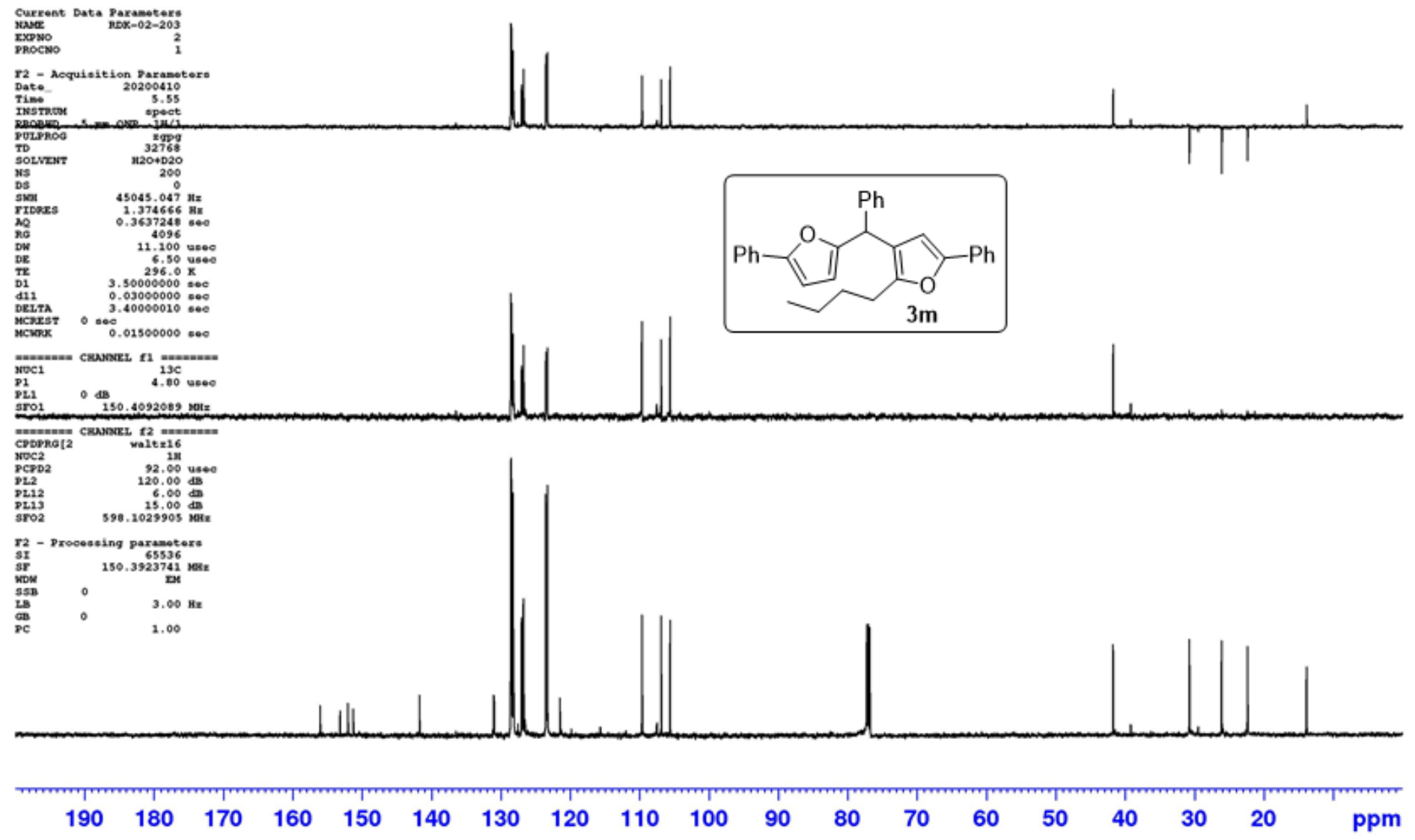


Solvent: $\mathrm{CDCl}_{3}$

SFO1: $600 \mathrm{MHz}$
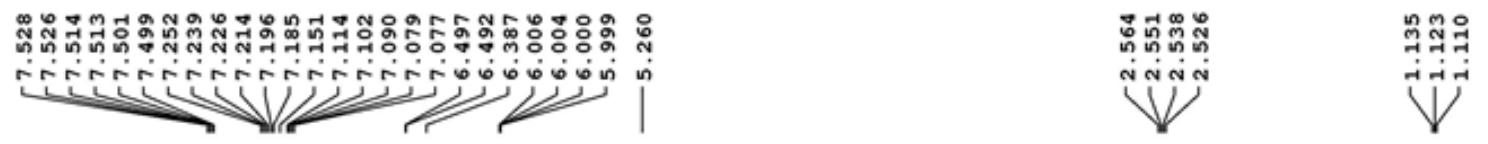

RDK-02-238
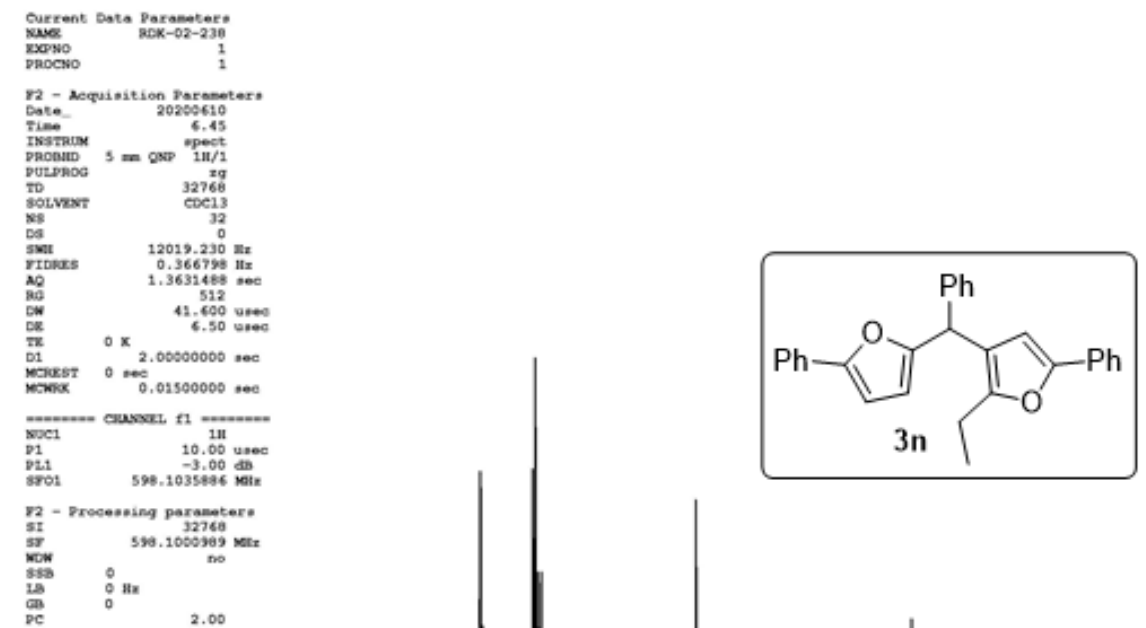
Solvent: $\mathrm{CDCl}_{3}$

SFO1: $150 \mathrm{MHz}$

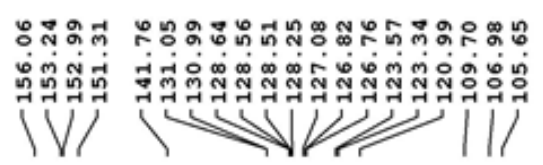

กั้อำ

सF⿱

V

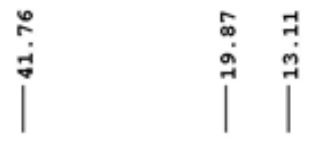

RDK-02-238

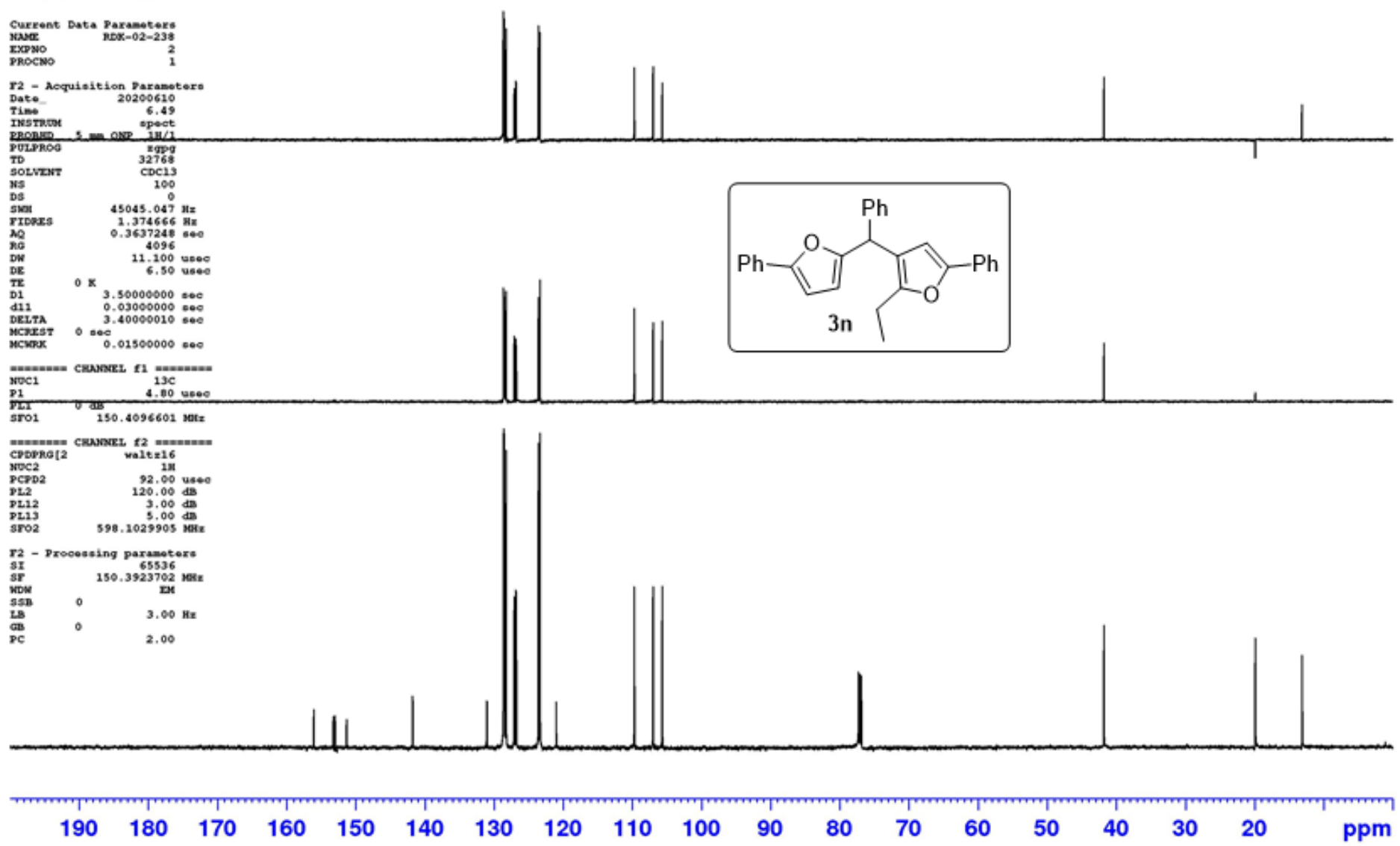


Solvent: $\mathrm{CDCl}_{3}$

SFO1: $400 \mathrm{MHz}$

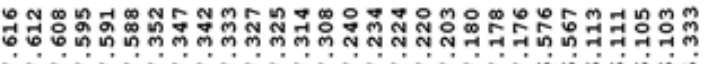

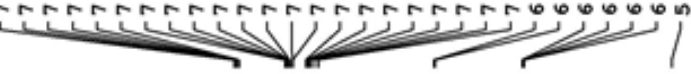

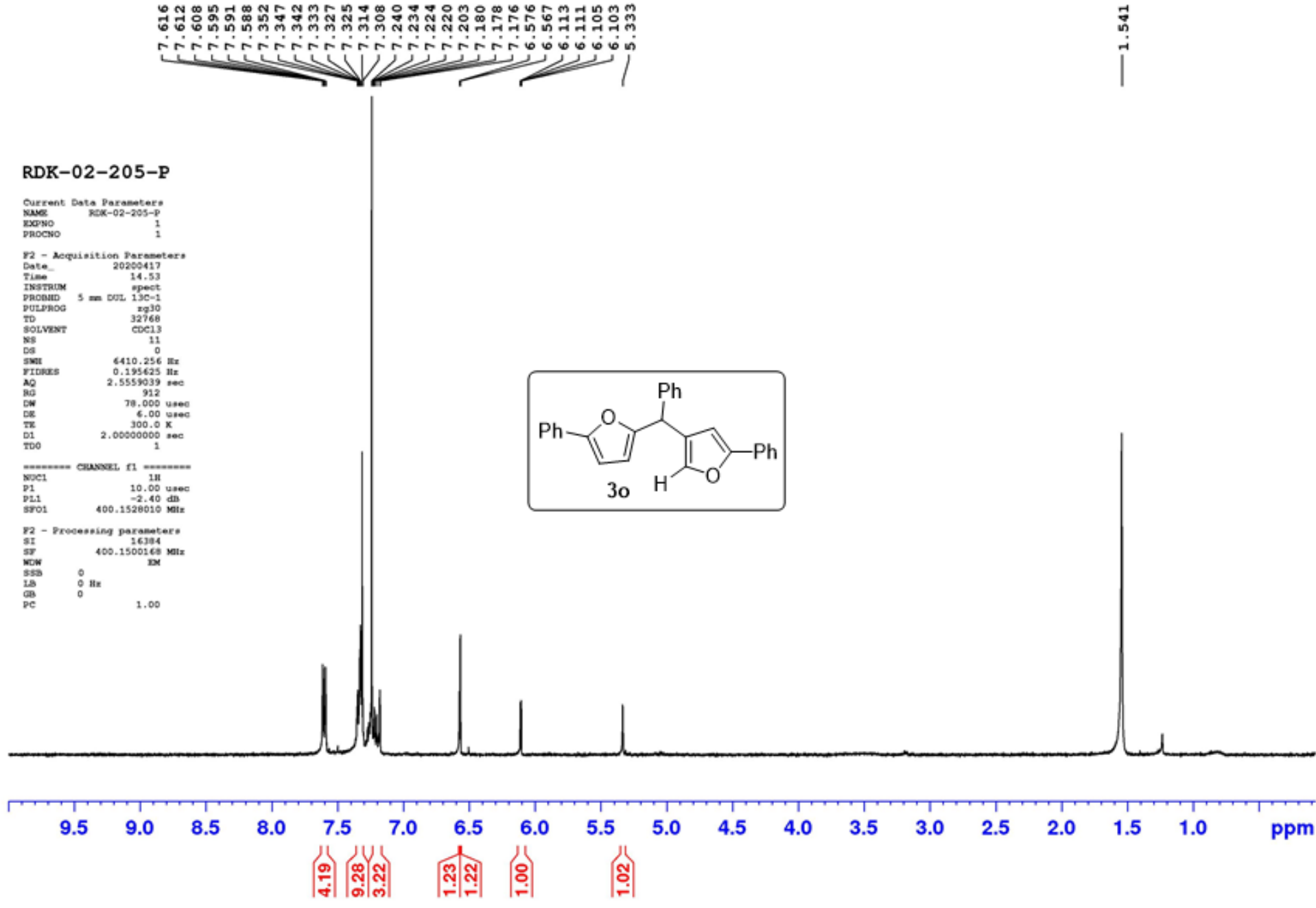


Solvent: $\mathrm{CDCl}_{3}$

SFO1: $150 \mathrm{MHz}$
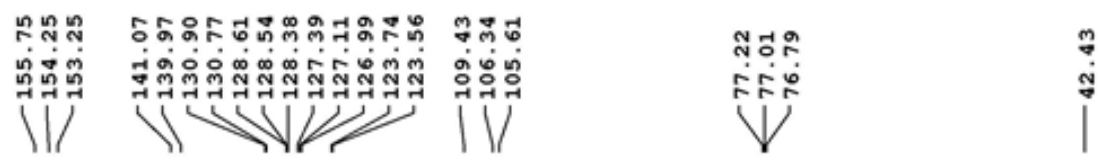

RDK-02-205

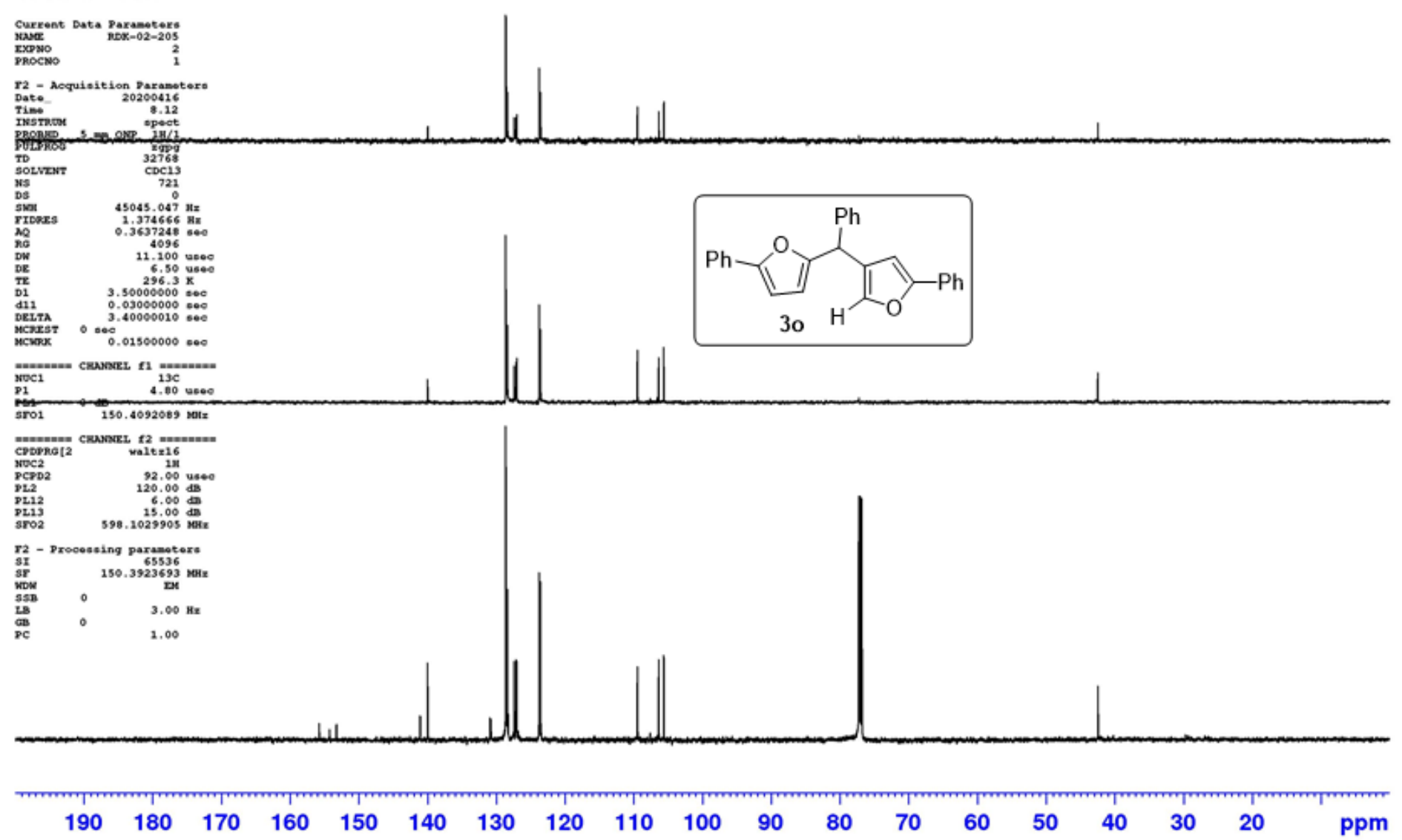


Solvent: $\mathrm{CDCl}_{3}$

SFO1: $600 \mathrm{MHz}$

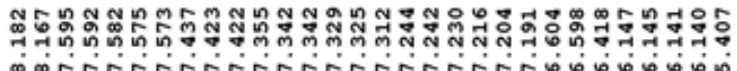

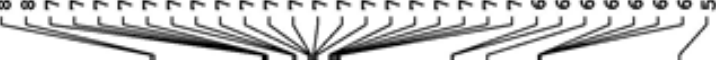

RDK-02-54

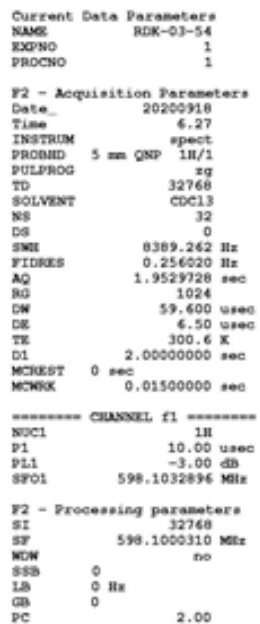

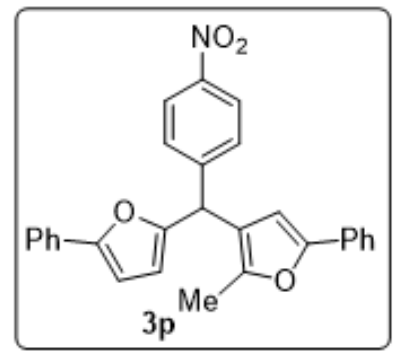

$\perp \mid$
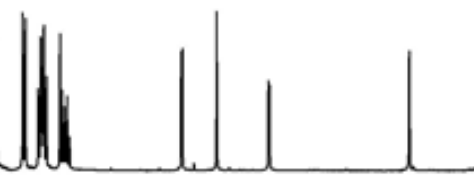

9.5

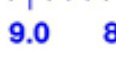

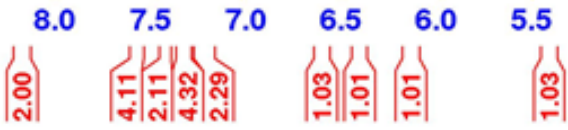

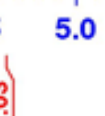

4.0
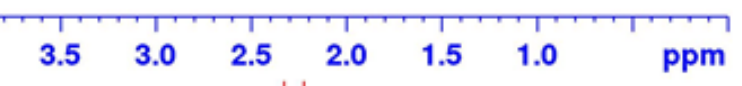

a) 
Solvent: $\mathrm{CDCl}_{3}$

SFO1: $150 \mathrm{MHz}$

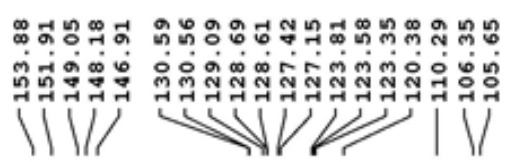

สำำ

सरํำ

V

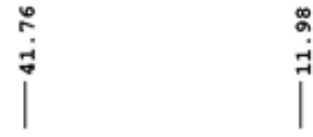

RDK-03-54-C13

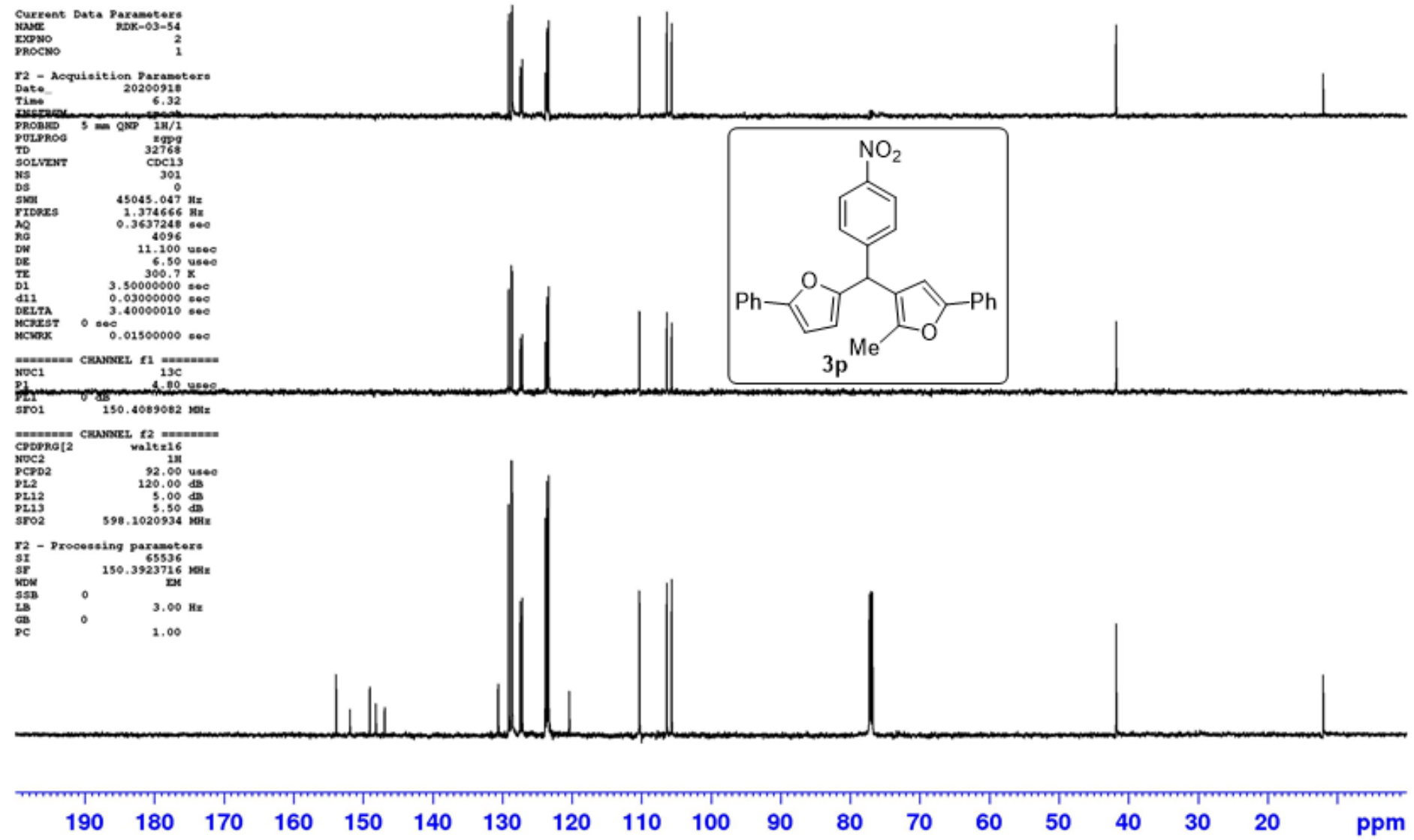


Solvent: $\mathrm{CDCl}_{3}$

SFO1: $400 \mathrm{MHz}$

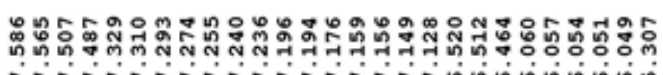

intinting

Ij

RDK-02-216
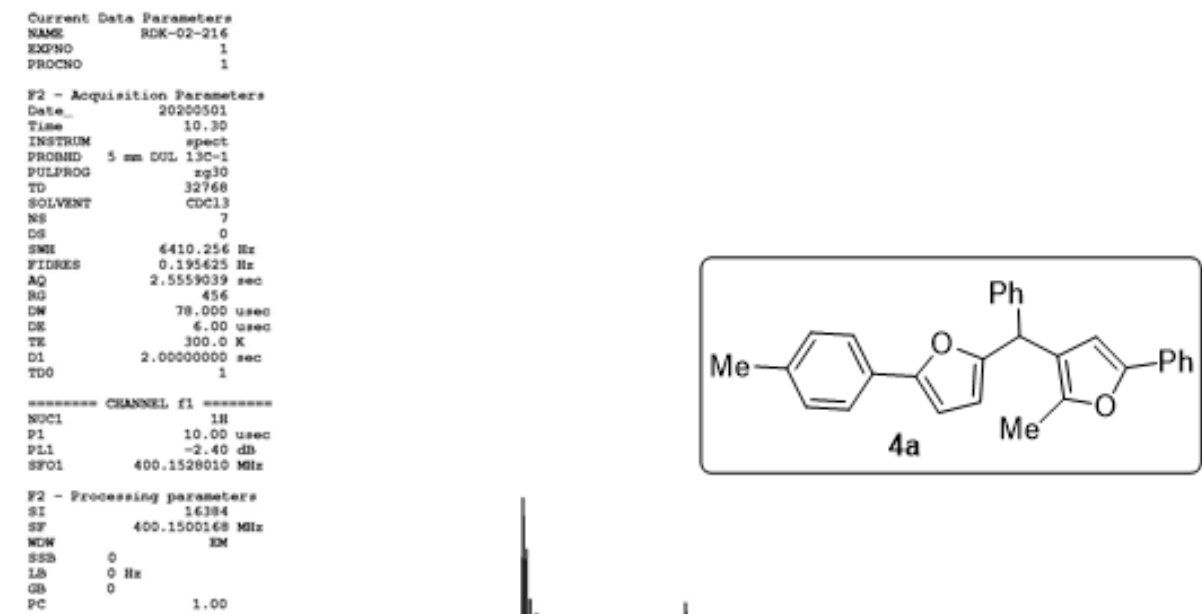

PC 1.00

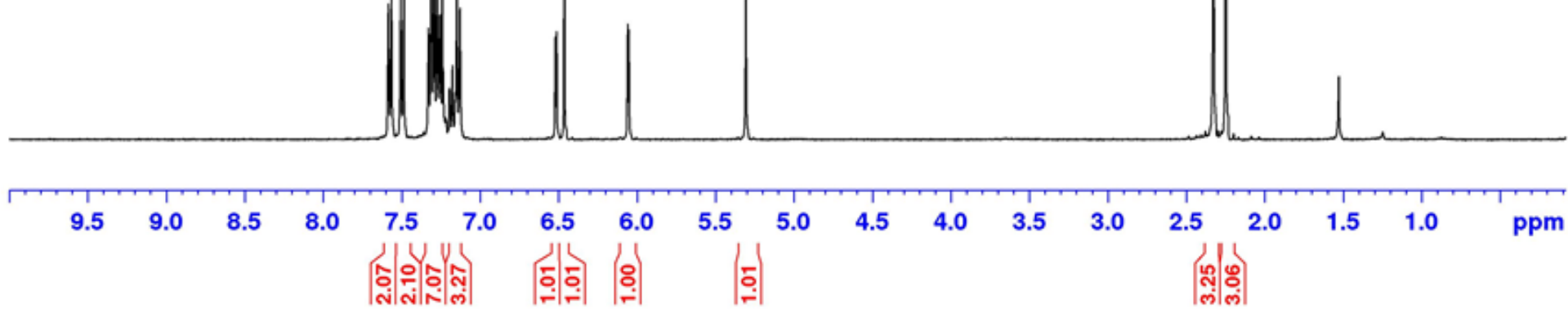


Solvent: $\mathrm{CDCl}_{3}$ SFO1: $100 \mathrm{MHz}$
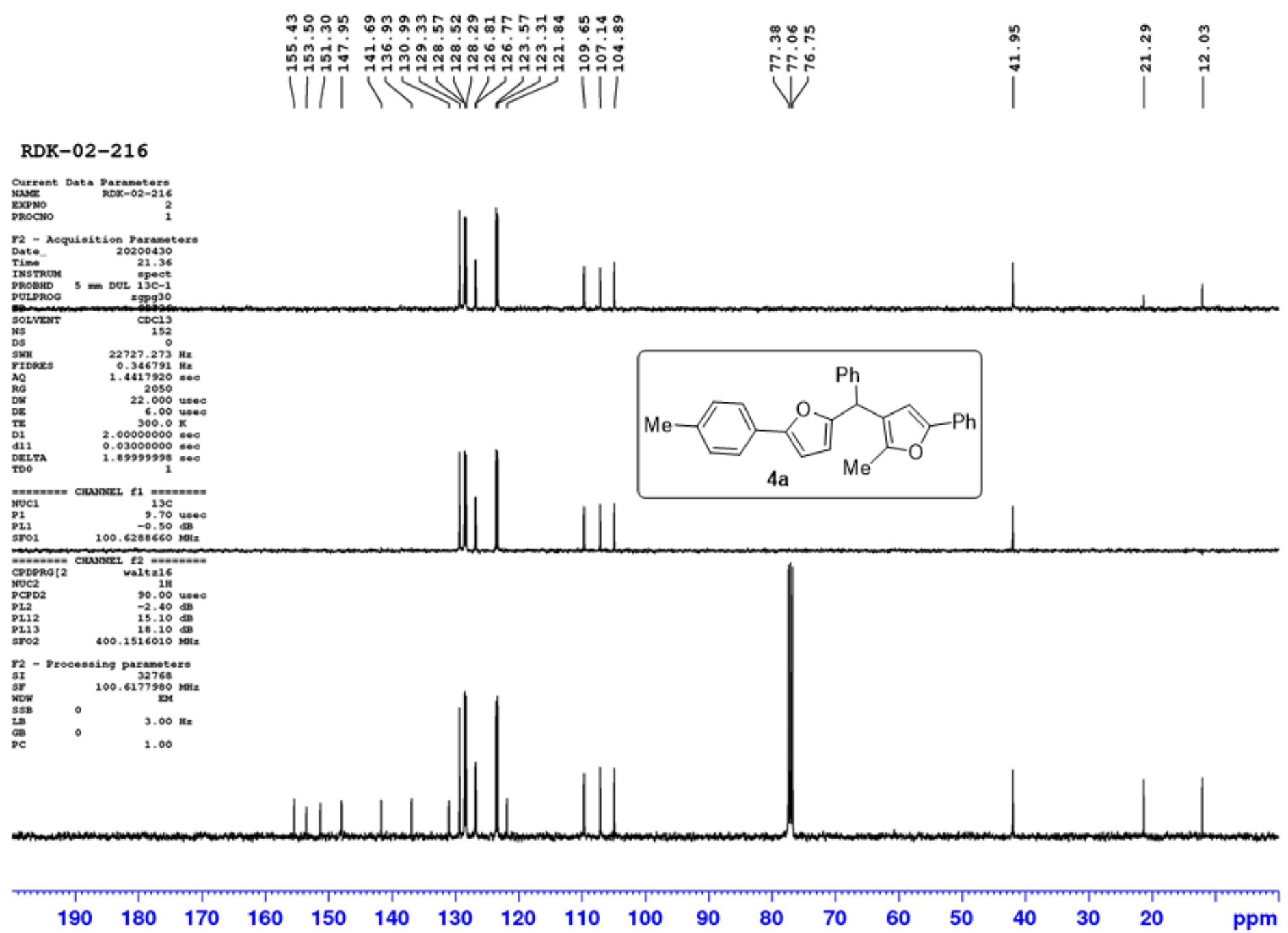
Solvent: $\mathrm{CDCl}_{3}$

SFO1: $600 \mathrm{MHz}$

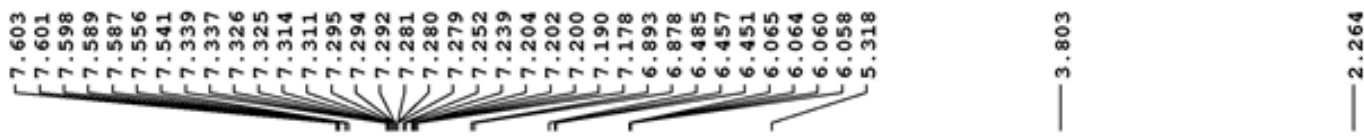

RDK-02-217

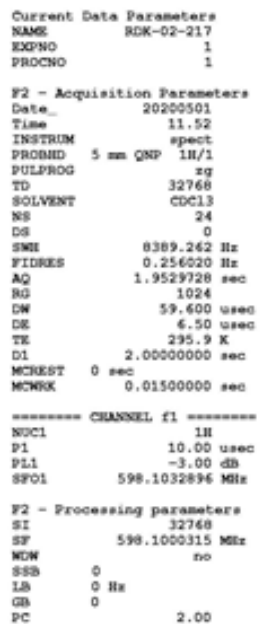

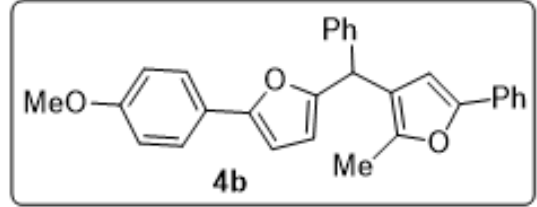

?ִ

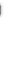

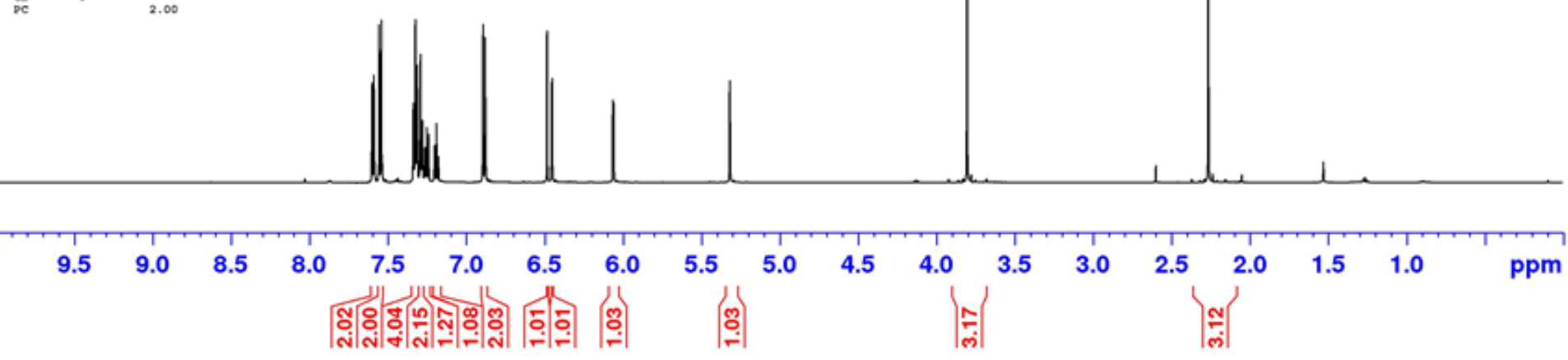


Solvent: $\mathrm{CDCl}_{3}$ SFO1: $150 \mathrm{MHz}$
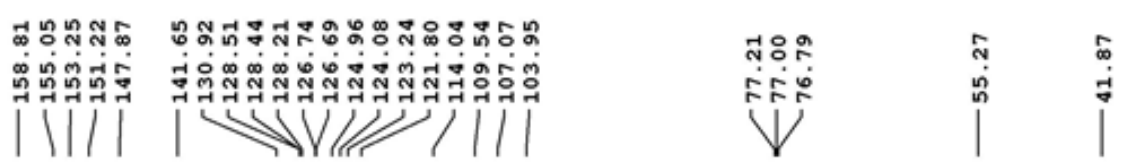

\%

RDK-02-217

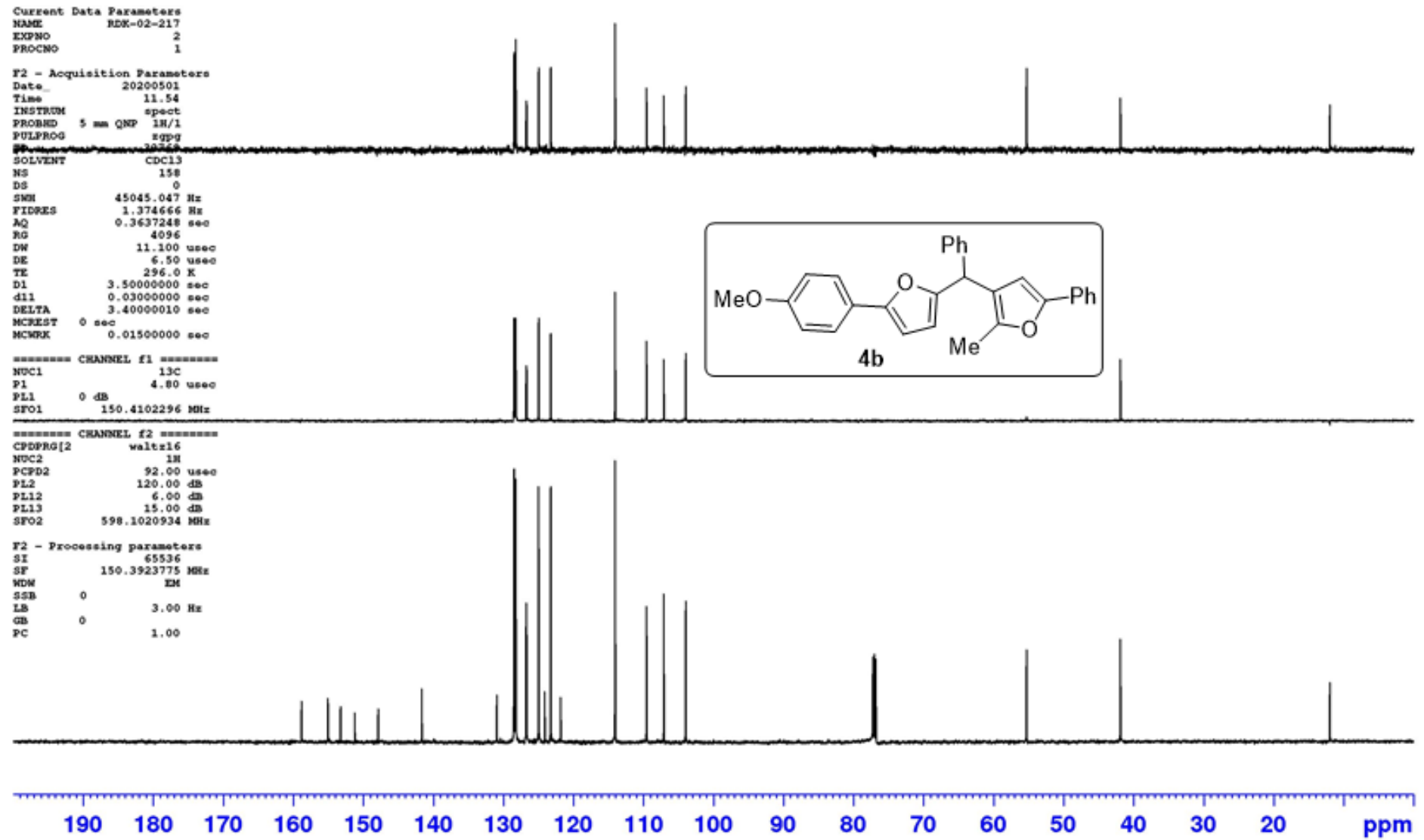


Solvent: $\mathrm{CDCl}_{3}$

SFO1: $600 \mathrm{MHz}$
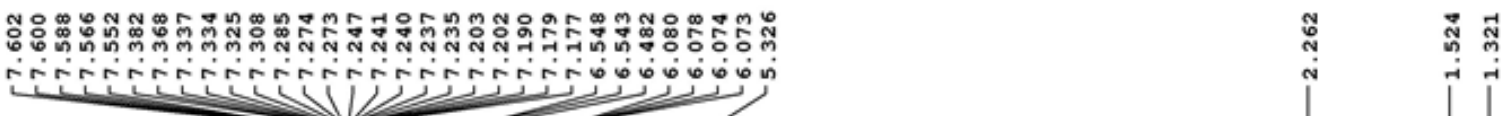

ank

RDK-02-234

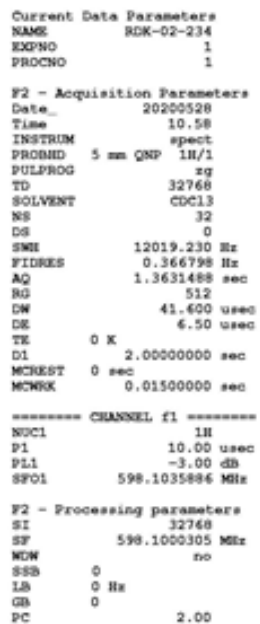
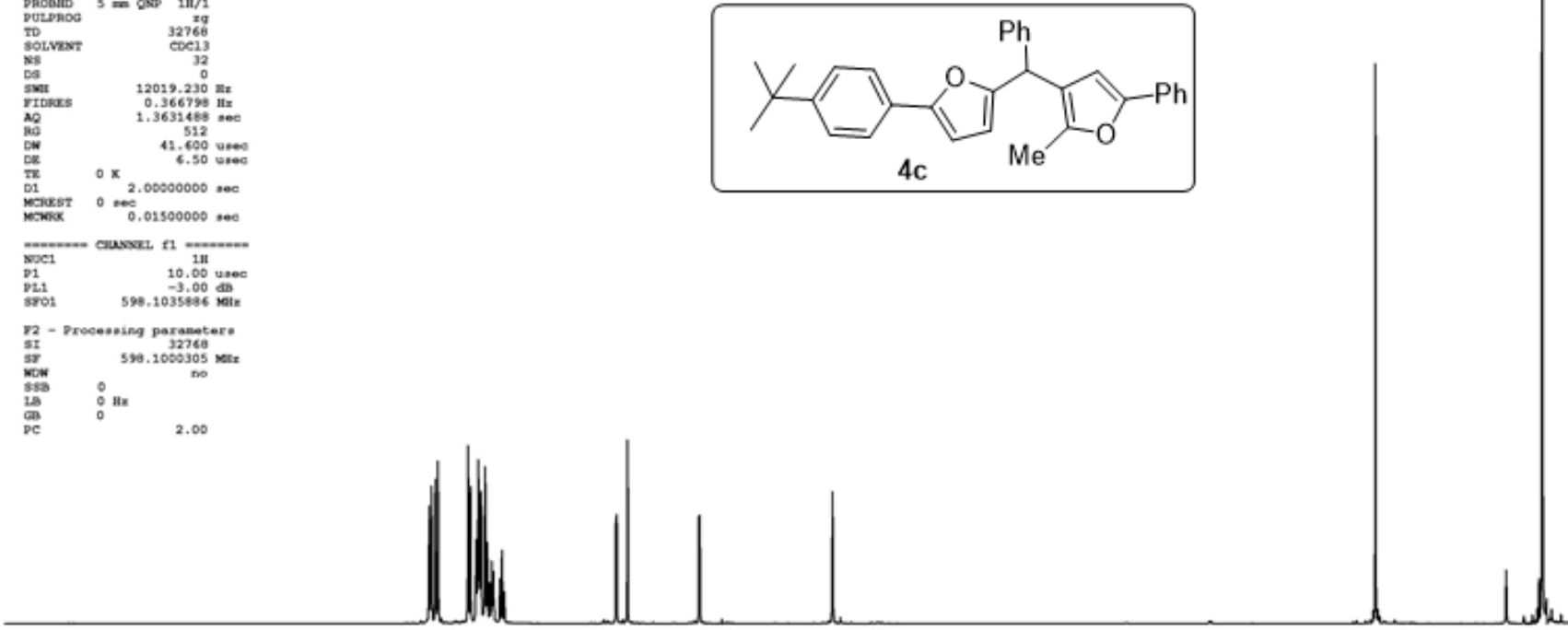

$\begin{array}{lll}9.5 & 9.0 & 8.5\end{array}$

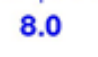

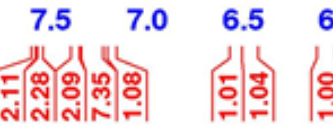

5.5

5.04 .5

$\begin{array}{llll}5 & 4.0 & 3.5 & 3.0\end{array}$

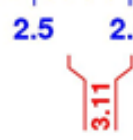

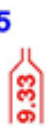


Solvent: $\mathrm{CDCl}_{3}$ SFO1: $150 \mathrm{MHz}$

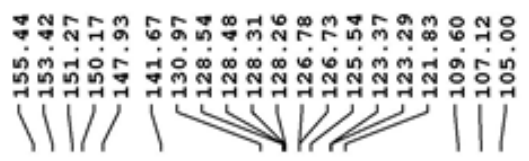

RDK-02-234

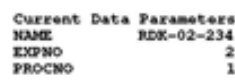

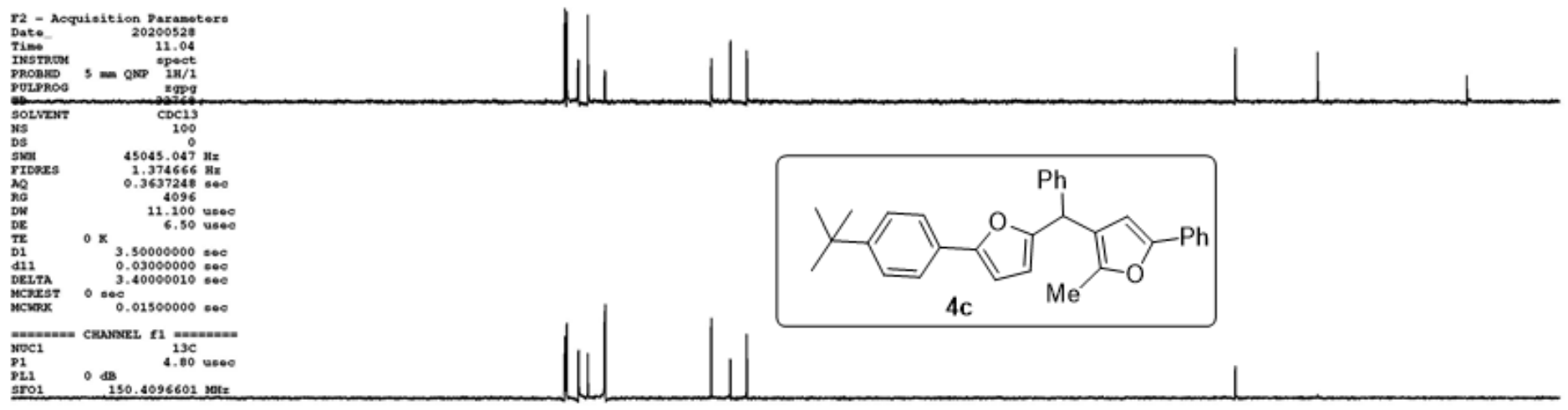

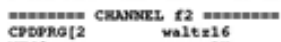

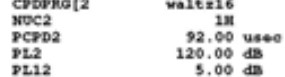

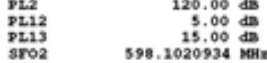

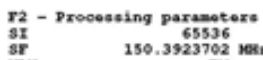

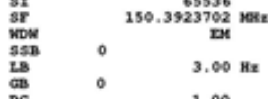

PC $\quad 1.00$

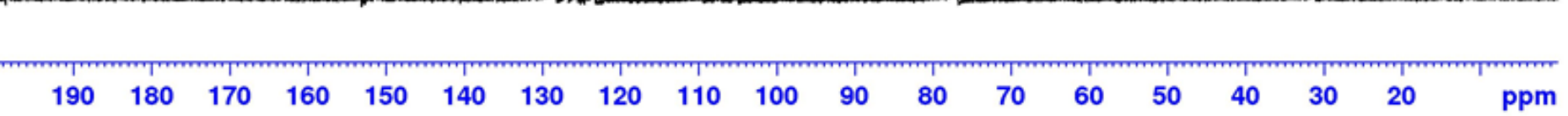


Solvent: $\mathrm{CDCl}_{3}$

SFO1: $600 \mathrm{MHz}$

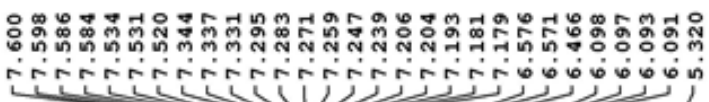

จุ

(N)

RDK-02-212

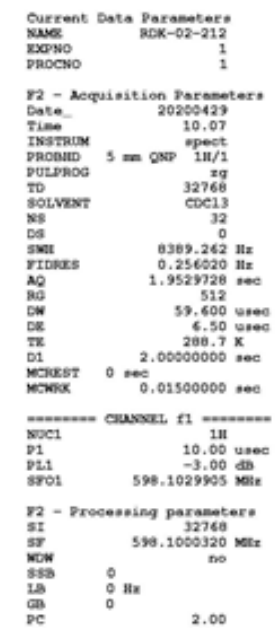

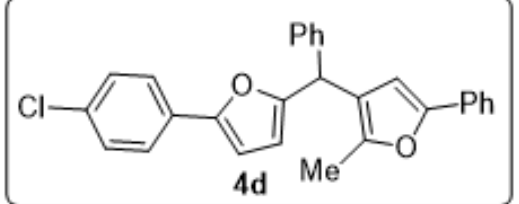

\.
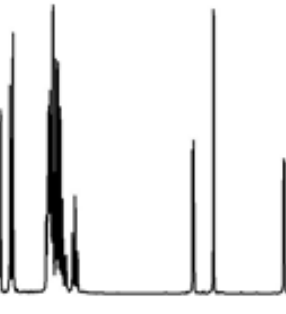

9.5

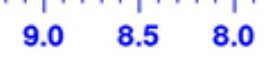

$7.5{ }^{7.0}$

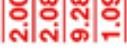

$6.5 \quad 6.0$

$5.5 \quad 5.0$

!)

$4.5 \quad 4.0$

03

3.0

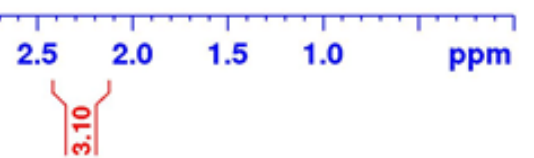


Solvent: $\mathrm{CDCl}_{3}$ SFO1: $150 \mathrm{MHz}$
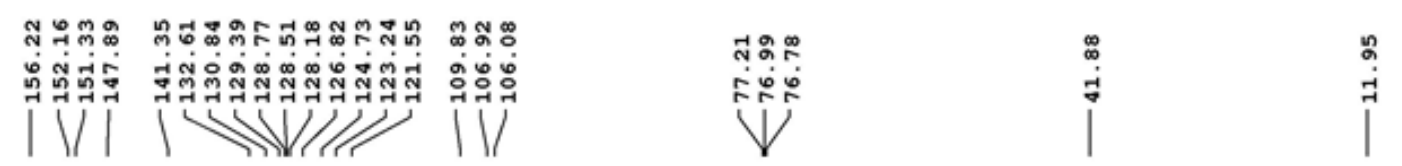

\section{RDK-02-212}

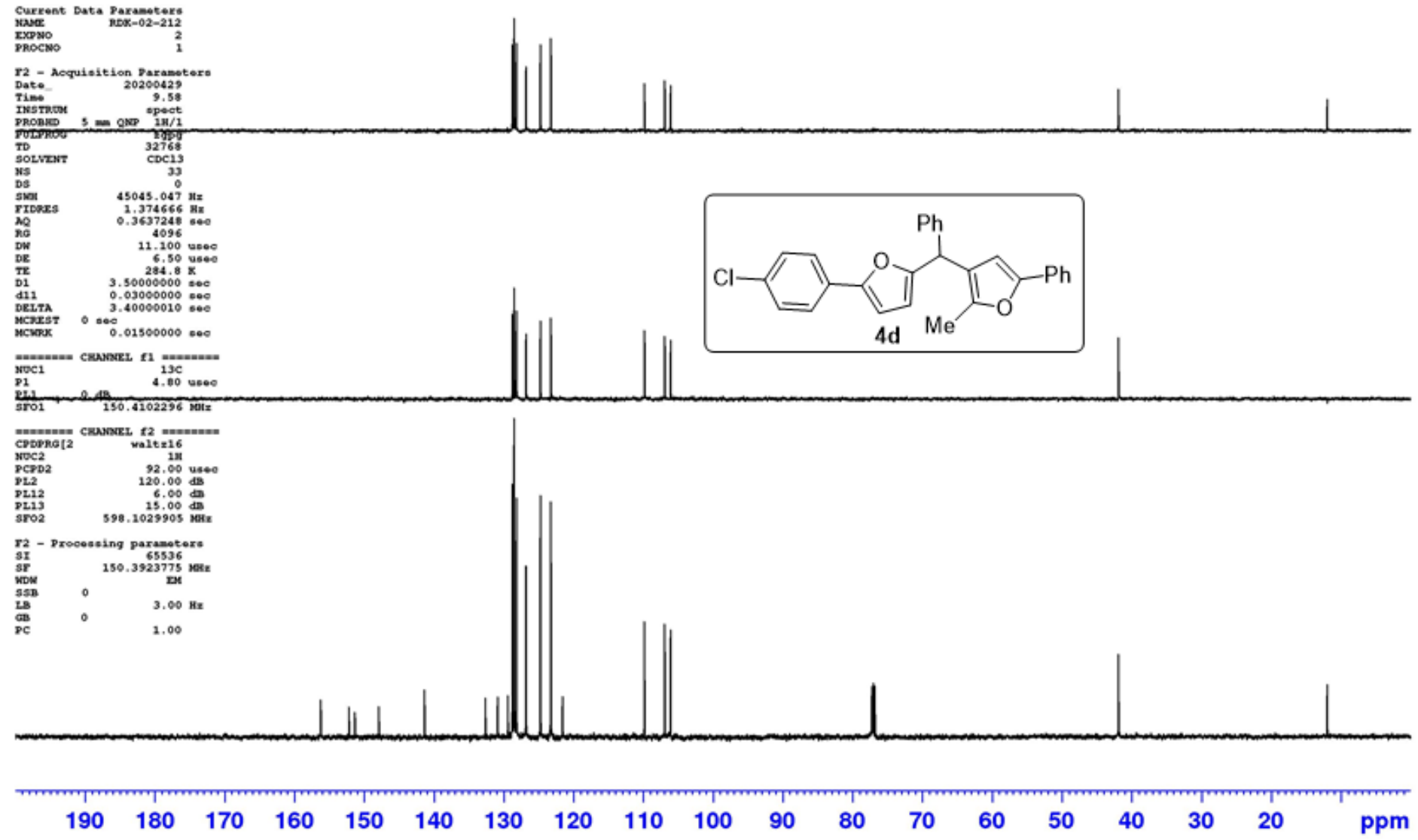


Solvent: $\mathrm{CDCl}_{3}$

SFO1: $600 \mathrm{MHz}$

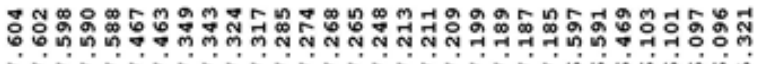

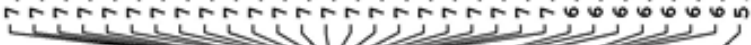

L

RDK-02-209
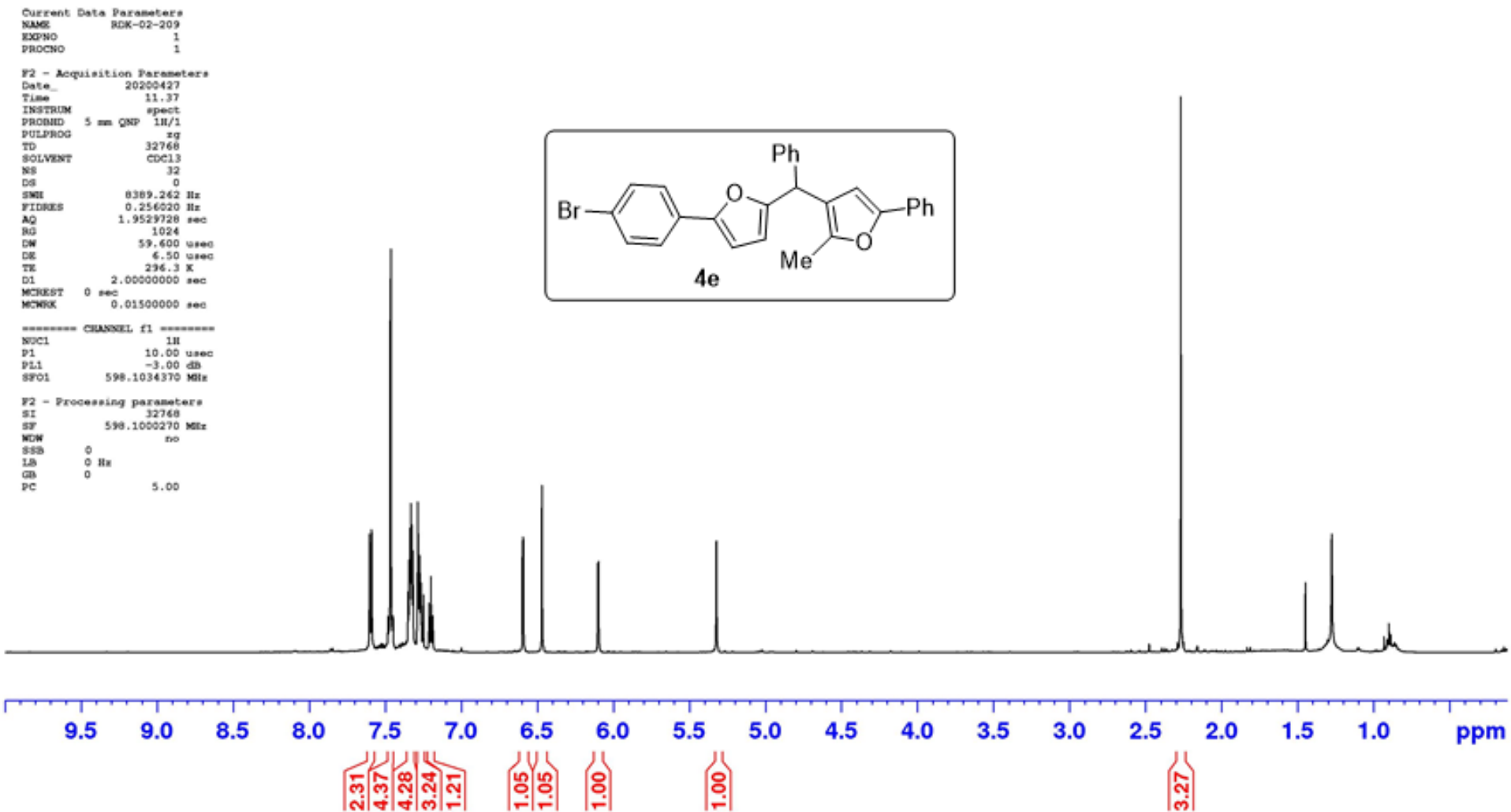
Solvent: $\mathrm{CDCl}_{3}$

SFO1: $150 \mathrm{MHz}$

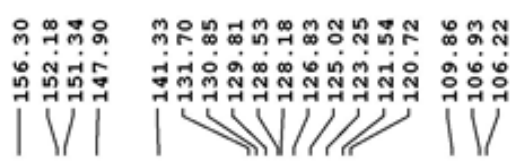

สำำ

당

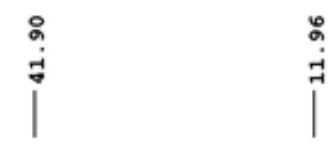

RDK-02-209

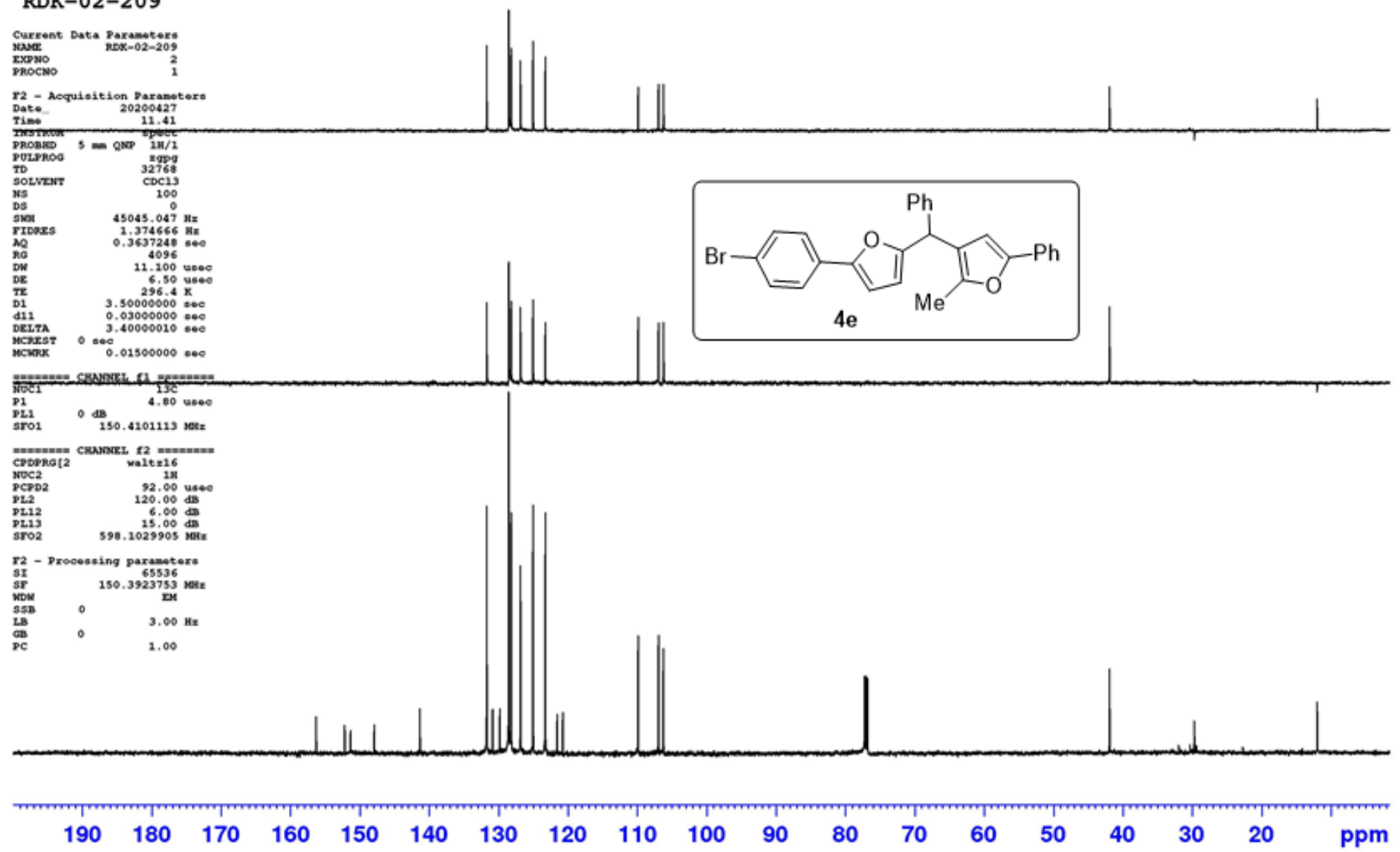


Solvent: $\mathrm{CDCl}_{3}$

SFO1: $600 \mathrm{MHz}$

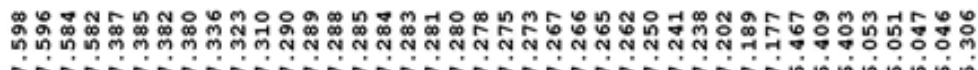

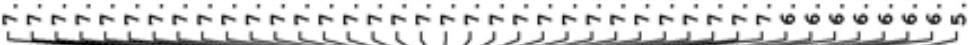

$\longrightarrow$

RDK-02-218

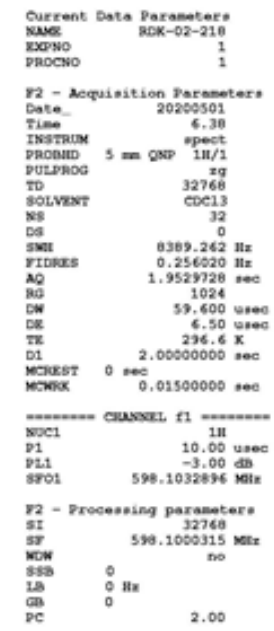

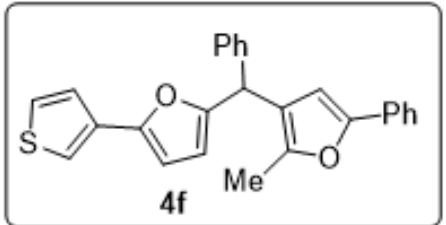

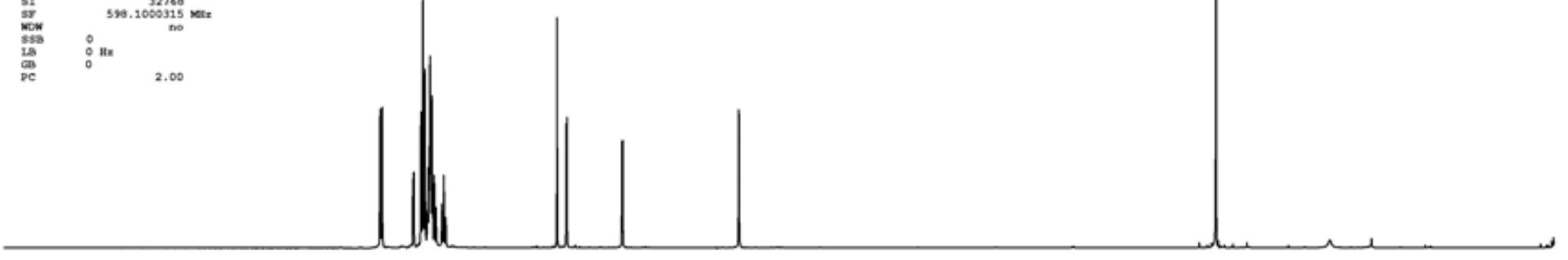

$\begin{array}{llll}9.5 & 9.0 & 8.5 & 8.0\end{array}$

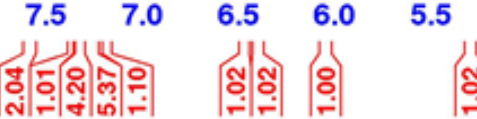

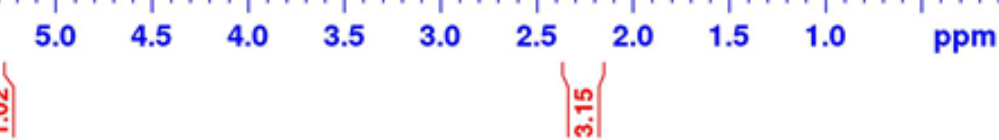


Solvent: $\mathrm{CDCl}_{3}$ SFO1: $150 \mathrm{MHz}$

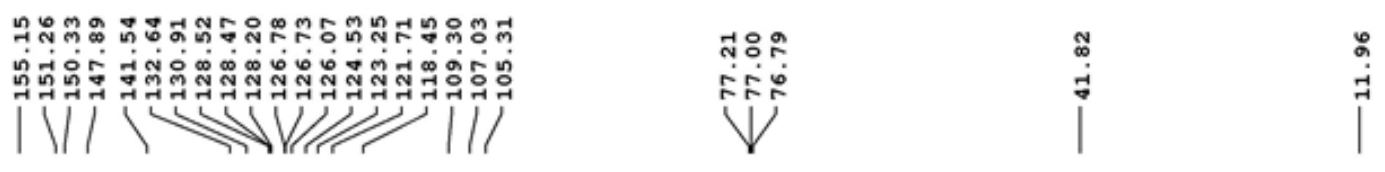

RDK-02-218

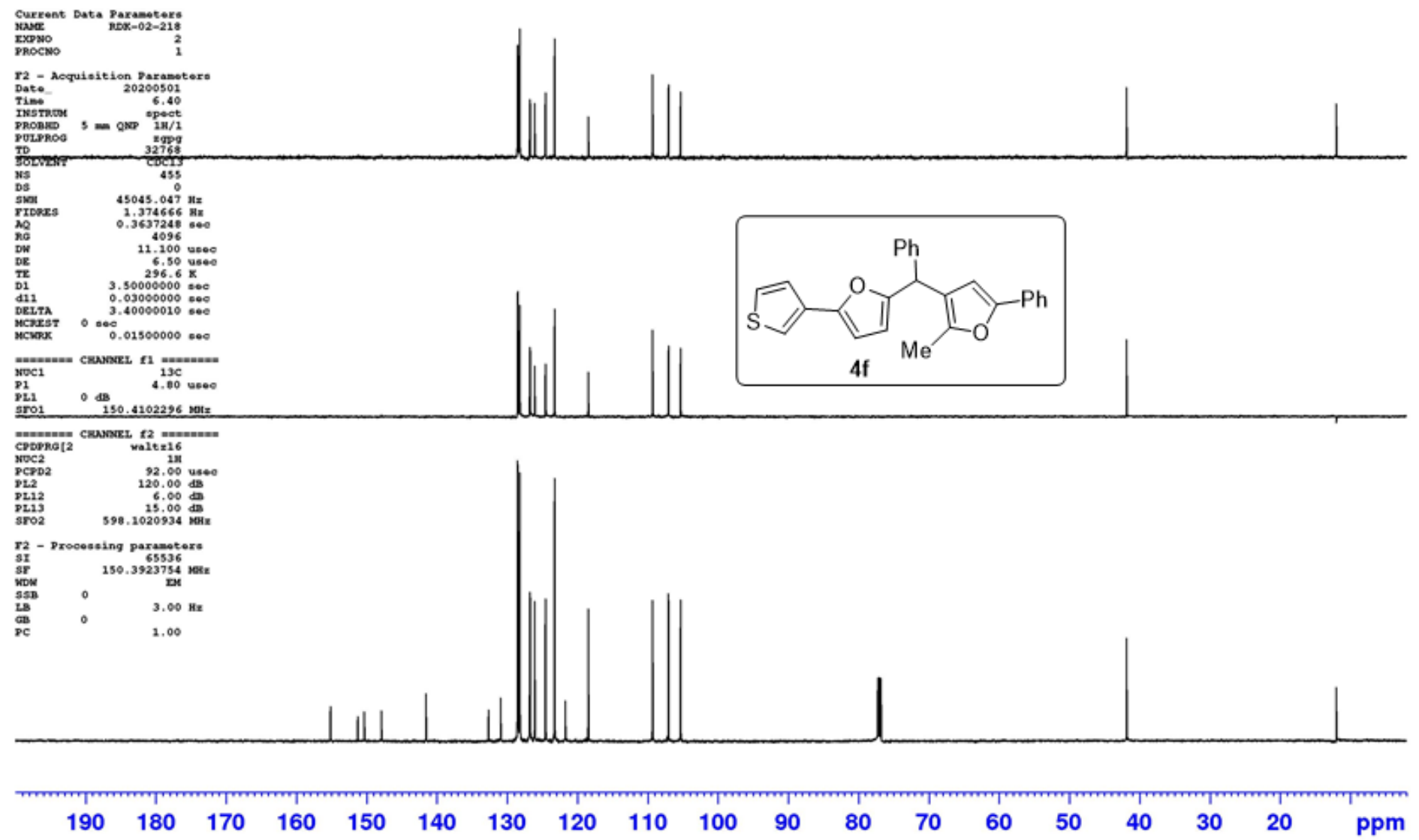


Solvent: $\mathrm{CDCl}_{3}$

SFO1: $600 \mathrm{MHz}$

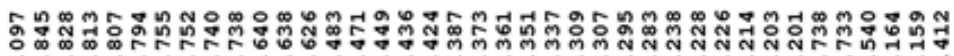

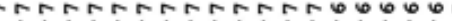

$\longrightarrow+1$

RDK-02-219

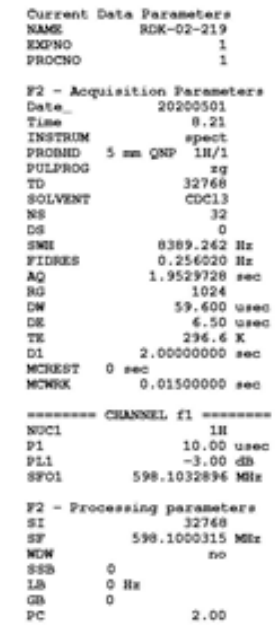

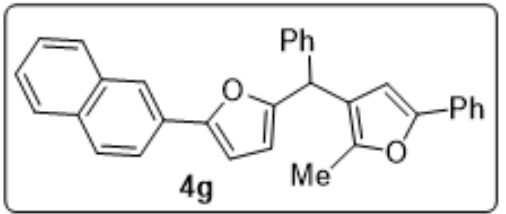

$\mid$

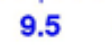

9.0

8.58 .0

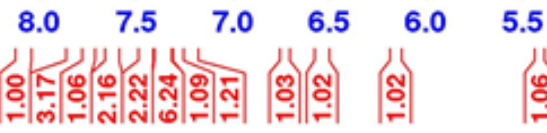

5.0

4.5

4.0

$3.5 \quad 3.0$

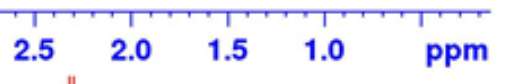

ले. 
Solvent: $\mathrm{CDCl}_{3}$ SFO1: $150 \mathrm{MHz}$

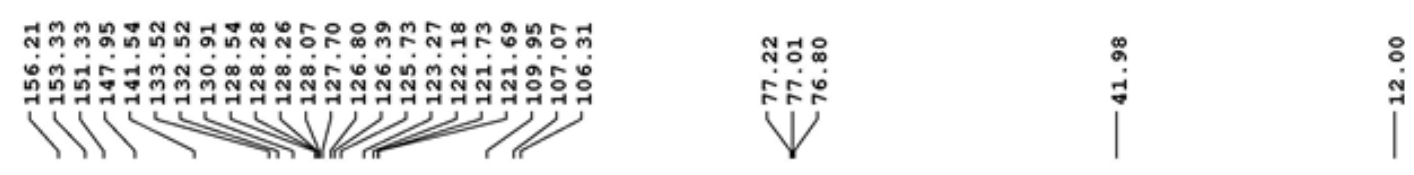

RDK-02-219

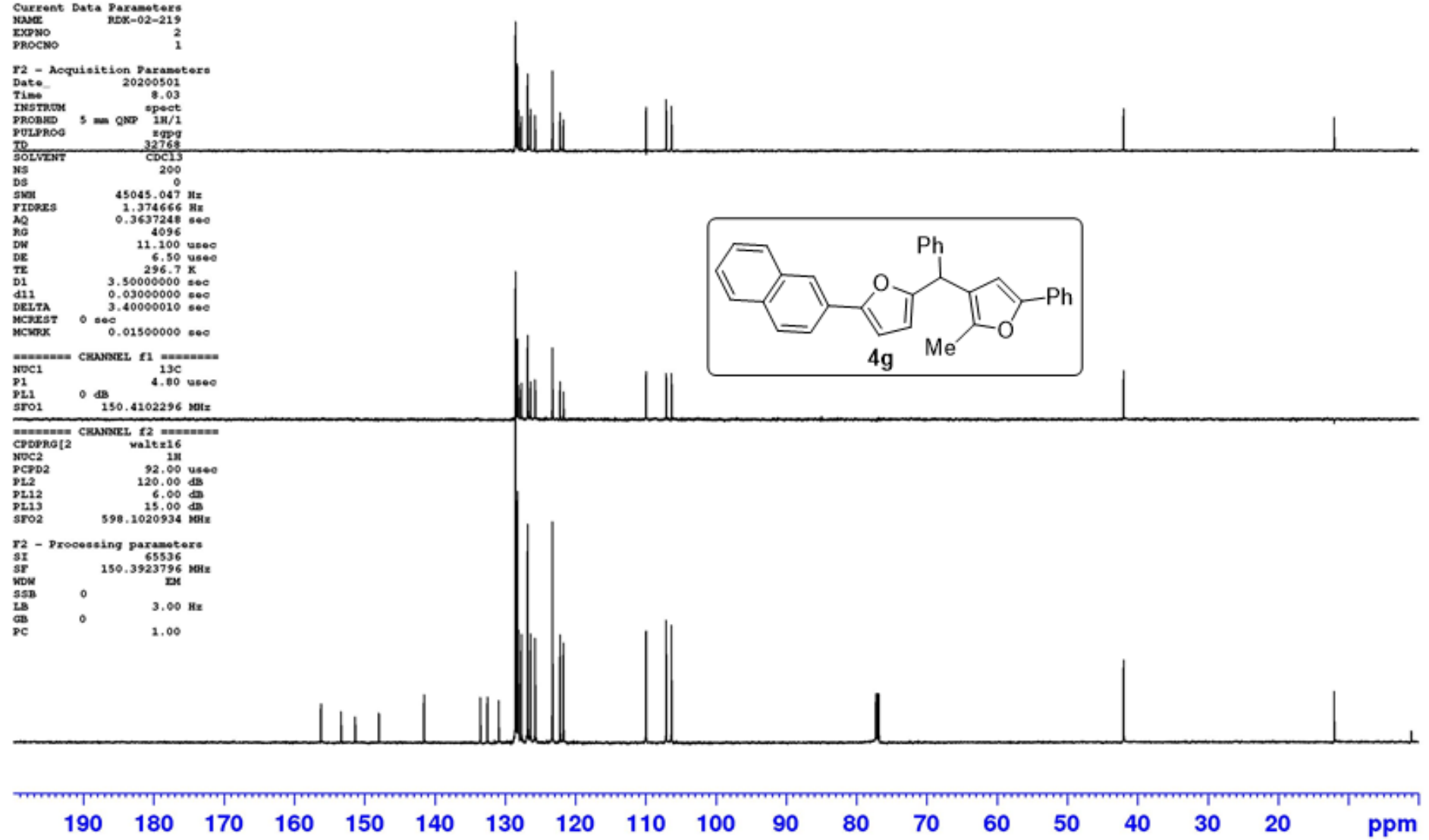


Solvent: $\mathrm{CDCl}_{3}$

SFO1: $400 \mathrm{MHz}$

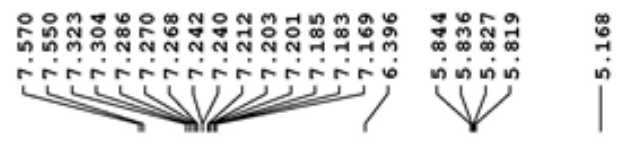

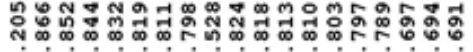

00000

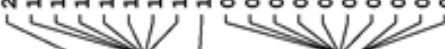

2DK-02-222-F
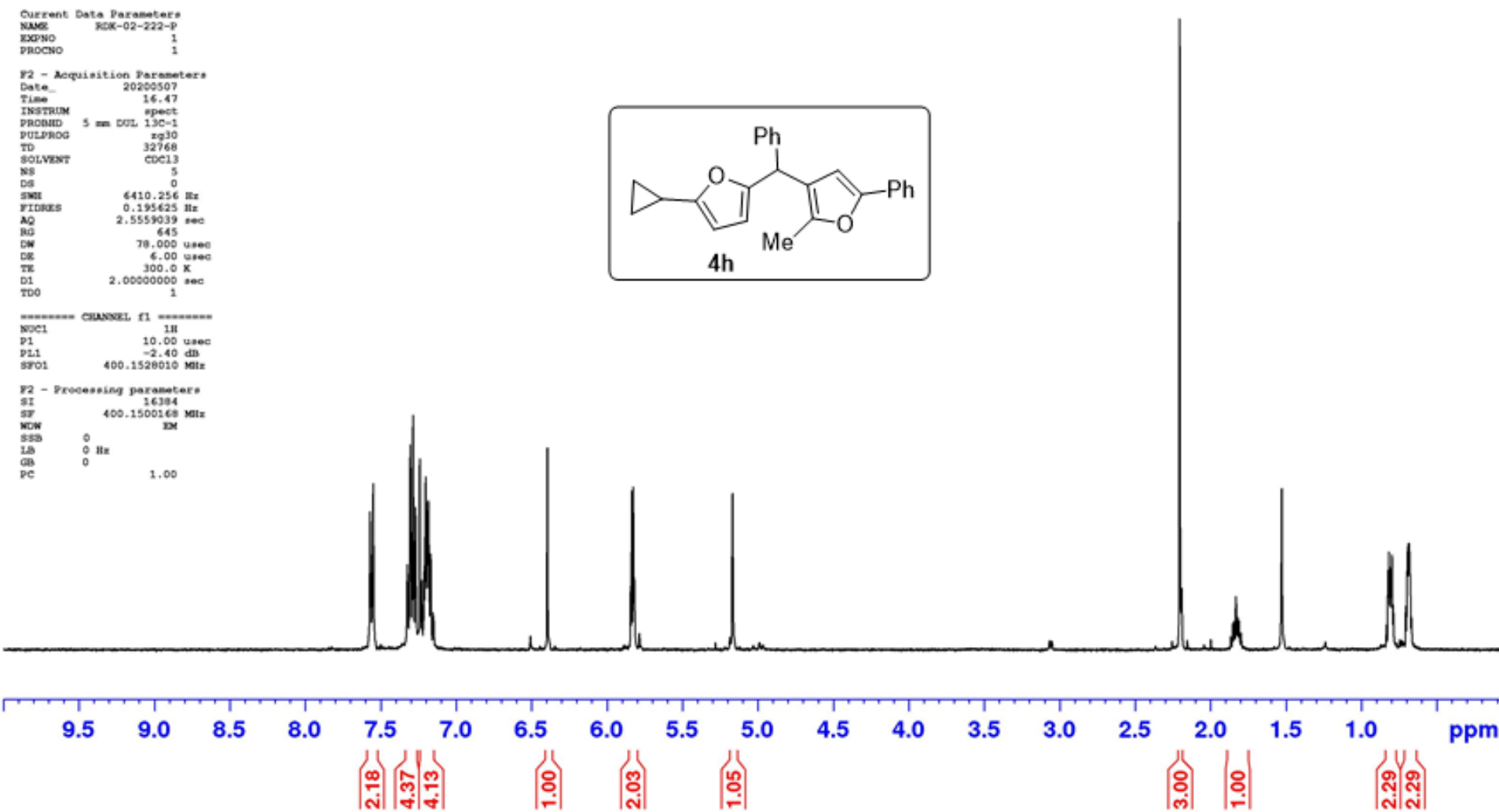
Solvent: $\mathrm{CDCl}_{3}$

SFO1: $150 \mathrm{MHz}$

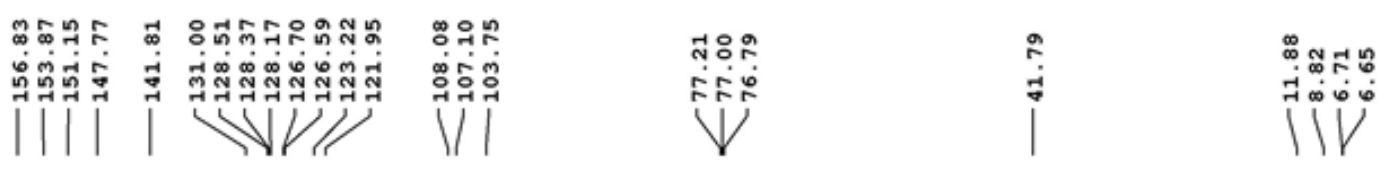

RDK-02-222

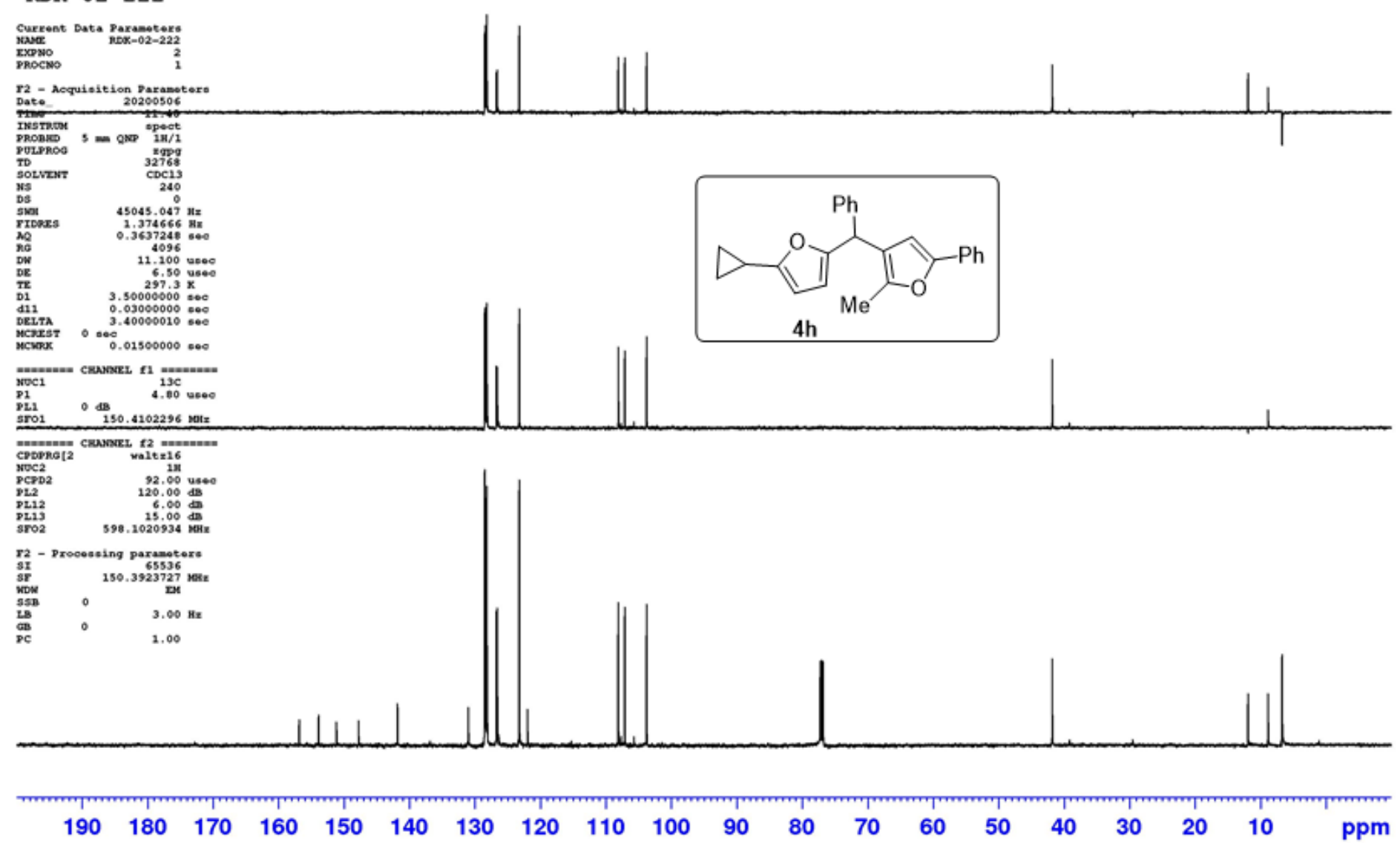


Solvent: $\mathrm{CDCl}_{3}$

SFO1: $600 \mathrm{MHz}$

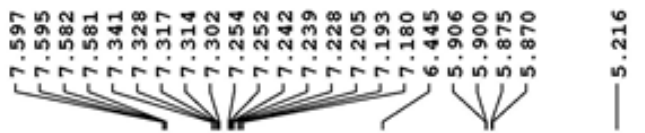

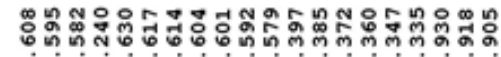

N N N

$\mathrm{V}$

RDK-02-223

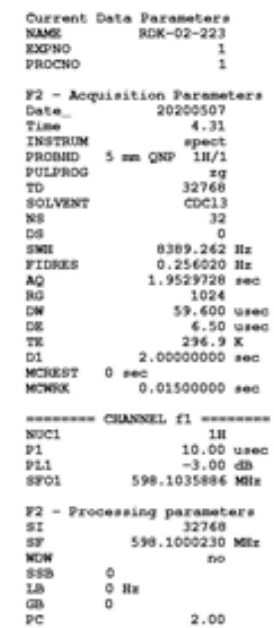

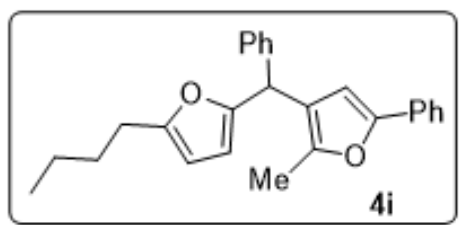

||

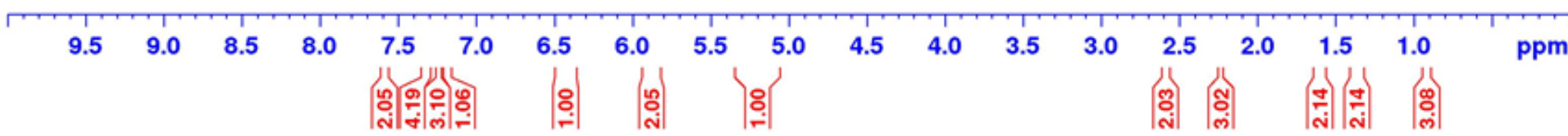


Solvent: $\mathrm{CDCl}_{3}$ SFO1: $150 \mathrm{MHz}$

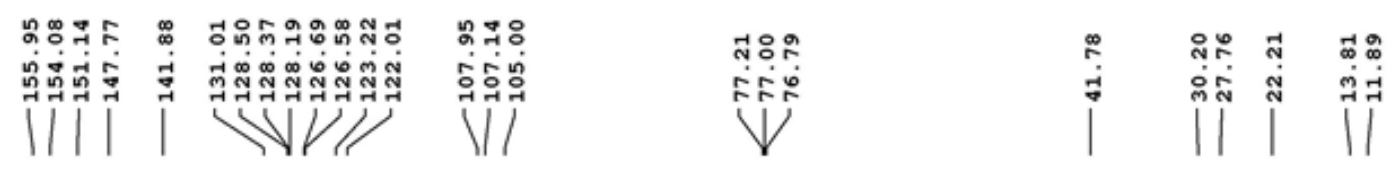

\section{RDK-02-223}

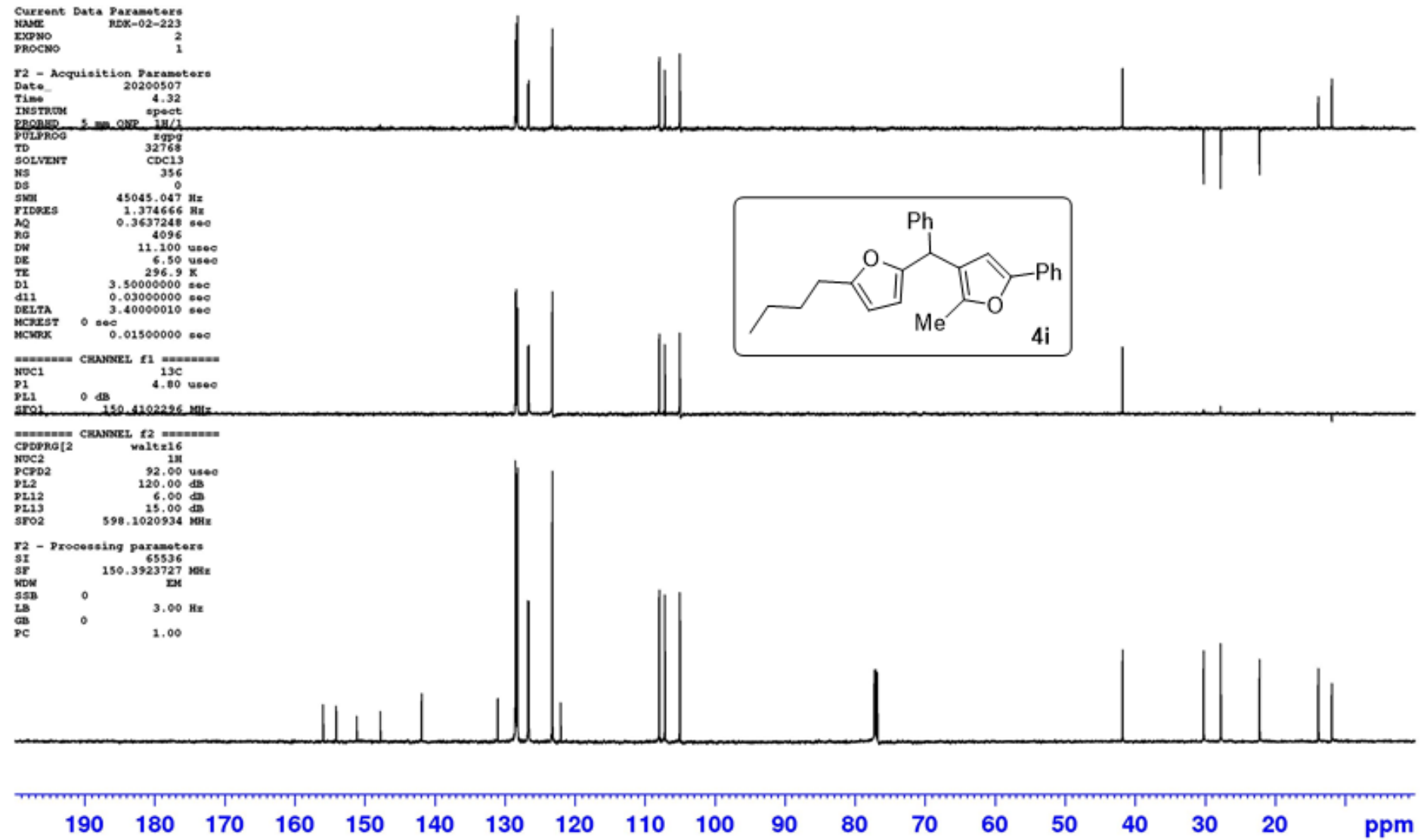


Solvent: $\mathrm{CDCl}_{3}$

SFO1: $600 \mathrm{MHz}$

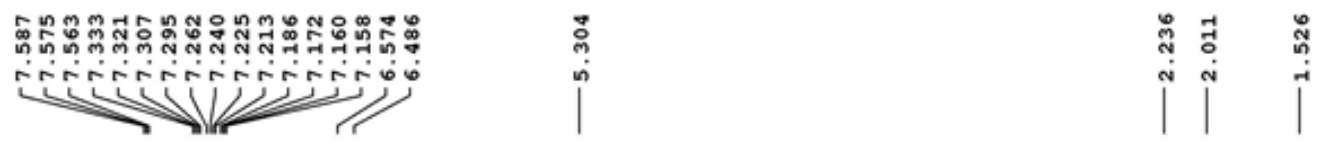

RDK-02-225
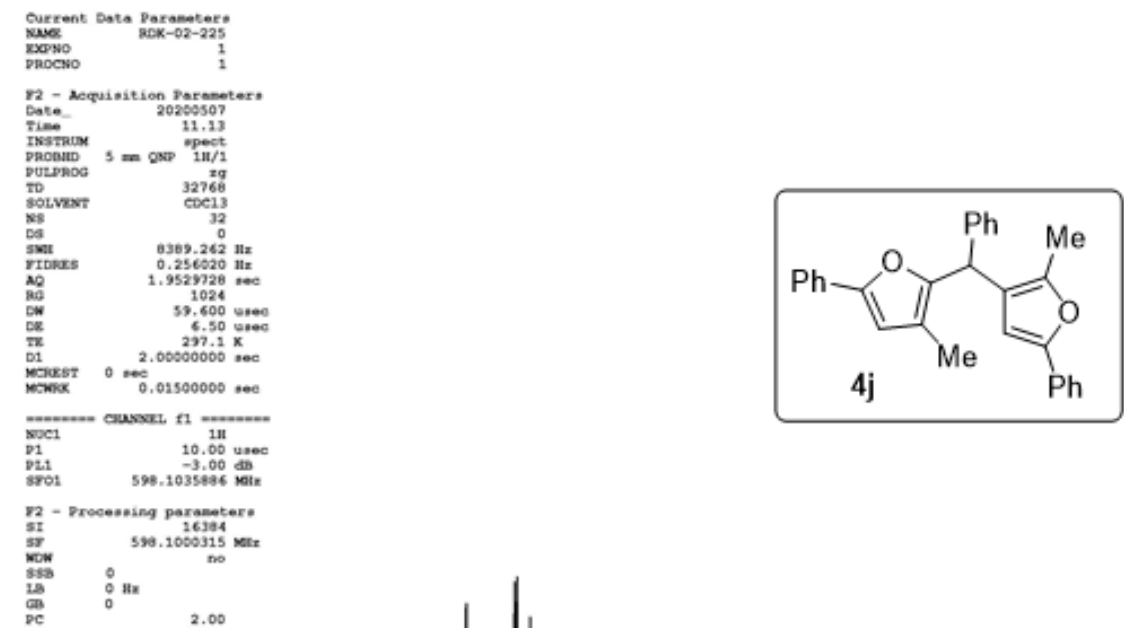

2.00

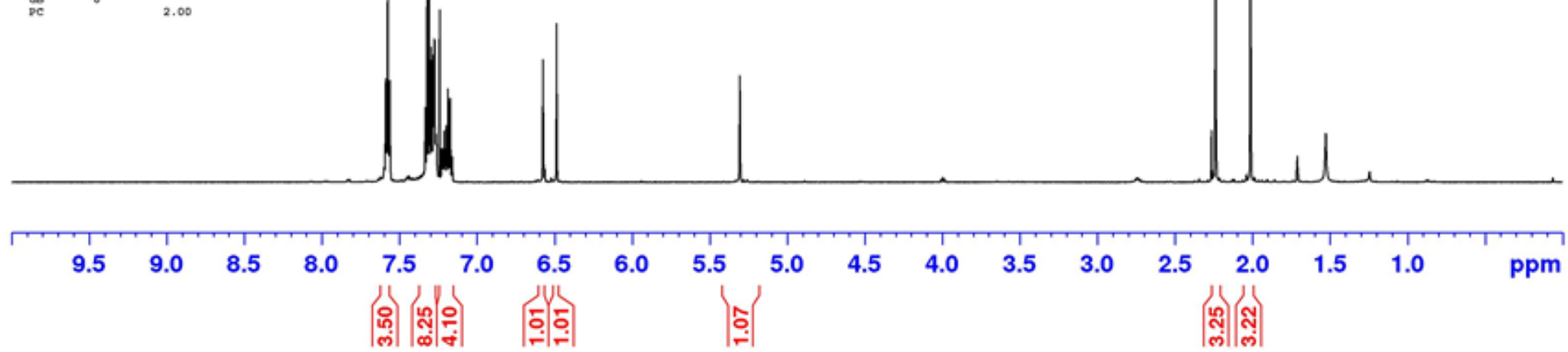


Solvent: $\mathrm{CDCl}_{3}$

SFO1: $150 \mathrm{MHz}$

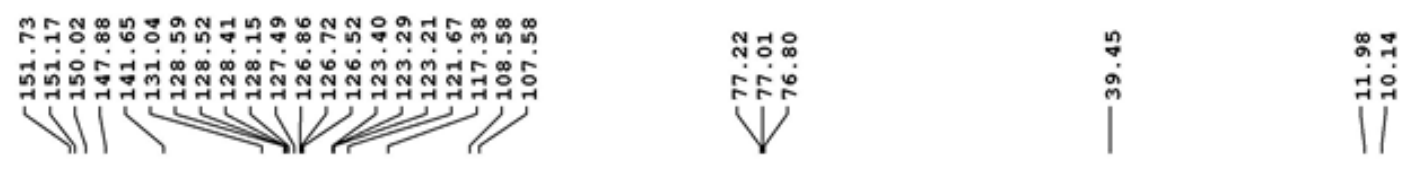

RDK-02-225

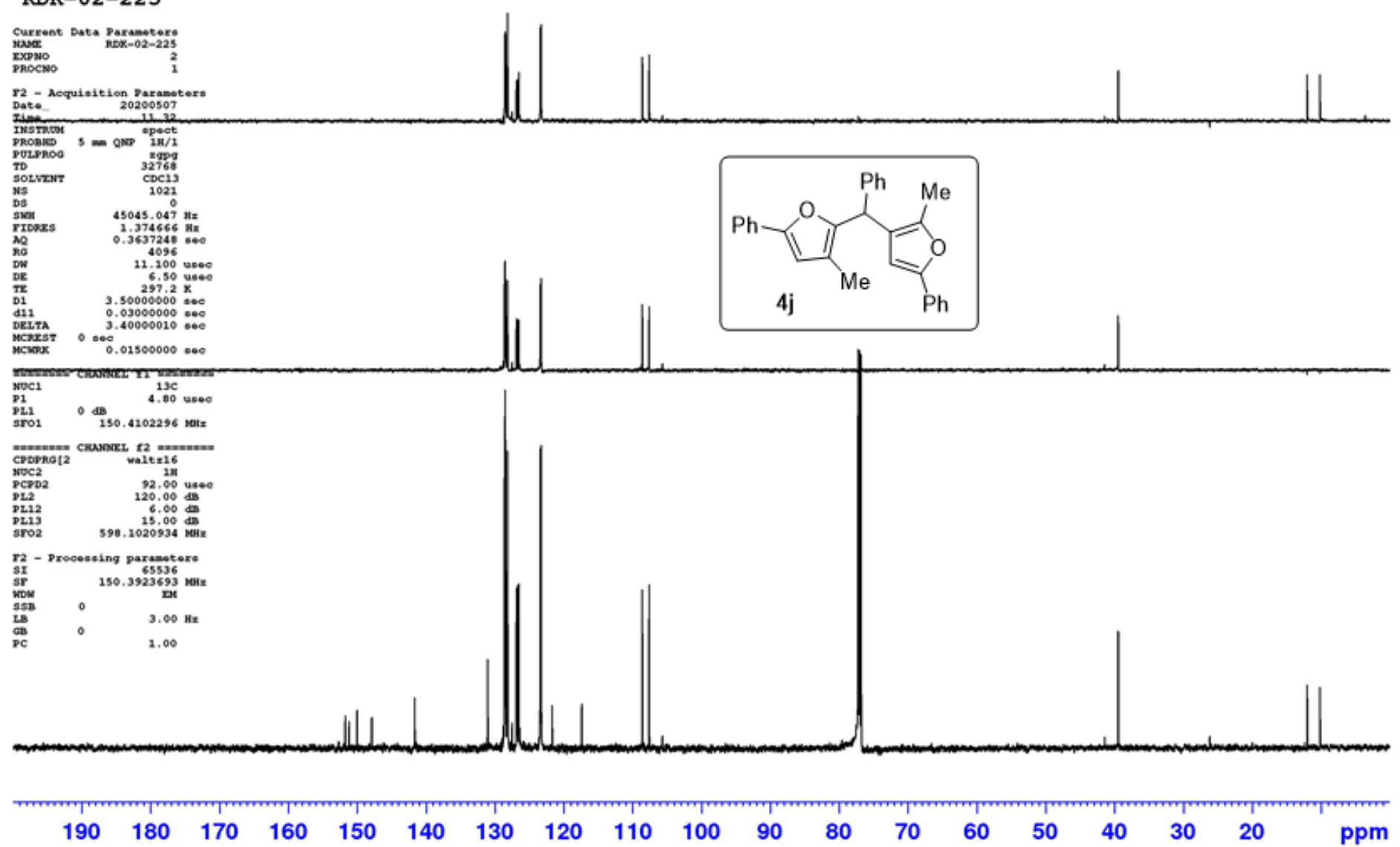


Solvent: $\mathrm{CDCl}_{3}$

SFO1: $600 \mathrm{MHz}$

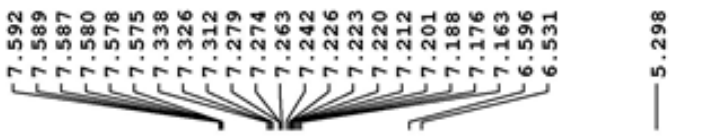

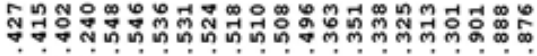

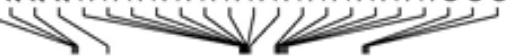

RDK-02-229
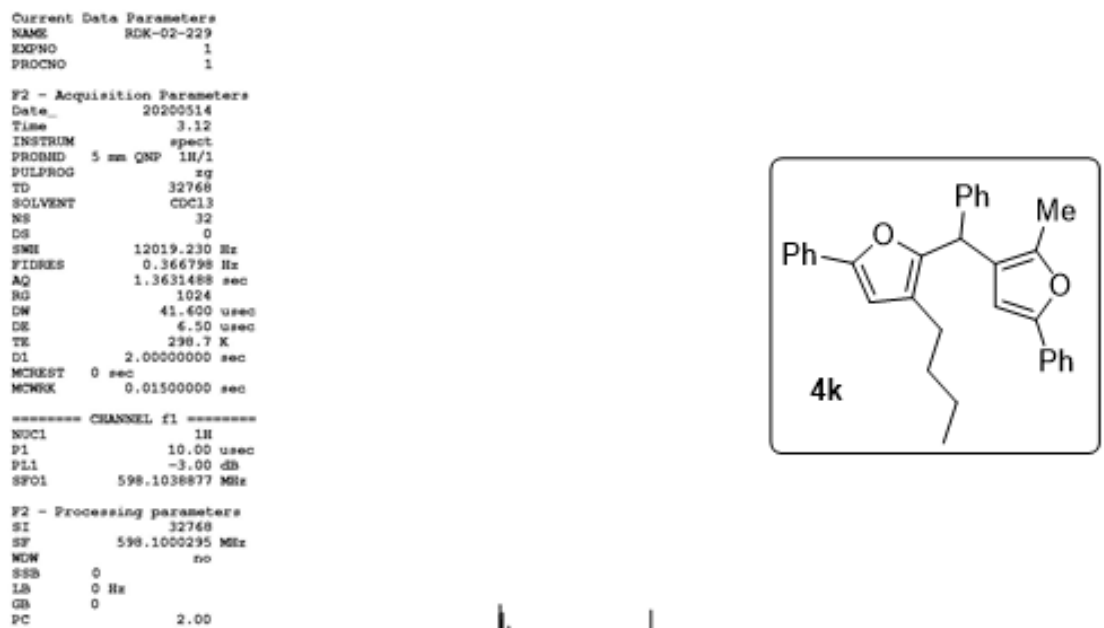

2.00

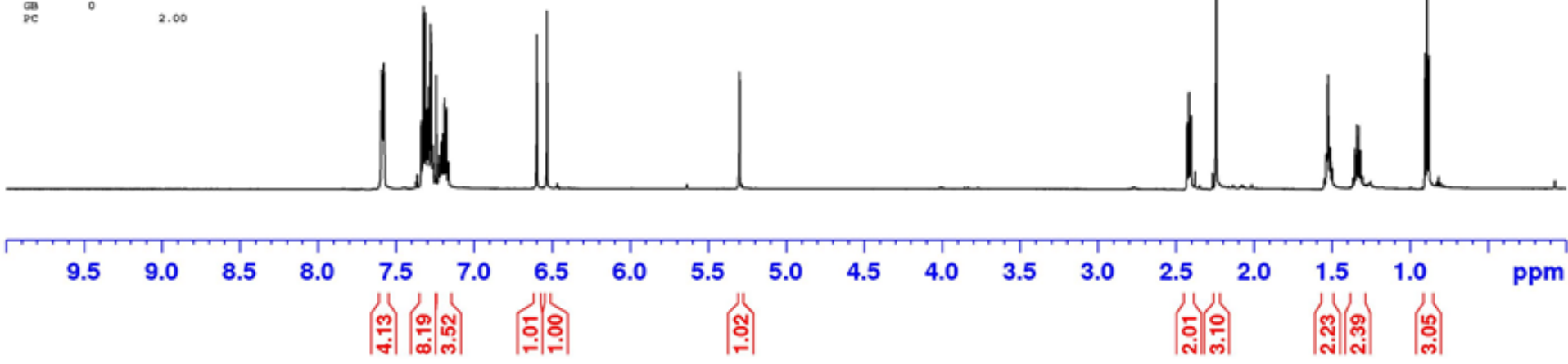


Solvent: $\mathrm{CDCl}_{3}$

SFO1: $150 \mathrm{MHz}$
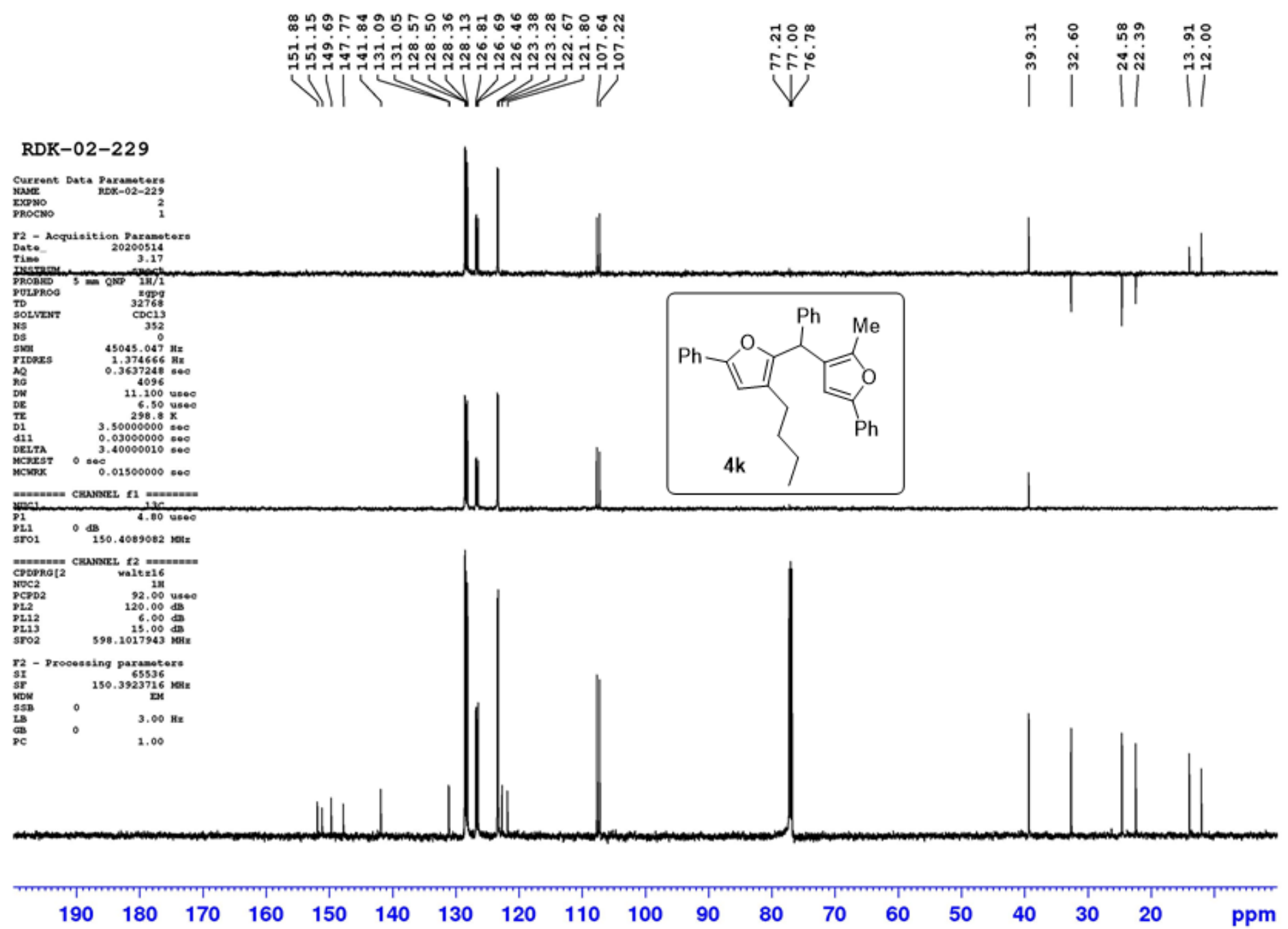
Solvent: $\mathrm{CDCl}_{3}$

SFO1: $600 \mathrm{MHz}$

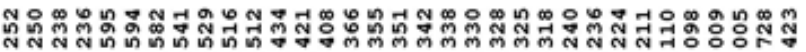

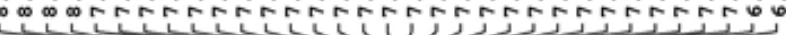

(

RDK-03-08-2A

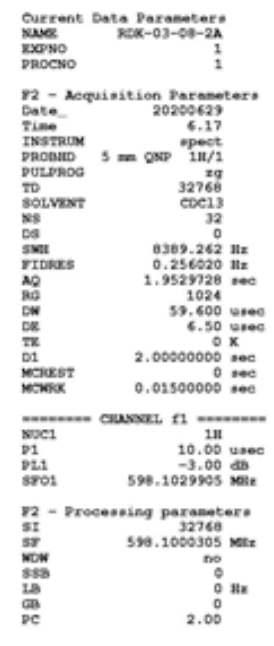
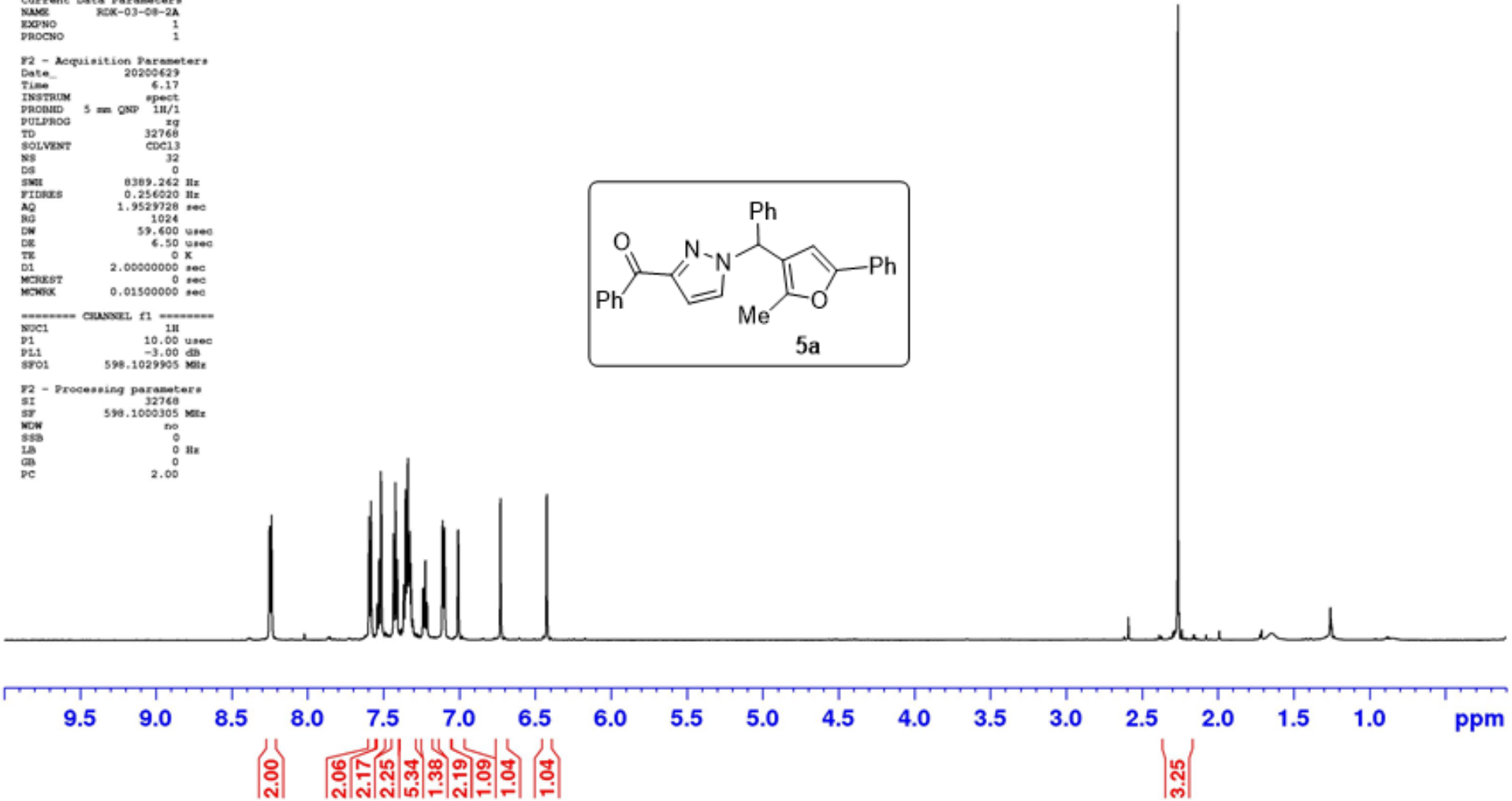
Solvent: $\mathrm{CDCl}_{3}$

SFO1: $150 \mathrm{MHz}$

$$
\text { | }
$$

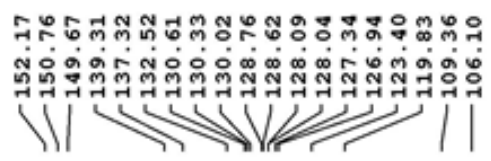

สำㅇำ

ตุ

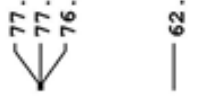

o

సั

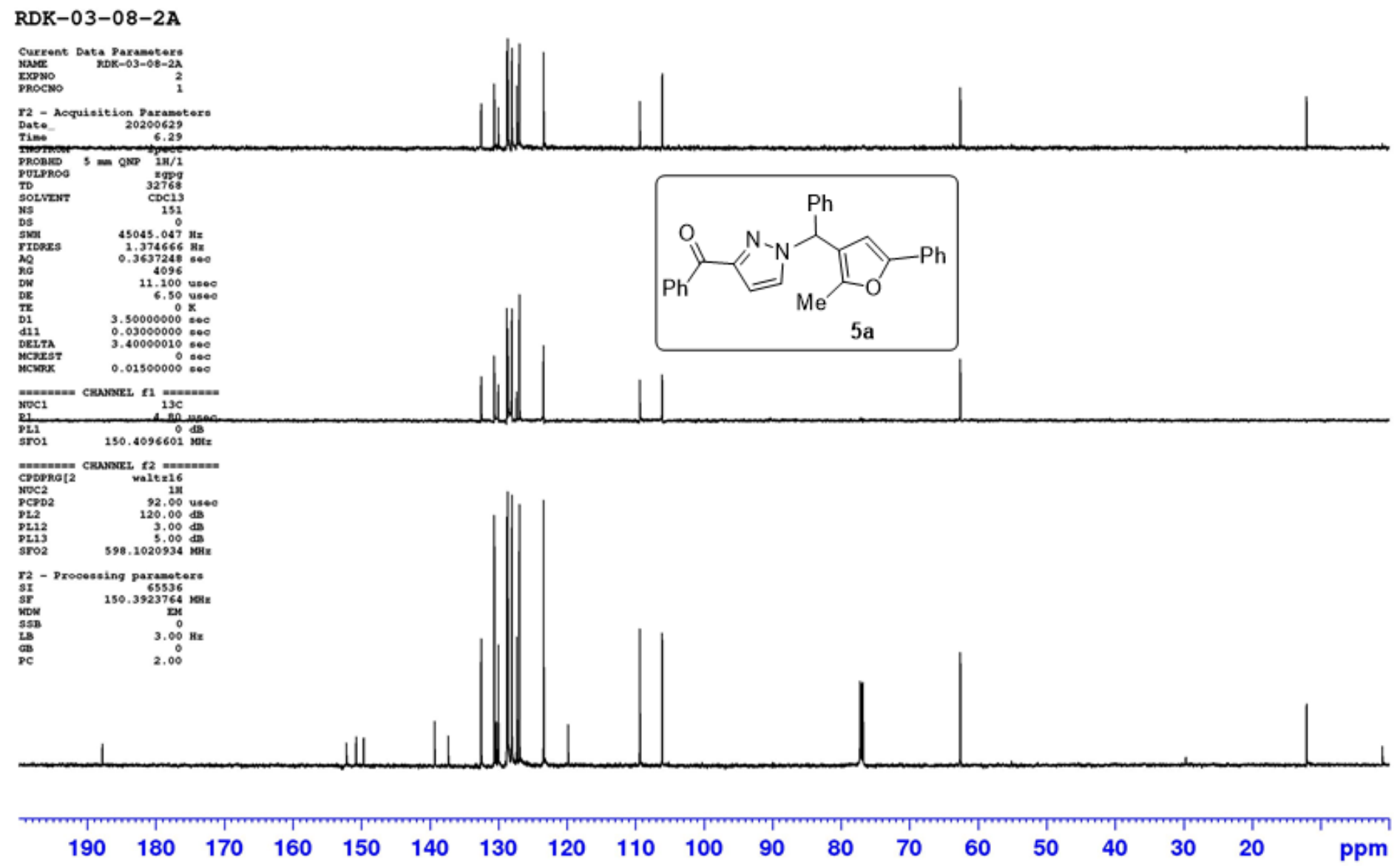


Solvent: $\mathrm{CDCl}_{3}$

SFO1: $600 \mathrm{MHz}$

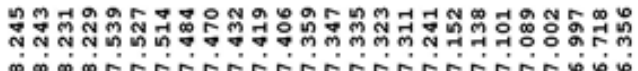

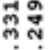

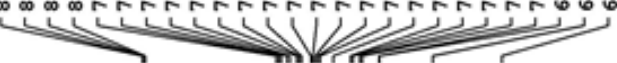

RDK-03-14
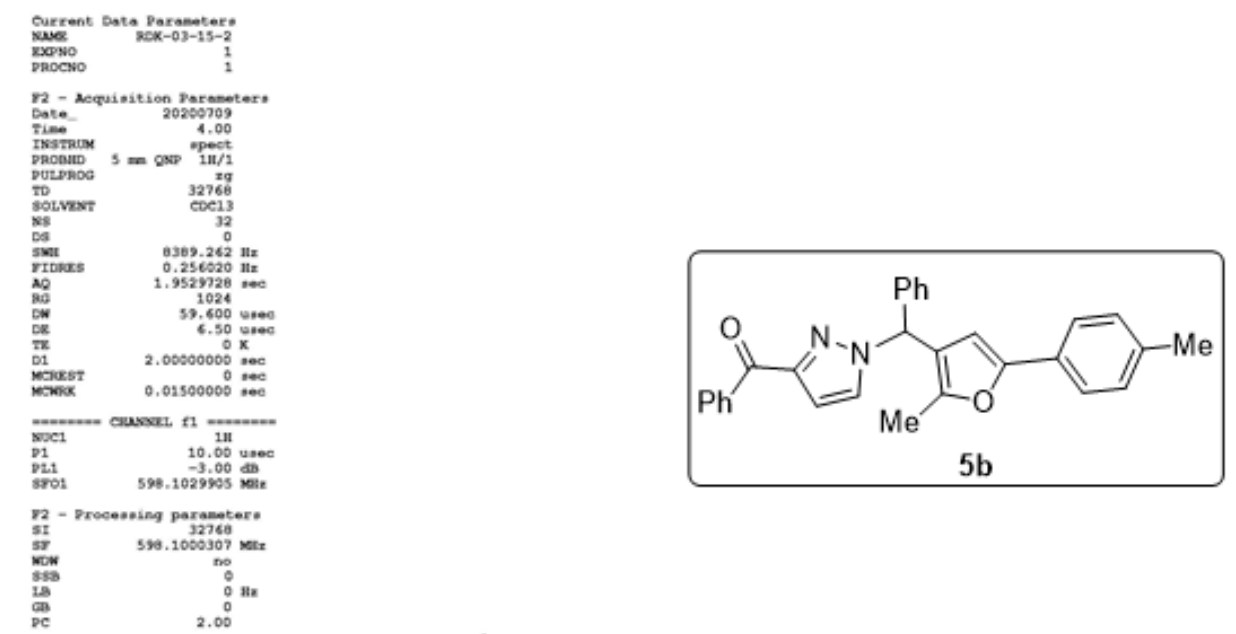

$$
\text { PC } 2.00
$$

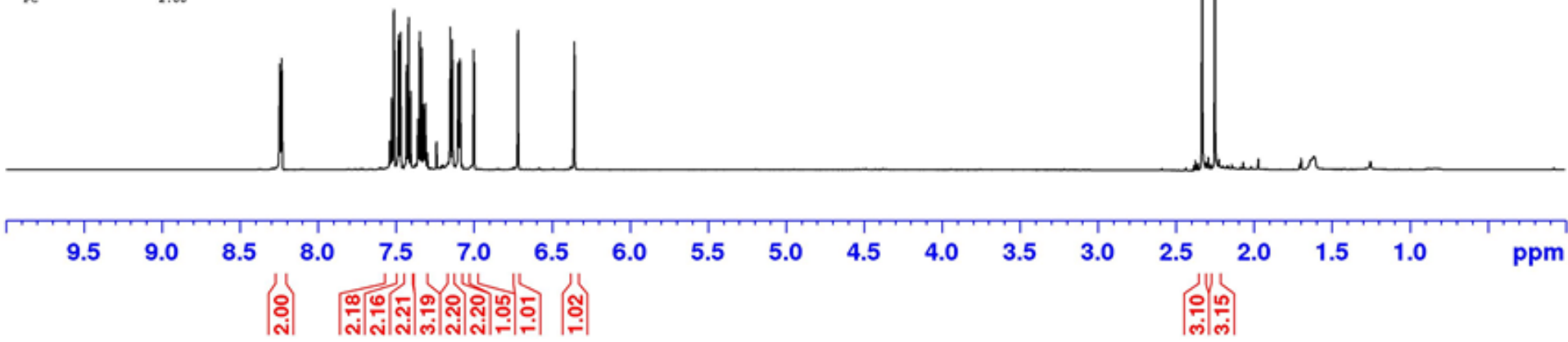


Solvent: $\mathrm{CDCl}_{3}$ SFO1: $150 \mathrm{MHz}$
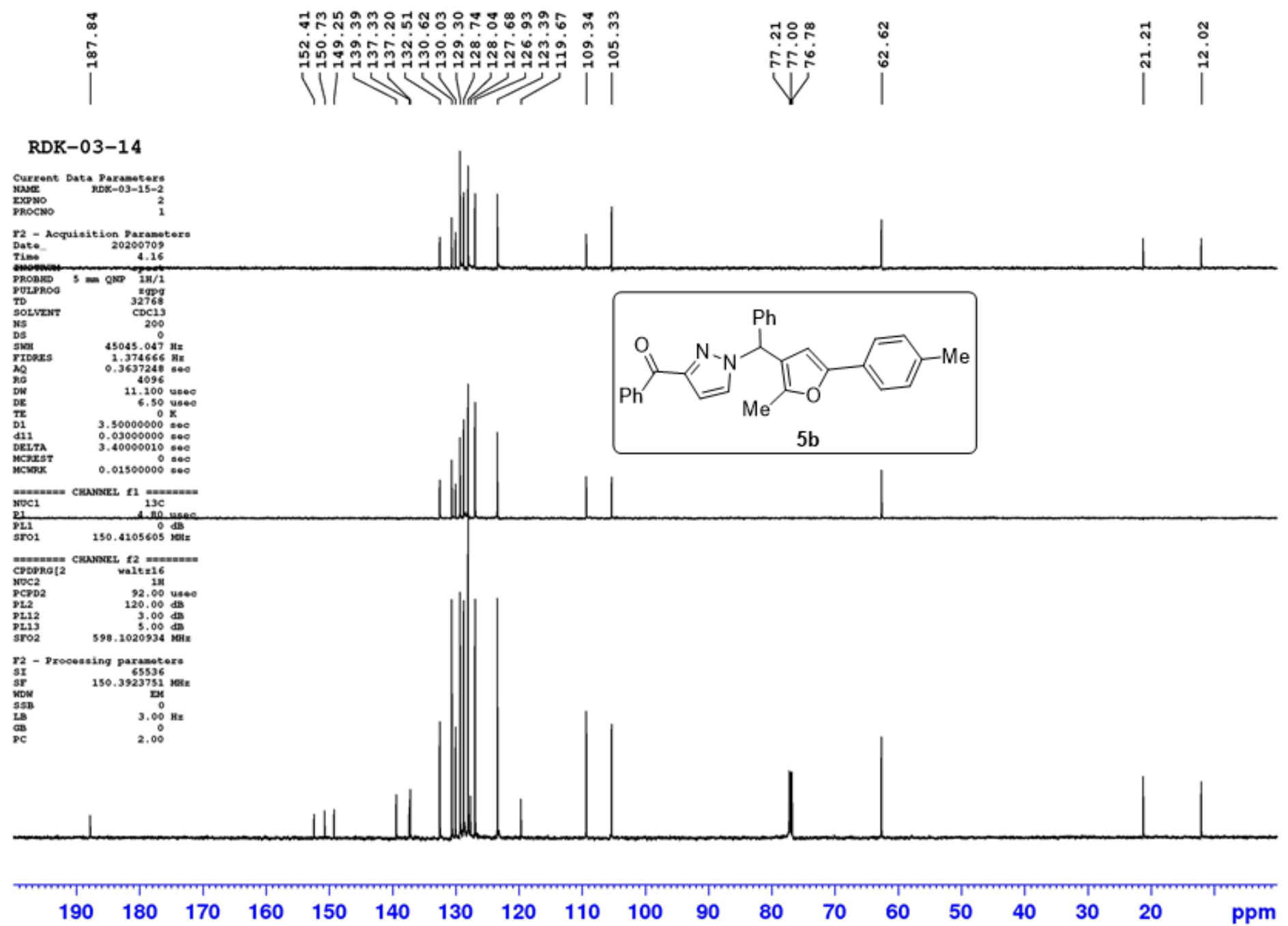
Solvent: $\mathrm{CDCl}_{3}$

SFO1: $600 \mathrm{MHz}$

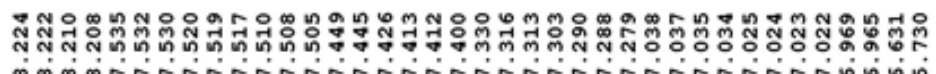

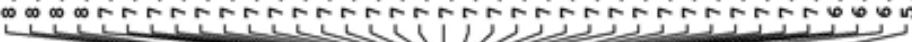

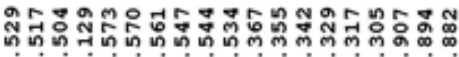

N

RDK-03-15

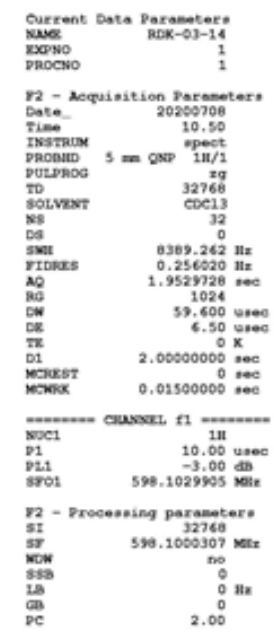
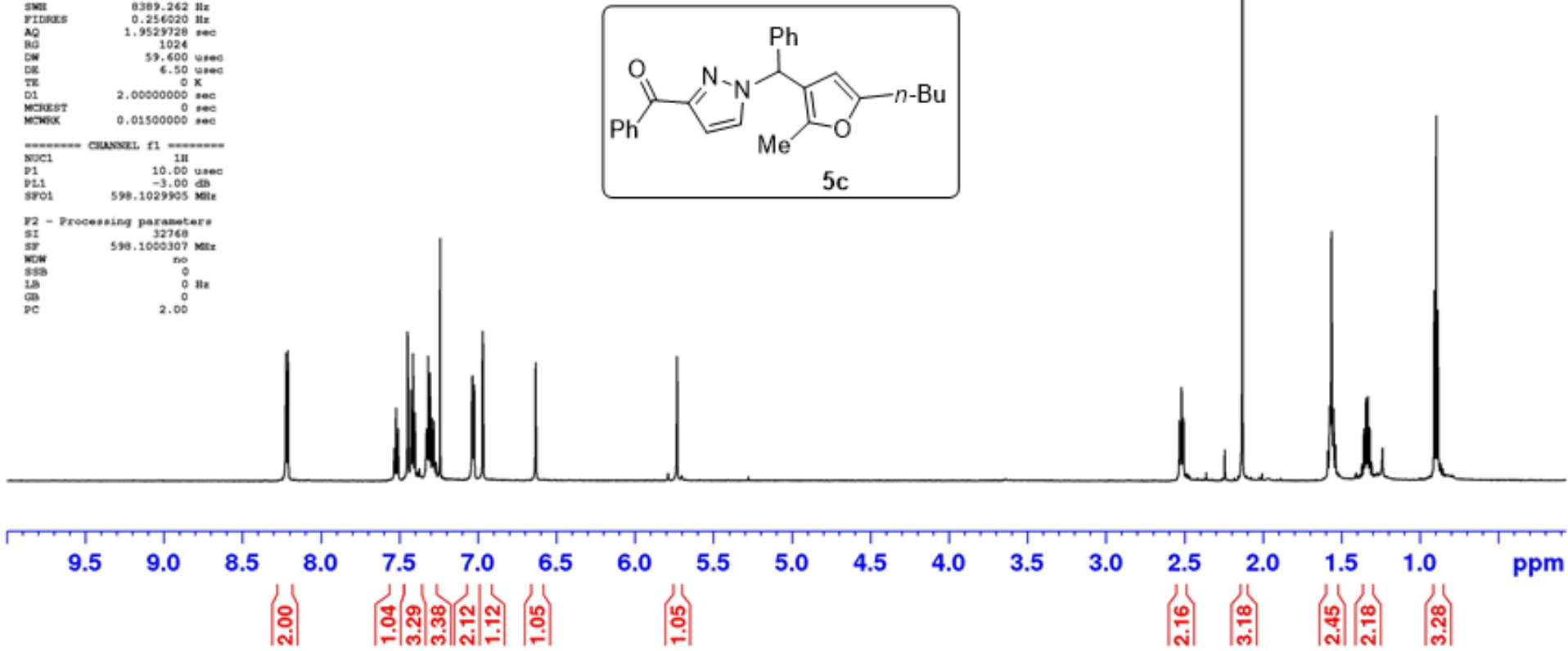
Solvent: $\mathrm{CDCl}_{3}$ SFO1: $150 \mathrm{MHz}$

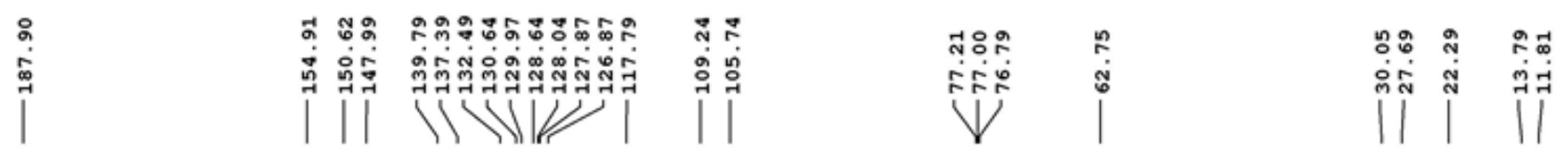

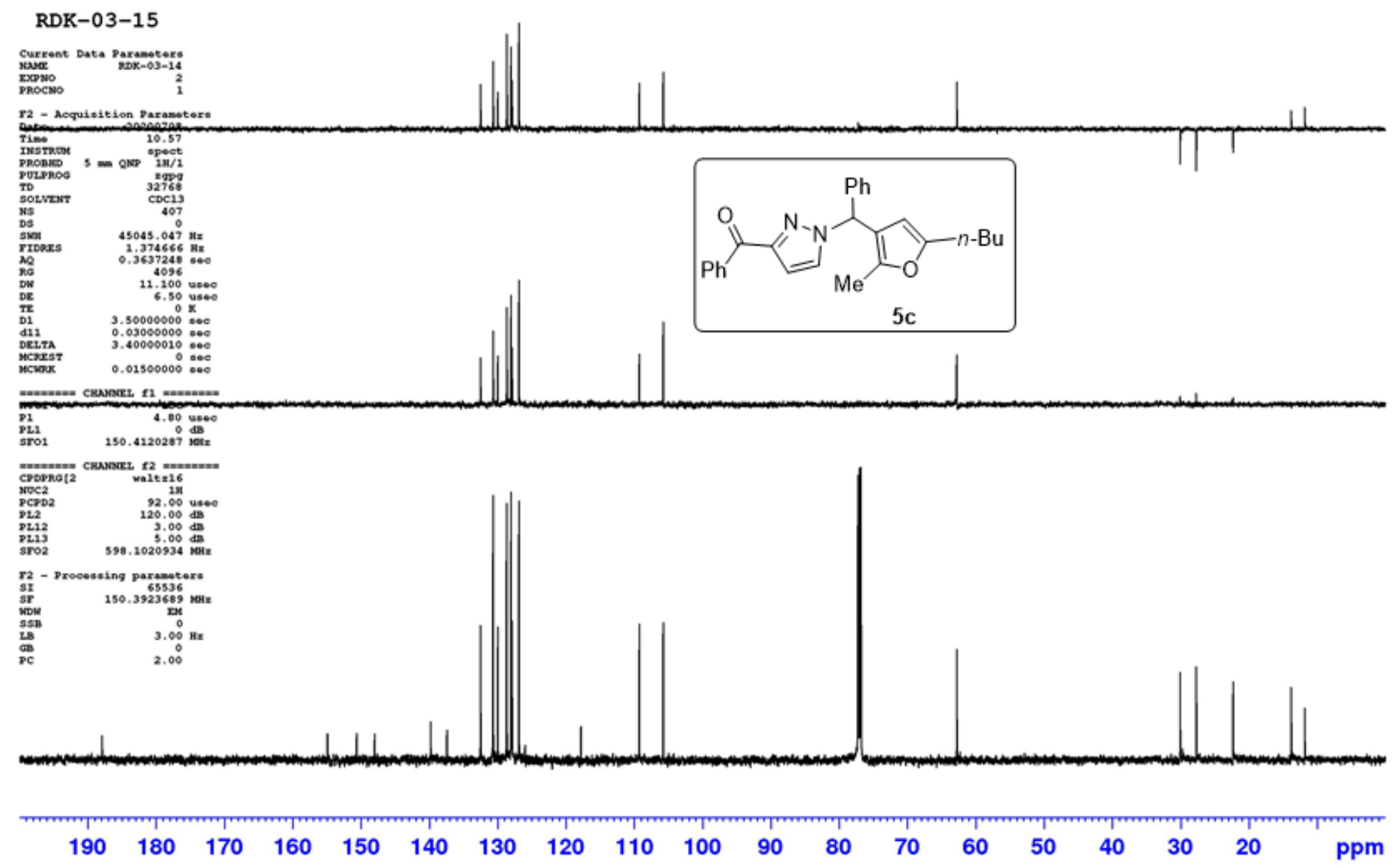


Solvent: $\mathrm{CDCl}_{3}$

SFO1: $600 \mathrm{MHz}$

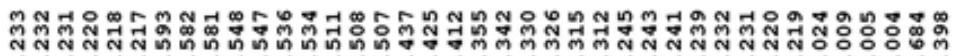

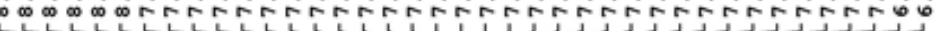

RDK-03-18
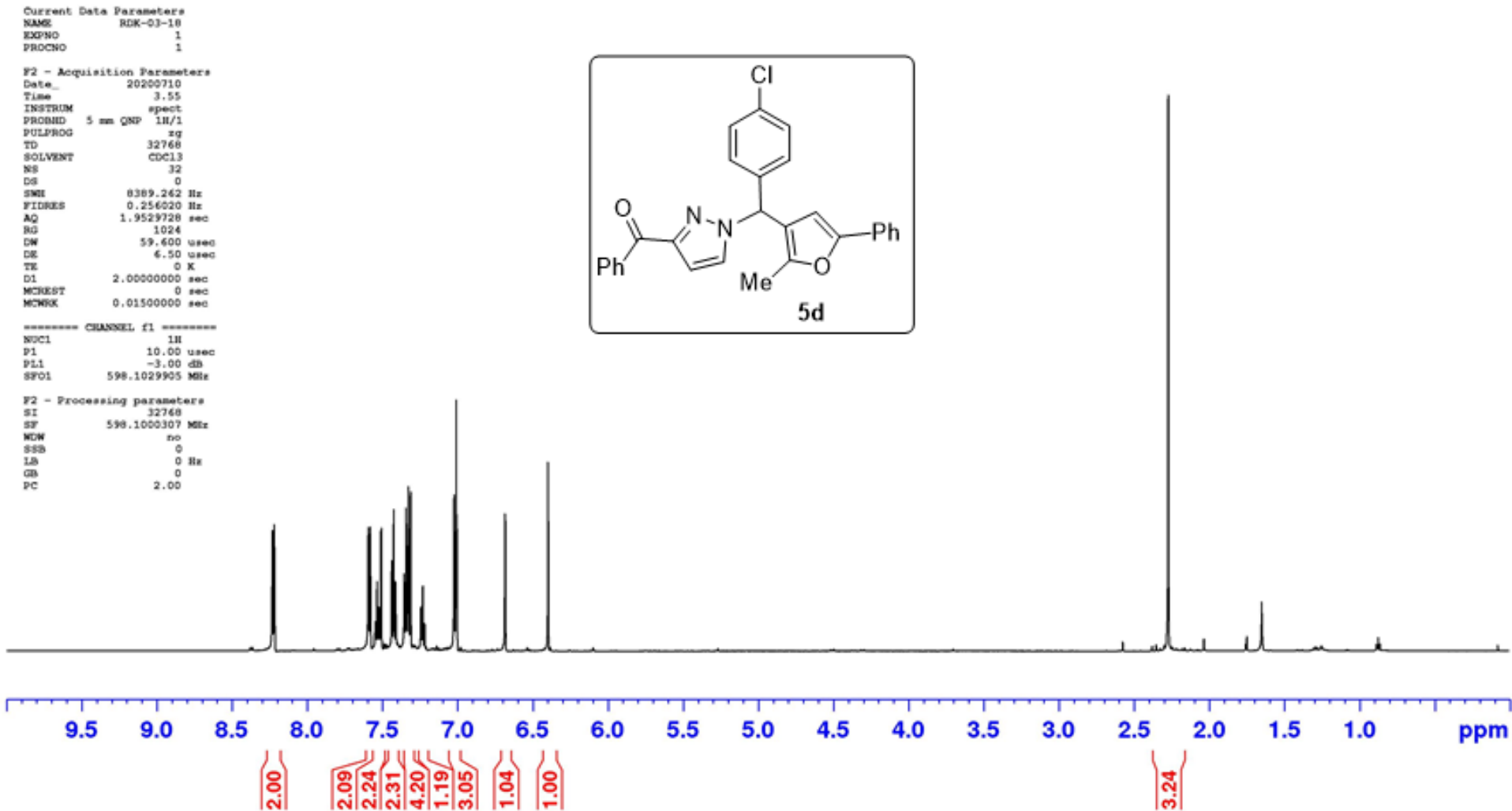
Solvent: $\mathrm{CDCl}_{3}$ SFO1: $150 \mathrm{MHz}$

$$
\stackrel{\circ}{\stackrel{0}{0}}
$$

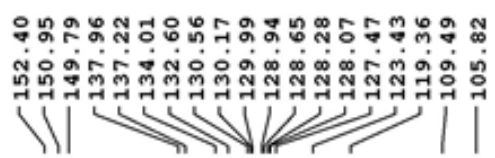

고응

ง

ㄷํ

$\div$

구

RDK-03-18

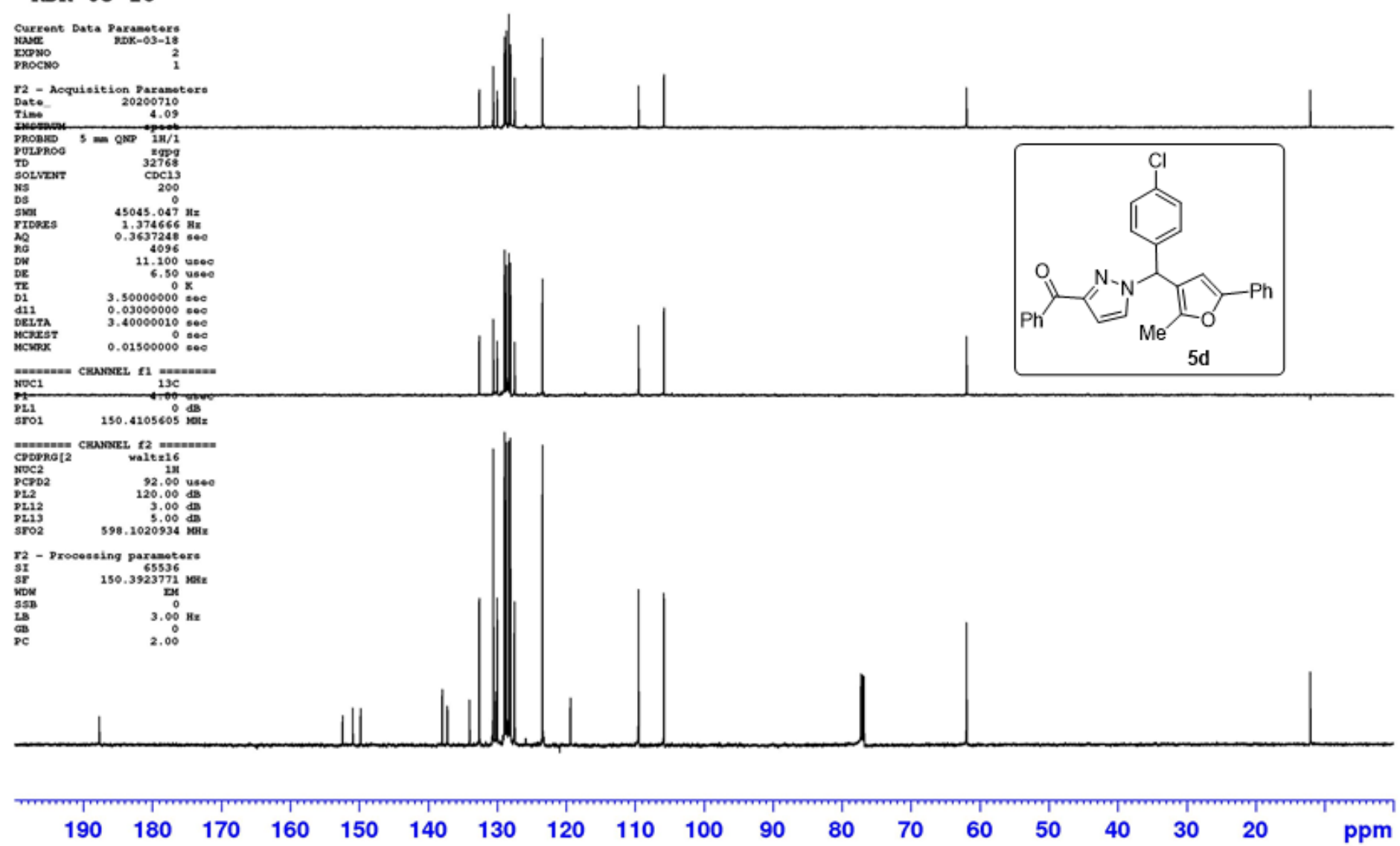


Solvent: $\mathrm{CDCl}_{3}$

SFO1: $700 \mathrm{MHz}$
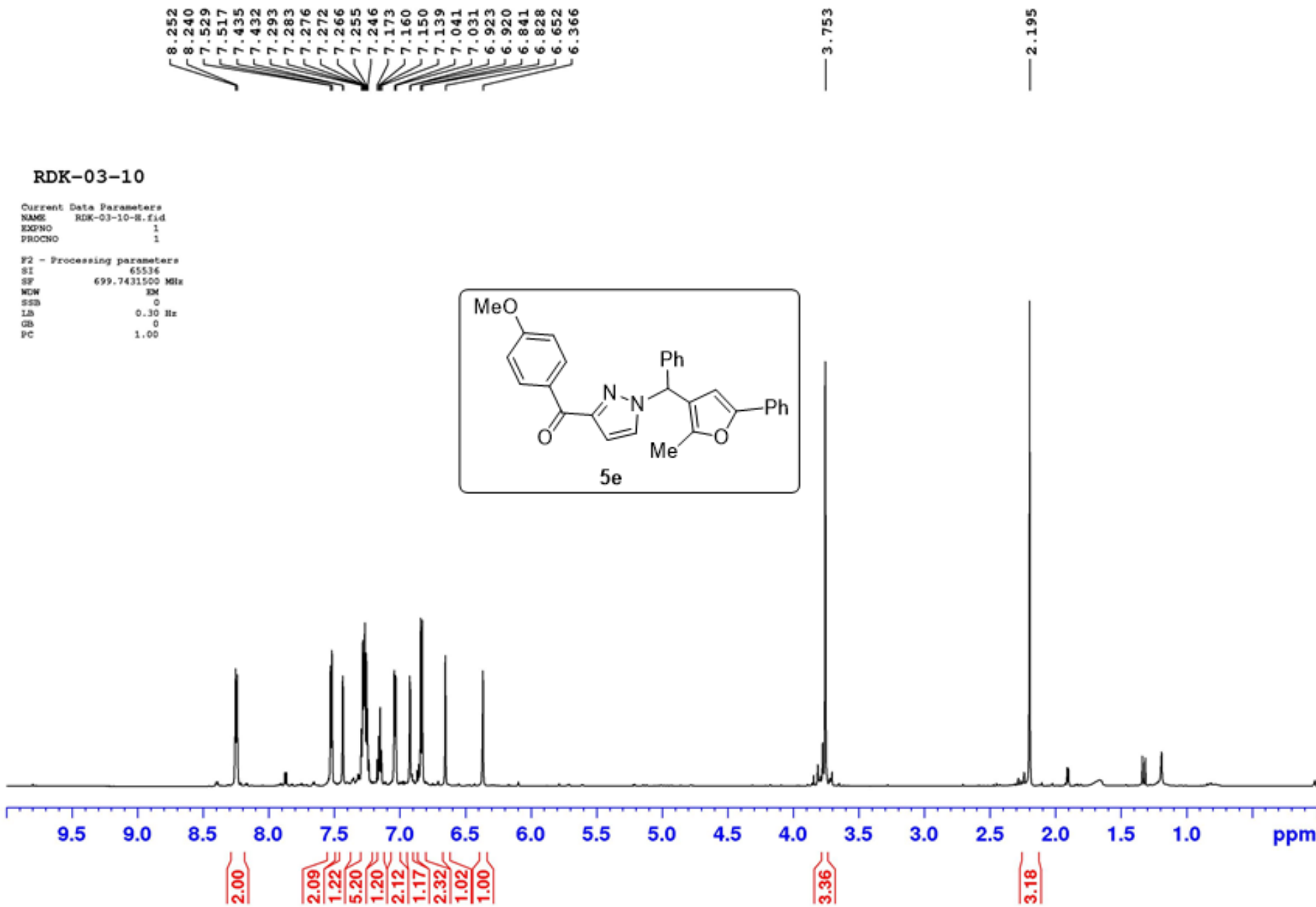
Solvent: $\mathrm{CDCl}_{3}$

SFO1: $175 \mathrm{MHz}$
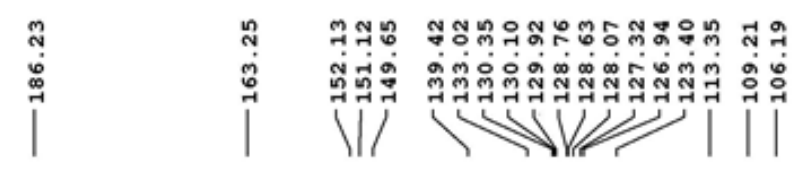

뜸ำ

당

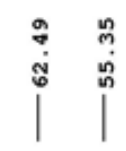

옹

ㄱำ

RDK-03-10
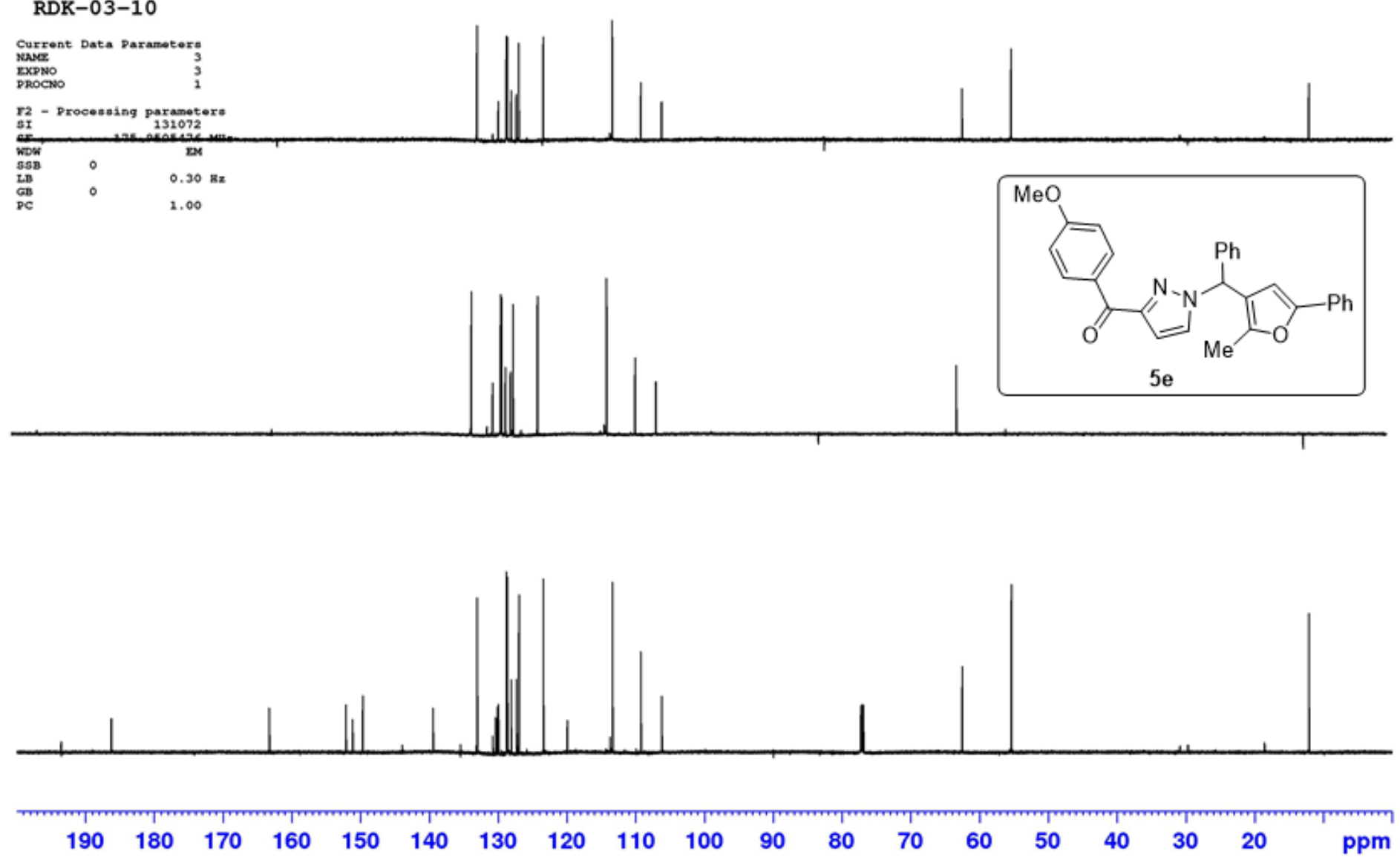
Solvent: $\mathrm{CDCl}_{3}$

SFO1: $700 \mathrm{MHz}$

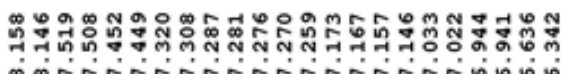

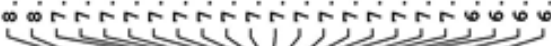

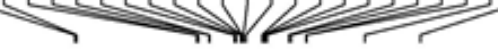
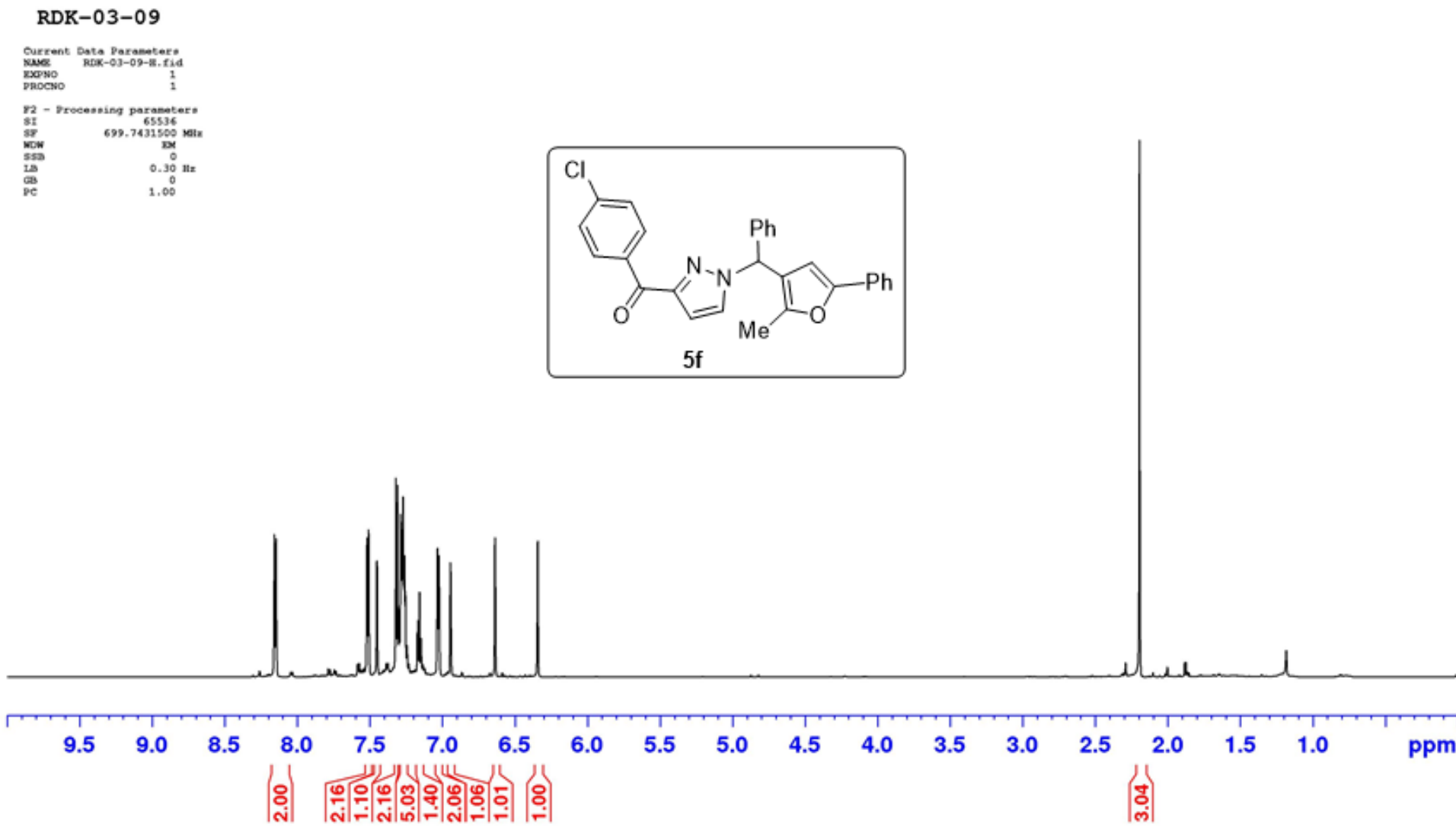
Solvent: $\mathrm{CDCl}_{3}$

SFO1: $175 \mathrm{MHz}$
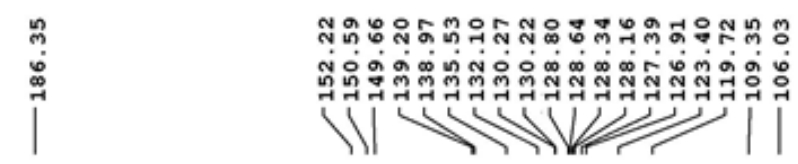

๓

6

ㄱำ

RDK-03-09
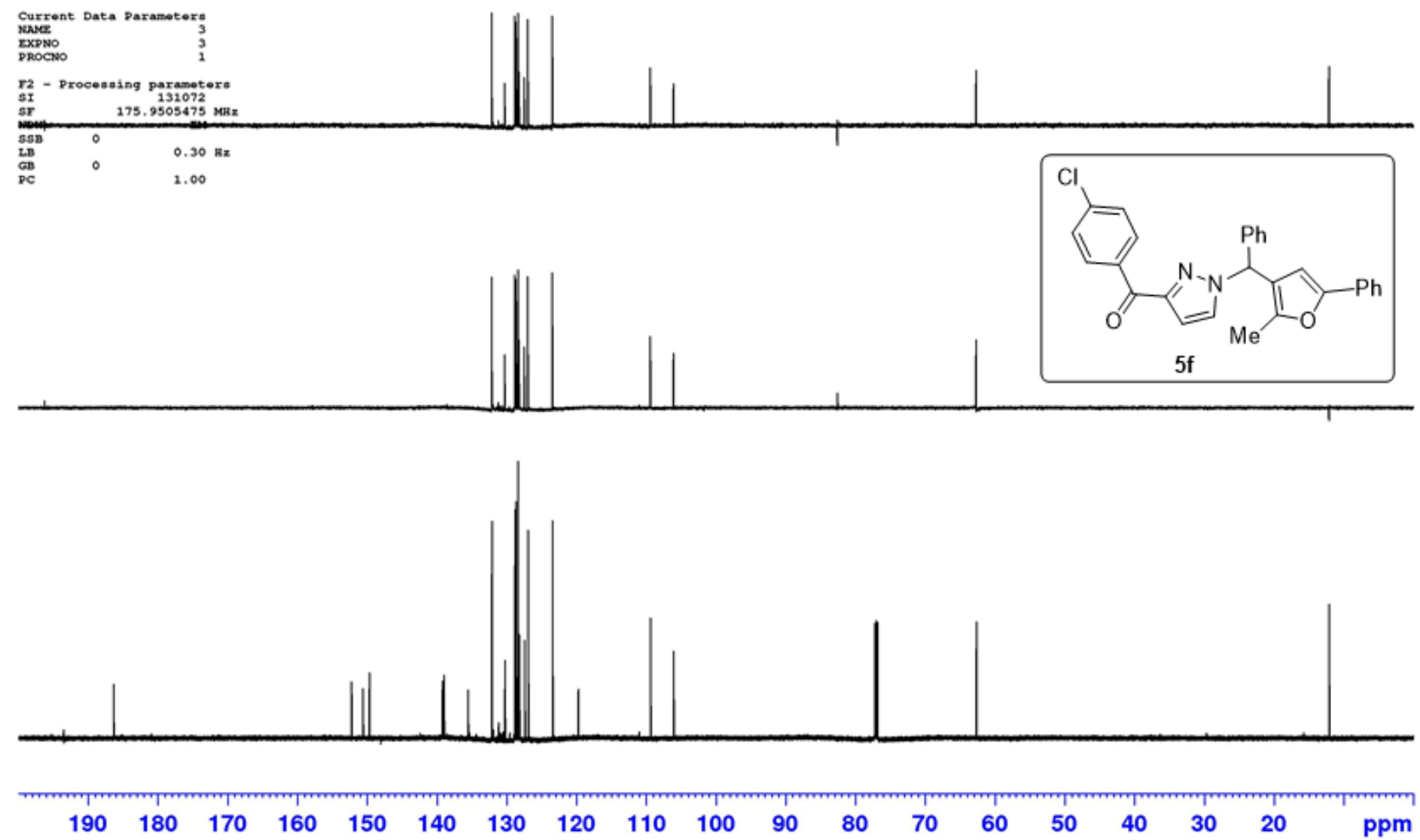
Solvent: $\mathrm{CDCl}_{3}$

SFO1: $700 \mathrm{MHz}$

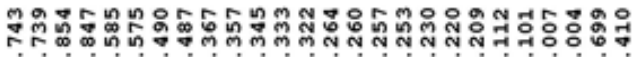

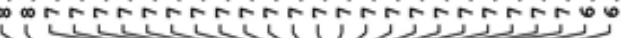

ஸั

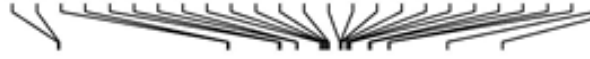

RDK-03-11

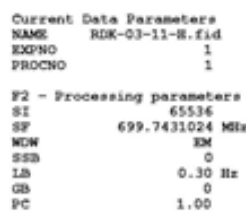
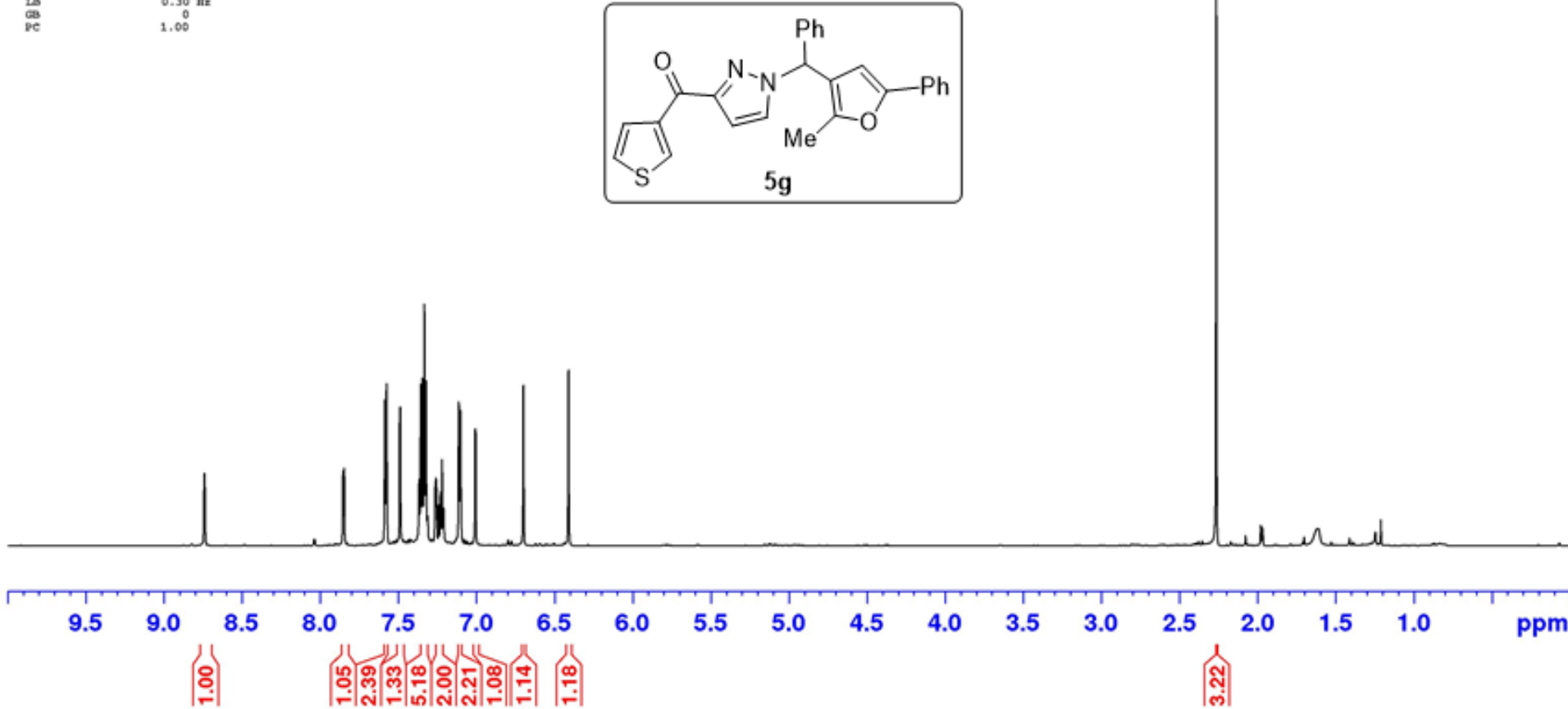
Solvent: $\mathrm{CDCl}_{3}$

SFO1: $175 \mathrm{MHz}$
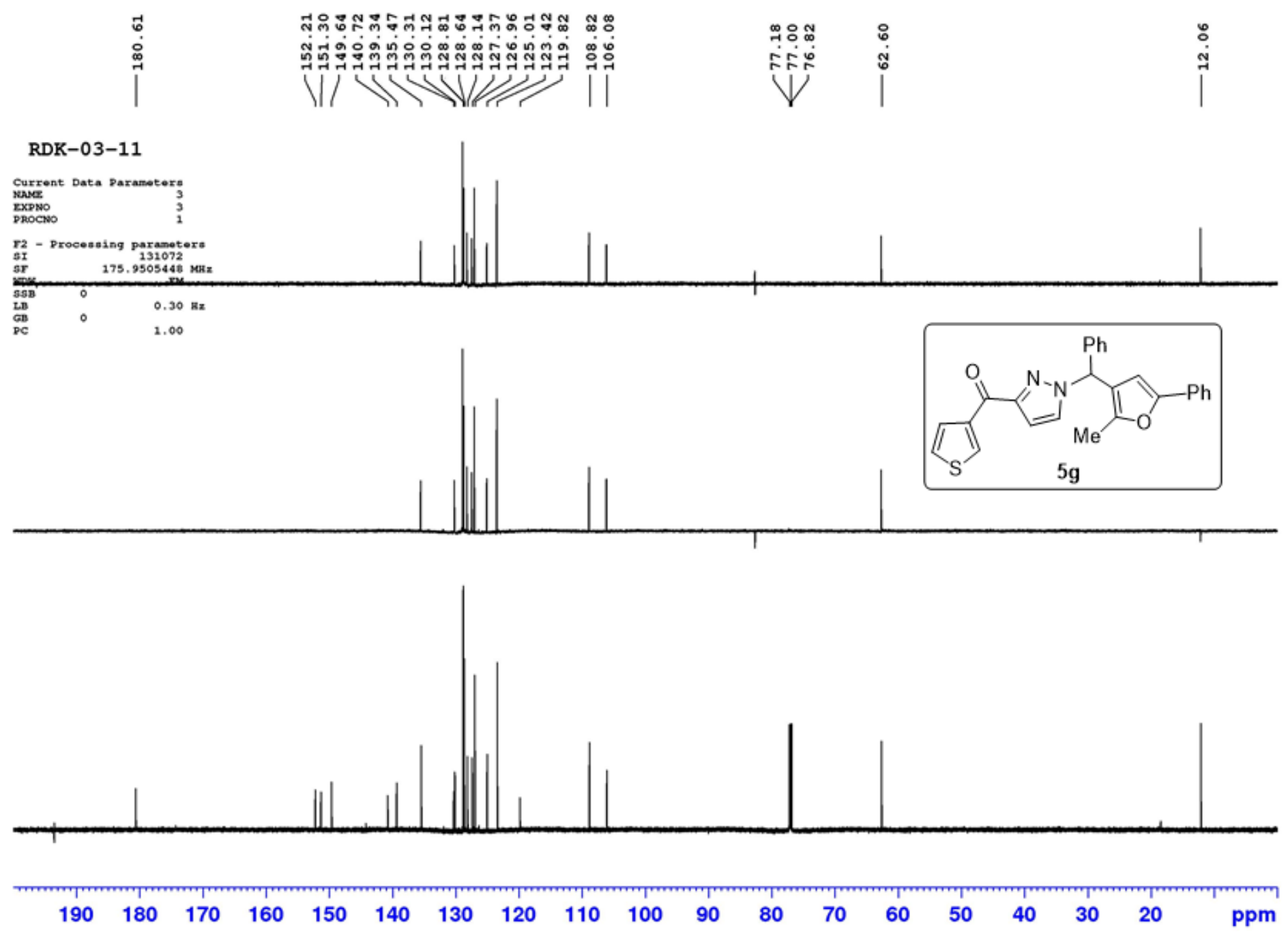
Solvent: $\mathrm{CDCl}_{3}$

SFO1: $700 \mathrm{MHz}$

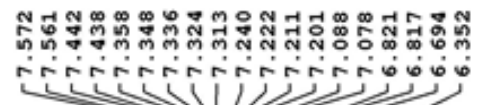

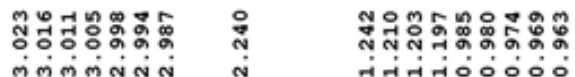

نูvinivi i

\section{RDK-03-12}

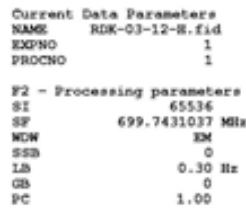
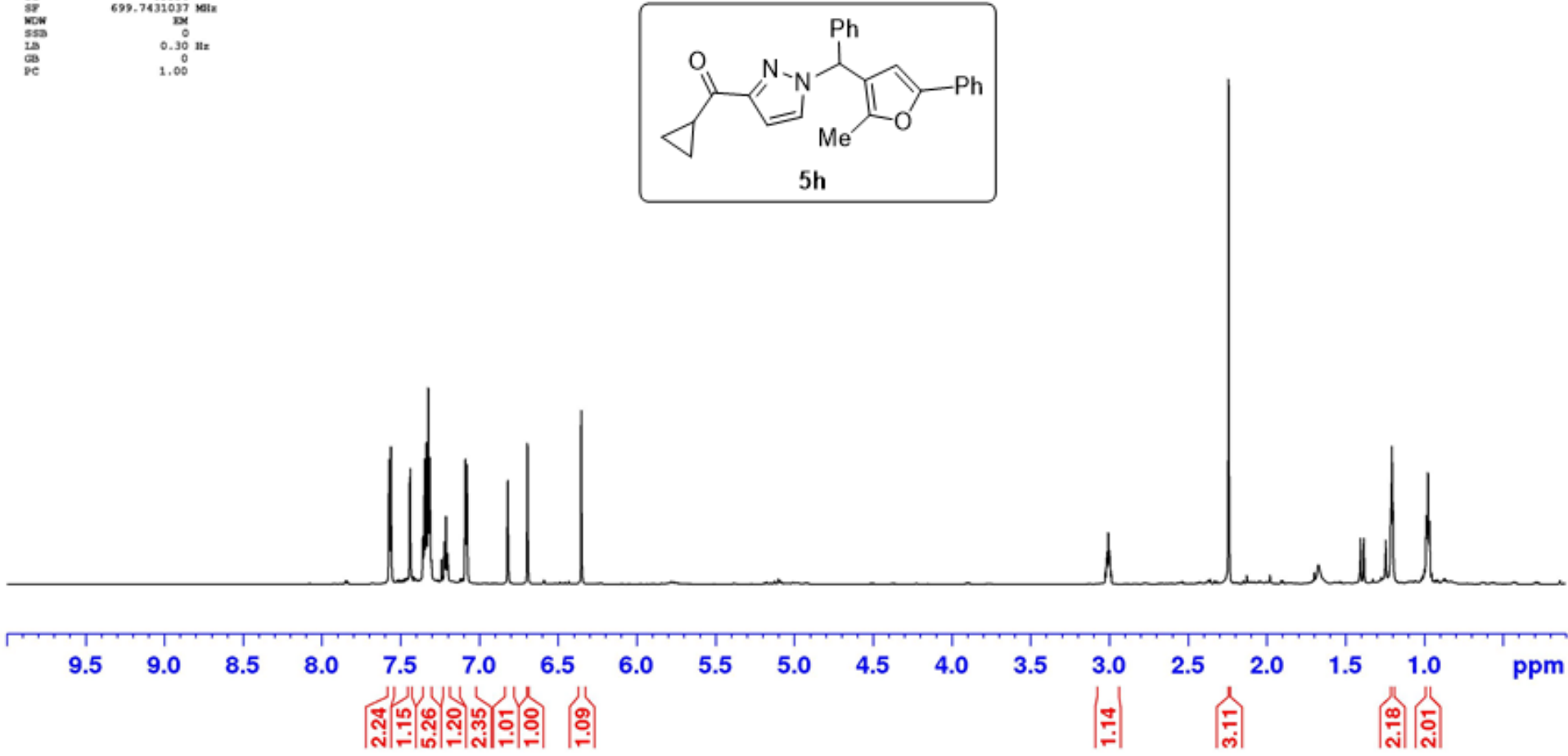
Solvent: $\mathrm{CDCl}_{3}$ SFO1: $175 \mathrm{MHz}$
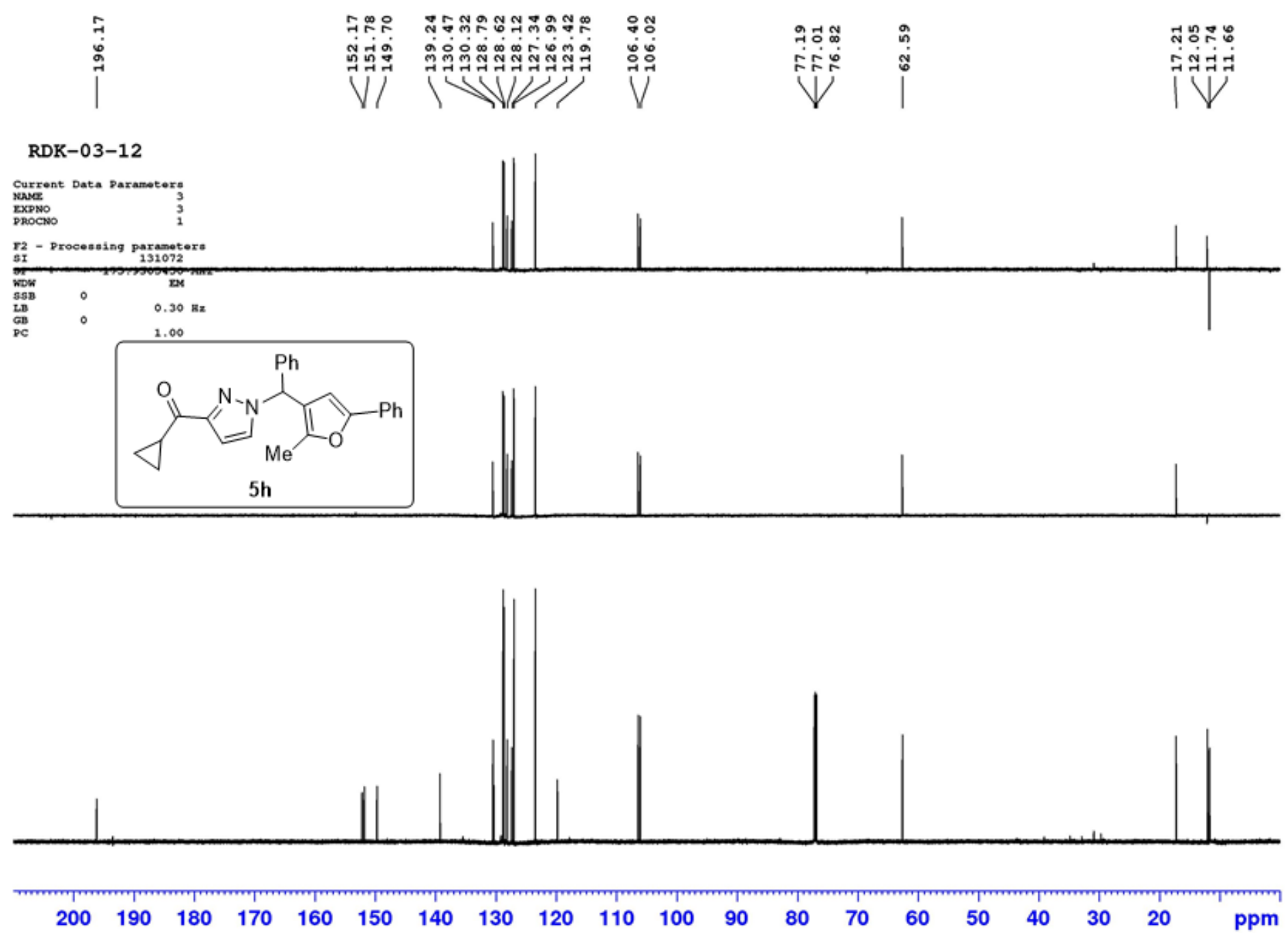
Solvent: $\mathrm{CDCl}_{3}$

SFO1: $700 \mathrm{MHz}$

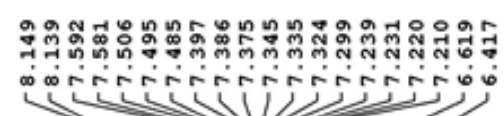

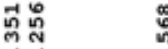

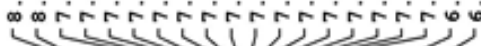

in

RDK-03-13

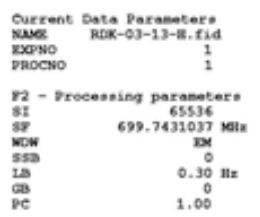
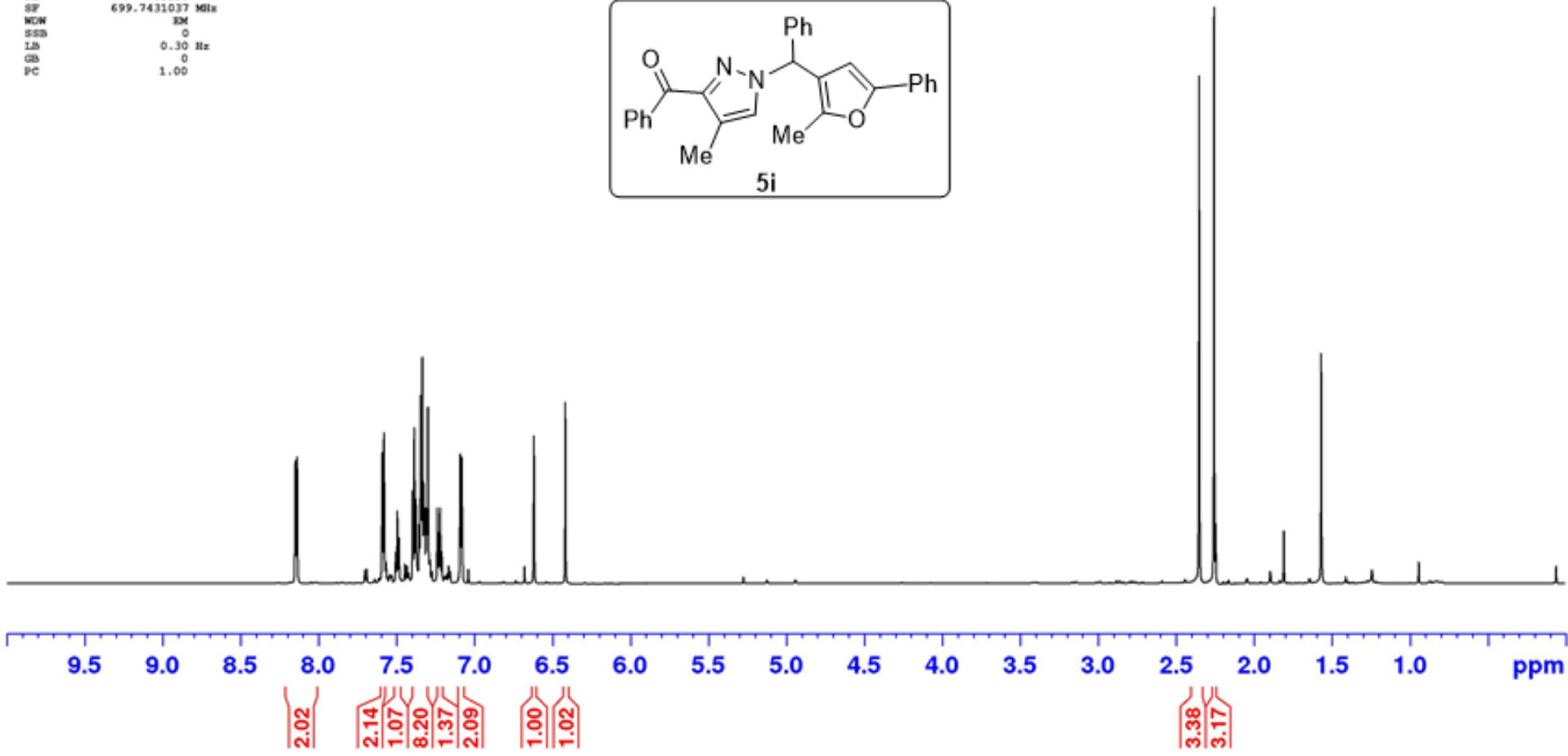
Solvent: $\mathrm{CDCl}_{3}$ SFO1: $175 \mathrm{MHz}$
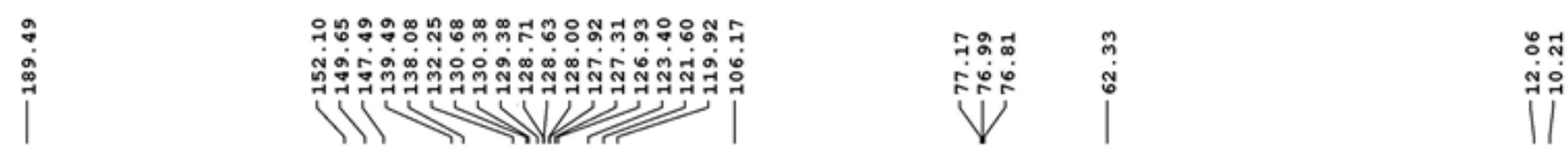

RDK-03-13

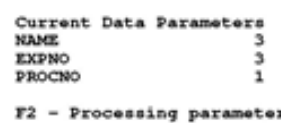

T2 - Processing parametere

sT 131072

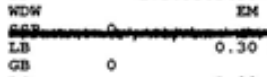

1.00
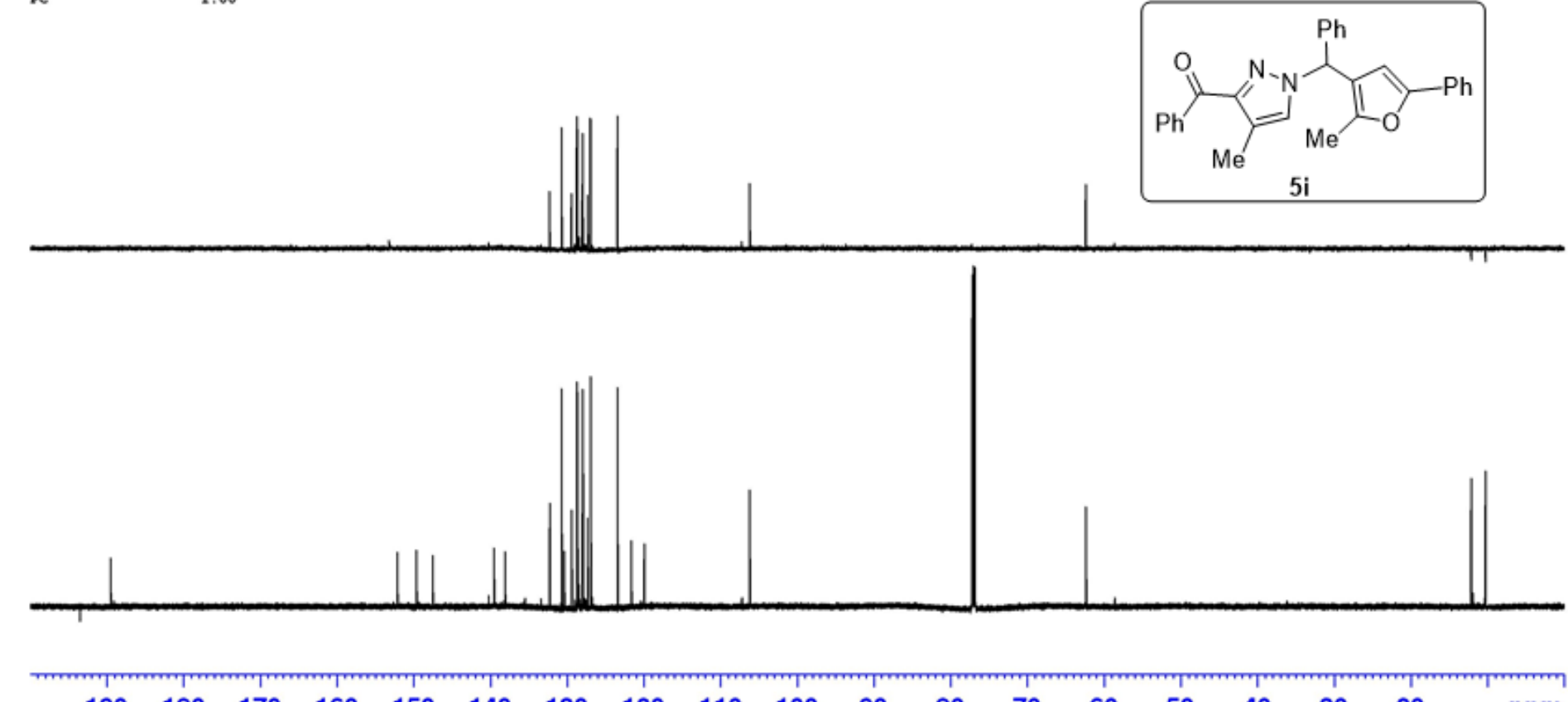

$\begin{array}{lllllllllllllllllll}190 & 180 & 170 & 160 & 150 & 140 & 130 & 120 & 110 & 100 & 90 & 80 & 70 & 60 & 50 & 40 & 30 & 20 & \mathrm{ppm}\end{array}$ 
Solvent: $\mathrm{CDCl}_{3}$

SFO1: $400 \mathrm{MHz}$

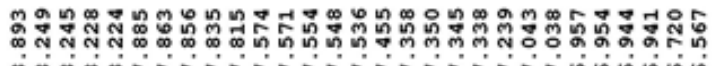

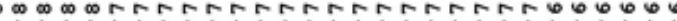

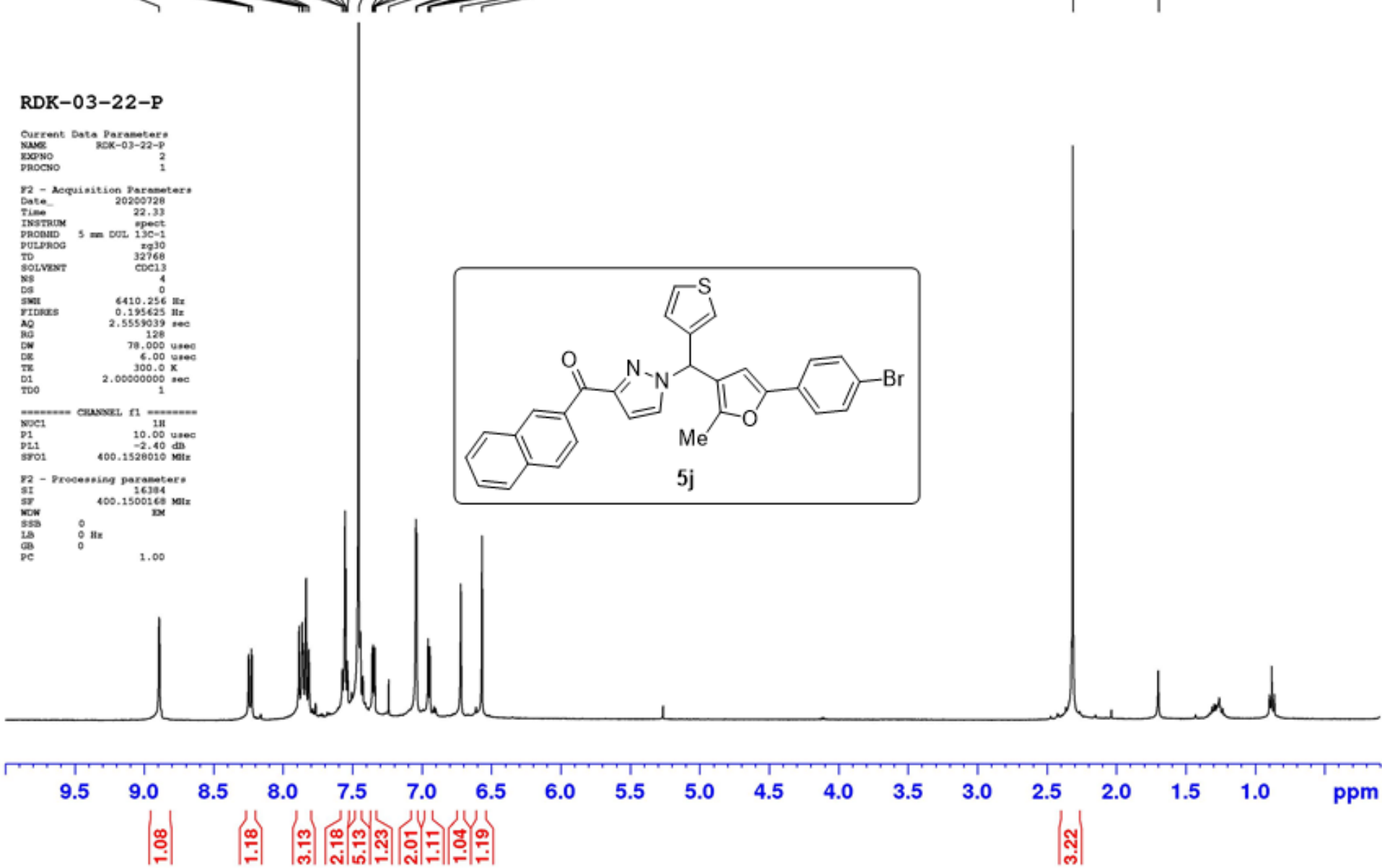


Solvent: $\mathrm{CDCl}_{3}$

SFO1: $100 \mathrm{MHz}$

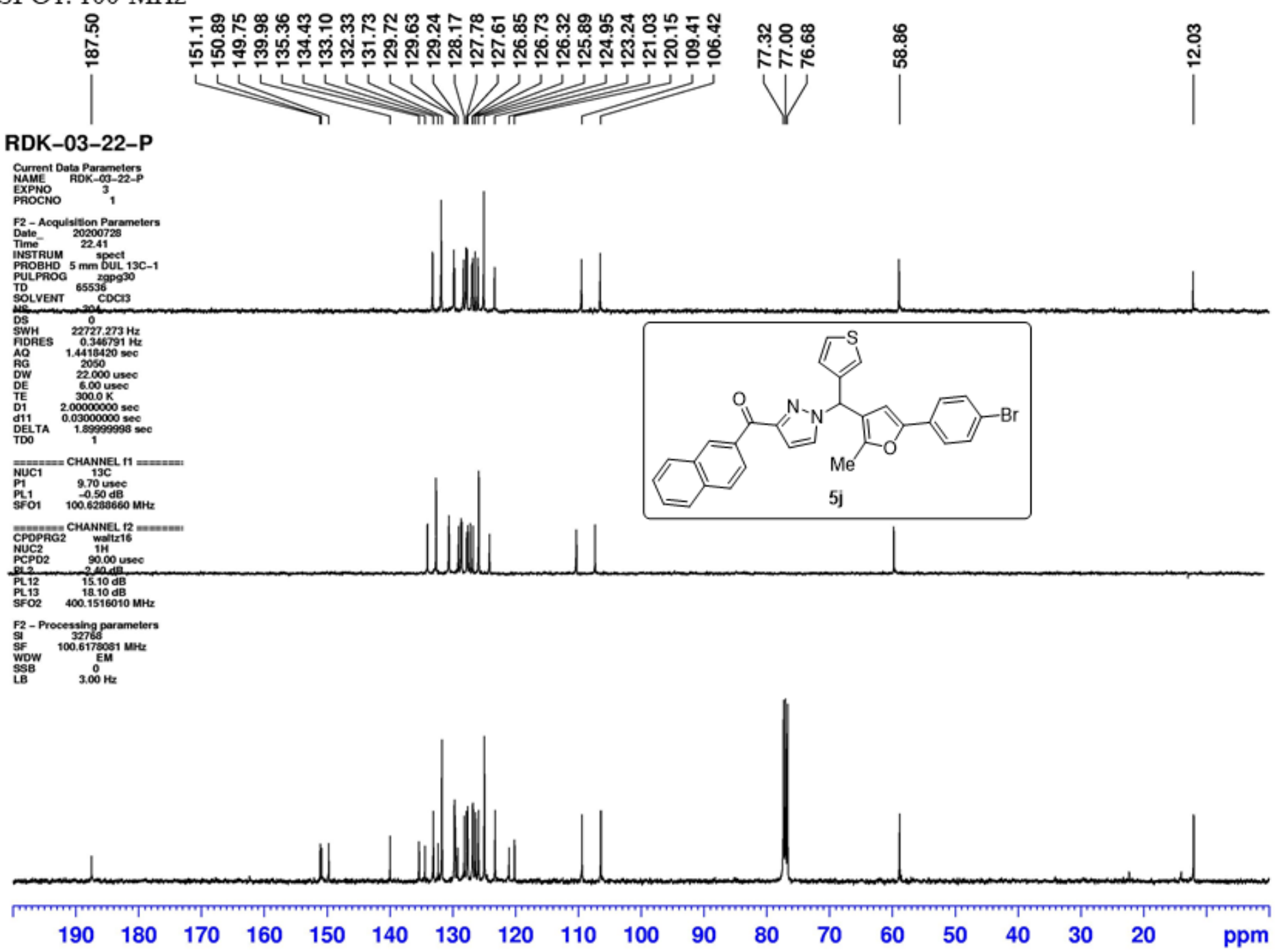


Solvent: $\mathrm{CDCl}_{3}$

SFO1: $400 \mathrm{MHz}$

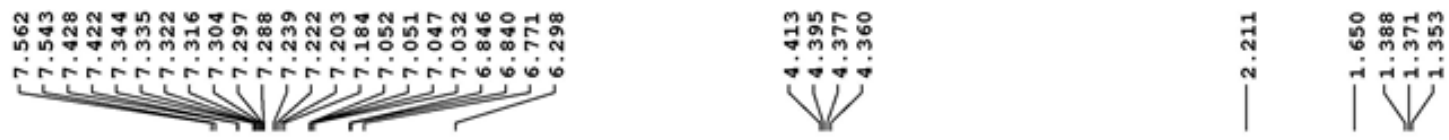

RDK-03-55
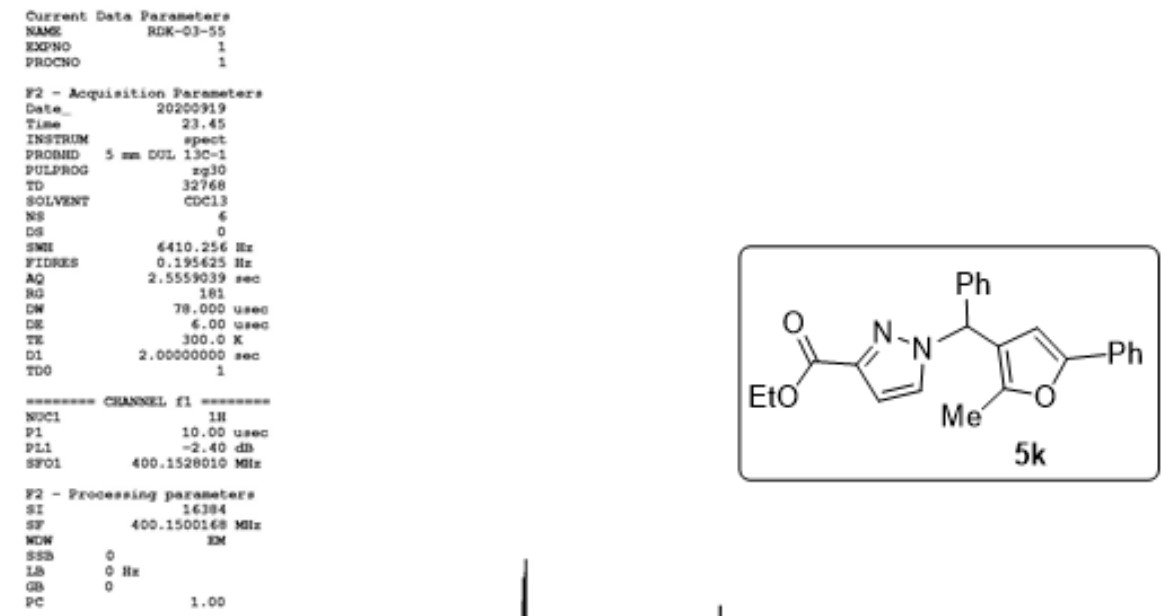

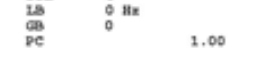

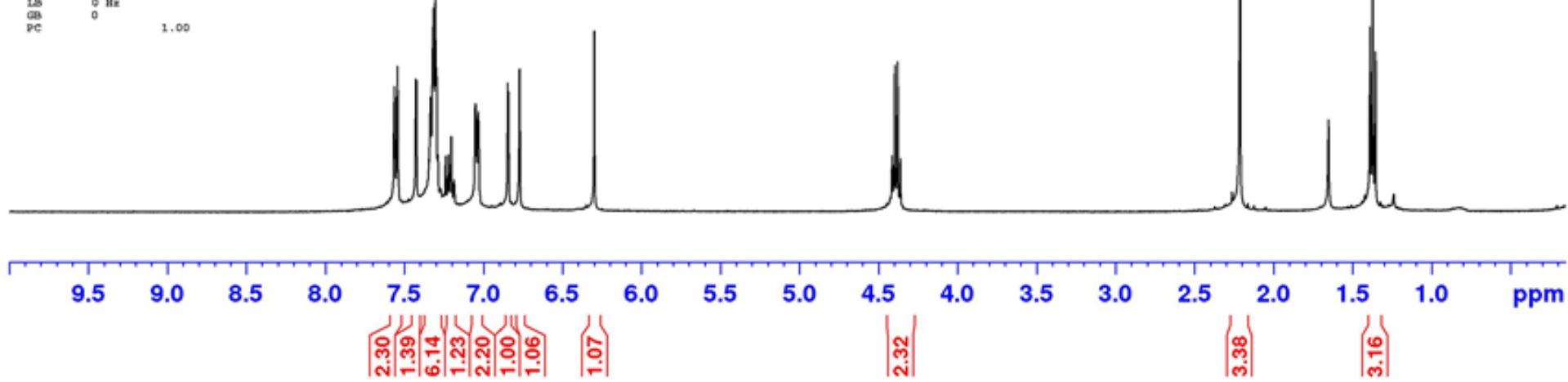


Solvent: $\mathrm{CDCl}_{3}$

SFO1: $100 \mathrm{MHz}$

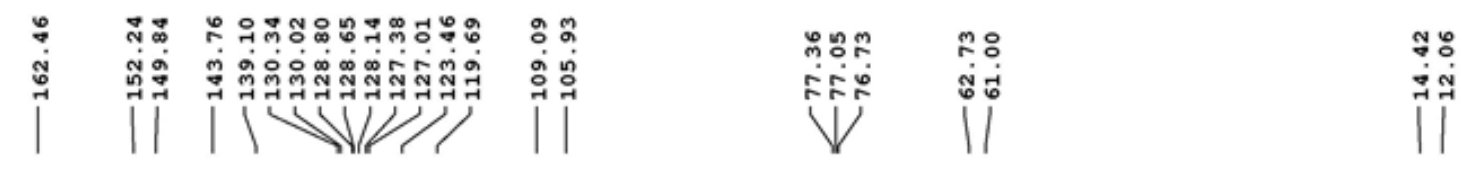

RDK-03-55

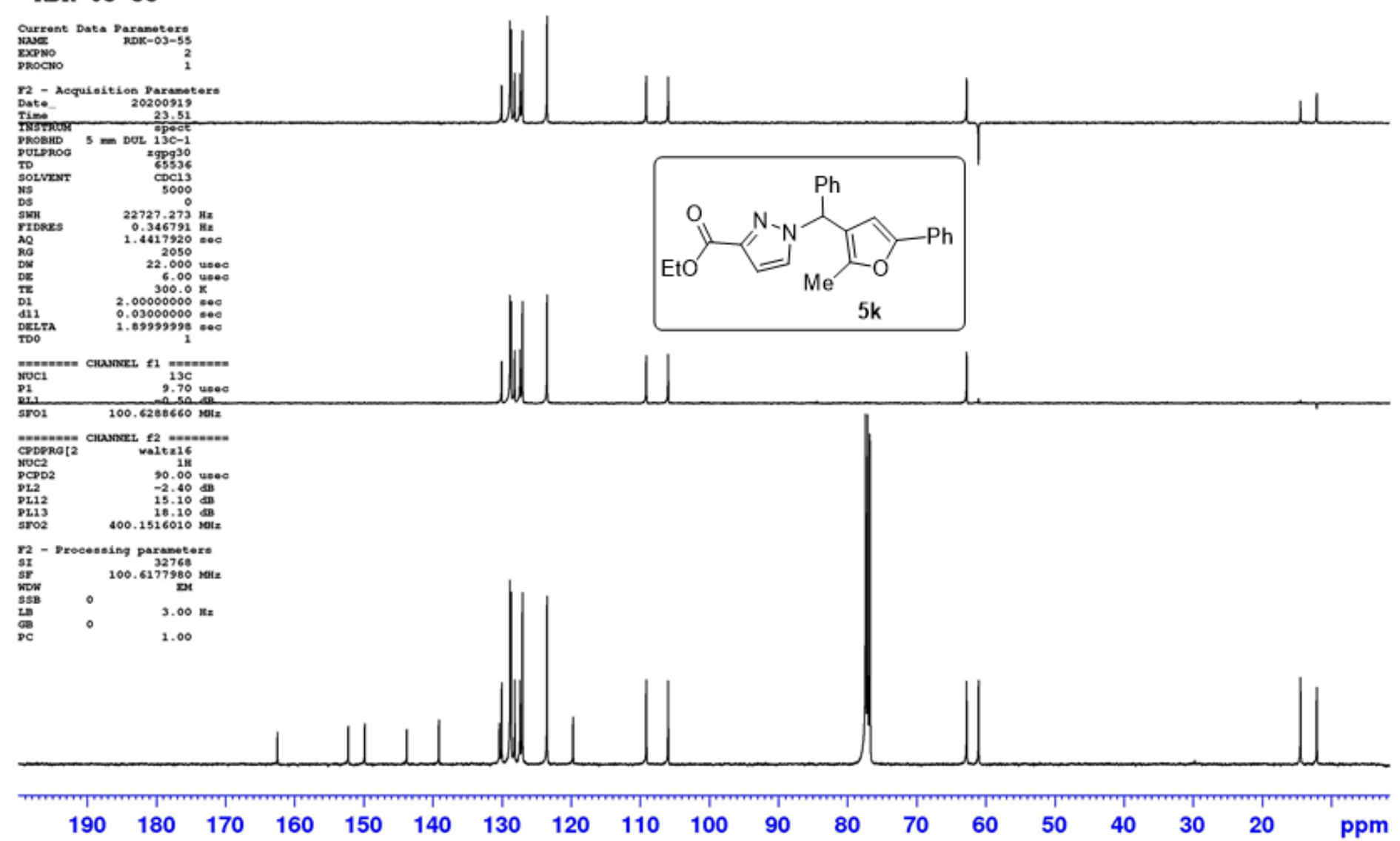


Solvent: $\mathrm{CDCl}_{3}$

SFO1: $600 \mathrm{MHz}$

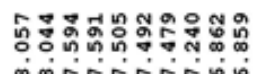

ソं vivivi

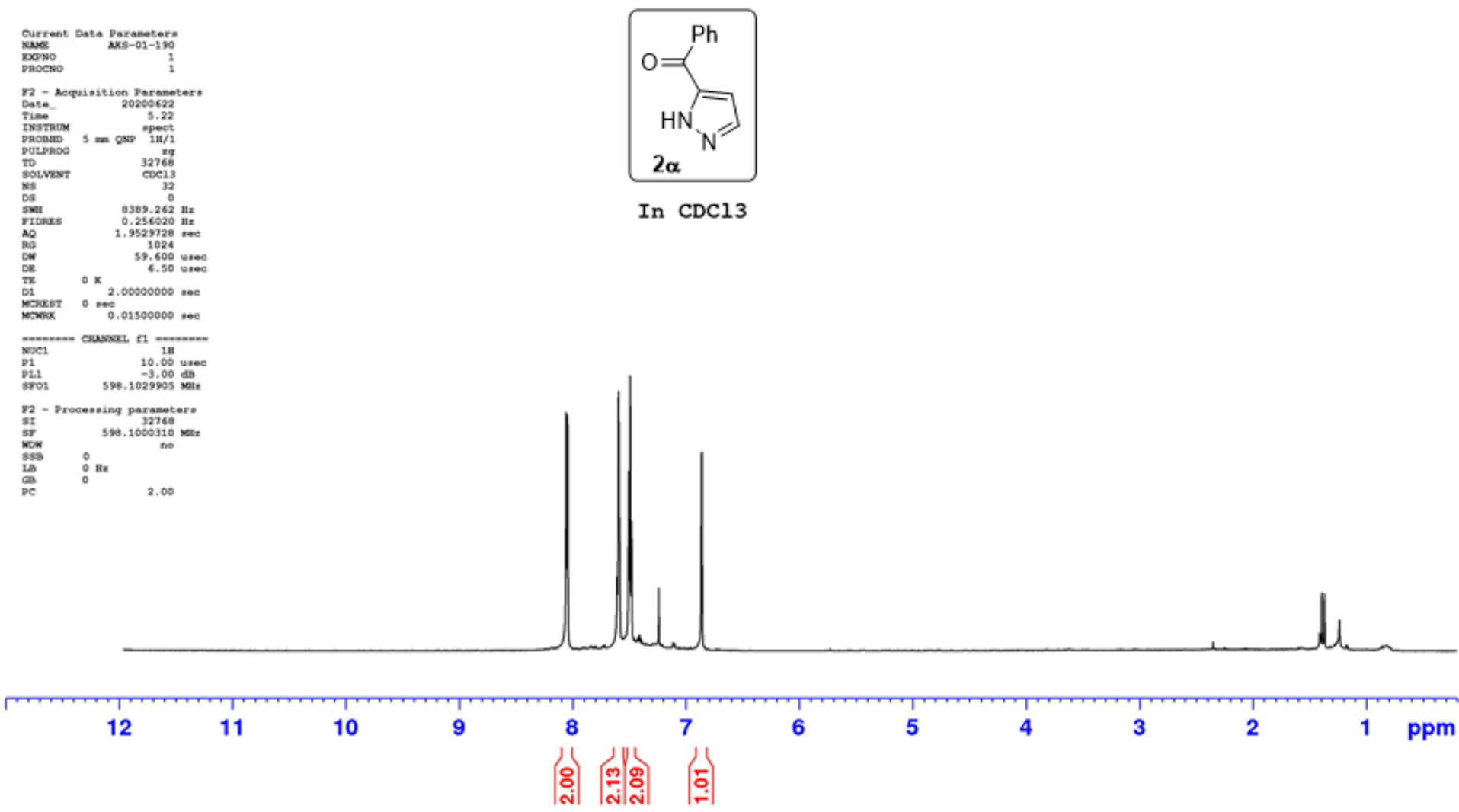


Solvent: $\mathrm{CDCl}_{3}$

SFO1: $150 \mathrm{MHz}$

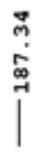

RDK-03-23

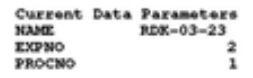

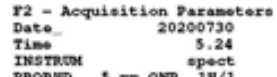

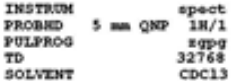

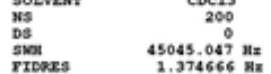

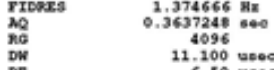

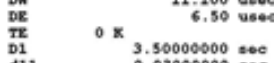

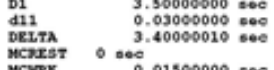

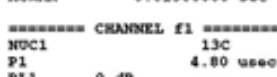

PLi
sro1 ${ }_{150.4096601 \text { satz }}^{\text {dis }}$

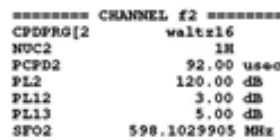

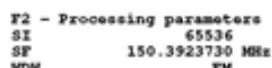

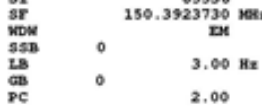

2.00

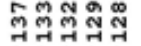

IVII

7)

1

ฟर

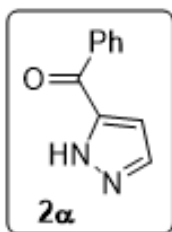

In $\mathrm{CDCl}_{3}$

C.m.

$\begin{array}{llllllllllllllllllll}190 & 180 & 170 & 160 & 150 & 140 & 130 & 120 & 110 & 100 & 90 & 80 & 70 & 60 & 50 & 40 & 30 & 20 & \mathrm{ppm}\end{array}$ 
Solvent: $\mathrm{CD}_{3} \mathrm{CN}$

SFO1: $400 \mathrm{MHz}$

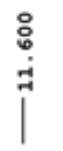

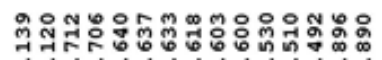

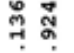

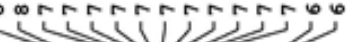

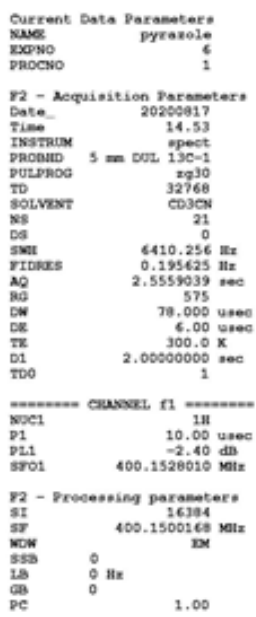

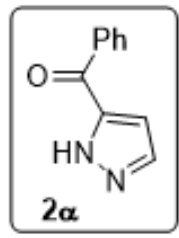

In $\mathrm{CD} 3 \mathrm{CN}$

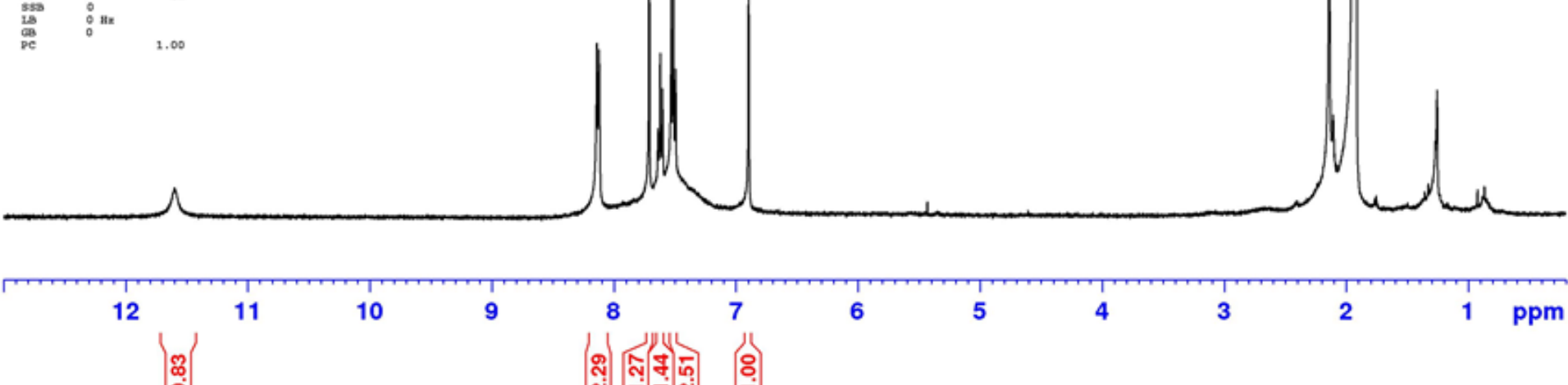

$\left|\begin{array}{c}\dddot{\infty} \\ \vdots\end{array}\right|$

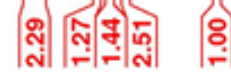


Solvent: $\mathrm{CDCl}_{3}$

SFO1: $400 \mathrm{MHz}$

\section{BRUस्ER}

RDK-02-235-3
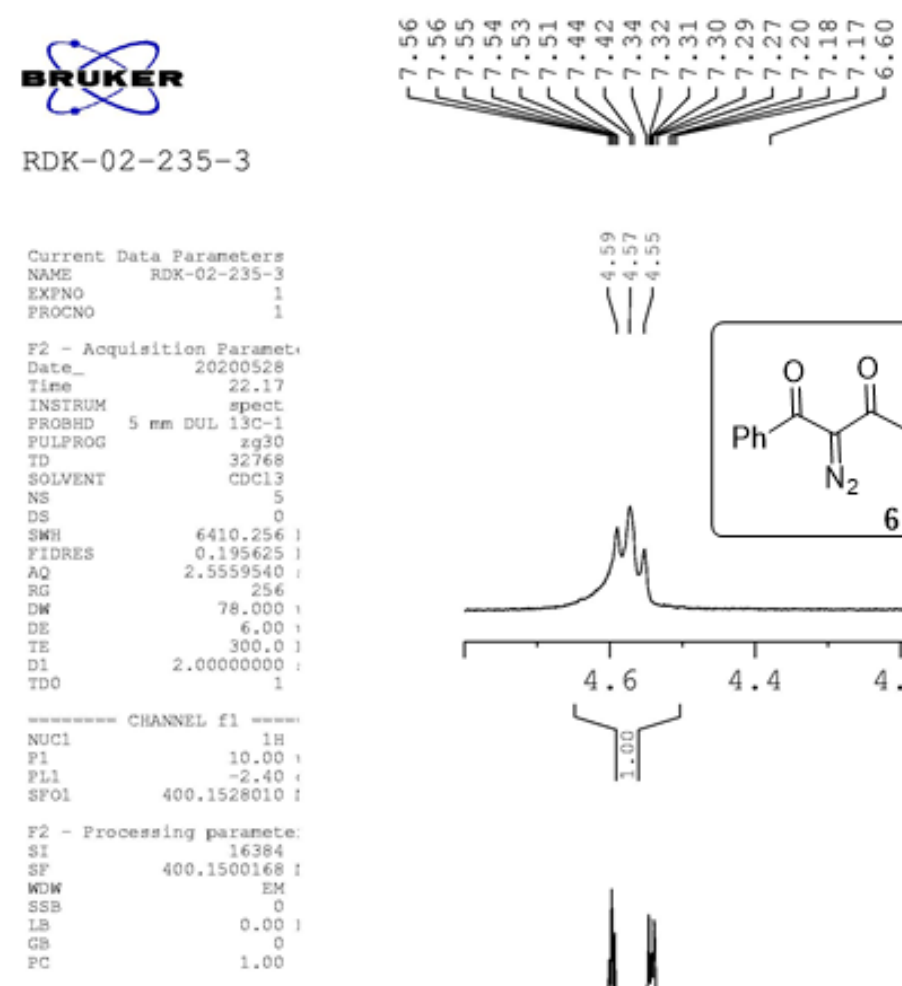

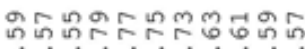

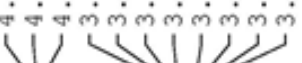
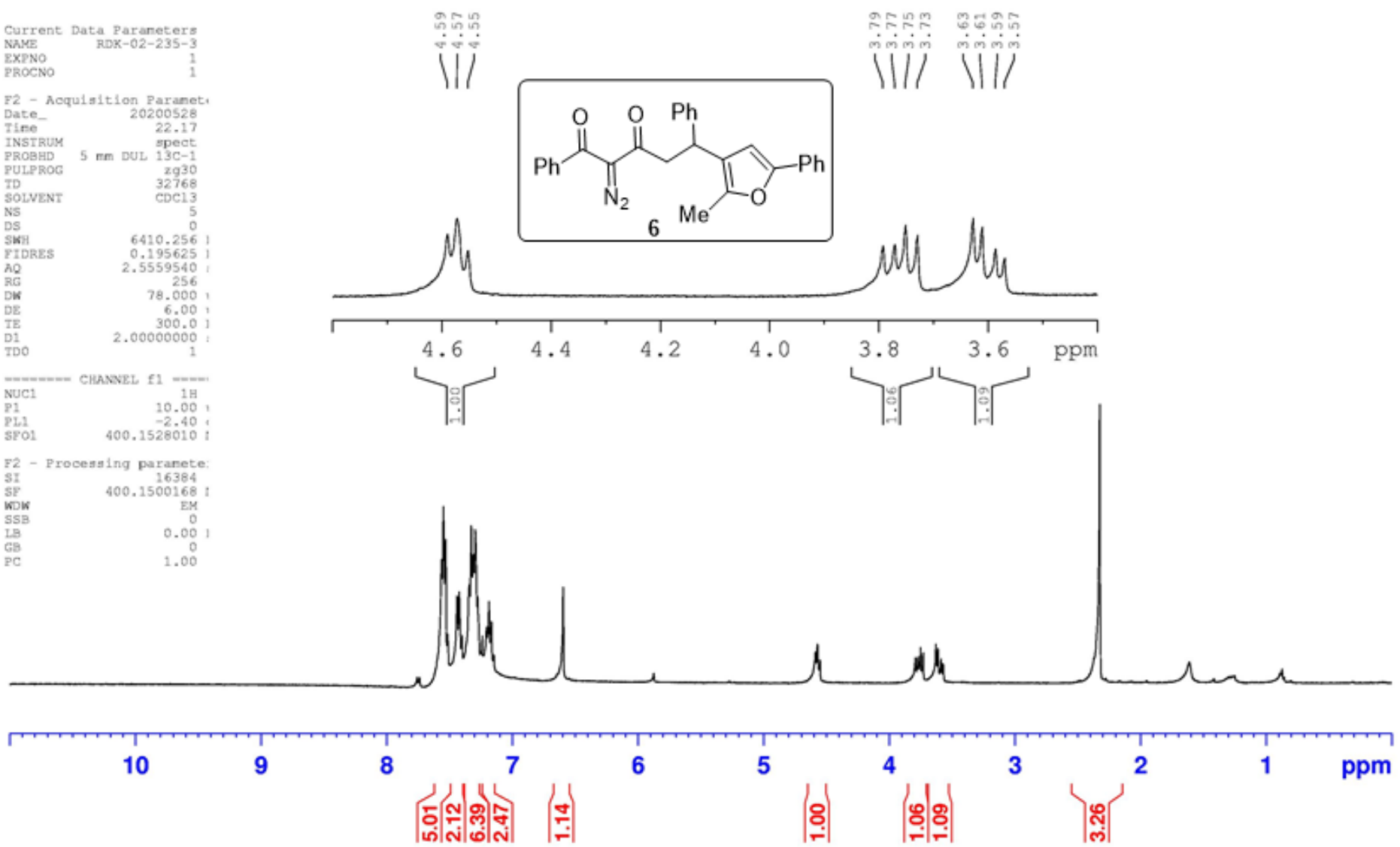
Solvent: $\mathrm{CDCl}_{3}$

SFO1: $100 \mathrm{MHz}$
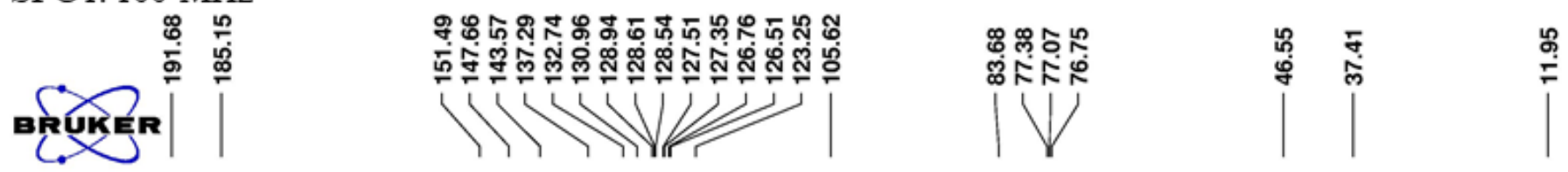

RDK-02-235-3

W

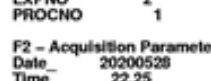

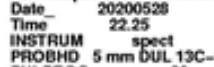

SIDVErT

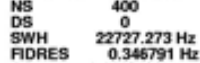

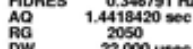

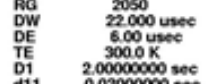

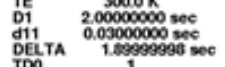

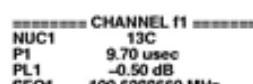

sFO1 $100.6288660 \mathrm{M}$

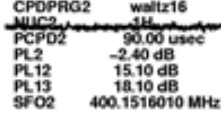

F2 - Procesessing parameters

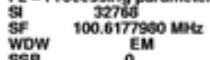

LB $3.00 \mathrm{~Hz}$

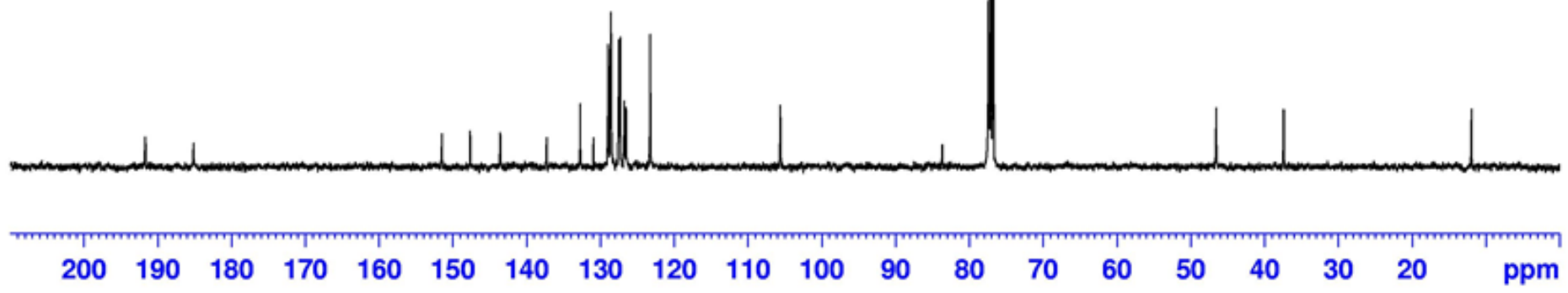

